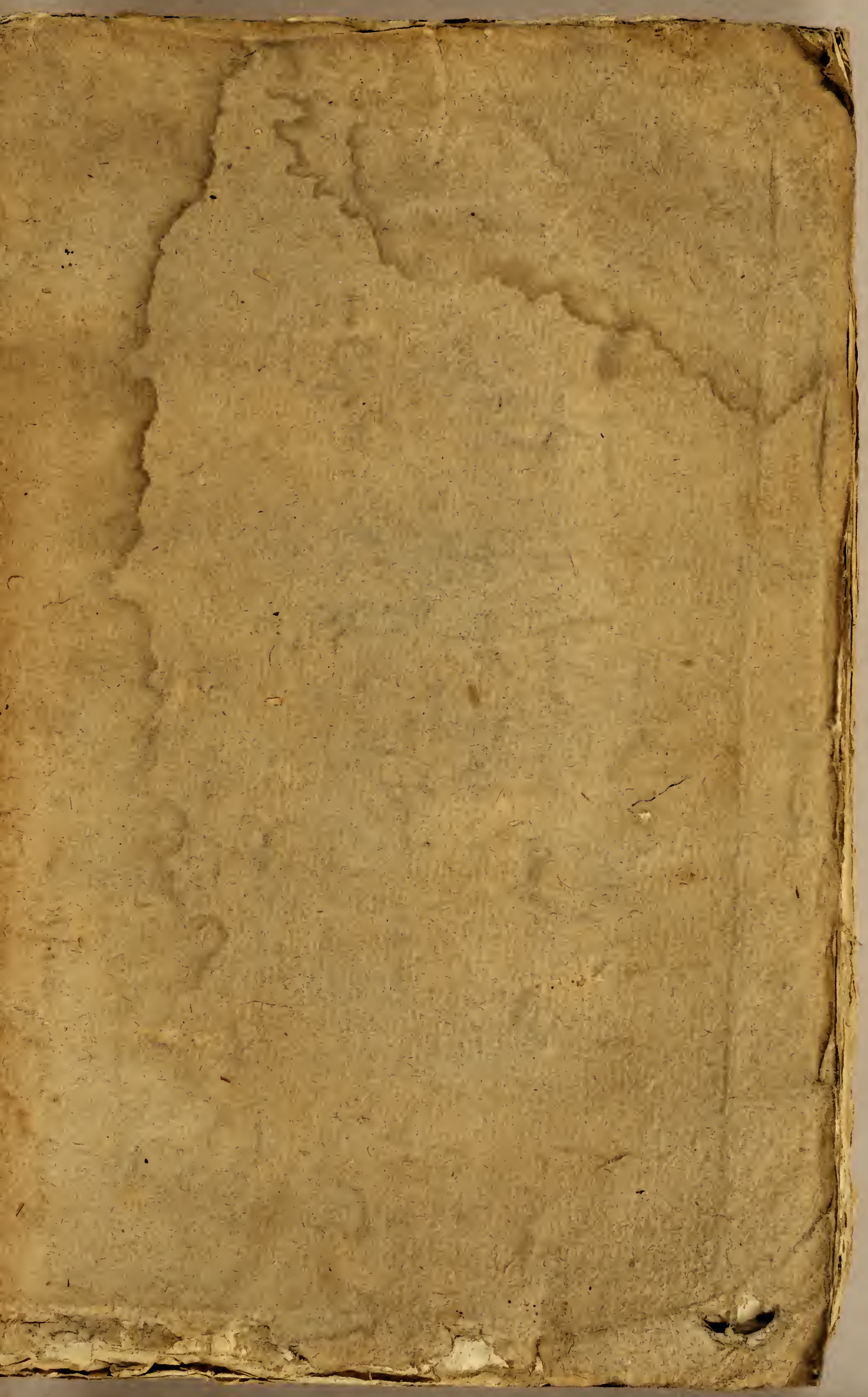




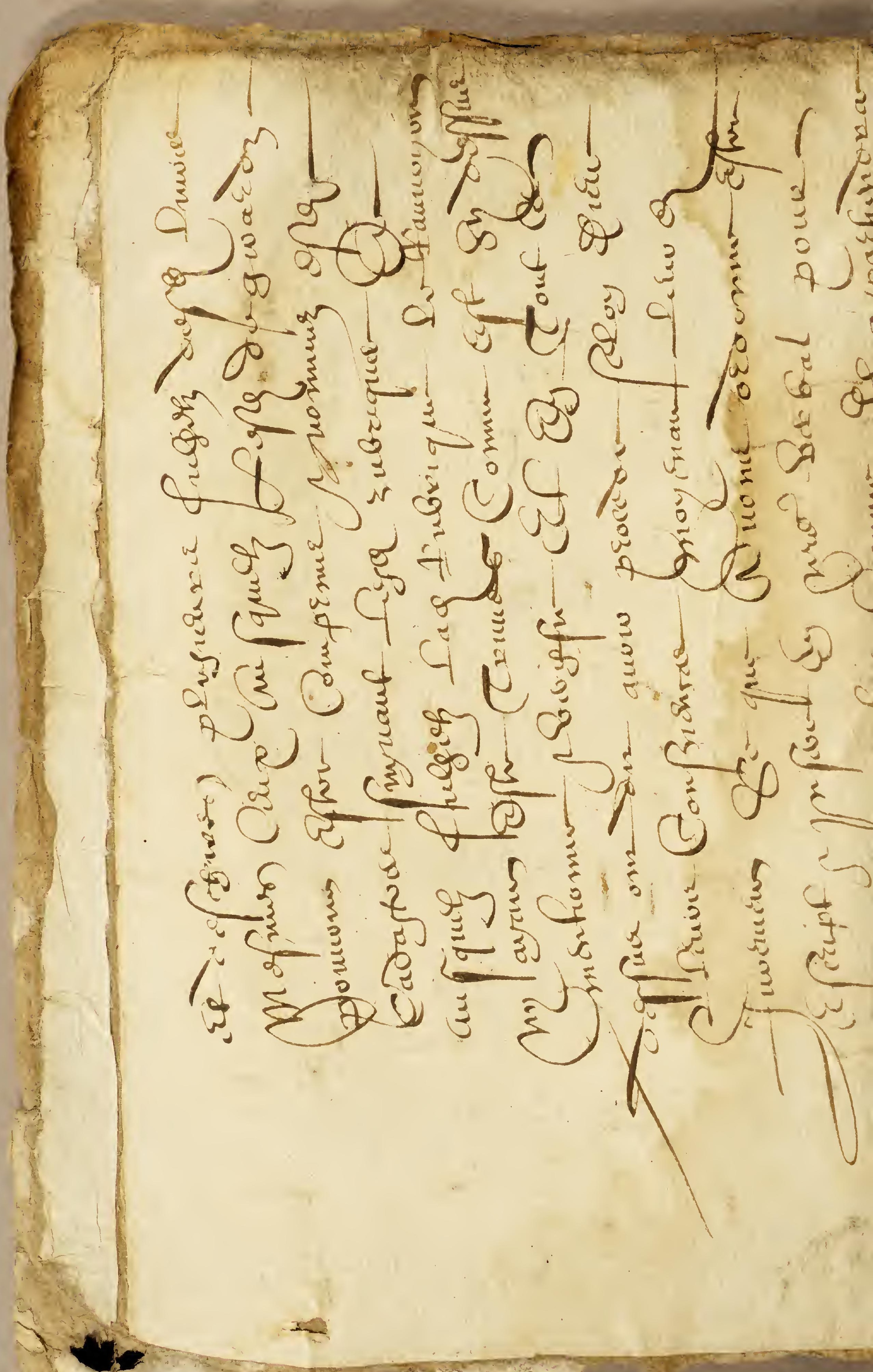


$D E \quad L \cup S A G E$

\section{DU QUINQUINA}

$$
\text { D } A N S
$$

\section{LES FIEVRES REMITTENTES。}


N. B. CE Mémoire fait partie, \& complère le Tome III des Euvres médicinales de l'Auteur.

Le Volume I. contient $I^{\circ}$. un Mémoire fur le vice scrofuleux, couronné par la Société Royale de Médecine de Paris; $2^{\circ}$. un Mémoire fur le carreau, couronné par la Faculté de Médecine de Paris; $3^{\circ}$. un Mémoire fur l'ictère des nouveaux nés, couronné par la méme Faculté.

Le Volume II. contient feulement un traité des convulfions dans l'enfance, couronné par la Faculté de Médecine de Paris, \& par le Cercle des Philadelphes du Cap Français, érigé depuis en Société Royale des Sciences \& Arts.

Le Volume III. contient $1^{\circ}$. un Mémoire fur les effets des émanations marécageufes fur l'économie vivante, couronné par la Société Royale de Médecine de Paris; 20. le Mémoire que nous publions aujourd'hui.

Le Volume IV. contiendra un traité complet fur la phthifie pulmonaire, couronné par la Société Royale de Médecine de Paris. (Cet Ouvrage va être mis fous preffe).

Chaque Mémoire fe vend féparément, \& chaque Volume porte un Titre général, indépendant des Titres particulicrs, en faveur de ceux qui voudront réunir les divers Ouvarges de l'Auteur. 


\section{$D E L U S A G E$ DU QUINQUINA $D A N S$}

\section{LES FIÈVRES REMITTENTES:}

MÉMOIRE qui a remporté, en 1785 , au jugement de la Société Royale de Médecine de Paris, le premier prix sur la question proposée en ces termes:

Déterminer quels sont les avantages E les dangers du Quinquina, adminiftré dans le traitement. des différentes efpèces de fièvres rémititentes.

\section{PAR M. BAUMES,}

Docteur en Médecine de l'Univerfité de Montpellier, agrégé au Collége des Médecins de Nifmes; Médecin de l'Hofpice de Charité le la même Ville; affocié Régnicole de la Société Royale de Médecine de Paris; Affocié National de la Société Royale des Sciences \& Arts du Cap Français; de l'Académie Royale des Sciences, Belles-Lettres \& arts de Dijon, de la Sociéré Royale des Siences de Montpellier, \&c., \&c.

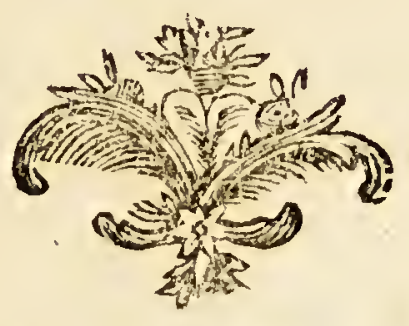

\section{$A \mathbb{P} A \mathbb{R} \mathbb{I}$,} Chez $\left\{\begin{array}{l}\text { Théophile Barrois, Quai des Auguftins, } \\ \text { Mecquignon, rue des Cordeliers, } \\ \text { Croullebois, rue des Mathurins, }\end{array}\right.$

(2)

M. $\mathrm{DCC} \mathrm{XC}=\mathrm{CO}$

Sous le privilége de la Société Royale de Médecine de Paris.

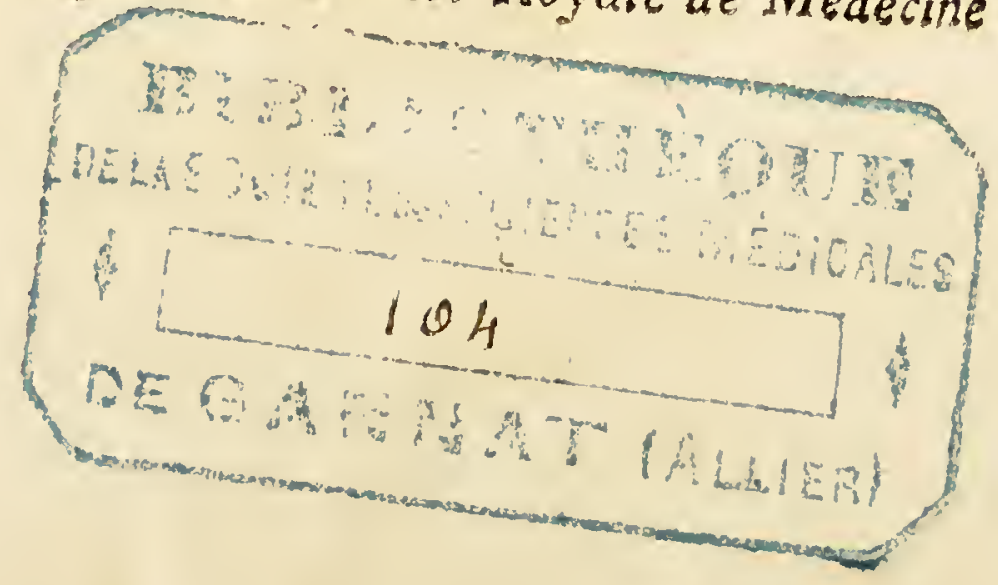


Inepti eft artemque ignorantis, audito mox febris nomine, nullo prorsùs ad materiem febrilem rejpectu habito, largas corticis dofes ingerere.

Stoll, ratio medendi, P. I, pag. 89. 


\section{A L A \\ SOCIÉTÉ ROYALE \\ $D E$ \\ M É D E C I N E \\ $D E$ \\ PAR I S,}

Comme un hommage

qui lui eft bien véritablement dû, pour fes utiles $\mathcal{E}$ honorables travaux;

$\&$

qui lui eft offert, avec refpect;

par l'Auteur :

BAUMES,

'A Jocié Régnicole de la Société Royale de Médecine.

I 790. 



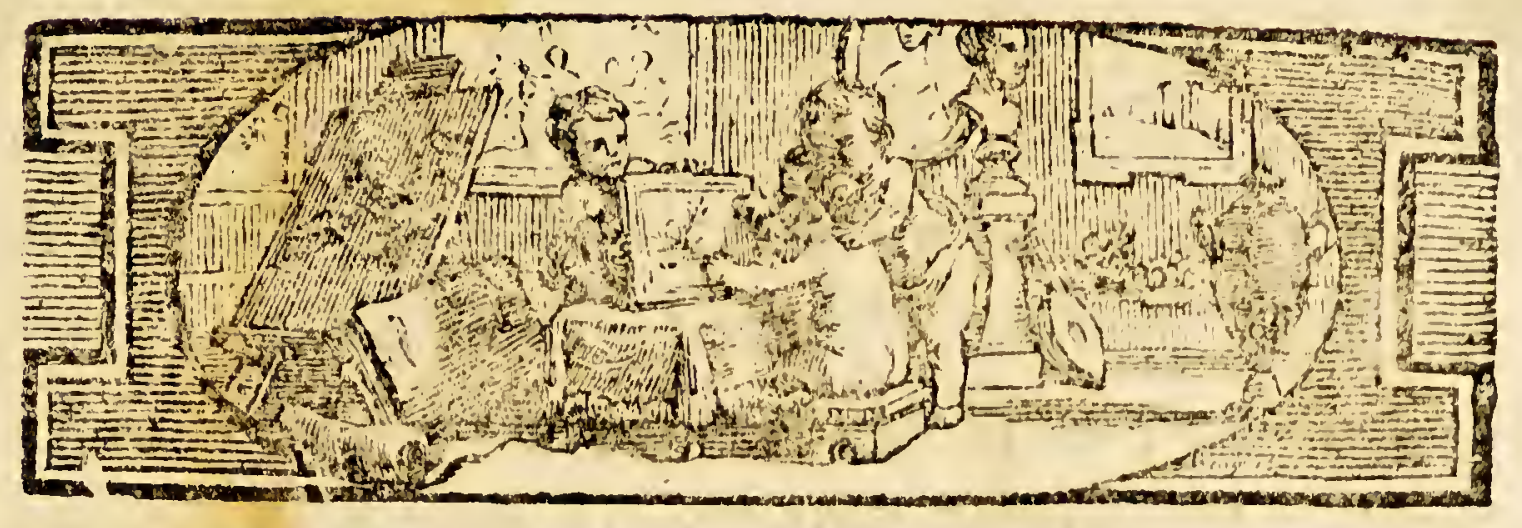

\section{E M O I R E}

$S U R \quad L \cup S A G E$

\section{U Q U I N Q U I N A}

\section{DANS LES FIĖVRES REMITTENTES.}

\$. I, A matière médicale offre peu 4 I de remèdes dont la réputation égale celle du quinquina : cette écorce exotique, une des principales richesses de l'autre hémisphère, qu'une divinité bienfaisante fait végéter au quatrième degré de latitude australe, ainsi que du côté boréal sous une parallèle correspondante. Il est, en effet, peu de miladies qu'on n'ait tenté de détruire avec cette substance, dont les succès ont été d'autant plus marqués, que son application a été plus judicieuse. Mais notre objet est-il de présenter le tableail desinfirmités huma'nes, contre lesquelles le quinquina est un remède plus ou

$\mathrm{A}_{4}$ 
moins assuré ? Devons-nous, par le rapprochement des faits, épurer l'histoire de ce médicament, confirmer ou détruire les propriétés qui Iui sont attribuées, et de l'ensemble de nos recherches, produire un ouvrage lumineux ef solide? Ce travail, au-dessus peut-être de nos forces, n'entre pas dans le plan que nous nous sommes tracés. Qu’il nous suffise ici, pour chercher la solution d'un problème intéressant, donné par la Société Royale, de déterminer quels sont les avantages et les danger's du quinquina dans le traitement des différentes espèces de fièvres rémititenies.

Quelque spécifique que soit le quinquina de la cause matérielle $(\mathfrak{S} . \mathrm{XV}$.) des fièvres rémitzentes, l'observation a démontré que ses bons ou ses manvais effets dépendoient de la légitimité de l'indication. Pour la développer, il faut considérer les fièvres rémittentes sous leurs divers zapports. Il faut fixer leur caractère, et lier en un corps de doctrine les principaux détails qu'on a donnés sur ces maladies,

II. Si riche dans la partie dogmatique, la Médecine ancienne n'offre que des contradictions et des abscurités dans la nomenclature dẹs fières rémitrentes , ce genre de maladie si étendu (I),

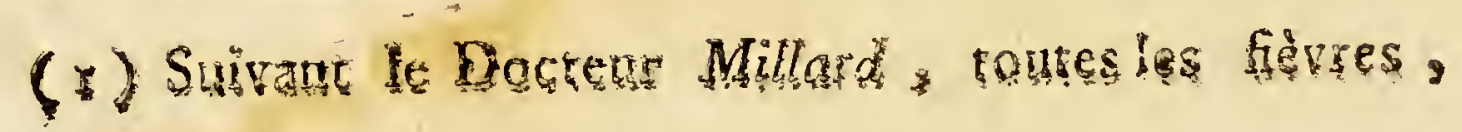


dans les fièyres rémittentes.

si commun dans tous les pays, dans toutes les saisons, sous toutes sortes d'intempéries. Elle ne distingua d'abora' les fièvres qu'en continues et en intermittentes, voulant qu'une marche intense et uniforme, une persévérance égale et vigoureuse, jusqu'd l'entière cessation du mal et la fixité des terminaisons dans une suite de paroxismes, marquassent leurs différences géd. riques. Mais tous les cas ne 'accordant point avec cet arrangement systématique, elle se contenta ensuize de restreindre ou d'étendre les significations d'intermitrence et de continuité: voulant qu'une fiève fut principalement et uniquement dans la classe des continues, lorsquelle ne changeoit point de nature jusqu'à son jugement critique, et qu'une fièvre ne sortît pas du genre intermittent, quand même elle ne cesseroit point à la terminaison de la fièvre, ou qu'elle souffiroit des changemens remarquables en chaque accès, soit dans le temps du commencement, soit dans celui de l'augmentation, de la vigueur ou du déclin.

excepté les éphemères \& les fièvres inflammatoires, font du genre des rémittentes, \& ne diffèrent entr'elles que par le degré d'intenfité. M. Lieututud a de même avancé que la fière continente, qui, felon l'idée des Anciens, n'a ni rémifion, ni exacerbation, n'exifte à la rigueur gue dans les lives. 
10 De l'usage du Quinquina

Ainsi les fièvres réellement rémittentes étoient assimilées à raison d'une analogie dans la marche, aux fièvres foncièrement intermittentes. Une nouvelle classification les rapporta au genre continu, et ce genre fut divisé en fièvres synoques et fièvres sync̀ques: les synoques comprenarit les maladies oì l'ardeur de la fièvre n'a aucune intermission, et la dénomination de synèque étant affectée à toutes celles qui, n'ayant pas d'apyrexie réelle, ont cependant deux périodes trèsmanifestes, l'un de recrudescence ou de vigueur de la fièvre, l'autre de rémission ou de trèsgrande réduction respective de tous les mouvemens fébriles.

Cette division n'étoit point équivoque; mais les Latins qui crurent faire mieux en donnant aux noms grecs, ceux de leur idiome, rendirent synoque (I) par continente, et synèque par continue, et renversèrent ainsi la vraie étymologie du mot, ou du moins le sens qu'il falloit donner à la chose. Alissi, frappé par cette

(I) C'eft par une fuite de l'anarchie qui règne dans la divifion des fièvres, que Eller a claffé, parmi les fynoques, les continues rémittentes; que Waldschmitt dit que les fynoques de fon climat ne font pas communément continentes, mais continues rémittentes, \& que M. Grant appelle fynoque non putride, la véritable fièvre rémittente du printemps. 
dans les fièvres rémittentes.

contradiction, Morton voulut appeler continues, les fièvres qui le sont réellement, c'esi-ḋ-dire, celles qui n'éprouvent aucun changement considérable et dépendant de la nature de la fièvre, et assigna le terme de continentes, aux fievres qui ne font pas paroître une exacte régularité jusquà leur terminaison, à celles dont l'ardeur de la fièvre a des exacerbations et des rémissions, c'est-d̀-dire, qu'elle est sujette à diminuer dans un temps et à redoubler dans un autre; en un mot, à celles dont les changemens périodiques sont de l'essence même de la maladie.

Qui croiroit que la vanité des Nomenclateurs eût eu quelque chose de plus à désirer? Ce fut elle qui fit appeler paroxismales, par Avicenne; continues périodiques, par Sennert; proportionnées, par Torti; rémittentes, par Huxam et Pringle; continuées par quelques-uns; exacerbantes ou fièvres avec redoublement, par les $\mathrm{Pa}$ thologistes : les maladies aiguës, appelées génériquement continentes, par Morton; continues, par les Scholastiques; Synèques, par lécole d'Hippocrate. Est-ce à raison de cette discordance, que le plus grand nombre des Auteurs, faisant des genres particiliers de certains symptômes qui ne font qu'exprimer différens états de la fièvre (I),

(1) M. Clarck confidérant la diverfité qui règne dans les 
12 De lusage du Quinquina

ont préféré la dénomination de putride, de bilieuse, pour traiter véritablement des fièvres rémittentes?

III. Heureusement que ces titres arbitraires ne changent rien au fond de l'objet, et qu'on est à peu près d'accord pour appeler rémittentes la fièvre, qui, sans cesser un moment depuis le commencement jusqu'àla fin, est divisée

fièvres, \& blâmant la trop grande multitude des claffes qu'on a établies nous dit que, malgré fon application à étudier, pendant plufieurs années, les fymptômes \& la nature des fièves en divers climats, il n'a jamais pu reconnoître ce grand nombre de différentes fièvres que les Auteurs admettent ; \& il eft convaincu que quoiqu'il y air des variétés qui tiennent à la conftitution \& au climat, il n'en eft pas moins vrai que par-tout la fière eft effentiellement la même; qu'il n'y en a qu'un feul genre, qui ne comprend que trois efpèces; favoir, la fièvre intermittente, la rémittente \& la continue. La divifion qui établit des fièvres inflammatoires, des fièvres nerveufes, des fièvres putrides, eft vicieufe, parce qu'en examinant de près ces genres fuppofés, on voit qu'ils ne font qu'exprimer différens états de la fièvre, \& que les fymptômes qui les caractérifent, accompagnent également les fièvres intermittentes, les rémittentes \& $x$ les continues; ainfi, quelqu'attention que ces états de fières méritent dans la pratique, comme ils fe rencontrent également dans les fièvres intermittentes, rémittentes $\&$ continues, on ne doit pas les regarder comıne des abjets d'une différence générique. 
dans les fièyres rémittentes.

par des périodes très-courts, et qui se succèdent régulièrement; chaque période étant composé d'un temps d'orage, pendant lequel le malade agité, souffrant, est dans un état, dont les symptômes nouveaux qui se présentent, augmentent assez rapidement jusqu’à un certain point, et décroissent ensuite d'une manière presqu'aussi subite, pour disparoitre en tout ou en partie: c'est ce qu'on appelle exacerbation, redoublement, paroxisme ou reprise; et d'un temps de calme, pendant lequel le malade, beatscoup mieux, éprouve une diminution sensible dans les accidens, et reste ainsi dans un état fébrile jusqu'à l'invasion d'un autre paroxisme: c'est ce qu'on appelle rémission; de sorte que pour avoir une définition exacte de la fièvre rémittente, il faut la tirer de sa marche aiguë et soutenue, que coupe une série plus ou moins périodique d'exacerbations ressemblantes, quoique distinctes et formées par des symptômes, qui, dans leur courte durée, émanent de la nature même de la maladie, et ne correspondent point à des causes manifestes.

La fièvre rémittente paroît donc être une fièvre d'une espèce particulière, faisánt la nuance entre la fièvre continue et la fièvre intermittente. Comparée avec la fièvre continue, elle peut parôitre intermittente, et comparée réciproquue- 


\section{\$4 De l'usage du Quinquina}

ment avec la fière intermittente, elle peut paroître continue. Aussi, n'est-il point étonnant que les anciens Médecins, qui souscrivoient pour appeler continues rémittentes, les maladies marquées par une régulière alternative de paroxismes et de rémissions, l'apyrexie n'étant jamais parfaite, les qualifiassent tantôt simplement de continues, et d'autres fois d'intermittentes, suivant que les fièvres qu'ils décrivoient, s'étoient plus rapprochées des unes que des autres; et que les modernes qui ont vu tant de rapports entre les fières intermittentes et les rémittentes, aient regardé ces deux fièvres comme formant deux ordres très-voisins l'un de l'autre, au point même que quelques-uns les ont confondues et n'en ont fait qu'une seule classe. Nous nous expliquerons ailleurs $(\$ . X V$.$) sur cette analogie.$

IV. Nous le ferons encore remarquer. Pour qu'une fièvre soit réellement de l'ordre rémittent, il ne suffit pas qu'elle ait des augmentations et des diminutions relatives des mouvemens fébriles; il faut encore que la succession des paroxismes et des rémissions soit à pen près (I)

(1) Nous difons à peu près, parce que, dans plufieurs cas de fièvres rémittentes, foit bénignes, foit malignes, les paroxifmes dévancent d'environ deux heures; mais comme cet avanccment eft régulier, notre propofition n'êt pas démentie. 
dans les fièvres rémittentes. périodique; il faut que l'invasion du paroxisme arrive sans cause étrangère, et que la rémission soit spontanée (I). Sans ces conditions essentielles, toutes les fièvres seroient rémittentes, parce qu'il n'y en a presque point qui soient véritablement homotones. Il n'y a point de fièvre qui ne varie, quant à son intensité; il n'y en a point dans laquelle les médicamens un peu forts, même les boissons et les alimens qu'on donne aux malades, ne leur occasionnent des variations remarquables dans le mouvement fébrile. On sait d'ailleurs que, dans les fièvres du type le plus continu, les symptômes augmentent d'intensité tous les soirs, comme le pouls chez les personnes saines : phénomène constant qu'on doit moins attribuer à la nature de la fièvre, qu'à l'action de l'air, qui, pendant ce temps, accélère, dans tous les individus, le mouvement du pouls d'une manière plus ou moins sensible. Ces raisons et quelques autres

(I) Dans une fièvre continue, mille circonftances, telles que le bouillon, le mouvement, \&c., procurent des chaleurs pafiagères, connues fous le nom de bouffées, comme d'autres circonftances, telles que les évacuations procurées par les purgatifs, par les faignées, font des rémiffions ; auffi Lautter demande-t-il, pour foupçonner une fièvre rémittente, qu'il y ait une grande rémiffion fans caufe aflignable. 
semblables, obligent en conséquence de cires conscrire la dénomination de rémittentes, aux fièvres dont le caractère est bien marqué par une alternative de reprises et de rémissions, dépendante de la nature même de la fièvre. Et pour trouver une marque plus distinctive en. core de ce genre rémittent, oubliant le rapport que les reprises ont entr'elles, eu égard à leur totalité, à leur durée, ou aux symptômes qui caractérisent leurs différens temps, parce que ces temps se confondent dans quelques circonstances, nous la prendrons cette marque plus distinctive dans lobservation de la rapidité de la marche du paroxisme. Ainsi, quelle que soit une fièvre rémittente, c'est toujours avec une espèce de mouvement accéléré, que la maladie s'avance vers son plus haut degré, et s'en éloigne ensuite. De sorte qu'une exacerbation de fièvre rémittente, sera toujours reconnoissable, en combinant le changement de l'état du malade de bien en mal, et de mal en bien, avec la briéveté du temps, dans lequel ce changement s'est opéré. C'est une idée que nous devons à MM. Voullone et Senac.

Tels sont les indices que nous croyons caractéristiques des fièvres rémittentes. Un paroxisme et une rémission en constituent le période, et ce période suit en général la marche que nous allons décrire. 
dans les fièrres rémittentes.

V. Le paroxisme se prépare, et la révolution qui doit le produire, s'annonce, dit moins poutr lordinaire, par des signes qui dénotent la dé pravation des sucs, l'embarras des entrailles, tels qu'un sommeil lourd, fatigant ou interompu, une bouche sale au réveil, une douleur ou simm plement une pesanteur de tête, des yeux pesans et rouges ou abattus, de la tristesse, du dégont, des nausées, une chaleur incommode qui se firt sentir principalement à la paume des mains, ê à la plante des pieds, etc. L'orage est près du terme où il doit éclater, et les phénomènes quí l'indiquent sont ceux qui caractérisent la foiblesse, le spasme, la congestion du sang dans les parties internes, un refoulement général de la circonfém rence vers le centre. On se plaint de malaise et d'un sentiment de forte lassitude, qui est̃ caușể par la tension spasmodique de toute l'habirude du corps, et notamment du système musculaires: tension que le malade tâche de dininuer en se repliant en divers sens, et fléchissant soutes les articulations, pour relâcher, le plus qu'il es. possible, les parties tendues. Dans ce même temps, il y a une envie de dormir presquinin surmontable; les ongles sont pâles et livides le bout du nez et l'extrémité des doighis sont froids; la respiration est gênée, et particulière* ment l'inspiration est pénible a aussi les bâillemẹta 
et les pandiculations (I) deviennent-ils fréquens. Le malade eșt encore pénétré par intervalles d'une sensation extérieure de froid, et ressent des douleurs obtuses, qui ne viennent que de la tension des muscles. Son pouls est d'abord lent, il devient ensuite fréquent, mais il reste encore quelque temps petit, et ne se développe que par la succession des révolutions organiques. Le pouls n'est d'abord lent qu'à cause de la surcharge du sang dans le cour et les gros vaisseaux; mais le sang agit sur le coeur comme un stimulus, et y excite des contractions plus fréquentes et plus promptes.

Le spasme parvient au comble, et le tremblement universel en est le signal. Le frisson commence par les parties les plus éloignées du cœur. Il se propage peu à peu jusqu'à ce quỉil ait gagné les parties internes, et le malade éprouve alors pendant quelque temps des bouffées alternatives de froid aigu (2) et de chaud

(1) Tont le monde connoît la théorie du bâillement qui n'eft qu'une donble infpiration. Quant à celle des pandiculations ; en élevant le bras, on fixe l'attache des mufeles grand pectoral \& grand dorfal; \& par ce moyen, ils peuvent, dans leurs contractions, élever les côtes avec plus de facilité, et aider l'infpiration.

(2) Sans donte que ce fentiment de froid tient beaucoup à la force du fpafme, \& d'autres caures, que cs 
obscur ; pendant le froid, il tremble, et ses mâchoires se heurtent; pendant le chaud obscur, il est assoupi et dans un sous-délire. D'ailleurs il est pâle et défguré; il a une petite toux sèche, une soif assez forte, accompagnée d'une bouche sèche, pâteuse ou amere, et des envies fréquentes d'uriner; en outre, la respiration est plus ou moins gênée; il y a des náusées, le pouls est petit, fréquent et serré, la tête fait mal ; les sujets très-irritables et très-nerveux, éprouvent des mouvemens convulsifs; quelques-uns tombent dans le délire; les urines tenues et pâles, sont quelquefois troubles; et cette variété vient du plus ou du moins de spasme dont sont affectées les voies urinaires.

Tant que dure le premier temps du paroxisme ou celui du froid, les malades sont très-impatiens, parce qu'ils sont perpétuellement dans le spasme, et que la nature lutte péniblement contre des causes d'une destruction imminente; et cet état dure plus ou moins, selon la violence de la maladie, la disposition du sujet et quelques autres circonstances particulières. Midis il diminue insensiblement; les frissons, les tremblemens et les mouvemens convulsifs, deviennent

n'est pas ici le lieu d'expliquer; mais pour en rendre raifon, on n'a pas affez confidéré jufqu'à quel point $y$ coopéroit la faculté frigorifique du principe de la viéa

B 2 
plus rares; les secousses que produit le froid intérieur, s'éloignent de plus en plus. On remarque alors dans le pouls des alternatives de concentration et de développement; de petites bouffées de chaleur coupent par intervalles le sentiment de froid; les vomissemens surviennent ou sont plus abondans, s'ils ont déjà eu lieu; quelques malades tombent en syncope. Le froid cesse, une chaleur douce et générale lui succède, et le pouls se relève. Plus de nausées, de vomissemens, d'ischurie, du moins c'est l'ordinaire; car il n'est pas si rare qu'on le pense, que le frisson cesse, sans que les symptômes propres à cet état se dissipent aussitôt; de sorte que les malades, quoique dans le chand, ont des nausées, des vomissemens, la respiration courte et gênée, le pouls petit et serré pendant quelque temps.

Affaissé sous le poids des couvertures, le malade demande d'en être délivré; il se découvre, cherche le froid. Son visage s'anime et rougit; l'haleine est brûlante, les yeux sont étincelans; $1^{a}$ peau est très-sèche et chaude; la soif devient plus urgente; la respiration, quoique moins gênée, est plus fréquente; le pouls est plein, fort, égal et précipité; le battement des artères, mais sur. tout celui des temporales et des carotides, se fait sentir vivement; la tête fait mal, et quelquefois 
dans les fièvres rémittentes.

le délire et même le transport se mettent de la partie. Les douleurs du dos, des lombes, des articulations, les maux de tête, tous les symptômes, en un mot, vont, pendant un certain temps, en augmentant, ês soutiennent ensuite dans un état de balancement; les malades s'aperçoivent bientot des alternatives de diminution et de retour à cet état d'équilibre. Mais les accidens déclinent, tombent; la peau est tour-à-tour chaude, sèche et brûlante, ou chaude, tempérée et humide; enfin, elle s'assouplit peu d̀ peu et s'humecte; les urines qui avoient été difficiles ou même arrêtées, commencent à couler aisément et en abondance; de rouges et enflammées, elles deviennent épaisses et sédimenteuses; dans cerrains cas, le sédiment ressemble à de la brique pilée; la moiteur se déclare, et le paroxisme qui avoit d'abord présenté les indices d'une maladie grave, finit ou par la sueur, ou par des selles bilieuses, quelquefois par l'une et l'autre de ces évacuations.

Les malades se trouvent assez bien après ce paroxisme. Il y en a même qui, pendant la rémission, se trouvent assez dispos, pour croire qu'ils n'ont essuyé qu'un accès de fièvre intermittente; cependant le pouls reste sensiblement fébrile; la bouche est sale, ou l'haleine fétide; $1^{\text {a }}$ peau n'est point douce, et sa chaleur n'est 
22 De l'usage du Quinquina

point naturelle; le regard n'est pas net, et tous se plagnent de brisemens, d'anorexie; ils ont une disposition habituelle au frisson, etc. Le second paroxisme revient avec plus de violence; le premier temps étant en géréral moins marqué; et on en peut dire autant de toutes les autres exacerbation, dont l'intensité paroit augmenter d'une reprise à l'autre, jusqu'à ce que la fièvre soit parvenue à son état, pour décroître ensuite presque dans les mêmes proportions, que les paroxismes avoient augmentés, le premier temps devenant de nouveau plus lucide.

VI. Ce tableau du période d'une fièvre rémittente, ne diffère que par des nuances, par quelques épiphénomènes de plus ou de moins, lorsque la maladie est bénigne. Mais l'addition de la malignité, présente ce période sous une autre face. Car, une maladie n'est véritablement maligne, que lorsque le système général des forces du principe vital se trouve affoibli par une vraie résolution des forces de tous les organes, qu'ont produites les causes primitives de cette maladie en portant le plus grand désordre dans la succession des fonctions (I); aussi, dès le commencement de ces fièvres, les forces

(1) Voy. M. Barthez, nouv. élém. de la fcience de lhornme, tom. s, pag. 255 . 
dans les fièvres rémittentes.

animales sont ordinairement abattues, de même que les forces vitales; le pouls est habituellement mou et foible, presque toujours petit et enfoncé, souvent inégal; les accidens qui s'y développent, ne répondent pas toujours au degré de la fièvre. Les nausées, les vomissemens opiniâtres ( $\mathbf{r}$ ), avec cardialgie et anxiété, le cours de ventre séreux, bilieux très-liquide, le gonflement du visage, la surdité, l'assoupissement, les douleurs profondes et cruelles, le délire, plutôt sourd que furieux, l'affection léthargique, la dificulté de respirer, le météorisme

(I) On donneroit, felon nous, une idée faufe ou limitée de la malignité, fi, pour défigner fon caractère pathogrioinonique, on ne la faifoit réfider dans les affections fortes \& durables du principe vital. affections exprimées, ou par une foule d'épiphénomènes très-graves, qui dérangent prodigieurement, \& d'une manière dangereufe, la marche de la maladie, ou par l'abattement des forces \& la foibleffe du pouls, qui ne permettent pas de compter fur les refources de la nature. Nous difons durables, c'eft-à-dire, qu'il faut une perfévérance marquée dans l'un de ces deux états, parce qu'il y a dans les fièves une infinité d'affections paffagères très-alarmantes, qui fe dilipant aifément, ne doivent pas être mifes au rang des accidens qui conftituent les fièvres malignes. (C'eft ce que j'ai établi dans un Mémoire Jur les fievres malignes, qui a remporté l'accedit au jugement de 'Académie de Dijon, Eq que je publierai bientót). 
34 De liusage du Quinquina

du bas ventre, lincertitude des mouvemens, légarement des sensations, st en général, le trou bie de toures les foncrions dépendantes du système nerveux, surviennent très-ordinairement, quoique le pouls demeure petit, enfoncé, mou, foible; il est cependant quelques épidémies où le mouvement du pouls est assez violent.

A ces signes non équivoqués, on ne méconfoît point la malignité d'une fièvre rémittente. La maladie n'est point déclarée, que les malades: affrissés, tristes, mornes, se plaignent de vertizes, de menaces de défaillance et de tous les symprômes, portés à un plus haut degré, qui précèdent le paroxisme d'une fièvre bénigne. Hexacerbation commence, et les malades, qui: sont pris tout à coup d'un froid considérable dans toure l'habitude du corps, avec des dou. leurs plus ou moins grandes, qui semblent para ir de l'épine du dos, ont un pouls presquoblicéré, qui ne se fait remarquer que par des ribrations confuses, par un frémissement accéléré qui émeut à peine le tact: les sujets immobiles et gelés se plaignent de spasmes, de pesanteum, de traillement dans les premières voies: to vomissenent qui sannonce bientôt est le 1 his sonvent de la bile verte, etc. A lintensine do ce froid, succede nne pive chaleur, beancoup. dagiation le pous s'étere latament lors 
dans les fièvres rémittentes.

même qu'il est le plis développé, il est plus ou moins foible, mou, petit, inégal, à moins que l'influence du tempérament ou de l'épi 'émie ne rende le pouls plein, haut, accélíré. inégal dans le rithme et dans la force des vibri... tions, ou dur et tendu. Tous ont des symptô. mes proportionnés à cet état d'accablemont ce de perversité : symptônes qui varient d'après le caractère épidémique - :ur peau dure et sèche, brûlée d'une ardeur âcre et mordicante beaucoup plus forte que dans les fières continues, n'entre en sueur que douze ou quinze heures après. Cette exacerbation terminée, les malades sont abattus, languissans, avec une impression de chaleur et de feu dans la bouche; leur pouls est concentré, petit, inégal, guère plus vîte que dans l'état de santé; et quelquefois ce pouls ressemble parfaitement, ou même est plus rare que le pouls naturel; les malades rendent des matières séreuses, vertes, bilieuses, fétides, etc.

Le nouveau paroxisme se manifeste avec plus de violence; le froid en est plus profond, plus durable, et les autres symptômes sont à proportion. Les épiphénomenes s'accumulent, s'aggravent, et à la quatrième, cinquième ou sixième exacerbation, la rémittence est déjà obscure, ou le type continu: tuneste dégéné- 
26

\section{De l'usage du Quinquina}

ration annoncée sur-tout par la limpidité des urines, de troubles qu'elles étoient auparavant, et par la disparition de leur sédiment terreux qu'on avoit trouvé sur la fin des premières reprises.

VII. Comme on le pense bien, tous les symptômés énoncés dans le diagnostic général $(\mathbb{S}$. V et VI. ) d'un période de fièvre rémittente; ne sont ni constans, ni essentiels. Ceux qui le sont et qui appartiennent foncièrement au paroxisme, peuvent être réduits dans les trois temps qui le divisent; $I^{\circ}$ a au froid, au frisson, ou à un état spasmodique violent, pendant lesquels le pouls est petit, concentré et fréquent, pour ce qui concerne le premier temps; $2^{\circ}$. à la chaleur, à l'agitation dont le développement du pouls est inséparable, pour ce qui regarde le second ; $3^{\circ}$ a a r relâchement général qui amène la rémission de la fièvre et de ses accidens avec quelques excrétions critiques, pour ce qui est du dernier. Faut-il encore, pour que cette marche naturelle ait lieu, que la maladie ne soit point anomale, illégitime? Et combien de fièvres dont le type est obscur' et la marche irrégulière, dans lesquelles le premier temps est très-court ou foiblement prononcé, ou même absolument insensible, tandis qu'on n'aperçoit qu'une diminution momentanée des 
accidens du troisième, que le retour du prenier absorbe en quelque sorte. Une pareille confusion, propre seulement aux fièvres trèsgraves et à exacerbations subintrantes, nous fait sentir plus que jamais, combien le diagnostic seroit difficile à saisir, si l'on oublioit les indices caractéristiques que nous avons assignés ( $\$$. III et IV.) aux fièvres rémittentes : caractères évidens, et qu'on trouve également dans les maladies équivoques comme dans les plus compliquées.

VIII. Quant à la rémission, quoiqu'elle se fasse reconnoître à la diminution et à la chûte des symptômes propres aux paroxismes, on pourroit quelquefois la confondre avec l'intermission, si, pour prononcer, on ne vouioit s'appuyer que sur le rithme dú pouls. Mais, c'est moins quelquefois sa fréquence qui constitue l'état fébrile, que la collection de plusieurs symptômes qui indiquent la lésion des fonctions. En effet, il est de toute notoriété que ceux qui ne veulent juger de l'existence de la fièvre que par la fréquence du pouls, sont exposés à confondre, avec les intermittentes, plusieurs cas de fièvres rémittentes malignes, pendant la rémission desquelles le pouls est naturel, ou même plus rare que celui-ci: rithme, qui même quelquefois, au lieu de paroître pendant la rémission, 
ne se fait apercevoir qu'au plus haut degrẻ du paroxisme. Mais quels sont les praticiens qui s'en laissent imposer par ces indices de malignité? Ils savent que la gravité des. simptômes ne s'estime pas en raison de lappareil menaçant ou de la manière orageuse avec laquelle une maladie s'annonce; au contraire, que plus il y a d'abattement, de foiblesse du côté des forces vitales, comparées aux forces musculaires, plus les symptômes sont graves, dangereux et malins; et qu'au défaut des indices fournis par la fréquence du pouls, on a, pour juger de l'existence de la fièvre, la prostration des forces, les anxiétés, une lésion notable dans les organes de la tête et les précours, la gêne de la respiration, une diminution des sécrétions marquée par la soif, la sécheresse de toute l'habitude du corps, les urines rares et rouges, etc.

IX. Si ces assertions ( $\$$. VIII) sont fondées, on se voit obligé de restreindre considérablement la classe des fièvres intermittentes malignes, pour augmenter à leurs dépens celle des fièvres rémittentes. En observant avec attention ces sortes de fièvres intermittentes malignês, on trouve effectivement qu'elles n'ont aucun intervalle lucide, dans lequel la force du pouls, respectivement à la force des membres, soit la même que dans l'état de santé, ou, si le pouls est 
le même, quant à la fréquence, que celui d'un sujet sain, la foiblesse des membres est plus grande que dans les intervalles des vraies fièvres intermittentes. Telle étoit l'opinion de Sauvages : aussi ce Professeur célèbre renvoyoit-il les fièvres malignes qui ont l'apparence des intermittentes, aux fièvres putrides, malignes, aux tierces, aux quartes continues, c'est-à-dire, aux vraies fièvres rémittentes. Mais Hippocrate est le père de cette doctrine. On sait que cet illustre Observateur a déclaré absolument exemptes de péril, toute espèce de fièvre intermittente.

X. Quoi qu'il en soit, nous n'irons pas plus loin sans remarquer que la lucidité du période, ( $5 . \mathrm{V}$ ) formant une marque générique d'une fièvre rémittente; que la lucidité des trois temps $(\mathbb{S}$. VII ) qui constituent une exacerbation, exprimant la marche naturelle de la maladie; enfin, que la lucidité di premier de ces temps, annonçant la légitimité du type rémittent, c'est d'après ces principes qu'on peut établir trois ordres de fièvres rémittentes. Le premier comprend toutes les fièvres dont chaque paroxisme débute par le frisson; le second renferme toutes celles dont les reprises commencent ou par un refroidissement de tout le corps, ou par un simple refroidissement des extrémités et du nez, ou par une toux sèche et plus ou moins vive; le troi- 
sième rassemble toutes celles dont les exacerbations riont, dans leurs premiers temps, ni frisson ni froid, ni refroidissement partiel, et ne sont remarquables que par la recrudescence de la fievre, par une augmentation de chaleur âcre et des autres accidens fébriles qui décroissent, après être montés à leur plus haut période; observant toute fois que la première oules deux premières exacerbations des fievres comprises dans le second $t \boldsymbol{t}$ le troisième ordre, commencent par un frisson qui est ordinairement considérable; et que si le premier temps des autres redoublemens est en général insensible ou peu marqué, le dernier temps est bien lucide : car, tant que la fièvre n'est pas dénaturée, la sueur ou des urines très-chargées terminent toutes les reprises. Les fièves du premier ordre sont les plus légitimes; et dans le fait, on pourroit, en quelque sorte, les considérer comme de véritables intermittentes, que leurs accès prolongés font paroître continues. Celles du second ordre le sont beaucoup moins, et semblent constituer les vraies rémittentes; mais celles du troisième sont obscures, illégitimes, et rentrent, pour ainsi dire, dans la classe des fièvres du type continu. Cependant, comme dans les fièrres de ces deux ordres, le premier ou les premiers paroxismes débutent par le frisson, et que la fin des reprises est forte- 
dans les fièvres rémittentes.

ment prononcée, leur droit à la famille des vraies rémittentes doit être conservé, plus sans doute en vertu de leur périodicité, qu'ì cause du précepte de Galien, qui dit que les fievres qui s'annoncent par des frissons, doivent être comptées au nombre de celles qui sont sujettes à des retours réguliers.

XI. Tant que les symptômes qui se développent dans les exacerbations, se bornent à une augmentation de la fièvre et des incommodités qui ont coutume de l'accompagner, telles que le mal de tête, les inquiétudes, la chaleur, la soif, peu de sommeil, la fréquence de la respiration, et que le pouls se maintient développé, la fièvre rémittente est d'une nature simple et bénigne; elle l'est moins lorsque le paroxisme amène du délire, un peu d'oppression, une toux incommode, un météorisme modéré; mais sa nature est grave lorsqu'il survient dans les reprises, soit des foiblesses, des syncopes; soit un délire frénétique, ou une affection soporeuse, apoplectique, ou des mouvemens convulsifs, ou un météorisme excessif, ou les symptômes de la pleuresie, de la péripneumonie, de l'hépatite, de la dyssenterie, d'une inflammation de basventre, etc., et que le pouls devient petit, mou, inégal: signe affecté au plus grand nombre des fièvres graves ou malignes. 


\section{2}

De l'usage du Quinquina

XII. On ne peut se méprendre sur la valeur des symptômes de la pleurésie, de l'hépatite, etc. ( $\$$. XI. ) Ils sont un produit de la fièvre rémittente, parce qu'ils lui sont évidemment subordonnés; la fièvre s'est d'abord manifestée sans eux; ils n'ont paru qu'au second, au troisième paroxisme, et même plus tard; ils ne laissent aucune trace on du moins qu'une trace très-obscure, pendant la rémission, pour sévir avec autant de vigueur et même plus d'intensité dans l'exacerbation suivante: la fièvre est donc'alors véritablement rémittente, et peut seulement recevoir l'épithete de pleurétique; d'hépatique, comme pour désigner ceux des accidens qui sont les plus remarquables. Au contraire, si les symptômes énoncés étoient prédominants dès le commencement de la maladie, c'est-à-dire, qu'ils parussent avant ou en même temps que la fièvre, et continuassent avec vigueur pendant la rémission, ils constitueroient une maladie compliquée, dans laquelle la fièvre rémittente seroit subordonnée, ou tout au plus marcheroit de pair avec la pleurésie, l'hépatite, etc. Dans le premier cas, la fic̀vre est l'accident principal, et tout au plus. les épiphénomènes tendent à prendre le dessus; dans le second, c'est la maladie qui mérite la première considération, et la fièvre peut tendre à dominer sur elle : distinction importante et qu'it 
dans les fièvres rémittentes.

ne faut jamais perdre de vue. Malheur aux malades, si, par une funeste erreur, on prend les symptômes pour la maladie essentielle, et les reprises, pour les recrudescences d'une fièvre continue.

On ne s'attend pas sans doure que nous présentions ici le tableau des fièvres rémittentes péripneumoniques, hépatiques, soporeuses, etc. Ce travail seroit d'autant plus superflu, qu'en réunissant les signes $(\$ . V$ et VII ) de la fièvre rémittente, et ceux de la maladie qui en est un produit, on aura le diagnostic de la fièvre rémit. tente dont il s'agit. Consignons cependant ici les principaux traits d'une fièvre rémittente pleuropéripneumonique, qui fut la fièvre épidémique de la constitution pendant l'automne de 1782 , tant parce que ces fièvres étant plus communes, il convient de leur consacrer quelques détails, que pour donner un exemple qui nous dispense de produire des tableaux de fièvre hépatique, soporeuse, dyssentérique, etc.

XIII. Depuis plusieurs années, les fièvres intermittentes et rémittentes sont et plus communes dans ce pays, et beaucoup plus fâcheuses. Elles forment l'épidémie la plus générale et la plus longue qu'on ait observé depuis bien dú temps. En 1780, sette constitution ( $\$ . \mathrm{XLI}$.) 
s'étant renforcée, les malades furent très-nombreux; et ayant reçu quelques modifications en I782, l'épidémie changea de face, et ne nous présenta plus qu'une fièvre rémittente pleuropéripneumonique.

Les malades offroient les avant-coureurs d'une fièvre putride et bilieuse; et après trois, quatre, six jours d'incubation, plis ou moins, ils étoient attaqués de la première exacerbation, semblable en tout à un paroxisme ordinaire $(\mathrm{S} . \mathrm{V} \cdot)$ de fièvre rémittente, qui ne se termineroit que par une crise très-incomplette. Ici les fébricitans ne suoient que de la poitrine, du cou, de la tête, le reste du corps étant sec et chaud. Pendant cette première rémission, la respiration étoit plus gênée et plus fréquente que de coutume; la langue se couvroit d'une crasse jaunâtre; les déjections étoient fétides, bilieuses; la peau conservoit une chaleur âcre, etc.; et le second jour étoit occupé par une autre reprise beaucoup plus foible que celle du jour précédent; son premier temps étoit obscur, et la respiration moins libre, après qu'elle étoit terminée. A l'exacerbation du troisieme jour, aussi forte que celle du premier, le frisson très-bien marqué étoit court et suivi d'une toux sèche et urgente; la poitrine faisoit mal, et ordinairement il s'établissoit un point 
dans les fièyres rémittentes.

douloureux ; l'oppressión augmentoit, étoit considérable pendant la chaleur, et le maläde, crachoit une humeur séreuse et sarguinolente; quelquefois le sang sortoit presque pur, d’autres fois il étoit confondu dans une espèce de gêlée d'un blanc jaunâtre. Il n'étoit plus question de ces accidens pendant la rémission, et les indices de cacochylie putride-bilieuse (\$.XXXI. XXXII.) dominoient à leur tour. La quatrième reprise, correspondant à la seconde, étoit exempte des symptômes de la pleuropéripneumonie, à moins que la maladie ne fût d'une fâcheuse espèce. Dans ce cas, cette quatrième exacerbation devenoit aussi considérable que la troisième; et si l'on ne $s^{\prime} y$ opposoit pas, ou si les remèdes étoient infructueux, le produit de la fièvre dominoit bien. tôt sur elle. Dans les cas ordinaires et les plus communs, les symptômes pleuropéripneumoniques qui se déclaroient à la troisième, à la cinquième ou à la septième exacerbation, c'est-ìdire, à peu près dans l'état de la fièvre, n'avoient lieu que dans les grands paroxismes des jours impairs. Mais si la maladie étoit mal traitée, elle suivoit la marche propre à celle qui, de sa nature, étoit très-grave; et quioique les accidens de la pleuropéripneumonie devinssent dominans, if se faisoit a chaque reprise une nouvelle congestion d'humeurs dansle poumon, qui causoit bien-

$\mathrm{C}_{2}$ 
tôt des lésions irremédiables et mortelles. La mort n'étoit retardée ou prévenue que par une abondante expectoration dans la rémission suivante. A mesure que la maladie prenoit une mauvaise tournure, la langue se séchoit et noircissoit; les exacerbations n'étoient remarquables que par la recrudescence des symptômes de $\mathbf{l a}$ pleuropéripneumonie et de la fièvre; le ventre se météorisoit; il survenoit une diarrhée trèsputricie, et les malades mouroient avec cette multitude d'épiphénomènes qui accompagnent ordinairement une maladie mortelle.

Telle fut la marche de notre épidémie. Sarcone (I), qui a vu ce genre de maladie, rapporte l'histoire d'une fièvre presque semblable, dans laquelle le caractère rémittent étoit très-bien marqué, au commencement de la maladie, par un frissonnement qui annonçoit fort clairement les reprises; mais lorsque les paroxismes devenoient plus confus, relativement à leur invasion, on n'observoit plus ce frissonnement qui reparoissoit ensuite sur le déclin de la maladie, lorsque le type devenoit plus distinct. Un accident remarquable de cette épidémie, c'est qu’à chaque exacerbation, le poumon se chargeoit d'un amas d'humeurs qui devoient être expectorées dans la rémission suivante. Quelquefois, dans l'état de la

(1) Iftoria ragionata de mali ofervati in Napoli, \&c. 
dans les fièzres rémittentes.

maladie, les poumons étoient si affoiblis, si engorgés par la congestion réitérée de ce viscère, que le malade couroit les" plus grands risques, si les remèdes n'agissoient très-vite.

XIV. Jusqu'ici nous n'avons examiné notre sujet, que d'une manière plus ou moins générale. Un ouvrage tel que le nôtre admet-il d'autre plan? Cependant nous devons faire plus : nous devons, dans nos détails ultérieurs, consiciérer les fièvres rémittentes sous un aspect plus particulier; et pour tout embrasser, nous parlerons d'abord de leurs causes, du type qu'elles affectent, du génie qui les distingue, enfin des complications qui les dénaturent.

$X V$. Et d'abord, quant à leurs causes, avec le désir și naturel et peut-être bien pardonnabie à l'homme, de deviner le secret de la nature dans la production des causes premières des maladies, on ne tarda pas à s'apercevoir qu'il existe deux espèces de miasmes ( $I)$, ayant chacun des qualités et des effets propres, différens les uns des autres; les premiers constituant une classe de miasmes éminemment contagieux, soit par leur nature extrêmement subtile, soit par la disposition qu'ils acquièrent dans le corps de l'animal,

( I) Voyez Mémoires fur les fièvres \& fur la contagion, traduits du Docteur Lind, par M. Fouquet, pag. 252.

$\mathrm{C}_{3}$ 
émanent du corps des hommes ou des animaux (principalement lorsqu'ils sont attaqués de maladies fiévreuses:, ou de leurs cadavres. Leur nature nous est inconnue; et ce n'est que par conjecture qu'on pourroit la faire consister dans le gaz hydrogène ( air inflammable) très-pur, combiné avec une portion d'ammoniaque (alkali volatil), et d'huile très-subtile et pénétrante: produits constans des substances animales, livrées aul mouvement de putréfaction qui les décompose; et leurs résultats, lorsque ces miasmes affectent les corps vivans, sont d'occasionner des fievres putrides malignes, d'un type foncièrement continu. Les histoires épidémiques donnent la solution et la preuve de cet énoncé; et il n'est pas de notre objet de nous y arrêter davantage.

Les miasmes de la séconde espèce sont ceux que la chaleur du soleil, dans les climats chauds, élève, en certaines saisons, des lacs ou étangs, des marais ou terrains marécageux, et antres (1)

(I) Par les expériences faites avec l'air nitreux, pour marquer les différens degrés de falubrité de l'air qu'on refpire, il eft démontré que le plus falubre n'eft point altéré par un fol de rochers, de pierre, de fabie, de craye, d'argille; \&x que fur un fleuve, fur une rivière \& même fur la mer, loin du rivage, il eft dans un haut degré de falubrité pendant les vents du nord- 
dans les fièvres rémittentes.

dont la surface est comme dissoute et pétrie par des pluies ou des inondations. On. peut ranger encore dans cette classe quelques exhalaisons nuisibles de la terre. Leur nature ne nous est pas

oueft \& ouest, \& pendant les pluies à groffes ondées. La terre graffe végétale l'altère très-foiblement. la fange des marais, quand elle eft très-fèche, ou quand elle eft couverte d'une eal abondante, quoique tranquille, ne l'altère pas du tout: ainfi, fi l'air qui paffe fur des marais couverts d'eau, eft vicié, c'eft moins par des exhalaifons délétères, que par l'humidité dont il fe fur. charge. Les excrémens des animaux, quand ils font frais, ne l'altère paś; il eft au contraire vicié fur un terrain gras, quand la fécherefe eft trop forte \& trop conftante. La terre qui s'entrouvre, fournit aiors des miafmes qui, venant de l'intérieur, font plus grofiers, plus nuifibles que l'air de la campagne, même d̀ nue légère diftance, dans la proportion de $\sigma_{2}$ à 57 . Les immondices des rues l'altèrent comme 60 à 50 , fi elles font humides, \& plus légèrement quand elles font sèches. Mais rien n'altère l'air d'une manière plus forte \& plus fenfible, que la fange des marais quand elle eft légèrement humectée \& que l'air eft chaud: cette altération eft comme $\sigma_{2}$ à 34 , \& rien n'eft fi pernicicux que l'air qui entoure ces marais, quand ils commencent à fécher, ou quand il pleut en petite quantité fur des terrains gras, quand ils font légèrement humectés après une longue fécherefle. Les végétaux pourris, dégraient l'air autant que les animaux putréfiés: fatal cffet des miafmes qui s'en élèvent, rendent l'air méphitique, \& lui donnent une qualité peftilentielle. (Note de M. Burel 
mieux dévoilée; mais quelques aperçus chimiques nous permettent d'augurer que leurs élémens consistent dans une combinaison du gaz hydrogène, du gaz acide carbonique ( air méphitique), et du gaz azotique (air phlogistiqué) , joints peutêtre avec une portion de gaz ammoniacal ( air alkalin ) (I) : débris abondans de la putréfaction végétale et marécageuse (2); et leurs produits constans se réduisent à la génération dès fièvres

dans le 2 vol. du journ. de Méd. militaire pag. I3 I. Elle répond aux afiertions du Doßteur Alexander, qui a difculpé l'air des marais avec des fauffes conclufions, tirées d'expériences vraies).

(I) Voyez notre Mémoire fur les effots de l'air marécageux fur l'économie vivante, pag. $4_{4}, 53$ \& fuiv.

(2) M. de Fourcroy a fait des expériences très-luminenfes fur le gaz hydrogène des marais. Cet habile Chimifte a vu que les fonds des eaux, où pourriffent beaucoup de matières végétales, fournịfent un gaz peu inflammable \& mêlé ele beaucoup de gaz acide carbonique, que les mares $z$ toutes les eaux Atagnantes cui nourrifent beaucoup d'infectes, \& au fond defquelles leurs cadavres pourriffent, donnent le gaz le plus inflammable, parce qu'il contient une moindre portion de gaz acide carbonique. M. de Fouraroy a recueilli de certaines eaux un gaz qui, au lieu de s'alumer, éteint an contrairc la flamme, parce qu'une furabondance de gaz acide carbonique rendoit le gaz incombuftible. Nous nous abftenons d'offrir les conjectures qui pourroient -émaner de ces faits appliqués à l'étiologie des fièvres intermittentes \& rémittentes. 
dans les fièvres rémittentes.

intermittentes et rémittentes, d'autant phus graves, putrides, malignes et compliquées, que les matières fermentescibles des marais sont plus abondantes, et tendent plus à la putréfaction végéto-animale, que le miasme aérien virulent; que ces matières constituent, est plus copieux et plus concentré ; enfin, que les circonstances qui accompagnent son absorption, son développement dans les corps qui le reçoivent, sont plus aggravantes ou plus propices. On sait qu'en général un miasme est d'autant plus nuisible, qu'il trouve dans lair et dans les animaux sur lesquels il se répand plus de chaleur et d'humidité pour sa germination.

- Nous ne chercherons pas à prouver que l'effer du levain marécagéux est de produire déterminément des fièvres de nature rémittente ou intermittente. Depuis long-temps les bons observateurs ont confrmé la vérité de cette proposition; qu'ilnous suffise d'en tirer deux conséquences. La première c'est que la canse natérielle de ces fièvres étant identique, les fièvres rémittentes n'ont lieu que parce que le levain des intermitsentes est doué d'une plus grande activité, soit par des circonstances inconnues et propres au levain, soit par des puissances occasionnelles qui influent autant sur la virulence du levain que sur les dispositions de ceux 
qui le reçoivent. Et comment nous refuserionsnous à admetre cette identité de levain des fièvres intermittentes et rémittentes, ou du moins à voir dans cotte assertion une certitude présomptive; Io lorsque mille exemples nous démón rent que l'évaporation d'un marais produit dans les années et les saisons excessivement chaudes et humides, dans des sujets mal-sains, et très-disposés, des fièvres rémittentes, malignes, pétéchiales, pestilentielles, tandis que, dans la températire moins corruptive d'une autre année, d'une autre saison, d'une autre contrée, dans des individus plus sains, moins disposés, elle ne produit que des fières intermittentes et rémittentes plus ou moins simples et facheuses; 20. lorsque nous savons que les épidémies de fières rémitrentes sont précédées par des fièvres intermittentes, ou que celles - ci sont contemporaines des autres; $3^{\circ}$. que dans les cas où ces circonstances n'ont pas. lieu, on trouve, ou que la constitution n'est pas légitime et que lintempérie est extrême, ou que la saison des fièvres printannières est trop avancée, ou que la saison des fièvres automnales ne l'est point assez : car suivant une expérience constante, le froid rend intermittentes les fières dont le type étoit rémittent, tandis que la chaleur rend rémintentes les fièvres dont le type étoit inter- 
dans les fièvres rémittentes.

mittent, ou les fait bientôt aboutir à une crise complette; $4^{\circ}$. enfin, lorsque nous ne pouvons ignorer que, dans le cours d'une épidemie, les fièvres intermittentes simples et ordinaires prennent indistinctement le caractère des tierces, des doubles tierces, desquartes, enfindes continuies rémittentes, non seulement dans les différcns individus, mais successivement dans les mêmes; iorsque nous ne pouvons ignorer que les fievres rémittentes commencent souvent par être intermittentes, et finissent plus souvent encore, lorsqu'elles ont paru sous la forme de rémittentes, par se décomposer et se résoudre en intermittentes; phénomène qui, ce semble, seroit constant, si, d'une part, le levain n'étoit pas trop actif pour laisser une apyrexie entre les exacerbations, et si, de l'autre, la crise de la fièvre rémittente étoit assez imparfaite pour laisser après elle de quoi fournir aux accès d'une fièvre intermittente.

Quant à notre seconde conséquence, si le levain des fièvres intermittentes et rémittentes est de mêrne nature, et que les modifications et l'activité de ce levain fasse toute la différence dans la formation des fièvres rémittentes, il suit que ces maladies doivent en général être ascompagnées d'accidens graves, parce qu'il est de l'essence d'une maladie dégé- 
44

De l'usage du Quinquina

nérée, d'avoir une marche plus destructive, ou d'une cause morbifique renforcée, d'avoir des effets plus meurtriers. Aussi s'est - on convaincu que la marche continue rémittente est affectée au plus grand nombre des fièvres malignes (I); et a-t-on vérifié que, dès que les exacerbations d'une fièvre, qui, ayant débuté sous le type d'intermittente, tierce ou double tierce, s'est changée en rémittente, perdent l'indice assiré (le frisson au commencement des paroxismes) du type intermittent, de telles fièvres sont d'un mauvais présage et qu'elles développent très-communément les symptômes les plus dangereux (2).

Ainsi l'analogie qui se trouve entre l'ordre des fièvres intermittentes et celui des fièvres rémittentes, devient de plus en plus manifeste. De Sauvages fait remarquer, comme un trait saillant de cette analogie, que les fièvres continues du premier ordre n'ont qu'un seul redoublement, lequel arrive dans le second stade de la maladie, au lieu que dans les rémittentes, les paroxismes reviennent en tout temps; savoir : au commencement, dans l'accroissement, l'état et le déclin, ce qui leur est com-

(1) Voy. de Haen, ratio medendi, tom. IX, pag. 6.

(2) Voy, le Roi, du progn. pag. 69, aph. $3 \mathrm{I} 7$. 
dans les fièyres rẻmittentes.

mun avec les accès des fièvres intermittentes. Mais le type des exacerbations donne encore plus de force à cette analogie ; il peut être rapporté à quelques accès des intermittentes, soit tierces, quartes ou quotidiennes, simples ou composées.

XVI. On appelle quotidienne, la fièvre dont les exacerbations régulières paroissent tous les jours. Elle a quelqu'analogie avec la double tierce ( $\$$. XXI.) et la triple quarte ( $\$$. XXIII.); mais toutes ces maladies ont des nuances remarquables qui expriment leurs caractères respectifs. Arrêtons nous à ceux de la fièvre quotidienne qu'on sait avoir été appelée par les auteurs grecs du nom d'amphimérine.

Cette maladie, dont il semble que les humeurs crues, muqueuses, la pituite en un mot, si l'on veut parler avec les anciens, soient la cause secondaire, puisqu'elle attaque de préférence les enfans, les tempéramens pituiteux, les complexions humorales, qu'elle règne surtout à la fin de l'hiver, dans les saisons froides et humides, dans les temps pluvieux, se distingue dans les premiers temps du paroxisme, par le froid qui n'est simplement que horrifique (I) et qui commence au bout de quelques.

(I) L'horror, l'horreur, ou le friffon à la peau, dit 
unes des extrémités, comme au bout du nez ou au bout des doigts, sans saisir à la fois toutes les parties du corps; dans le second temps pär la chaleur qui est halitueuse, et dont l'obscure âcreté n'est sensible qu'après une assez longue application de la main sur la peau ; dans le troisième temps, par l'absence des sueurs remplacées par des déjections muqueuses ou chargées de glaires. Ces signes sont essentiels a la fiève quotidienne, et ils répondent assez exactement aux épiphénomènes qui l'accompagnent. En effet, on voit que', dans le premier temps, les malades se plaignent d'un sentiment de froid gravatif; leur pouls, qui n'a ni ordre, ni régularité, est très-petit; la langue n'est pas sèche; il n'y a point d'altération; et les vomissemens qui surviennent, évacuent des matières pituiteuses. Lorsque la chaleur se développe, elle ne le fait que par gradation bien ménagée, ne

Galien, de fymptomatum caufis, eft une maladie qui confifte dans un mouvement inégal, ou une concuffion générale de la peau, ainfi que le rigor, la rigueur ou le friffon proprement dit, dans une agitation inégale de tour le corps; mais ces deux affections font diftinguées en ce que l'horror eft un mouvement léger, \& le rigor un mouvement grand \& violent; d'où nous devons inférer que l'horror n'eft autre chofe qu'un rigor léger, un état moyen entre le rigor \& le refroidifiement. 
dans les fièrres rémittentes.

monte pas bien haut, n'oblige pas le malade à chercher le frais, à se découvrir, ne rend pas son haleine brûlante, sa respiration grande et fréquente; elle n'allume pas la soif; et le pouls qu'on observe est presque concentré, et semble ne s'élever qu'avec peine; dans ce mệme temps le corps estlourd, il y a beaucoup de propension au sommeil, la bouche est humide; à la suite du paroxisme, les urines sont, ou blanches et tenues, ou épaisses et troubles, ou rougeâtres; les déjections sont crues ct pituiteuses;pendant la rémission le visage est pále, comme bouffi, les précœurs sont élevés au-dessus de l'état naturel; la plupart des malades les ont enflés et tendus sans douleur. Telle est la marche naturelle de la période d'une fièvre quotidienne. Les reprises commencent vers le soir ou sur le matin; elles sont longues, souvent subintrantes; s'il survient du délire, c'est un délire morne. On a vu cependant une fièvre quotidienne accompagnée d'une cécité périodique, pendant laquelle limagination s'exaltoit considérablement, et causoit une loquacité continuelle ( $\mathrm{I}$ ).

On s'est convaincu que les fièvres épidémiques de ce type, ordinairement déterminées par

(1) Voy. les additions franconiques à la Médecine par M. Scheidemantel. 


\section{rv De l'usage du Quinquina}

une intempérie froide et humide, une constitution variable, sont de la classe des fièvres jentes, nerveuses. Les fièvres malignes proprement dites, maladies presque toujours sporadiques, ont aussi ce type de quotidiennes, lorsqu'elles sont de la classe des rémittentes.

XVIII. Sous le nom de tierce, on désigne la fièvre dont toutes les reprises ne viennent jamais sans laisser un jour d'intervalle entre elles; et quoique ce type soit assez pathognomo. nique pour donner un caractere à cette maladie, elle a cependant quelques indices propres à la faire reconnôtre; on lui trouve ceux qui suivent.

Dans le premier temps du paroxisme, le froid est rigoreux, et ce sentiment qu'il fait éprouver, est celui qui paroitroit comme piquer et percer le corps; dans le second temps, la chaleur qui se développe rapidement, est grande, seche et âcre d'abord au toucher ; dans le troisième temps, le relâchement est général, les sueurs et les moiteurs sont universellement répandues; et à leur défaut, il y a des selles bilieuses, des urines jaunâtres. Aussi les accidens qui marchent du pair avec de pareils simptômes, présentent une exacerbation de fièvre tierce sous un aspect orageux. D'abord le pouls est petit, foible, tardif et rare, ou sa lenteur approche 
dans les fievres rémittentes.

approche beaucoup de l'état naturel, mais il devient dans peu, petit, accéléré, fréquent et régulier; la matiere du vomissement est jaune ou verte, très-amère. Le frisson est à peine fini, que le chaud acquiert pour ainsi dire dans l'instant sa plus grande intensité. Cette chaleur également répandue, imprime à la main qui touche le malade une sensation piquante, mais en laissant quelque temps la main sur la peau, cette sensation s'affoiblit, parce que cette chât. leur n'est pas celle de la putridité. Le pouls, plus ou moins fort, est toujours bien réglé ; la soif est vive et les malades ont sur - tout envie de boire froid, la langue et le corps sont trèssecs vers l'apogée, \& quand la soif commence à s'éteindre par la boisson, il s'élève de la peau dés malades beaucoup de vapeurs chaudes qu annoncent quela sueur est prochaine; après quoile ventre se détend, les malades vont à la gar de-robe ou vomissent librement de la bile, et lurine paroît bilieuse. Pendant la rémission * la bouche est chargée d'un limon jaune et épais l'urine d'un rouge foncé pendant la chaleur; continue à être safranée; et l'on a observé que la nuit qui suit le paroxisme est assez bonne, mais que celle qui le précèdle est agitée, collpée par de fréquens réveils, et toujours les malades se réveillent avec un sentiment de $\mathrm{p}$ ?

D 
santeur, la langze plus chargée et plus sale; la bouche plus mauvaise ef une chaleur plus marquée que le jour d'auparavant.

Ceux qui sont d'un tempérament bilieux, et dans la vigucur de liage, sont les plus disposés à cette fievre, sur-tout 'quand les veilles, la diète et les trạaux ont précédé ; lorsque les malades ont été exposés aux chaleirrs de l'été, qu'ils ont beaucoup fatigué, ou éprouvé de piriquićtude et de la tension dans l'esprit. Les anciens pensoient que quand ces causes avoient un plus grand degré d'activité, elles produsoient plutôt la fière ardente. La fièvre tierce règne plus communément sur la fin du printemps, en été, au commencement de l'automne, et l'on croit que ses paroxismes commencent entre neuf et onze heures du matin: observation qui ne peut être vraie que relativement atu premier redoublement qui commence la maladie. Tout indique donc qu'elle est secondairement occasionnée par des humeurs bilielises, ou du moins qu'elle produit, plus qu'aucune autre, une dégénération bilieuse des humeurs, et que le foie est toujours ou le foyer du mal, ou le point de direction des humeurs morbifiques. tes redonjemens en terce, dit M. Menuret (I),

(I) Recueil d'obfo de Méd. des hôpit. milit. tom. II, pag. I 84. 
dans lee fievres reminttentes

annoncent une sorte d'influence du foie; ce qui le confirme, c'est que, dans ces maladies, l'hypocondre droit est prominent ou tendu, dur ou douloureux ; la jaunisse en est un symptôme ordinaire.

Ce diagnostic affecté à la vraie tierce, varie à quelques ćgards, quand cette fièvre est illégitime ou irréguliere et fausse, dans le sens de quelques auteurs. La tierce illégitime, maladie le plus souvent épidém:que en automne, commence par un frisson qui est plus léger et plus court que dans la régulière; mais la chaleur qui succède à cet état est beaucoup plus vive et plus longue : la langue est sèche, la tête fort douloureuise, les sueurs sont très-modiques, et elles soulagent peu les malades. Les paroxismes se répondent rarement, ils sont très-prolongés et les rémissions courtes et accompagnées de mal-aise et de plusieurs autres symptômes plus ou moins sérieux. Cette fièvre peut passer commie l'effet d'une disposition à plusieurs maladies humorales, graves, et en-i tre autres aux fièrres putrides.

Les paroxismes de la fièvre tierce ; quelle qu'elle soit, durent de douze à vingt-quatre heures. Lorsqu'ils s'étendent plus loin, c'est-ì dire de trente-six à quarante-huit heures, las maladie reçoit le nom de tierce prolongée, ex

$$
\text { D } 2
$$


52

De l'usage du Quinquina

suivant Celse et ses partisans, de fièvre hémitritée. Ceîte dénomination de Celse -est puisée dans les anciens qui appelloient indistinctement hémitritées, toutes les fièvres qui marchoient en tierce, et dans un sens plus général, tritéophyes. Toutes ces maladies ont la marche la plus aiguë, et leur caractère est très-malin lorsqu'elles règnent ou sont occasionnées par des intempéries chaudes et sèches, ou des consti: tutions austrines.

XVIII. Sa fièvre quarte nommée tétartophie; observée par Lautter, et que nous avons vue ici parmi les maladies de l'automne 1784 , est celle qui donne un paroxisme tous les quatre jours inclusivement, laiffant un intervalle de deux jours de rémission. Cette diversité de type annonce encore une diversité dans la marche des symptômes. En effet, le froid qui constitue le premier temps n'est ni horrifique ni rigoreux, et les malades se plaignent d'une lassitude dans les os, et d'avoir en même temps les chairs coutuses, pilées, froissées. Ce froid est long et les malades craignent de ne jamais se réchauffer; le pouls est foible, tardif, et tellement rare, qu'il ne ressemble à aucune autre affection; on diroit que, dans le fort du froid, l'artère est comme liée, attachée et retirée dans lintérieur des chairs, sans pouvoir s'élever. La 
chaleur qui forme le second temps est de phis ou moins de durée, et n'est pas à beancoup pres aussi vive que dans la tierce, ni le pouls autant développé. Le dernier temps est souvent trèslong, les crises qui le terminent sont mediocres et les urines plus crues que dans les autres rémittentes.

Une automne d'une température froide, sèche et inégale, produit pour l'ordinaire ces maladies qui attaquent ceux qui ont passé la vigueur de leur jeunesse, et dar.s lesquelles l'hypocondre gauche paroît plus ou moins afrecté: ce qui donne quelques probabilités à l'opinion des anciens, qui croyoient que ces fièvres étoient causées par une humeur atrabilieuse.

XIX. Tels sont les types et les caractères qui différencient les trois espèces majeures des fièvres. Mais l'assimilation, la conjugaison de plusieurs espèces constituent d'autres variétés. Quand la nature n'est point trop dérangée, elle unit ensemble les fièvres d'un caractère et d'un type analogue; ainsi se forment les doubles quo. tidiennes, les doubles tierces, les doubles quar tes, les triples quartes, les doubles tierces doublées, les triples tierces triplées; mais la nature est quelquefois plus accablée, et dès-lors, en se réunissant, les fièvres d'espèce différente présentent deux ordies de mouvemens distincts, qui

$D_{3}$. 
indiquent assez la surcomposition de la maladie. Dans cette classe sont les hémitritées et les continues rémittentes; car nous affectons ce dernier nom aux fièvres composées d'une fièvre véritablement continue et d'une fièvre réellement rémittente.

$\mathrm{XX}$. La double quotidienne a deux paroxismes en vingt-quatre heures, ordinairement dinégale intensité, d'inégale durée; et lorsque ce type a lieu, il est bien rare quela fièvre ne soit pas subintrante. On la distingue de la double tierce doublée ( $\$ X X I V)$ ct de la triple quarte triplée, parce que les paroxismes ont tous les jours la même longueur, la même violence qu'on avoit remarquée dans les exacerbations de la veille.

XXI. Dans la double tierce, qui forme une maladie très-commune, on trouve, comme dans la quotidienne, un paroxisme chaque jour; mais outre que les symptômes de ces deux fièvreș sont différens, il y a encore de particulier que les reprises sont inégales; la première répond pxactement à la troisième, et la quatrième est̂ en tout analogue à la seconde. Ces deux exacerbations, qui, presque toujours, occupent chacune un jour, peuvent tomber dans le même jour, tandis que le lendemain est accordé en entier à la rémission. Autrement la double tierce n'a rien dans ses symptômes, qui la fasse diffé 
rencier de la tierce, â la nausee près, qui est plus commune dans celle-ci pendant le frisson. Il est encore três-certain que la double tierce a souvent plus de râpport avec la tierce illégitime qu’avec la régulière.

XXII. La dónble quarte donne en trois jours deux exacerbations qui peuvent se ranger de decix matières. Par exemple, le lundi et le mardi ont chacun une exacerbation, le mercredi est jour de rémission, le jendi et le vendredi sont des jours paroxistiques et la reprise du lundi repond à celle du jeudi, tandis que celle du vendredi ressemble à celle du nardi. Cette maladie est, dit-on, très-commune en Hollande. Notis avons vu la double quarte sous un autre type. Le lundi avoit une exacerbation, le mardi étoit jour de rémission, et le mercredi avoit un paroxisme qui ne ressembloit pas à celui du lundi; mais le jeudi donnoit cette exacerbation analogue à celle du lundi, le vendredi étoit encore jour de rémission et le samedi donnoit une reprise égale à celle du mercredi. Les deux reprises peutvent arriver le même jour.

XXIII. La triple quarte a lieu lorsque, chaque jour étant occupé par une exacerbation d'inégale force et d'inégale longueur, la première répond à celle du quatrième jour, la seconde à celle du cinquième jour, et la troisième à celle du sixième 
jour, ou lorque les trois reprises viennent dans le même jour, les deux autres se passant danș la rémission, à l'instar de la triple quarte intermittente dont Rivière nous a fourni un exemple.

XXIV. La double tierce doublée a, comme la double quotidienne $(\$ X X)$, deux périodes par jour; mais ses exacerbations se correspondent comme celles de la double tierce ( $\$ X X I)$, de manière que la première exacerbation du premier jour répond à la première exacerbation du troisième jour, tandis que la seconde exacerbation du premier jour correspond à la seconde exacerbation du troisième jour, comme ce qui arrive dans le second jour est conforme avec ce qui arrive le quatrième. Cette fièvre double tiêrce doublée a été observée par Rivière, et décrite sous le nom d'espèce rare d'hémitritée; elle a été vue enrore par M. Majault, médecin de Paris: ce qui n'empêche pas que cette maladie ne soịt très-rare et communément subintrante. Il ạrive plas souvent que la double tierce soit triple, c'est-à-dire qu'elle ait un jour deux paroxismes distincts et le lendemain un seul paroxisme. C'est à cctte fièvre que quelques anteurs, nottamment Rosen et Charles le Roi, ont affecté la dénomination d'hémitritée. Brendel et Skenkius 
dans les fièvres rémittentes.

ont décrit la même maladie sous le titre de triple tierce.

$\mathrm{XXV}$. La double quarte seroit pareillement doublée, si on observoit deux paroxismes chaque jour des deux jours paroxistiques, se correspondant régulièrement l'un avec l'autre.

XXVI. Enfin la triple quarte doublée auroit lieu, lorsque les exacerbations seroient au nombre de deux chaque jour, se répétant, de manière que le premier paroxisme du premier jour répondroit au premier paroxisme du quatrième jour, que le second paroxisme du premier jour seroit égal à la seconde reprise du quatrième jour ; en étant de même pour les exacerbations du second et du cinquième, du troisième et du sixième jour.

XXVII. D'une fièvre quotidienne et d'une tierce, unies ensemble, et dont par conséquent les paroxismes sont très-violens de deux jours l'un, puisque la reprise de la quotidienne et celle de la tierce coincident, il résulte une maladie composée, à laquelle Galien imposa le nom d'hémitritée. Cette fièvre ne semble, au premicr aspect, qu'une double tierce ( $\$ \mathrm{XXI})$ très-grave; cependant, à la bien considérer, on lui trouve la marche d'une maladie particulière. En effet, dans la double tierce où il y a aiternativement une forte exacerbation et une autre qui l'est moins, 
le froid du premier temps, dans l'un et l'autre paroxisme, est rigoreux. Dans l'hémitritée, au contraire, où les exacerbations sont pareillement l'une plus forte, l'autre plus foible, on trouve que la grande reprise débute par un froid qui tient de l'horreur et de la rigueur, parce que le grand frisson de la fièvre tierce est en quelque sorte adouci par l'influence de la fièvre quotidienne, ce quii lui a mérité le nom de sémi-tierce ou demi-tierce, tandis que l'exacerbation du lendemain commence par le froid simplement horrifique, qui constitue la fièvre quotidienne. Mais cette exacerbation, quoique moins forte que celle du jour précédent, quant à l'intensité des symptômes, n'en est pas moins plis longue que le grand paroxisme, tandis que la foible reprise de la double tierce est réellement et moins intense quant aux symptômes, et d'une moindre durée. L'hémitritée commence quelquea fois par la quotidienne, et alors les grandes reprises se trouvent dars les jours pairs; d'autre fois cette fière débute par les grands paroxismes, et fixe par conséquent les forts redoublemens aux jours impairs. Il n'est pas rare cependant, lorsque cettc fièvre est mal traitée ous qu'elle est d'une nature très-mauvaise, que les. exacerbatio ns après avoir marché en jours impairs, passent aux jours pairs et réciproquementa 
dans les fières rẻmitientes:

Si l'hémitritée se décompose, comme Galien en rapporte un exemple, le malade aura chaque jour une exacerbation de fièvre quotidienne trèsdistincte, et, de deux jours l'un, un paroxisme de fièvre tierce qui, selon sa nature légitime ou irrégulière, connera à la maladie une marche caractéristique.

L'hémitritée paroît plus communément dans l'âge viril, et quand on approche de la vigueur ou fleur de l'âge. Fontanus dit qu'elle attaque principalement les nobles, les ecclésiastiques, les courtisans, les jeunes femmes, et ceux qui vivent dans l'oisiveté et dans la bonne chère. Elle paroît vers la fin de l'été et en automne, plus fréquemment dans les pays chauds, humides et exposés aux vents du midi. Les constitutions austrines peuvent la répandre par-tout. On sait qu'elle a été endémique à Rome, depuis Galien jusqu'à Baglivi et Orlandi (I), apparemment à cause de l'air chaud, marécageux, et de l'exposition méridionale de cette contrée.

XXVIII. On ne trouve guères d'autres conjugaisons des fièvres d'un type et d'un caractère

(1) De exficcandarum paludum pontinarum utilitate, deque infirmitatibus qua ab aquis fagnantibus exoriuntur dissertatio, cui altera accedit de morbis ab amo 1778 ad $I_{7} 8$, Rome observatis, In-80. 1783 . 
60

De l'usage du Quinquina

différens; mais il n'est que trop ordinaire de voir une fièvre rémittente entée, et dominant sur une continue, et réciproquement. Une pareille combinaison rend la marche de la maladie très-anomale, les rémissions sont imperceptibles et marquées par les symptômes propres à la fièvre continue; les paroxismes sont également moins marqués, et leurs premier et troisième temps sont on ne peut plus obscurs; aussi lorsqu'on parvient à dissiper la fièvre rémittente par les remèdes qui lui sont convenables, la fièvre continue poursuit souvent son cours et sans se déranger, tant la nature est fixe dans les déterminations que certaines causes lui font prendre, ces déterminations fussent-elles très-différentes. $V$ an Swieten rapporte l'histoire d'une fièvre quarte que ne troubla en aucune manière l'intervention d'une pleurésie. Nous avons vu une fièvre quarte de treize mois, se compliquer sans interruption avec une fièvre putride continue qui survint au troisième mois d'une grossesse, et n'être pas dérangée par la révolution de l'accouchement qui s'effectua au septième mois de la gestation.

Les fièvres continues rémittentes, dans le sens que nous leur attachons ( $\$ X I X)$, sont des maladies plus communes qu'on ne le croit, et cela doit être, si les idées que nous avons hazar* 
dans les fièvres rémittentes.

dées sur les causes ( $\$ X V$ ) qui produisent les fièvres intermittentes, rémittentes et continues, peuvent être vérifiées. C'est en vertu de leur dominance respective, 'qu'une fièvre visiblement continue se change au milieu de son cours, après une bonne application des remèdes appropriés, en fièvre distinctement rémittente, et qu'une fièvre dont le type aura d'abord ćté rémittent, prend une tournure véritablement continue, sans que son caractère se déprave: ce qui auroit inévitablement lieu, si ce changement étoit l'effet d'une méthode inconsidérée. Remarquons encore que la complication d'une fièvre continue et d'une fièvre rérnittente s'observe assez souvent pendant les épidémies de fièvres continues dont les rémittentes constituent la maladie intercurrente, et réciproquement lorsque les fievres rémittentes forment la maladie épidémique ou dominante.

XXIX. D'après ce que nous avons dit ( $\$$ XVI à XXVIII) sur le type des fièvres rémittentes, on voit qu'à raison de ce type, les fièvres rémittentes sont simples ou composées: simples lorsqu'il n'y a dans une période de fièvre quotidienne, tierce ou quarte, qu'une seule exacerbation régulière, illégitime ou subintrante (I):

(1) Toute fièvre subintrante est esseutiellement de la famille des rémittentes, c'est le fentiment de Senas (de 
62

De l'usage du Quinquina

composées, lorsqu'il se trouve, dans une pé-t riode de fièvre quotidienne, tierce ou quarte, une ou plusieurs reprises légitimes, irrégulières ou subcontinues; le fond de la fièvre étant dı caractère rémittent ou du caractère continu. Mais tous ces types ne font qu'exprimer la marche de la maladie; il ne fournissent pas de grandes indications pour le traitement; c'est plutôt di génie ( $\mathrm{I}$ ) de la maladie que dérivent les indica-

recondita febrium, etc. pag. I 54. ) Sauvages, Colombier \& autres. Suivant Sauvages la quotidienne subintrante eft une quotidienne rémitténte; \& toute quotidienne doubie, à plus forte raison la triple dont parle Primerose, appartient aux fièvres du caractère rémittent. Les fièvres décrites dans les auteurs sous le titre de subcontinue, de subintrante, ne méritent pas, suivant $M$. $C_{0-}$ lombier, de refter parmi les intermittentes, puifque ces maladies sont de vraies rémittentes putrides ou malignes, comme l'indiquent assez les symptômes qui les accompagneat.

(I) Nous entendons par génie d'une maladie, la disposition, l'aptitude, le penchant qu'elle a vers tel état plutôt que vers tel autre; vers l'état d'inflammation, par exemple, préférablement à celui de putridité. Il ne faut pas confondre ce terme avec celui de nature de la maIadie, auquel nous attachons un sens différent. Une maladie d'une nature inflammatoire, est celle dont les simptômes essentiels ont actu un caractère d'inflamma tion qui décèle la natu:e de cette maladie; au lieu que 
rions, et ce génie peut être inflammatoire, putride ou bilieux.

XXX. Dans les fièvres du génie inflamman toire, les forces vitales paroissent augmentées, loin d'être affoiblies. Le pouls est habituellement étendu, développé, quelquefois petit; mais, dans l'un et l'autre cas, il y a de la force. Le pouls concentré devient plein et fort par la saignée. La chaleur de l'habitude du corps, la soif, le mal à la tête, le délire, la difficulté de respirer, en un mot tous les accidens qui peuvent survenir, répondent à-peu-près à la violence de la fièvre, au degré de fréquence, de force et de dureté du pouls. Ces fièvres n'abattent pas subitement les forces animales. Si le pouls y devient mou, foible, ou ce symptôme tenant à quelque cause passagère, il ne dure pas, ou sil persiste, c'est parce que la vie commence à s'éteindre par l'effet d'une affection grave et irrémédiable de quelque viscère. A ces indices

les symptômes essentiels d'une maladie qui a un génie inflammatoire, ne sont pas inflammatoires actu; mais s'ils s'élèvent à un degré plus fort, ils prennent pour lors un caractère d'inflammation préférablement à tout aurre. Voy. Mém. de la Soc. Roy. de Méd. to I , p. 5s, des Mém. Note m. 
non équivoques et essentiels du génie inflammatoire, se réunissent une infinité de symptồ mes accessoires, dont l'ensemble, en venant iे l'appui du diagnostic, forme le tableau de la période d'une fièvre rémittente inflammatoire. Parmi ces symptômes accessoires, on trouve, avant le paroxisme, une douleur de tête et des orbites, accompagnée d'une pesanteur douloureuse à l'estomac, de lassitudes spontanées et de sensations alternatives et irrégulières de frisson, de chaleur et quelquefois de sueur. Le visage est rouge, les vaisseaux du blanc de l'œil sont gorgés de sang; il y a sur la langue un tapis mince, argentin, sec, et les bords en sont très-rouges. La respiration est laborieuse, le ventre tendu, la région épigastrique ou hypocondriaque sensible, ou légèrement douloureuse par la pression. Le frisson se déclare, il est court, souvent même peu marqué, et le pouls est fort, dur et serré; il se développe ensuite pendant la chaleur, et il devient grand, plein et très-dur jusquà la fin de l'exacerbation qui dure de is à 18 heures, avec beaucoup d'ardeur, beancoup de soif, beaucoup de mal de tête. Dans la rémission, le pouls est moins plein, mais il consorve un caractère de force et de dureté, qui indique visiblement l'état des solides et celui des fluides; car, pendant le paroxisme, 
dans les fièvres rémittentes.

xisme, le pouls plein et grand, peut être l'êres de la raréfaction du sang; dans la rémission au contraire il marque le véritable point des choses. Les urines sont troubles ct rouges; si elles restent claires, on est sij t à l'témorragie du nez. Au lieu de l'hémorragie, il se fait dans quelques malades une éruption d'échauboulures ou de petites taches ronges ressemblantes à celles qui sont l'effet des morsures de la punaise. Cette éruption n'est que symptomatique, elle parồ ordinairement durant chaque exacerbation, disparoit pendant la rémission, et revient jusqu'd ce que les secours aient enlevé la cause du tumulte des humeurs.

De telles fièvres sont des maladies de prirtemps, sur-tout lorsqu'il succède à un hiver froid et sec. Elle attaquio de préférence les tempéramens vigoureux et pléthoriquəs, les jeunesgens qui ont fini leur crue, principalement les jeunes femmes qui sont grosses. Elles sont communes dans les pays hauts et secs, éloignés de la mer, et elles forment la constitution épidémique de l'année, lorsqu'il existe une intempérie véritablement printanière.

C'est aux fièvres rémittentes du génie inflammatoire que nous rapportons la synoque non putride des anciens, la grande fière de la nature ou la fière dépuratoire de Sydeníam, la 
fièvre rémittente des camps de Pringle, la fièvre rémittente de Rochefort de M. Retz; observant que ces maladies participent ensuite plus ou moins du caractère putride ou bilieux, selon qu'elles reçoivent plus ou moins d'influence de la dégénération propre aux maladies d'été et de l'automne; ajoutons que les fièrres humorales prennent deux fois l'année le genie de l'inflammation: savoir, au printemps, lorsque la constitution bilieuse s'établit sur les débris de la constitution inflammatoire de l'hiver, et dans l'automne, lorsque la constitution inflammatoire s'établit sur les débris de la constitution bilieuse.

XXXI. Le génie putride opposé dans tous ses chefs à celui de linflammation, se reconnoît à l'odeur fétide des excrémens, des sueurs et du malade, à la chaleur âcre et mordicante qui, dans tous les temps, à l'exception de l'invasion du paroxisme, est plus ou moins sensible; au limon épais et sale dont la langue est chargée, et qui dégénère bientôt en une croûte sèche, dure, brune ou noire; au goût détestable de la bouche; au dégoût affreux pour les alimens, tirés du règne animal; enfin, à tous les épiphénomènes qui dérivent de l'alcalescence des sucs, et de leur tendance à la putréfaction, de leur dégénération plus ou moins rapide : tels sont l'abattement des forces, la disposition à la 
dans les fièvres rémitientes.

diarrhée, aux sueurs, le météorisme du ventre, les légers soubresants des tendons, etc. Ici les saignées ont un effet dangereux; les déjections putrides sont sans consistance, les urines fétides n'ont point de sédiment, et les humeurs dissoutes ou dissolubles rendent les pétéchies ou les hémorragies symptomatiques très-communes. La vraie constitution putride est épidémique, et dépend, entr'autres causes capables d'altérer les humeurs, des chaleurs vives de l'été, sur - tout lorsque l'humidité vient renforcer cette diathèse putride. Les fièvres rémittentes putrides attaquent autrement ceux qui ablisent ou font un long excès des viandes très-noumissantes et faisandées, ceux qui mangent des niets succulens. Elles attaquent encore le peuple qui mange des substances gâtées, boit des liqueurs tournées, habite des séjours mal sains. Les enfans quion élève mal, qu'on ne peut rassasier de viande, avec laquelle ils mangent peu ou point de pain, sont encore exposés aux fièvres putrides, mais portées au plus haut dégré de dissolution et de danger.

Plusieurs fièvres rémittentes, soit épidémiques; soit sporadiques, qu'on a décrites sous des noms particuliers, méritent d’être rapportées à la classe des putrides: nous nommerons seulement la fièvre subintrante, rangée, par plusieurs aulE 2 
teurs, parmi les intermittentes, quoiqu'elle soit véritablement une fièvre rémittente putride, qui se masque d'abord sous le type de la fièvre quotidienne, et manifeste, au bout de quelques jours, tous les signes de putridité. Nous nommerons encore la tierce prolongée de Torti, maladie moins trompeuse et caractérisée rémittente putride maligne, tant par les accidens dont elle est accompagnée que par la durée des paroxismes qui se touchent successivement de plus près.

Sans doute que, dans beaticoup de cas, les fièvres putrides sont telles dès leur origine, mais, dans plusieurs autres circonstances, cette dissolution putride a été précédée, ou par un état muqueux ou pituiteux des humeurs, ou par un état inflammatoire. Temoin l'épidémie de Gottingue dont Roederer et Wagler nous ont donné une bonne description; et celle de Naples dont Sarconne a fait une peinture si complette; témoin la maladie qui prit un caractère d'universalité dans plusieurs endroits de la France pendant l'été de 1781 , et sur laquelle la Société Royale de Módecine, publia d'utiles réflexions. Dans l'épidémie de Gottingue, la première stade de la maladie avoit un génie inflammatoire; mais après la coction, c'est-à dire! dans la seconde stade, 
la fièvre étoit réellement putride (I) dans la maladie décrite par la Société Royale, on voyoit évidemment le caractère d'une dissolution putride suivre et prendre la place de la densité inflammatoire, et l'on avoit à combattre les accidens de l'une et de l'autre de ces causes funestes. (2).

XXXII. Enfin, le genre bilieux prend encore une autre tournure et s'annonce par d'autres symptômes. C'est d'ábord un certain affaissement, un ennui de soi-même, accompagné de pésanteur gravative de la tête, de mal aux reins, la bouche est amère, pâteuse; l'épigastre est gonflé sans douleur; il y a des nausées, des vomituritions, des vomissemens d'une humeur très-amère, jaune, verte ou érugineuse. Les joucs sont colorées comme avec du minium, et cette rougeur fait place à une couleur d'un verd jaunâtre. $\mathrm{Ce}$ sig̣ne est très-expressif, s'il faut en croire Stoll (3)

(1) Primum quidem ftadium aliquid infammate indolis fape fibi adjungit, quice fenfim factâ coctione, folvitur nimis in putridam. De morbo mucoso, p. 09.

(2) Réfexions lues dans la féance tenue au Louvre par la Société Roy. de Méd. le 18 feptembre 178I, fur la nature de la constitution de cette année, etc. p. 2 . \& 5 .

(3) Ratio medenai, pars I, p. 42, E. alibi passim parsz. p. 86. 
190

\section{De.l'usage du Quinquina}

et M. Frank (I). Avant le vomissement, le visage des malades est très-allumé et il devient très-pâle après qu'ils ont rejeté beaucoup de bile. La saignée qui d'abord semble soulager, augmente, dans peu, tous les accidens. La langue est moite, sale, épaisse, jaune, bilieuse; le blanc des yeux est terne et les caroncules larrymales ont une couleur verdâtre. Les vomissemens repremnent à chaque invasion de frisson, et sont même quelquefois assez violens pour imiter le cholera-morbus le plus fort. A cette époque le pouls est plus ou moins plein, concentré; quand il se déploie, il peut être grand, mais il n'est pas essentiellement dur; on sent qu'il y a plutôt raréfaction du sang que pléthore. Après l'exacerbation, les urines qui étoient d'abord rouges, claires et transparentes, deviennent troubles, jaunes et chargées d'un sédiment terreux, semblable à la brique pilée. L'ictère paroît quelquefois dans le cours de la maladie, au commencement, c'est-à-dire, avant le sixième jour, il est l'effet de l'augmentation de la fièvre et de l'engorgement du foie qui, pénétré de la cause morbifique, se durcit et devient douloureux;

(1) De larvis morbortm biliofis. 1984. Voy, anfib to trạité de $M$. Reil de polichalya 1784. 
après le septième jour, l'ictère annonce la fonte humorale, et une diarrhée salutaire ne manque pas d'avancer la guérison.

Les fièvres bilieuses règnent sur la fin de l'été et pendant l'automne; elles attaqueat de préférence le peuple qui vit avec peine, les manouvriers qui sont exposés à la fraîcheur du matin, à la chaleur du jour, à l'humidité du soir et de la nuit. Ces maladies ont quelquefois une invasion trop tumultueuse, pour permettre la rénittence; mais une saignée, si elle est jugée nécessaire, ou la première évacuation ne manque jamais de la déterminer; car les fièvres bilieuses sont essentiellement rémittentes, et ce type leur est si foncièrement attaché, qu'on n'a pas bescin d'autre indice pour distinguer la pleurésie infiammatoire de la bilieuse ( 1 ); le causus bilieux du causus inflammatoire (2).

C'est le propre d'une bile très-acrimonieuse, et, pour ainsi dire, enflammée, d'exciter un érétisme dont les effets sont fort analogues à ceux que produit le génie inflammatoire ( $\$ . X X X) ~ S$. l'on prenoit le change, l'erreur seroit aussi grossière que préjudiciable au malade; on ne s'y mé-

(1) Voy. Stoll. rat. méd. pars I, pag. 47.

(2) Voy. Grant. Rech. fur les fièv. tom. I, pag. I74, 243. 


\section{De Tusage du Quinquina}

prendra pas, si l'on considere que les fièvres de ce caractère règnent après et pendant des chaleurs fortes, qu'elles sont causées et entretenues par des intempéries très-sèches et chaudes : les inrempéries chaudes et humides causent plutôt des fievres putrides et bilieuses; la fièvre ardente ou l'hémitritée des anciers étoient causées par cette bile caustique, qui produit de nos jours, dans tous les climats chauds, un vrai causus bilieux, dont la marche est si rapide, dont les effets sont si redoutés. La fièvre jaune de l'Amérique n'est pas d'une autre nature; et dans les climats tempérés, nous voyons quelquefois des diminutifs de ces maladies si dangereuses, lorsque la sécheresse et les chaleurs composent la constitution qui les engendre. Telle fut, dans la majeure partie de la France, la constitution du printemps et de l'éte en 1781 ; aussi les maladies de cette constitution furent - elles l'image de ces fières ardentes qui désolent les habitans des pays plus chauds que la France, et sujets aux infections causées par des lacs et des marais à moitié desséchés par la chaleur d'une saison brîlante: maladies si bien observées par Torti, $B a-$ fivi, Walcarcnghi, qui s'éten lirent sur les bords du Dunube, et même dans la Silézie, au comm mencement de ce siècle, et dans lesquelles l'érétisme du système membraneux est si fort au prewier période, que M. Quarin n'a pas crains de 
dans les fièrres rémittentes.

dire que ces fièvres dépendent de l'inflammation de presque tout le systeme vasculaire. Ce sont ces fievres dans lesquelles la bile joue le plus grand rôle, qui, à corsidérer leur invasion, commencent par être inflammatoires, et finissent par être putrides; elles prennent même quelquefois un caractère pestilentiel, et Galien a voulu qu'on les distinguât, par un nom particulier, des fièvres putrides ordinairer.

Disons un mot de la fièvre pituiteuse, qu'on ne confond que trop souvent avec la fièvre bilieuse, dont elle differe à tant de titres, et qui mérite bien mieux d'être assimilée avec la fièvre muqueuse. Produite par une constitution de l'air froid et humide, par un régime vicieux et par la disette de bons alimens, elle a pour symptômes distinctifs, une marche lente avec un pouls foible et intermittent, des urines claires, une langue blanchâtre, recouverte par une substance qui ne ressemble pas mal à une pièce de lard. Dans cette maladie, le sang eš surchargé d'une substance glutineuse qu'il ne faut pas confondre avec la coenne phlogistique des maladies inflammatoires, et la partie rouge es ́dans un état de dissolution; les premières voies sont inondées d'une pituite plus ou moins âcre qu'il faut distinguer de la saburre bilieuse, On seroir tenté de la prendre 
pour la fièvre lente nerveuse que Glaff et Huxam ont très-bien décrite, mais qu'il faut rapporter aux fièvres malignes ou nerveuses, et suivant Selle, désordonnées (atacta ).

XXXIII. Ainsi les saisons ayant un cours légitime, les maladies qui en sont un effet, ont successivement un génie différent; inflammatoires au printemps et dans les constitutions annuelles, préparées par de longues gêlées, produites par des vents septentrionaux, entretenues par un temps sec $(\$ . \mathrm{XXX}$.); putride en été, et dans les constitutions annuelles, précédées par des chaleurs humides, excitées par des vents du midi, fomentées par un temps humide et chaud ( $\$$. XXXII.) ; bilieuse en automne, et dans les constitutions annuelles, dévancées par une fraîcheur plutôt sèche qu'humide, secondées par le souffle alternatif des vents du nord et du midi, développées par un temps sec et chaud ( $\$$. XXXII.); enfin, pituiteuse dans le passage de la constitution printanière à la constitution estivale, et pendant les températures humides et froides. Cette dégénération inflammatoire, pituiteuse, putride, bilieuse, est si propre, si affectée aux maladies du printemps, de l'été et de l'automne, que lorsqu'une forte intempérie, lorsque des causes énergiques ont déterminé une grande épidémies 
dans les fières rémittentes. ont donné lieu à une constitution d'une ou de plusieurs années, la fièvre dominante, sans rien perdre de son caractère essentiel, admet les nuances, et reçoit les modifications que lui donne l'inflience particulière des saisons. La fièvre muqueuse de Gottingue nous en fournit une preuve; son règne fut à peu près d'environ trois ans, mais l'année $176_{1}$ fut l'époque de sa plus grande fureur, et presque à chaque mois on lui tronvoit quelque chose de particulier; aiguë, vermineuse, bilieuse ou putride et maligne avec le type d'hémitritée en janvier, inflammatoire en février; inflammatoire et pétéchiale dans le courant de mars; pleurétique, avec le type d'hémitritée et dégénération en intermittente, pendant le mois de mai ; très. maligne et soporeuse en automne, suivant ainsi, d'une manière très-rapide, les influences de l'élément mobile, d'ò̀ elle dérivoit, et qui péchant, tantôt par la chaleur, tantôt par la froi. dure, presque toujours parl'humidité, avoit déterminé la constitution catarrhale, dont la fièvre muqueuse (I) est le dernier produit, comme le plus funeste.

(I) Cette fiévre n'étoit qu'une dégénération, une sranfmutation des fièvres intermittentes. Parni nous, ds pareilles épidémies font produires par un long fouffe 


\section{De l'usage du Quinquina}

Ce n'est pas qu'il n'y ait des constitutions annuelles, qui, maintenues dans une certaine uniformité par la dépravation de l'état propre aux différentes saisons, conservent aux épidémies régnantes le caractère qui leur a été imprimé par l'intempérie, et qui ne peut être perverti que par une révolution considérable. L'épidémie bizannuelle de Laschendorf, décrite par Lautter, confirme cette assertion. Le génie de cette fièvre rémittente fut inflammatoire pendant tout le cours de l'année 1759 ; mais la température ayant changé, cette mîme fièvre eut un génie putride avec malignité durant toute l'année 1760 .

XXXIV. C'est en faisant la plus scrupuleuse attention au génie que constitue la maladie; c'est en ne négligeant rien de ce qui peut servir à la connoître, qu'on s'instruit, nous ne disons

du nord eft; vent qui porte, d'une manière marquée, fur la partie muqueufe ou lymphatique du fang; auffi, les maladies qui dérivent de cette pléthore muqueufe, font-elles principalement reconnoiffables à cette grande quantité de mucus que toutes les voies d'excrétions entraînent, à la coction purulente qu'éprouvent ces fièvres fi fufceptibles de dégénérer, en phthifie pulmopaire, et fur-tout au fang qu'on reçoir dans les palettes, et qui eft prefque tout muquelix ou fort coenneux, mais d'une coense plutôt molle que dure. 
dans les fièvres rémittentes.

pas simplement pour la guérir, mais pour la guérir de la manière la plus prompte et la plus sûre. Ajoutons ici une réflexion qui complète lidée générale que nous avons voulu donner du génie des fièvres rémittentes épidémiques; c'est, que lorsqu'une intempérie donne naissance à un fleau, celui-ci ne se développe pas toujours sous le règne, ou immédiatement après cette intemperie; témoin l'épidémie décrite par Ramazzini, et qui se répandit à Modène sur la fin de 1692 en $16_{93}$ et 1694 . L'année 1692 avoit été légitime dans toutes ses saisons; cependant la maladie étoit vraiment automnale, et dépendoit de l'année I $\sigma_{\theta}$ I , qui fut remarquable par ses intempéries. Ainsi les progrès de la dégénération des humeurs du corps vivant, suivent quelquefois lentement l'impulsion que leurs ont données des causes qui ont agi longtemps, fortement, et d'une manière universelle pour une ville, une contrée, une classe d'individus, plutôt que pour une autre.

Nous dirons encore que les maladies contemporainss contribuent à fixer le diagnostic des affections dominantes; car, ainsi que les fièvres intermittentes et les maladies périodiques sont contemporaines des fièvres rémittentes, de même les maladies inflammatoires règnent sporadiquement durant le cours des fièvres dont le génie 
est inflammatoire. On peut en dire autant des maladies putrides et bilieuses.

$\mathrm{XXXV}$. On a vu que le génie des maladies étoit relatif à l'effet des saisons, et que l'ordre des constitutions répondoit aux diverses modifications que donnent aux solides et aux fluides des corps vivans les différens temps de l'année. (5. XXX à XXXIII.) Mais la marche de ces maladies n'auroit-elle rien d'analogue à cet ordre constitutionnel? Seroit-il possible que des fièvres qui prennent successivement un génie opposé, gardassent la même régularité dans leur marche, dans leurs effets, dans leur tendance ? Déjà nous avons averti que, respectivement au type ( $\$$. XVI et XVII, XVIII et XIX), les fièvres rémittentes varioient à quelques égards, et suivoient linfluence des saisons. Nous dirons actuellement, appuyés sur l'expérience, qu'eu égard à la marche, ces fièvres prises dans la totalité des constitutions épidémiques, commencent par être intermittentes, et finissent par reprendre le caractère intermittent: autre analogie qui lie plus étroitement l'ordre des fièvres intermittentes, et celui des fières du caractère rémittent. ( $\$$. III et XV.)

En effet, le solstice d'été, qui partage l'année civile à peu près en deux parties égales, semble diviser de même en deux classes l'ordre médical des constitutions. Les maladies régnantes, 
dans les fièvres rémittentes.

en commençant à l'équinoxe du printemps, different beaucoup de celles qui sévissent et débutent à l'équinoxe d'automne, lorsque l'année est légitime, et qu'une intempérie dominante ne favorise pas le règne d'une épidémie, et son influence sur toutes les maladies intermittentes. C'est par rapport à la diversité de ces maladies, que Sydenham envisagea, comme un point de la plus grande importance, la division des fièvres d'accès en printanières et en automnales, et que Grant, le judicieux commentateur de ce grand praticien, appelle les unes fièvres du printemps, et donne aux autres le nom de fièvres de la moisson.

En suivant avec sagacité la marche de ces fièvres, nous voyons que celles du printemps, d'abord simples et régulières ( si l'hiver précédent a été légitime, autrement elles ont le caractère des fièvres d'automne) ont une trèsgrande aptitude à se changer en fièvres rémittentes et continues, ou leur cèdent la place; tandis que les fièvres de l'autre constitution, d'abord continues ou rémittentes, se décomposent et finissent par être intermittentes, ou cédent la place à de véritables fièvres d'accès.

Il est donc permis de rapprocher du même ordre, des maladies qui sembleroient d'abord exiger une exacte séparation : les unes étant des 
fièvres dégénérées. d'intermittentes, les autres étant des fièvres qui doivent revenir au caractère intrrmittent. Mais il résulte aussi de là, que quoiqu'il faille diviser les fières rémittentes de cet ordre en deux espèces, en fièvres rémittentes d'origine intermittente, et en fievres rémittentes d'origine continue; dans le fond, ces deux espèces de fievres n'en font qu'une dans la nature, parce qu'elles seroient exactement les mêmes, si les saisons et d'autres causes d'un effet analogue n'apportoient quelque différence dans la marche. Il résulte encore que le froid et le chaud (on en dit autant des causes qui agissent d'une manière semblable) sont les deux agens qui règlent la marche de ces fièvres ; la chaleur opérant leur dégénération, comme le froid leur rend la régularité. Quelques détails vont éclaircir cette matière.

XXXVI. Le printemps fait sentir ses douces influences, et les fièvres d'accès, communément quotidiennes ou tierces, se répandent. Les chaleurs augmentent, et la márche régulière des maladies n'inspirent pas l'idée de leur dégénération. Mais le temps devenant plus chaud, et sur-tout s'y joignant de l'humidité, des vents du sud, ces fièvres étant traitées par des remèdes échauffans, arrêtées par de prétendus spécifiques, on découvre les signes d'une dégénération 
dans les fièvies rémittentes. tion imminente. Flle est d'abord annoncée par la durée totale de l'accès, plus longue qu'elle n'avoit coutume de l'être; par celle du second temps beaucoup plus considérable qure celle des autres; par la briéveté du premier et du troisième, et la diminution de leur intensité. Bientôt il s'y joint le trouble progressif des intermissions. Il n'y a pas encore de la fréquence dans le pouls; mais le malade a de la chaleur, de la sécheresse à la peau, de l'ardeur dans la bouche, une soif inusitée, et ses urines sont rougeâtres sans sédiment; enfin, le rithme du pouls s'altère, et la fièvre est rémittente bien caractérisée.

Les premières exacerbations ne perdent pas ordinairement le caractère qui est propre aux fièvrès d'accès, elles débutent par un frisson, mais court et avec tremblement; une chaleur forte et longue lui succède, et les accidens inséparables des forts accès ont coutume de l'accompagner; le relâchement survient sans sueurs, quelquefois seulement avec des moiteurs aux mains, et la rémission commence.

Mais bientôt le type des exacerbations est plus confus. Le frisson disparoît de leurs premiers périodes, pour être remplacé par le refroidissement des extrémités et du bout du nez, par des fusées de froid qui partent de l'épine 
du dos pour se répandre subitement dans les membres, et pendant lesquelles on trouve des concentrations alternatives du pouls, quelque fois par une toux aiguë, sèche. Le second période est accompagné de symptômes plus accạblans, et à peine une légère souplesse de la peau marque la chûte du paroxisme.

Cependant les remèdes appropriés parviennent à modérer l'orage. Le pouls est mou, fans être plein et un peu fréquent; les sueurs s'établissent, le ventre s'ouvre, les urines se chargent, et la cauluse matérielle qui sort par tant d'émonctoires, ne laisse aucune trace de son infection. Les malades n'ont point à traîner, pour convalescence, les fâcheuses alternatives d'une fièvre d'accès. Au contraire, si le mal a une action corruptrice, les engorgemens se forment, le sang se décompose, la peau se couvre d'érúptions, et le malade meurt victime d'une fièvre très-aiguë, dont on n'a pu arrêter les funestes accroissemens.

La crise de ces fièvres se fait d'abord par des sueurs universelles, après quoi les selles et les urines prennent leur cours; car ce n'est point ici comrise dans d'autres circonstances oì les grandes évacuations par la peau, rendent les selles dures. Au contraire, les déjections alvines sont très-libres et abondantes, toujours 
dans les fièzres rémittentes.

molles, si la crise est complette; et c'est-là la marque de cette espèce de crise, et ce qui la dis" tingue de celles qui sont incomplettes ou partielles, parce que la fièvre ćtant un spasme et une constriction universelle, une crise parfaite est aussi un relâchement universel et une ouverture qui se fait par toutes les secrétions et excrétions; au lieu qu'une crise partielle n'est autre chose qu'un cours libre qui s'ouvre en particulier à quelques secrétions et excrétions, les alltres restant toujours obstruées (Grant).

XXXVII. Les fièvres rémittentes de l'automne ont au contraire le plus souvent pour début, le plus haut degré des fièvres rémittentes dégénérées du printemps. C'est presque l'image d'une fièvre ardente qui tombe subitemont en quarantehuit heures, ou en trois et quatre jours. La rémittence est alors la marche la plus durable de la fière, qui finit pour l'ordinaire par des accès de fièvre intermittente plus ou moins opiniâtre.

Parmi les étrangers, certains sujets qui n'avoient jamais eu auparavant des fièvres d'accès; des enfans et le pauvre peuple qui vit avec peine; les manouvriers qui le jour travaillent aux ardeurs du soleil, qui le matin et le soir sons exposés à une humide fraîcheur, sur-tout aux

F 2 


\section{$84 \quad$ De l'usage du Quinquina}

vapeurs qu'exhale la terre (I), et qui ne peuvent ou ne savent pas se garantir de l'humidité

(i) On voit ces vapeurs tranfpirées de la terre, après le foleil couché, fous la forme d'une fumée blanche qui couvre les terres graffes à la hauteur d'environ deux pieds; on les retrouve le lendemain qui se jouent au milieu des arbres à l'heure du matin ou la réverbéra ticn des rayons du foleil les fait diftinguer du refte de l'atmofphère. Il paróit que l'artouchement de ces vapeurs dont la nature eft bitumineufe fur la furface du corps, enduit lés organes de la tranfpiration d'une fubitance qui gêne cette excrétion; de plis, par fon mêlange avec l'air qu'on refpire, \& avec les alimens, ces bitumes fubtils s'introduifent dans la poitrine \& Chimie démontre qu'ils ont une grande affinité ; ils fe combirent avec elle, l'exaltent \& occafionnent par-là de grands dérangemens dans l'économie animale. Les maladies qui réfultent de cette influence, font les fièvres bilieufes ou putrides; elles attaquent ceux qui habitent les environs des marais et des terres chargées de bitume, et dans les faifors où la terre a pleine liberté de tranfpirer, comme en automne \& au printemps. Plufieurs circonftances que l'attention permet de faifir, prouvent qu'on n'a pas trouvé à ces maladies de caufe plus naturelle que la tranfpiration des terres; en effet, elles n'attaquent prefque jamais qué les pauvres gens \& les gens de la canpagne; elles font communes parmi les foldats campés, \& menacent tous ceux qui vivent de beaucoup d'herbages, dans les faifons que je viens de citer. Les habitans des villes dont le fol eft couiert 
dans les fières rémittentes.

de la nuit; ceux qui boivent de mauvaises eaux ou des liqueurs, vappides; ceux qui mangent beaucoup de légumes (I), des fruits passés, et même des poissons mollasses, tombent dians la langueur, perdent l'appetit, deviennent de plus en plus indolens, et après s'être plaints de l'estomac, des reins, de tous les membres, sont saisis d'un froid plus grand et généralement plus long que dans la plûpart des autres fièvres. La chaleur qui suit, devient très-ardente, et donne tous les accidens qui lui sont propres; elle est néanmoins sujette à̀ des variations irrégulières, eile tombe enfin au bout de 30 ou 36 heures, après avoir souvent redoublé au moment quili sembloit qu'on alloit suer; mais la rémittence est encore obscure et fort incertaine, quelques frissons se font sentir à l'instant où les moiteurs menacent de se déclarer; d'autres fois le ventre

de pierres, n'en font jamais incommodés, fans que le Médecin re puiffe trouver dans leur régime quel. qu'erreur relative à la trabfpiration, comme celle de s'êrsexpofé aux vapeurs qu'elle exhale après le coucher du foleil, ou d'avoir mangé des herbes couvertes de ces vapeurs.

(I) M. Retz, auteur de la note précédente, s'elt convaincu par l'obfervation que les maladies bilieufes dérivent très-fouvent de la confommation des herbages impregnés des vapeurs tranfpirées de la terre. Misiorol.

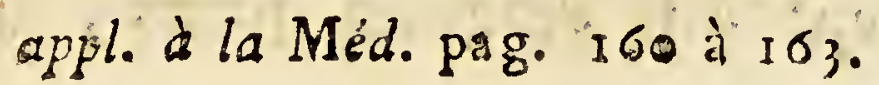


souvrira, ou les urines deviendront abondantes, variables, tantôt pâles et crues, tantôt crues et fort colorées, restant pâles ou devenant troubles en se refroidissant; pendant ce temps-là, le pouls est irrégulier, quelquefois plein et fort, quelquefois petit et dur, quelquefois assez mou, mais toujours plus fréquent qu'il ne devroit être. Les douleurs qui existent, changent souvent de place, et sont rarement fixes; enîn, l'irrégularité dans la fièvre et dans les symptômes, est très-grande, et c'est cette irrégularité qui caractérise le mieux le fond de la maladie, et sa tendance à devenir intermittente.

La nature s'étant débarrassée d'une partie des sucs nuisibles par les évacuations qu'elle suscite elle-même, ou étant secourue par celles que lart sollicite, la fièvre prend, au troisième ou quatrième jour, une marche régulière; les paroxismes en tiserce ou double tierce, quelquefois en quarte, sont très-bien marqués, et les rémissions sensibles, quand la maladie est simple er bénigne. Après les premières crises, les exacerbations débutent par un frisson apparent, et finissent par des urines qui dépofent un sédiment de couleur de brique pilé, ou par des selles fétides et crues, quoique partiellement critiques. Enfin, au bout de quatorze, dix-sept ou vingtun jours, la 


\section{dans les fiévres rémittentes}

maladie est parfaitement jugée, ou si elle ne l'est pas, la rémission disparoit, et la fièrre est véritablement intermittente, tierce ou quarte, selon la constitution épidémique, ou les circonstances particulières à l'individu affecté.

Si la constitution de l'air qui produit ces sortes de fièvres, est pour ainsi dire renforcée, elles deviennent, et beaucoup plus communes et plus fâcheuses, car on trouve presque toujours tine intime relation entre l'activité de la cause ct l'extension de l'effet. Les exacerbations qui pour lors sont accompagnées, soit d'assoupissement, soit d'anxiétés, de cardialgie, de cholera morbus, ou d'autres symptômes ( $\$$. XI, XII, XIII.) effrayans et plein de danger, présentent ces sortes de fivvres sous l'aspect des maladies graves. Tantôt elles n'en ont quie l'apparence, par la facilité qu'elles ont à céder à un traitement méthodique, tantôt elles le sont réellement par les complications qui les rendent mortelles. En effet, ces fièvres tournent quelquefois en mal, ou sont originairement d'un mauvais caractère et ' malignes, soit à cause de la température très-corruptive de la saison, soit par des circonstances attachées au malade même, soit enfin à raison d'un traitement inconsidéré : pour lors, la rémission disparoît par la durće des reprises qui se rapprochent insensiblement au 
point de se réunir ; les symptômes fâcheux qui ne se montroient quie pendant les paroxismes, diminuent tout au plus, ou conservent la même intensité pendant le temps qui devoit être occupé par la rémission, et la maladie devient inflammatoire, putride ou maligne continue (I), conséquemment d'in événement on né peut plus douteux. Mais le caractère de ces fièvres n'en esi pas pour cela dénaturé, car lorsqu'elles doivent prendre une bonne tournure, la continuité disparoit, les rémissions deviennent encore trèsmarquées, et à force de s'éloigner, de s'affoiblir

(I) De pareilles tranfmigrations \& la promptiture avec laquelle une fière rémittente paffe à la continuité, doivent fans doute être confidérées comme la fource des erreurs \& de la difpute des Auteurs au fujet du caractère des maladies. Un lecteur attẹntif ne trouvera guère de différence entre les expofés de Lind, Roupe, Pringle 8x Huxam concernant la fièvre rếmittente maligne, \& la defcription que Monro, Pringle 8x. Junker donnent du typhus, L'origine, les progrès, les fymptônes \& la terminaifon, font à peu prẹ̀s les mêmes; la feule chofe qu'on remarque, eft que, dans le commencement, la rémiffion cft plus ou moins confidérablc. La maladie décrite par Junker \& Ruland, laquelle régna dans l'armée en Hongrie, étoít rémittente dans fon principe, \& devint enfuite continue fans changer de type en aucun temps. Dans les progrès des autres maladies épidémiques, il fe fait quelquefois un changement parcil, quoique l'effence de la maladie refte la mème. 
dans les fièvres rémittentes.

insensiblement, les exacerbations ne sont plus que des accès de fièvre intermittente, qui durent pendant toute la convalescence et quelquefois pendant beaucoup plus de temps encore.

XXXVIII. Les fievres rémittentes d'automne dégénérées d'intermittentes, ayant un début analogue à celui de quelques autres fièvres continues, nous nous sentons comme entrainés à insister sur les indices qui les caractérisent. Le diagnostic est, comme on le sait, la partie la plus essentielle en pratique; ct, comme l'a dit Hippocrate, on sera toujours en état de guérir, lorsqu'on aura été à même de bien saisis le caractère de la maladie.

Or, pour ne pas s'en laisser imposer par linvasion orageuse des fièvres automnales, on s'attachera aux circonsiances que nous allons décrire. Ces maladies règnent principalement dans une saison où les chaleurs du jour et les fraîcheurs de la nuit forment une température qui réunit presque deux extrêmes; le temps est calme et l'air chargé d'humidité (I); les eaux des marres,

(1) L'bumidité dont nous parlons n'eși pas celle qui est procurée par les pluies. L'hygromètre, parfaitement gradué, nous apprend que cette humidité est beaucoup plus forte dans les temps couverts de longue durée, dans les temps couverts qui précèdent lẹs pluies ou qui succedenr aux grandes pluies; le vent du midi, qui soufle 
des cloaqué, des marais sont très-basses, et le sol (I), en plusieurs cndroits, n'est recouvert que d'eaux bourbeuses; en d'autres endroits, la tourbe est à sec, et les vapeurs qui s'en élèvent sont très-abondantes.

Si l'épidémie ne commence de très-bonne heure, la fièvre rémittente ne reste pas longtemps à se former, c'est-à-dire, à prendre la marche qui lui est propre. Les fièvres intermittentes sont en assez bon nombre, et dénotent la constitution épidémique.

long-temps; rend encore l'air fort humide; et M. le Roi a démontré que la suspension de l'eau dans l'atmosphère, et son point de saturation étoit relative au degré de chaleur. On sait que plus l'air est humide, plus il se charge des miasmes qui exhalent de tous les corps.

(I) Dans une ville voisine, St. Gilles, que j'ai habitée pendant quelques années, des raisons économiques permettent aux habitans de répandre dans les rues, pendant les deux premiers mois d'auromne, de la paille, des roseaux, \&c., pour en faire du funier. A cette époque les fièvres intermittentes et rémittentes règnent avec fureur ou redoublent. Je fus attaqué d'une fièvre rémittente qui commença le premier septembre du premier automne que je passai dans cette ville. M. de S...... mon contrère, qui m'a succédé dans certe ville, a eu le même sort, \& m'a dit que, depuis cette époque, il connoît, à un mal de zête très-forr, le jour qu'on commence à faire du fumier dans les rues. 
dans lès fièvres rémittentes.

Mais les fièvres rémittentes débutent presque toujours à l'instar d'une fièvre ardente; le frisson est fort et long, après quoi la chaleur et la fièvre sont considérables. L'urine qui a été trèsclaire et rouge, dès la première rémission, dépose un sédiment copieux de couleur rousse, ce qui est un signe, suivant Harvey (I), que le paroxisme a été fort long, oragetix et d'une terminaison difficile. Cette première exacerbation est suivie de plusieurs autres reprises irrégulières, venant au nombre de deux, quelquefois même ait nombre de trois en vingt-quatre heures, pendant lesquelles l'urine varie on ne peut davantage. La couleur dominante de cet excrément est très-haute, il y a un nuage d'un tissu trèslâche, pour la plupart du temps brun ou rou* geấtre, et s'étendant depuis la superficie jusqu'au milieu ou au fond du vase. Dès que les rémissions deviennent bien distinctes et régulières, l'urine est fort saturée et laisse échapper un sédiment de couleur de brique pilée mêlée d'une terre blanchâtre : signe constant de la cause bilieuse de la maladie et dela destruction presque générale de l'érétisme (2). Enfin les exacerbations prennent un ordre typi-

(1) Ars fanandi per expectat. T. I, p. 2 I 2.

(2) Voy. le mém. de M. Hallé, surr l'urine, dans le troisiéme yol. des mémo de la Soc. Roy. p. sio. 
$92 \quad$ De l'usage du Quinquina

que, que la fière soit plus ou moins bénigne: L'intensité des symptômes en fait toute la différence.

XXXIX. En résumant tous nos détails $\{\$ X X X V$ à XXXVII), nous voyons $\mathrm{I}^{\circ}$. que les fievres intermittentes du printemps, lorsqu'elles étoient telles dès le commencement, dégénèrent fréquemment en rémittentes, à proportion que le temps devient plus chaud, ct que les fièvres continues d'automne, qui passent très-vîte au caractère rémittent, se décomposent en intermittentes à mesure que le temps se rafraîhit et se tempère. Il est même d'observation, que, si dans le printemps, lorsqu'il ne règne encore que des fièvres d'accès, il survient subitement une chaleur humide, les fièvres intermittentes se changent toutà-coup en rémittentes même en continues; tandis que, dans lautomne, sil se fait subitement un froid mordant, les fièvres continues ou rémittentes qui régnoient pour lors, passent au caractère intermittent ou prennent celui de la constitution épidémique. $2^{\circ}$. Que les fièvres intermittentes du printemps ne peuvent point être traitées par des remèdes chauds, incendiaires; sans courir les risques de les faire dégénérer en rémittentes et en continues, tandis que les fièvres rémittentes d'automne n'admettroient pas une méthode tempérante et froide trop prolongée, 
dans les fièvres rémittentes.

sans qu'il ne vînt à leurs suites des fièvres d'accès très-opiniâtres. L'expérience a de nouveau parlé sur ce point. Elle a constaté que, sagement dirigée, la fievre bilieuse d'automne prend bientôt la marche rémittente pour devenir ensuite fièvre intermittente régulière, qui se guérit facilement lorsque le malade n'a point été affoibli outre mesure par des remèdes déplacés ou devenus inutiles; mais qu'au printemps la gradation se fait en sens inverse, lorsque les médicamens ne sont pas appropriés; car alors une fievre tierce régulière devient quotidienne, ensuite rémittente, delà ardente et enfin putride, sur-tout si l'on a insisté sur une méthode chaude et trop active.

$3^{\circ}$. Qu'il faut considérer, dans les fièvres printannières, une disposition inflammatoire du sang, et conséquemment un génie phlogistique $(\$ \mathrm{XXX})$; dans les fièvres automnales, une disposition humorale (I) et conséquemment un génie bilieux, ( $\$$ XXXII) ou putride ( $\$$ XXXI); car l'état de nos

(I) C'eft ce que Haartman a voulu exprimer, en disant que les fièvres printannières proviennent d'une trop grande abondance de sang, comme celles de l'automne sone dues à l'excès de sérosité. A ces causes se joignent encore au printemps, les changemens de l'air, favorables à la vigueur du corps; et en automne ceux qui ôtent les forces ( 2 vol. des nouv. mém. de l'Aczd. Roy. des sciences de Stockolm, art. s. 


\section{De l'usage du Quinquina}

fluides change suivant l'influence des saisons; et la nature de nos maladies, quel que soit leur type, répond ou participe exactement de la qualité de nos fluides. Nous ne chercherons pas loin les preuves de cet énoncé. Dans le printemps, l'air est serein, le temps est vif, le sang est riche et la réaction des vaisseaux est forte; la saison qui a précédé a favorisé la congestion des sucs dans le tissu cellulaire, mais ces sucs ne sont pas âcres, ils ne sont que grossiers; le temps de leur atténuation, de leur expulsion successive est venu. Quelquefois les mouvemens, que la nature emploie à cet ouvrage, sont trop tumultueux, et ils forment une fièvre d'accès ou une fièvre rémittente ; mais cette fièvre doit nécessairement tendre à l'inflammation: l'état du corps, avant linvasion de la maladie, en est la preuve la plus démonstrative. Au contraire, dans l'automne, l'air est humide et mal-sain, le temps est mou, le sang est appauvri, et les fibres affoiblies réagissent plutốt par leur mobilité morbifique, que par leur force intrinsèque; la saison qui a précédé, a dépouillé les humeurs d'une aquosité nécessaire; les fluides sont plus épais et leur consistance est tenace jusqu'à un certain point; ils sont âcres et ont une pente à la dégénération bilieuse ( $r$ ); la fièvre qui survient dans cet état,

(1) Voy. Grant, Rech. fur les fièvres, qui a dit, à cet égard, des choses fort judiciouses. 
dans les fièvres rẻmittentes. ne peut être qu'informe. Aussi la première exacerbation est longue et forte, parce que le spasme universel appeié rigueur a été violent et prolongé. Mais on ne le sent que trop, cette intensité de la fièvre étoit utile; c'est à elle que sont dues les évacuations critiques partielles qui amènent la rémittence, parce qu'il est de fait que les humeurs sont d'autant plus atténuées, que l'action des artères a été vive pendant le feu fébrile. Cependant les mêmes causes qui ont produit la première exacerbation continuent, et l'effet en est semblable au précédent : la maladie est seulement moins forte et plus régulière par rapport aux crises incomplettes qui ont eu lieu. La fièvre revient et est suivie d'une autre coction et d'une autre crise, de sorte que le second paroxisme semble être plutôt une seconde fièvre qui ne provient point de la première, mais de causes semblables, et il lui ressemble en apparence. Enfin ces coctions et ces crises partielles mais réitérées font cesser la rémission; et la fièvre reste intermittenté jusqu'à la coction et la crise générale et parfaite:

XL. Telles sont les nuances qui distinguent et qu'on trouve dans les fièvres du printemps ( $\$ X X X V I)$ et dans celles d'automne ( $\$$ XXXVII) prises abstractivement des complications fortuites qui dépendent d'une intempérie de la saison, des 
96

De l'usage du Quinquina

circonstances individuelles et de quelques autres causes accidentelles et passagères, ou de l'influence de l'épidémie régnante, dont la fièvre rémittente forme la maladie intercurrente. Mais comme ce sont moins les saisons que les intempéries qui dominent dans ces saisons, qu'il faut considérer pour voir ce que sont les maladies, on ne doit pas oublier que, l'hiver ayant été humide et chaud, privé de gelées, de venis du nord, la constitution du printemps est vraiment autom. nale; tandis que l'été n'ayant pas été légitims aussi, c'est-à-dire, qu'ayant été sec et frais, raffraichi par des vents septentrionaux, la constitution de l'automne est véritablement printannière. Observons que, dans ces constitutions déplacées, la marche des maladies doit souvent tenir de celle qui est propre aux fievres du printemps et à celles d'automne; on en sent la raison, et nous le prouverons par un exemple.

XLI. Les habitans d'une ville voisine. (Lunel) et de la plupart des lieux circonvoisins, essuyèrent en $I_{7} 80(1)$ une épidémie très-générale ( $\left.\$ X I I I\right)$,

(1) Cette épidémie ne régna qu'en 1781 : nous en changeâmes la date pour éviter cette manière indirecte de nous faire connoître, ayant décrit cette épidémie que nous avons observée dans ui mémoire adreffé à la Sociétć Royale de Médecine, qui l'a couronné par un prix d'encouragement. (Note ajoutée.)

elle 
dans les fièvres rémittentés.

elle débuta à-peu-près vers l'équinoxe du prin-: remps, sous une intempérie automnale, et son caractère conserva quelque chose de l'influence. du printemps et de l'automne.

La fièvre étoit rémittente et commençoit par quelques accèsde fièvre tierce, qui se changeoient. en double tierce, et qui, plis ou moins promptement, perdoient le calme de l'intermission. Après les remèdes appropriés, la fièvre reprencit sa première marche intermittente, et conservoit ains le caractère des fièvres printannières et des automnales.

Les urines ne déposoient pas de sédiment briqueté ; cependant la fièvre étoit de l'espèce des intermittentes, dégénérée en rémittente, rare-i ment en vraie continue, Les frissons qui commençoient chaque exacerbation de la fièvre rémittente, les sueurs ou les moiteurs qui la terminoient, le début et la décomposition de la maladie en fièvre intermittente, les effets du. quinquina ( $\$$ XLIX), en étoient la preuve 1 a moins équivoque.

Si l'on n'avoit considéré que Pintensité ou l'urgence de certains épiphénomènes, tels que le cholera-morbus, qui étoit un symptôme trèscomnun, des romissemens opiniâtres et laborieux, des déjections séreuses ct très-âcres, des coliques eruelles, une expectoration pénible 
bilieuse ou sanguinolente, l'oppression, une toux douloureuse : lorsque la fièvre portoit sur la poitrine, le délire, l'assoupissement, de petites hémorragies dé nez: quand la tête étoit prise, les soubresauts des tendons, le pouls serré, des ressautemens convulsifs, lorsque l'âcre bilieuse affectoit de préférence le système nerveux et vasculaire; si, disons-nous, on n'avoit considéré que la violence de ces épiphénomènes, on n'auroit pas manqué de s'attendre aux évé. nemens les plus fâcheux, on auroit été tenté de ranger l'épidémie dans la classe des malignes. Ces symptômes n'étoient cependant qu'effrayans, ils paroissoient dans le paroxisme, et ne laissoient aucune trace pendant la rémission, pourvu que les indications ne fussent pas méconnues.

On justifia ce pronostic sur un très-grand. nombre de malades. Quelques-uns seulement payèrent tribut à la nature, et la fièvre chez eux ne fut maligne qu'en raison des circonstances. En général cependant la convalescence étoit délicate, les rechûtes étoient aisées, et si l’o ne se conduisoit pas prudemment, on n'étoit délivré, qu'au retour de la belle saison, de la fièvre d'accès consécutive.

Une observation, que des faits rëitérés rendirent concluante, c'est que, chez le plus grand nombre de malades, ceux qui furẹnt attaqués 
dans les fièvres rémitterites.

la lièvre rémittente en automne, éprouvèrent au printemps d'après une fièvre tiémittente trèssimple ou une fièvre d'accès béniggtne, tout comme ceux qui furent pris au printemp̣s de la fièvre rén mittente, essuyèrent, l'automrie suivante, une fièvre intermittente ou rémittente, qui cèdoit facilement à une bonne méthode curative.

XLII. Nous avons en quelquefois occasion de le remarquer; les fièvres rémittentes s'éloignent d'autant plus du caractiere que leur imprime la saison ou l'épidémie; la marche de ces fièvres; leur type, leur génie se dénaturent d'autant plus; qu'il se réunit, dans les sujjets affectés, des circonstances tout-à-fäit étrangères à la maladie, et qui, lui étant antérieures, forment des coms. plications ( $\$$ XIV ) plus ou moins dangereuses:

Par complication, il faut entendre avec nous; soit certains vices de constitution, tels qu'un excès d'irritabilité ou d'atonie, soit l'affoiblissement relatif de quelque organe, comme du cerveau, du poumon, du mésentère, soit un principe morbifique inhérent aux solides, tels que trop de rigidité, trop de délicatesse, ou tenant aux fluides, tels qu'un virus dartreux, goutteux, psorique, vénérien, scorbutique (I),

(1) En plaçant le scorbut parmi les maladies qui sont dues d'une manière particulièrè la dépravation du fuide vital, nous ne prétendons pas prononcer entre cette ogit,

G 2 
soit certaines lésions constitutives d'une affectio chronique, lorsque, par exemple, il y a des obstructions, des ulcérations dans quelques viscères, soit enfin une maladie aiguë, cəincidente, mais majeure, telle qu'une fièvre stercorale vermineuse, etc.

Et comment ces complications n'influeroientelles pas pernicieusement sur le caractère des fièvres rémittentes, purisque, dans ce cas, une maladie plus ou moins grave, est hantée sur une autre maladie qui présente plus ou moins de danger? L'observation n'en a que trop souvent convaincu. Les fièvres ainsi compliquées offrent non seulement des épiphénomenes plus effrayans et plus fâcheux, mais encore elles sont susceptibles d'une dégénération plus rapide, elles exigent plus de sagacité dans l'observateur, elles annoncent plus d'incertitude dans l'événement, et font craindre plus d'inconvéniens pour les suites.

XLIII. Eh certes! si l'irritabilité est la faculté inhérente à la fibre animale, de se contracter

nion et celle qui admet, pour cause du scorbut; le défordre primordial des folides (Voy. M. Milman*), mous voulons feulement nous conformer au fentiment xeçu.

* An inquiery in to the fource from Whence the simptoms of the fcurvy and of putrid fevers arise, E.6. I7 $8 \mathrm{k}$. 
dans les fièvres rémíttentes:

après avoir été stimulée par un irritant quelconque, l'excès de cette irritabilité, sans constituer proprement ce qu'on appelle une maladie, doit cependant en approcher de si près, çu'il suffit, avec cette condition, d'une cause très-légère, pour qu'il s'établisse des lésions morbifiques. L'excès d'irritabilité est peut-être le premier. degré du spasme. Et à combien de maux ce spasme augmenté ne donne-t-il pas naissance? L'inflammation locale en est un des plus réels et des plus communs, et la violence des symptômes étant proportionnée à l'excessive irritabilité des vaisseaux, il en résulte promptement la gangrène, et la destruction des organes quii étoient le siège du mal. Mais quels sont les cas où l'on trouve ordinairement cet excès d'irritabilité ? On sait par expérience que ce vice est presqu'inséparable des personnes qui ont les fibres très-minces et très-délicates, conséquenment très-mobiles; et tel est un grand nombre de femmes, d'enfans. On le trouve dans les personnes dont le corps, quoique robuste, est sec, maigre, chand, avec des muscles fermes et rigides, qui ont de lactivité et de la promptittide dans les actions. Il existe encore dans totis ceux qui souffrent d'une irritation locale et permanente; tels sont les enfans pendant la dentition, dans le temps de leur crue, pendant le dé

G3 


\section{'ro2 De l'usage du Quinquina}

veloppement de la puberté; telles sont les femmes grosses; tels sont encore la plupart des mélancoliques et des hypocondriaques. Ajoutans que l'excès d'irritabilité est propre aux habitans des pays chauds, et par analogie que cet excès se développe dans la plupart des individus qui vivent sous un ciel tempéré, mais qui souffrent accidentellement de l'inclémence d'une saison sèche et brûlante.

XLIV. Une grạnde atonie forme l'état opposé, et présente une perspective plus affligeante encore. Ici le sang est̃ peu animalisé, et l'inertie des fibres suppose une très-grande activité dans ie principe morbifique, comme elle annonce beaucoup de lenteur dans la coction et presque la certitude d'une maladie cachectique consécutive. Sil se forme des engorgemens très à craindie dans ces circonstances, en raison de l'inégalití. d'atonie, la gangrène est un accident à redolter, parce qu'ayant précédé une action violente des fibres motrices, pendant le feu fébrile, l'atonie qui suit cette action devient plus complette et conséquemment peut être la cause prochaine de la gangrène; car les vaisseaux n'agissant plus sur les fluides quils contiennent, ces fluides cessent de circuler et se corrompent très-promprement. Cet excès d'atonie est remarquable chez les gens incapables de travanx soutenus, done 
dans les fièvres rémittentes.

Je teint est pâle, les urines décolorées, les déjections glaireuses, chez ceux qui sont sujets. aux bouffissures; il est spécialement affecté alıx fièvres qui se déclarent, après une constitution long-temps humide, nébuleuse de l'atmosphìre, laquelle a singulièrement relâché le tissu des fibres. Il existe ordinairement après des maladies. séreuses, après de grandes évacuations. On le trouve enfin chez les habitans des contrées froides et humides, chez les hommes que la profession expose à être continuellement sur l'eat, comme les pêcheurs, et en général chez les gens mal-aisés, chez les hommes d'une classe fort pauvre et fort laborieuse.

XLV. Il est un état mixte, composé, pour. ainsi dire, de trop d'irritabilité et de trop d'atonie, lequel réunit, jusqu'à un certain point, les inconvéniens attachés à ces deux extrêmes. M. Fouquet appelle cet état, laxité vibratile, parce qu'il faut caractériser un tempérament spasmodique et débile qu'on observe chez quelques petites filles de neuf ou dix ans, maigres, vives et d'une taille svelte, en général sur les. enfans précoces ou d'un esprit prématuré (I).

XLVI. Quand la force d'un organe est audessous de celle qui lui est nécessaire pour main-

(r) Traité de la petite vérole. T. I, p. I8I.

$\mathrm{G} 4$ 
tenir l'équilibre du systême général des forcés. organiques, il est indubitable que l'organe affoi*

- bli ne forme un centre, oì convergent, pour ainsi dire, tous les mouvemens qui s'opèrent dans l'économie animale. Dès-lors s'établissent Ies complications locales qui peuvent d'autant plus aisément dominer dès le commencement même de la fièrre, que la partie débile a souffert des aiteintes plus considérables de la part des causes qui ont déterminé la maladie. Que cette plus grande foiblesse relative d'un organe, soit l'effet d'un vice héréditaire', inné ou acquis, qu'elle soit l'efét de l'influence épidémique, il est certain qu'elle prête spécialement aux lésions primitives que procurent le niasme épidémique ou toute autre cause nuisible; et pour lors les symptômes sont, dès leur naissance', d'une gravité très-disproportionnée à l'état d'activité générale des forces, qui a immédiatement précédé. N'y a-t-il pas lieu de croire que les affections comateuses, que les symptômes pleuropéripneumoniques, etc., qui compliquent les fières rémittentes dès leur début, n'arrivent qu'en vertu de l'extrême disposition que le cerveau, la poitrine, etc., relativement plus foibles, offrent au dépôt de la cause matérielle de la fièvre Et n'est-il pas naturel de présumer que, puisque le mésentère est si souvent le siège des affections. 

ordinairement graves et dangereuses, chez tous ceux qui abusent habituellement de la bonne chère, cet organe doit être, chez ces sujets, la partie la plus foible, et par conséquent une partie très disposée aux lésions fortes et dominantes?. Aussi voyons-nous que des auteurs (Hoffmann, Spigel, Baglivi, ) très-recommandables, ont cru que la fièvre hémitritée, une des plus malignes des fièvres rémittentes, étoit uniquement occasionnée par l'inflammation du mésentère.

XLVII. Dans les maladies, les efforts de la nature sont toujours proportionnés à l'activité du principe morbifique, toutes les fois que les forcès du corps sont en raison de l'intégrité qui constitue l'état sain et naturel. Mais n'arrive-t-il pas souvent qu'une cause légère excite des mouvemens violens? N'a-t-on pas souvent lieu de remarquer que les efforts de la nature, loin d'être relatifs à la cause excitante, ne font que répondre à la manière de sentir d'un être infirme; et que les forces, trop énergiquement excitées, agissent en raison composée, soit du principe morbifique, soit de l'extrême sensibilité du malade (I)? De pareils effets ont inévitablement lieu lorsque les fibres pèchent par trop de rigi-

1I) Voy. Lorry de Morborum mutatianibus, p. I z̧. 
106 De l'usage du Quinquina

dité, ou par trop de délicatesse. Des fibres. trop délicates, telles que les ont ces sujets, qu'affoiblissent la vie sédentaire et la mollesse, oscillent tumultueusement, et secondent, avec une incroyable activité, les lésions que le principe morbifique opère. Des fibres trop roides, telles que les ont ces hommes que dessèche un feu violent auquel certaines professions exposent ou que la fatigue endurcit, opposent à l'acrion du mal une résistance d'autant plus dangereuse, que venant à s'affecter, elles allument une fièvre plus vive, et cntretiennent penciant plus long-temps sa formidable incandescence. De ces deux chefs primitifs dérivent d'autres accidens secondaires. $I^{\circ}$. Dans une constitution qui pèche radicalement par la délicatesse des fibres, l'ardeur fébrile crée pour l'ordinaire, d'une part, une plus grande masse d'humeurs excrémentitielles, ce qui fait que la coction peut rarement être parfaite; de l'autre, elle porte le spasme à un trop haut degré, pour qu'il ne s'établisse pas des centres inflammatoires, elle force trop rudement les vaisseaux, pour ne pas faire rompre ceux qui en souffrent davantage. Aussi voit-on survenir alors les délires frénétiques, les oppressions douloureuses, le météorisme inflammatoire, les douleurs vives du foie, les hémorragies du nez, les pertes utérines, etc. 
épiphénomènes effrayans autant que périlleux, et qui, sans le vice préexistant des solides et des fluides, n'acquerroient jamais aussi promptement le degré de violence qui les caractérise, et ne persisteroient pas d'une manière aussi duable, $2^{\circ}$. Dans une constitution qui pèche foncièrement par trop de rigidité dans les fibres, cette même ardeur fébrile toujours portée au comble, non seulement suspend, dans peu, toutes les excrétions et rend extrêmement pénible l'exercice de toutes les fonctions, mais encore elle entraine à sa suite tous les maux occasionnés par un excès d'atonie ( $\$$ XLIV) ; car cet excès d'atonie vient ordinairement après un excès de tension et d'érétisme, comme la dissolution putride vient au plus haut degré après la densité inflammatoire ( $\$ X X X I)$; et voilà ce qui rend les maladies putrides, plus meurtrières pour les paysans que pour les hommes d'une condition aisée; voila ce qui rend si difficile, dans les constitutions de la fibre roide, les convalescences des maladies caractérisées par les symptômes d'une violente ardeur: tels sont les yeux brillans, une forte dyspnée, la constipation, la paucité des urines âcres et enflammées, la chaleur de l'halcine, la vivacité de la soif, l'ictère, etc. Faisons observer jci, au sujet de la jaunisse, que cet accident peut dépendre ou de l'épaississement résineux 
108

De l'usage du Quinquina

de la bile, et.pour lors les malades sont cons:tipés, soufirent d'ardeurs intolérables, et tombent presqu'inévitablement dans la cachexie; ou de l'incandescence ou de l'extrêne âcreté de cette lique:ir, qui, par ses impressions cruelles, multiplie les points d'inflammation, et, par ces irruptions violentes et soudaines, occasionne des cholera-morbus et des dyssenteries.

XLVIII. Ainsi se forment les complications par un vice préexistant des solides. A-t-on moins à craindre 'ie celui des humeurs? Non, sans doute, et l'on a tout à redouter des maladies virulentes clont le principe est d'une grande mobilité, et capable de se fixer sur les parties internes. En cffet, lorsque la fièvre surprend les personnes attaquées de ces maux, elles en ressentent des atteintes plus vives dans le paroxisme qui les renouvelle même souvent, quoiqu'avant la fièvre il n'y en eût aucun symptôme, et alors il est à appréhender que l'ancienne matière morbifique mise en mouvement et aigrie par la fièvre, ne se porte sur des viscères essentiels; il en résulte encore quie les solides et les fluides étant aitérés par deux causes à-la-fois, il s'établit des lésions graves et difficiles à détruire. Par exemple, l'humeur goutteuse étant mise en mouvement par la cause matérielle de la fièvre, les. malades éprouvent, dans le paroxisme, de vraies 
douleurs de gout1e dans les jointures, ou ils ont du moîns des signes manifestes de goutte; il en reste un ressentiment après qu'il est fini, ce qui, suivant M. Colombier ( $\mathrm{I}$ ), distingue ce cas de celui oủ les mêmes douleurs sont simulées.

XLIX. Dans le scorbut (2) compliqué avec la fièvre rémittente, il y a moins de danger que la matière morbifique, c'est-à-dire l'humeur scorbutique, ne se fixe sur les viscères, par l'effet du levain fébrile, quoique cela arrive quelquefois; mais on a beaucoup d'autres fâcheux accidens à envisager, dont la plupart sont même inévitables. De ce nombre sont l'érosion et l'hémorragie, la syncope, qui doivent naître dans cet état par le mouvement accéléré des liqueurs à chaque paroxisme. Or, on sait combien ces accidens sont funestes dans le scorbut. On ne doit pas moins s'attendre à une dégénération

(I) Med. milit. T. I, P. 438.

(2) Lind a dit, dans fon traité du fcorbut, que cette maladie, de fa nature, eft oppofée à la fièvre, au point qu'un fcorbutique demeurera long-temps expofé à la coniagion fiérreufe fans en être infeçé; et cet auteur l'a tépété dans fon mémoire fur les fièvres. Il faut croire que tous les obfervateurs n'ont pas vu comme lui. Lorry avance, de morb. mutat. p. I 40 , que le fcorbut eft, de toutes les maladies virulentes, celle qui fe complique le plus facilement avec les maladies aiguës. 
$\$ 10$

De l'usage du Quinquina

plus prompte des humeurs par la continuite de la fièvre, et conséquemment à une dissolution plus manifeste, qui est le terme de cette nnaladie dont on n'arrête pas les progrès. Il est d'expérience que l'hémiplégie ou une diarrhée colliquative arrivent encore assez communément dans les fièvres compliquées d'une virulence scorbutique.

L. Nous faisons entre la véritable diathèse scorbutique et cette discrasie humorale dont il semble que Rivière ait parlé le premier, et que l'on connoît sous le nom de cachexie scorbutique, la différence que ces deux états méritent. Cette cachexie, très-familière parmi les gens qui jouissent abondamment de toutes les commodités de là vie, n'est autre chose qu'un certain degré d'acrimonie avec plus ou moins d'aptitude à la dissolution. Dans cet état des humeurs, les effets de la fièvre sont souvent trè̀sfâcheux par la fonte des liquides que chaque redoublement occasionne. La pletthore graissense expose aux mêmes accidens. La graisse est, dans le fond, une huile légère qui tient infiniment de la nature alimentaire et sur-tout de la matière que nous tirons des végétaux. Elle dégénère facilement, et sa dégénération est très-âcre. Aussi voit-on que chaque reprise, dont le second temps a pour l'ordinaire beaucoup d'intensité, produit 
'des urines huileuses, des selles colliquatives; les petechies surviennent, et le malade maigrit rapidement : indice non équivoque de l'existence d'un levain putride et du danger pressant où jette l'ardeur de la fièvre.

LI. M. Colombier remarque que la repercussion ou la rentrée des dartres cause ordinairement des rémittentes putrides, ou d'autres maladies plus graves ( $\mathrm{I}$ ). On conçoit donc que l'humeur herpétique peut former une fâcheuse complication. Nous en dirons autant de l'humeur psorique et du virus vénérien; observant avec $\mathrm{M}$. Lorry (2), au sujet de ce dernier, que les malades qui en sont infectés présentent plus de disposition aux maladies épidémiques qu'à toutes celles qui peuvent survenir accidentellement, et que la fièvre rémittente compliquée d'un vice vérolique est, on ne peut plus anomale et plus difficile à juger : l'influence d'un pareil virus étant aussi destructive dans les maladies aiguës que dans les chroniques.

(i) Nous ne penfons pas que le virus herpétique puisse procurer, par lui-même, une fièvre d'accès ou une fièvre rémittente, mais qu'il peut la déterminer, soit en affoiblissant les actions naturelles, soit en donnant J'éveil à la cause fpécifique de ces fièvres.

(2) Loc. cit. p. I37. 
LII. L'obstruction des viscères forme une nơt: velle complication de la fièvre, et l'on peut avancer, contre l'opinion assez commune, que la fièvre n'est pas aussi salutaire lorsqu'elle est hantée sur les obstructions, que quelques faits heureux ont porté trop généralement à le faire croire. Nous savons que lorsque la suppression mal-adroite d'une fièvre intermittente ou rémittente, aura occasionné des engorgemens, des obstructions, ces funestes produits ne sont quelquefois jamais mieux enlevés que par une rechûte de la fièvre. Mais combien de fois cette rechûte a-t-elle accéléré la dépravation de la constitution? Combien de fois le retour de la fièvre ne s'est-il borné qu'à procurer l'inflammation ou la dissolution putride du viscère affecté? Les exemples n'en sont pas rares; mais lis obstructions ne proviennent pas toujours de la fixation du levain fébrile sur les viscères, elles sont, dans bien des cas, primitives et déjà anciennes. L'obstruction des glandes mésentériques est familière chez les enfans, et le foie n'est que trop sujet à s'engorger à la suite d'une infinité d'accidens qui attaquent l'espèce humaine. Remarquons, en passant, que certains indices d'obstructions au foie, tels que le teint jaune, sale, la langue chargée, dépendent moins quelquefois d'un viscère obstrué, que d'une atonie 
'de l'estomac, très-propre à donner le change: Quant aux obstructions dégénérées en squirre, il peut en arriver que l'action de la fiève leur fasse prendre un caractere carcinomateux. Nous avons vu en 1783 , une Demoiselle de 2 r ans, très-vaporeuse, à qui les paroxismes d'une fièvre rémittente d'automne, donnoient des douleurs si aiguës dans une glande au sein, qu'il s'en suivoit les accidens hystériques les plus cruels et les plus formidables. On sait que dans toutes les fièvres compliquées d'obstructions, le pouls ne se développe jamais d'une manière aussi complète que dans les fièvres où le cours des liqueurs n'est arrêté par aucun obstacle.

LIII. Comme les ulcérations des viscères, dont celles du poumon arrivent plus fréquemment, sont toujours accompagnées d'une fièvre hectíque, laquelle a des exacerbations décidées par une cause connue, telles que l'exercice de la digestion, le mélange du chyle avec le sang, et linfluence de l'air nocturne (I), il n'est pas

(I) Suivant M. Morgan (animal ceconomy. prop. Is) le pouls de tous les homines, quelques fains qu'ils foient d'ailleurs, eft plus fréquent après qu'ils ont mangé; de forte que li l'artère bat 66 fois dans une minute le matin quand on eft à jeun, elle battra 34 fois après qu'on aura dîné : ce qui eft dans le rapport de 100 à I 29 ou i peu près comme to a 13. Cette friquence contimb 
trop aisé de connoître l'intervention d'ine fièvre rémittente qui vient former une redoutable complication. Les variétés qu'on observe dan la marche des phthisies pulmonaires ou autres, la différente intensité des fièvres rémittentes, l'anomalie d'une fière qui tombe sur un sujet énervé, la réciproque influence de deux maladies qui ont chacune un caractère fixe; toutes ces causes tendent à obscurcir cette complication, sans compter que toutes les fois qu'il existe une constitution dominante, les maladies qui règnent en même temps, prennent souvent la marche de la fièvre de la constitution, sans qu'il $\mathrm{y}$ ait quelquefois d'autre analogie, soit dans la cause, soit dans la méthode curative. Parmi les signes les plus propres à manifester cette complication, on distingue l'ingravescence de la fièvre hectique sans cause assignable, pendant

jufqu'à minuit, mais elle décrô̂t infenfiblement; de forte que le nombre des pulfations, une heure après le diné, eft au nombre de fois que l'artère bat à onze heures du Toir, comme $8_{4}$ à 78 à peu près. Comme fuivant les principes de Molgan, lorfque la fréquence du pouls eff da plus grande, celle du foir.eft à celle du matin dans le rapport de $8 \mathrm{a}_{7}$, il s'en fuit que la force que le pouls a le foir, eft à cele qu'il a le matin comme i I à I 10. La vîteffe du fang, de même que le diamètre des artères font aufit plus gands le foir que le matino. 
une épidémic de fièvre rémittente. Le diagnostic est beaucoup plus évident, swivant l'observation de Trnka (I), lorsqu'un sujet affigé de fièvre hectique éprouvera des exacerbations très apparentes par les trois temps qui les caractérisent, lorsque ces exacerbations auron une durée convenable et à peu près égale; qu'elles seront terminées par une sueur abondante, douce, universelle et suivie de quelque soulagement.

Les ulcères externes sont sans contredit d'une bien moindre conséquence, que ceux dont le siège est à l'interieur; mais comme ces ulcères; lorsquilils sont invétérés, annoncent le mauvais état des fludes, et que rarement l'ardeur de la fièvre manque de supprimer le pus qu'ils (les ulcères) distillent, un Praticien judicieux doit quelquefois imputer à cette complication quelques accidens qui traversent le cours d'une fièvre rémittente.

LIV. Mais de toutes les complications qui peuvent dénaturer cette fière, il n'en est pas de plus ordinaire que la fievre stercorale. Alors les premiers jours sont très-orageux, et l'on ne peut prononcer sur le véritable caractère de la maladie, parce que la violence et le nombre des

(1) Hiftoria febris heftica, S. 34, p. 147 . 


\section{6 De lusage du Quinquina:}

accidens le masquent. Point de rémissions lucides, point de reprises bien marquées, les symptômes de la fièvre stercorale sont constants, et sil y a constipation, ils sont portés au plus haut degré d'intensité. Ces symptônes, comme on le sait, consistent en nausées, vomissemens, déjections de ventre, borborygmes, langue chargée, dégoût, anéantissement, douleurs vagues, maux de tête, cardialgies, syncopes, dififculté de respirer, léthargie, pouls durou petit, ou serré, météorisme dans les entrailles, tension du bas ventre, insomnies, et tant que leur cause subsiste, on ne peut point espérer que la fièvre rémittente soit bien reconnoissable; mais après des évacuations copieuses par le haut et par le bas, la fievre stercorale étant détruite, presque dissipée, ou du moins subordonnée, la rémittente développe tout son caractère, et suit la marche que lui imprime linfluence de la saison ou de l'épidémie. Telles sont presque toutes les fièvres rémittentes des enfans, ces êtres doués d'une trés-grande irritabilité et de beaucoup de disposition aux indigestions. Aussi M. Butter (I) croyant très erronée l'opinion commune, qu attribue aux vers la cause de leur fièvre rémittente, s'est-il efforcé de prouver qu'elle ne doit

(1) A tratise on the infantile remittent fever, \&x. 
dans les fièvres rémittentes. être imputée qu’à la saburre des premières voies.

La fièvre vermineuse est une varićté de la fièvre stercorale ou de la fièvre putride. It faut en cffer aux vers, pour pouvoir pulltiler et crôtre, un certain amas d'humeurs croupissantes et corrom. pues. En rampant sur la tunique villeuse des intestins et du ventricule, ces insectes excitent un spasme qui produit une infinité d'épiphénomènes, entr'autres, des nausécs, des défaillances, des anxićtés, s'ils sont dans l'estomac, un pouls dérangé, foible, des douleurs pleurétiques, un resserrement du gozier, la suffocation, etc. Or, ces accidens n'étant pas subordonnés ì la fievre rémittente, on les observe, soit par périodes fixes, soit par intervalles fort irréguliers, tantôt durant le paroxisme, tantôt pendant la rémission de la fièvre qu'ils compliquent. Ce qui ne sert pas peu à jeter la plús éronnante confusion dans le diagnostic de l'une ct laurre maladie.

LV. Il ne manqueroit sans doute pas d'autres complications à ramener ici, si l'on devoit, disons mieux, si l'on pouvoit compléter cetre intéressante matière. Qu'il nous suffise de le faire observer, au risque de nous répéter encore, les fièvres rémittentes peuvent se compliquer, lors-a qu'elles sont sporadigues ou intercurrentes avec

$\mathrm{H}_{3}$ 


\section{8 De l'usage du Quinquina}

la maladie de la constitution; et pour lors, outre l'influence de l'épidémie, la fièvre se trouve réelJement compliquée. C'est en vertu de ces influences, de ces complications respectives, qu'on a vu les fièvres rémittentes, tantôt accompagnées ou prendre le caracière des fièvres catarrhales? fantôt sévir pendant une épidémie de peste, et se convertir en pestilentielles, tantôt, enfin, être tellement maîrisées par la conjugaison d'une maladie plus grave, que leur marche presqu'absolument dénaturée nadmetroit désormais quel'ordre propre à l'afection compliquante: ainsi, pour citer un exemple de ce dernier genre, la fameuse fièvre de hongrie étoit, suivant Pringle, un composé de la fic̀vre rémittente d'automne et de celle d'hôpital tirant sa source du camp, mais acquérant cette nature pestilentielle du mauvais air des endroits où l'on mettoit en foule les malades. M. Robertson est sans doute-parti de là pour dire ensuite, mais d'une manière trop générale, que la fièvre des prisons, celle des hôpiraux ou des vaisseaux, paroissent essentiellement les mêmes, et doivent probablement être rangées parmi les rémittentes malignes (I).

LVI. Nous nous sommes donc suffisamment

(1) Obfervations ou the jail, hofpital ; or Shyp fevers, 8. C. $1.7^{8}$;: 
dans les fières rémittentes.

expliqués sur tout ce" qui concerne les fières rémittentes. Leur caractère ( $\$$. II. à IV.) a été tracé, et l'on a vu quels en sont les indices ( $\$$. IV. à VI.) pathognomoniques; quels sont les indices ( $\$$. VII, X, XXXIII. ) propres à les spécifier. Nous nous sommes expliqués sur leurs causes (\$.XV.), sur leurs types ( $\$ . X V I$. d XXIX.),

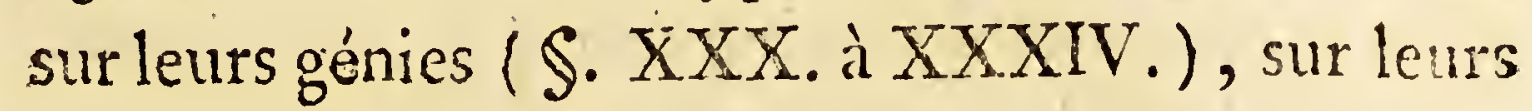
marches ( $\$$. XXXV. à XLI. ), sur leurs complications ( $\$$.XLII. a LV.), et le pronostic quion doit en tirer, dérive naturellement des circonstances exposées (\$. II. à LV.). Si la nature de ces fièvres se trouve approfondie par nos détails, ne sera-t-il pas plus aisé de prononcer sur la méthode curative? On nous demande ( $\mathfrak{S}$. I. ) de déterminer quels sont les avantages et les dangers du quinquina dans leur traitement. On prévoit, par le rapport intime ( $\$ . I I, X V, X X X V$.) que les fièvres intermittentes ont avec les rémittentes, que le quinquina employé dans la cure des premières, peut l'être dans celle des secondes et avec une égalité de succès. On sait que des Aluteurs recommandables l'ont conseillé à trèsforte dose dans le tráitement de ces fièvres, prae tique qui a souvent été utile et dont on a souvent aussi abusé. On veut qu'on recherche qu'elles sont les circonstances dans lesquelles on doit sabstenị de donner ce médicament à grandes

$\mathrm{H}_{4}$ 


\section{De lusage du Quinquina}

doses, et avec quelles précautions on peut le permettre quelquefois. Nous répondrons à toutes ces questions intéressantes, en examinant, lorsque l'occasion s'en présentera, quels sont les secours qui préparent à l'usage du quinquina; quels sont ceux qui peuvent en seconder les effets et en perpétuer les avantages?

La découverte du quinquina (I), son introduction dans les pharnacies de l'europe, ses succès, ses revers, sa proscription, son triomphe, sont des objets trop connus, trop répétés,

(I) Connu par les Indiens depuis environ I 500 , il fut apporté du Pérou ell 1640 ; répandu en 1649 ; décrit d'abord par Antoine Bollus (a) marchand Génois, \& peu après par Sebaftianus Badus en I 663. Willis commença à s'en fervir en 1657 contre les fièvres rémittenzes; Silvius Déléboë. l'imita à Leyde dès I607; Noül Falconnet a l'honneur de l'avoir employé le premier en France; 8 Reflaurand qui le fuivit, en combattit, en I680, des fièvres hémitritées. Davinius précéda en Italie 1a pratique \& les écrits de Torti; comme en Allemagne Kolzebut \& Konerding fout comptés avant Cartheufer \& Werloff. Les écrits fur le quinquina fe font depuis fort multipliés, \&, fil'on peut le dire, ils fe font accrus en raín de la diminution des exportations du quinquina, žu moins du bon quinquina. Heurcufement on en a découvert dans le nouveau Mexique, \& la Martinique peut Fournir le quinquina-piton.

(a) C'eft M. Rahn qui donne cotte priorité à Bollus. MM" Coffe Lillemet la donnent à Stum, Médeçin Greç. 
dans les fièvres rémittentes. pour que nous pensions à nous parer ici d'une érudition inutile. Ce remède a partagé le sort des découvertes : vanté pur le crédule enthousiasme, flérri par linjuste scepticisme, et condamné par ceux qui voyoient dans son action, ou l'écueil d'un système, ou le renverseurient d'un plan routinier. Il falloit un conflict d'opinions, pour que la vérité pût éclore; il falloit du temps, dẹ la réflexion, del'expérience pour donner invariablement au quinquina le titre de remède utile et précieux. Mais, le dirons-nous, des connoissances acquises sur les riches propriétés de ce médicament, sont sortis la prévention, l'abus, et par conséquent les maux d'une application vicieuse; aussi a-t-on avancé fort judicieusement (I) que l'usage du quinquina, porté pour la guérison précoce des fièvres, et pour les doses jusqu'à l'abus le plus effrayant, est un enthousiasme de la fin de ce siècle qu'il est bon au moins de faire remarquer.

LVII. Pour perfectionner l'usage qu'on devoit faire du quincina (2), il étoit naturel qu'on

(I) M. de Horne, journ. de Méd. mil. t. 3, p. 356 .

(2) Nous parlons ici du quinquina ordinaire, c'eft-àdire, du quinquina du Pérou ou de celui de Santa fé, qui eft abfolument le mêne. Le quinquina-piton en diffère à quelques égards, comme on le verra lorfque nous parderons de cet autré fébrifinge. Yoyez da nose du d. cyu. 
122

De l'usage du Quinquina

cherchât à quels principes ce médicament doit ses propriétés éminentes. La chimie les a fait pressentir ces principes. Elle apprend qu'un peu de terre, un peu de gomme, un peu de résine, le tout joint à une substance parenchimateuse, ligneuse, forment un composé, en total, plus résineux qu'extractif, et dans lesquelles les parties gommeuses et résineuses sont intimément unies ensemble, ainsi que dans la plupart des substances végétales. En procédant ensuite séparément sur chacun de ces principes, on voit que la partie terrestre est une terre très-fine, sans saveur, a'une couleur d'un jaune très-pâle, laquelle se dissout avec promptitude dans les acides et se trouve plus abondamment contente dans la partie de l'écorce qui tient à la partie ligneuse de larbre. On voit que la partie résineuse, privée de toute la portion extractive qu'elle peut contenir, est une substance friable au toucher, d'une couleur de tabac' d'espagne, sans odeur, sans saveur, répandant sur les charbons ardens une vapeur blanche assez épaisse, légèrement aromatique, colorant à peine l'eau distillée, et se disa solvant presqu'en entier dans l'esprit de vin, à l'exception d'une très-petite quantité d'une tère subtile, rougeâtre, provenant de la décomposition de la résine, qu'on retire plus abondamxent de la partic extérieure de cette écorce 
on voit enfin que la partie gommeuse est une substance extractive de couleur d'hyacinte, d'une saveur très-amère, presque entic̀rement soluble dans l'eau, et insoluble dans l'esprit de vin et l'éther; laqulle se trouve en plus grande quantité dans la partie de l'écorce qui tient à la partie ligneuse de l'arbre.

Démontrés par les meilleurs procédés analytiques, ces principes manifestent dans le quinquina des facultés astringentes et toniques; mais nous instruisent-ils sur cette vertu fébrifuge que des observations exactes et faites avec justesse, ont établi contre l'opposition la plus marquée? Cette prérogative spécifique est-elle l'effet de l'action astringente et tonique du médicament, comme le pensent quelques-uns? Dérive-t-elle de ses parties volatiles, ainsi que d'autres le présument, fondés sur ce que le quinquina réussit mieux dans le pays de sa naissance, et que son extrait même, fait habilement sur les lieux, opère parmi nous d'une manière plus prompte et plus complette? Doit-on la placer exclusivement, ou à un plus haut degré dans la partie résineuse, suivant lopinion de certains Auteurs, ou vaut-il mieux augurer que la subsitance terreuse en est la partie essentielle, mais que le quinquina n'agit jamais plus complétement que par tous ses principes réunis, parce gualors une propriété trop active 


\section{De l'usage du Quinquina}

est justement modérée par les autres? Nous ne le déciderons pas. Mais si l'on nous forçoit de prononcer sur une matière aussi obscure et dénuée de faits positifs, nous dirions, sur l'aperçu, que les miasmes marécageux agissent en partie par une acidité ( $\$ . \mathrm{XV}$.) virulente; qu'en effet le quinquina doit sur-tout opérer par sa terre absorbante secondée par la réunion naturelle de tous les autres principes. Nous serions fondés sur ce que l'écorce qui surpasse en bonté tous les fébrifuges connus, contient aussi beaucoup plus qu'aucun d'eux, de cette terre absorbante, sans néanmoins être à la tête des fébrifuges amers, aromatiques, styptiques. De l'union de cette terre avecle levain févreux, nous verrions résulter un sel neutre soluble qui procure les excrétions critiques, sans lesquelles le quinquina ne produit pas de franches guérisons. Et comme l'action du levain sur les solides est réellement affoiblissante, d'après les effets connus du gaz acide carbonique sur la fibre animale, comme le ton des solides doit être d'autant plus abattu qu'il a été porté à un très-haut degré par l'action de la fièvre, puisque l'afraissement est toujours relatif à l'intensité de l'érétisme qui a précédé, il s'en suivroit que les principes gommo-résineux allroient encore une utilité directe, parce que, en agissant en qualité d'amer et de tonique-astringent, 
dans les fièvres rémittentes.:

ils remontent tous les mouvemens dusystème, et contrebalancent l'effet très-souvent énervant des substances salines. Ainsi notre opinion seroit fortifiée par cette expérience qui constamment a démontré que, lorsqu'on vouloit une action prompte, un effet décisif, il falloit donner, ou la poudre de quinquina, ou son extrait aqueux, comme étant les préparations les plus énergiques ( $\$$. XXXVIII.)

On opposeroit en vain, si nous souscrivions de bonne foi à la vérité d'une explication plus ingénieuse peut-être que solide, que le quinquina n'a pas une vertu directe contre la cause fébrile; puisquil ne guérit pas toutes les fièvres, qu'il en prolonge quelques-unes et en fait dégénérer d'autres. Mais l'objection ne seroit-elle pas résoute, en répliquant que le levain fiévreux n'est pas toujours accessible au quinquina en vertu des complications ou des effets de la fièvre; que ces complications et ces effets de nature à être envénimés par le quinquina, prédominent dans quelques circonstances sur les indications du levain fébrile; et que, si le quinquina opère de fâcheux accidens pour avoir été mal placé, ce vice d’administration ne peut rien faire imputer légitimement au fébrifuge?

Le retour de la fièvre dissipée par le quinquina, ne formeroit pas une opposition plus 


\section{I26 De l'usage du Quinquina.}

valable, parce qu'il est probable, que lorsque ce spécifique ne peut atteindre la cause matérielle de la fièvre, il peut cependant en arrêter pour un temps les effets, en modifiant les oscillations des solides et les mouvemens des fluides, et en changeant la disposition actuelle du corps, surtout en fortifiant le système; de sorte que le quinquina offre décidément deux propriétés réelles, l'une fébrifuge curative lorsque le remède attaque le levain, l'autre fébrifuge palliative lorsque les circonstances font éluder ce combat et que ce remède ne peut déployer que sa vertu puissamment tonique.

Et comment le quinquina guériroit-il quelquefois comme subitement, sans péril et sans rechûte, les fièvres rémittentes les plus dangereuses, s'il n'agissoit sur un levain fiévreux par une propriété décidée spécifique? Comment les accidens les plus terribles de ces fièvres n'auroient-ils lieu que pendant les paroxismes, pour disparoitre durant la rémission, lorsque les lésions consécutives n'ont point encore été trop fortes pour être durables, sils ne dépendoient d'un levain particulier qui, mis en mouvement, se dépose ou bien affecte de préférence tal ou tel organe, à raison des circonstances qui décident son impulsion? Auss peut-on poser en fait que le quinquina est le spécifique de la cause matérielle des fièvres rémito 
sentes; conséquemment, qu'il est toujours indiqué en vertu de cette cause, tant que des contrindications majeures ne s'opposent point à son emploi. De là est venu ce précepte donné par des Auteurs recommandables : Lorsque l'on remarque une rémission apparente, nous pouvons adninistrer le quinquina avec confiance et succès. Ce n'est pas, car nous devons le faire observer, pour aller au devant des ábus d'une proposition générale, qu'une fièvre rémittente ne puisse être guérie, sans le secours du quinquina. Au contraire, nous pensons qu'il est plusieurs de ces maladies qui peuvent être combattues sans l'assistance du spécifique, parce qu'on obtient la destruction du levain fébrile par les moyens qui guérissent en qualité de correctifs des humeurs, et comme détruisant le foyer nécessaire à l'explosion du miasme fébrile.

LVIII. Mais quelle que soit l'efficacité du fébrifuge, la raison et l'expérience nous enseignent que ce médicament ne remplit, ni les indications que présentent les trois temps constitutifs d'un paroxisme, ni les indications qu'offre la première stade d'une fièvre rémittente quelconque. Dans le paroxisme $\left(\mathfrak{S} . V_{.}\right)$, c'est d'abord un spasme violentà lever, une chaleur forte à combattre, quelques crises à favoriser. Dans la premièr stade de la fièvre, ce sont une pléthore à enle- 


\section{I28 De l'usage du Quinquina}

ver, des engorgemens à détruire, des fibres roides à fléchir, des solides érétisés à détendre, ou des forces vitales à relever, des humeurs fixes à délayer, ou des évacuations à produire, et une égalité d'action à établir. Le quinquina iroit donc contre le but qu'il faut se proposer. Par son action tonique, il complétercit les engorgemens et appelleroit l'inflammation; par son astringence, il supprimeroit les évaciations, symptomatiques à la vérité, mais nécessaires pour alléger la nature: car, quoique le quinquina puisse exciter quelquefois l'action des intestins à se débarrasser des matières excrémentitielles dont ils sont surchargés, il n'en est pas moins vrai que ce n'est pas là la manière ordinaire et naturelle d'agir de ce remède, et qu'il resserre communément le ventre par son astriction. D'ailleurs l'effet évacuant du quinquina n'est point à désirer, lorsqu'il résulte d'un excès d'irritation, ou d'une dégénération ultérieure des liquides.

LIX. Des médecins ingénieux, guidés d'ailleurs par la théorie et les accidens propres aux frissons, frappés des phénomènes sympathiques qui, des entrailles se répètent réciproquement sur la peau, et, plus que tout, appuyés sur ce que le spaşme cesse aussitôt qu'une sueur modérée se répand également sur toute la surface du corps; ce qui leur fait considerer la sueur comme une suite 
stitite de la cessation du spasme, et du rétablissement de l'égalité et de la liberté de la circulation dans les derniers vaisseaux capillaires; préaendent que pour lever le spasme, pour le prévenir ou pour en abréger la durée, il ne s'agit que d'agacer le ventricule, et d'établir dans les organes épigastriques, un spasme fixe révulsif de celui de la peau. A cette intention, les uns ( 1 ) recommandent de donner l'émétique au commencement ou aux approches du froid de l'exacerbation; convaincus qu'outre l'évacuation des matières nuisibles des premières voies, il se fait, au moyen de la secousse, des nausées et du vomissement que procure ce remède, comme une espècè de détente qui porte les mouvemens du dedans au dehors; et qu'en même temps la chaleur se répand à la surface, les extrémités vasculaires se relâchent, le spasme se dissipe, et la peau $s^{3}$ humecte de la matière de la sueur ou de celle de la transpiration, de la même manière que cela arrive dans la terminaison des accès de fièvres par les seuls efforts de la nature; en sorte que

(I) Cullen firft lines of the pratice of Phyfic., \&xc. t. I; Phyjical and litterary eflays tom. I i art. 7.; Franklin; letters and papers an philo fophical fubjects p. $360 ; 1$ 'auteur des reflections on the general treatmen and cure of fevers, \&c. $8 x$ avant eux Riviere, Vanhelizont, lib. de febrib, cap. ?. 


\section{T30 De l'usage du Quinquina}

l'émétique peutêtre regaraé comme évâcuant et antispasmodique tontensemble. Sans s'écarter dúfond de ce système, d'autres (I) assurent qu'il suffit d'administrer les émétiques à dose sufísante pour cunser des nausées continuelles, parce qu'il est indigérent, pour l'effer fébrifuge de ces médicamens, quils occasionnent ou non des évacuations sensibles.

II se trouve enfin une troisième classe de Médecins (2) qui, pensant avec $V$ anhelmont, que les diaphorétiques seuls sont les remèdes spécifiques ct appropriés des fièvres, ont seulement en vue, sans exciter ni nausées, ni vomissement, de diriger constamment l'ordre des mouvemens du centre à la circonférence, par l'administration réitérée de quelque préparation antimoniale, teile que la poudre de James (3) ou le tartre

(I) Voyez cncore l'auteur des reflections, \&c.

(2) Voyez fur-tout Guillaume Withe, obfervations on the use of dr. jame's pouter and uther antinonical preparations in fever, \&re., les ouvrages de MM. Clark, Lind, Home, \&x.

(3) La poudre de James, comme nous l'apprend Donald Monro (a treatife on medical and pharmacentical chymifiry, \&c.), n'eft autre chofe que de l'antimoine calciné avec une fuffifante quantité d'huile \& de fels animaux, bien Jéphlegmés, bouilli enfuite dans du nitre fondu, dent on le fépare par le lavage. Cette poudre à laquelle 
dans les fières rémittentes.

stibié seul, ou combiné avec les narcotiques (I), assurant que par cette méthode ils font disparoître, comme par enchantement, la constriction spasmodique de toute la surface du corps; ce qui; selon eux, paroît dî̀ à l'action particulière que les antimoniaux ont sur les nerfs de l'estomac qui agissent ensuite sur ceux de l'habitude.

Cette constriction spasmodique étant dissipée par ces médicamens, c'est-à-dire, dès la première

l'auteur affocioit d'abord une préparation mercurielle, qu'il profcrivit enfuite, eft d'un lifage très-familier en Angleterre contre tontes fortes de fièvres, \& plufieurs Médecins en ont célébré les vertus; d'autres Praticiens fe font néanmoins élevés contr'elle. M. Guil. White (loc. cit.) lui préfère le tartre ftibié, parce que l'on connoît fa force \& que l'on peut calculer fá dofe avec exactitude \& précifion. M. Clark (obfervations on the difeafes in long voyages to hal countries, \&ac.) obferve qu'elle eft moins sûre que le tartre ftibié, \& que les évacuations qu'elle caufe, fur-tout par les fueurs, la reudent très-préjudiciable dans les fièvres purrides des climats chauds. Le jugement de M. Sims (ob. fur les malad. épidém. p. 32,45, 146.) ne lui eft pas favorable. M. Home (clinical experimenz hiftories and difections, \&c., fect. 2) en fait un parallele avec le zartre ftibié, \& dit que celui-ci eft à préférér dans les fynochos, \& la poudre dans le typhos, \&c., \&c. Il réfulte donc de tout cela qu'avec nos préprations antino. niales nous pouvons nous paffer de la poudre de James.

(I) Vogez l'aut. cité des reflections, stc. 


\section{I32: De l'usage du Quinquina}

rémission, la plupart de ces Médecins conseillont de prévenir le retour des paroxismes fébriles. par l'usage des toniques et des remèdes qui diminuent lirritabilité, sur-tout du quinquina; de donner ce fébrifuge en aussi forte dose quion le prescrit contre les fièvres intermittentes et les fievres putrides; si le paroxisme revient, on recommence le même traitement, et on se comporte ainsi jusqu’à ce que la maladie ait cédé.

Une méthode aussi active, faite pour maitriser la nature, n'est sans doute pas celle qui convient au plus grand nombre des cas. Nous savons à la vérité qu'on n'en connoîr pas de meilleure contre certaines fièvres causées par le levain le plus actif, telle quel'épidémie dont parle Senac (I) dans laquelle la mort survenoit à la troisième ou quatrième exacerbation; contre ces fièvres, que l'on ne voit guères que dans les climats les plus chauds, marécageux, pestilentiels, et dans lesquelles la férocité du mal exige qu'on s'oppose ausecond, au troisième ou au quatrième paroxisme, quelquefois au premier, ainsi qu'on l'a vu dans la fièvre rémittente de Batavia, qui tuoit dans la première attaque, et qu'on ne pouvoit prévenir qu'en administrant le quinquina comme

(i) De reconditâ febrium \&x. naturá, pag: 410. 
dans les fièvres rémittentes.

prophylactique, en aussi grande quantité que. l'estomac pouvoit le supporter (I).

L'observation suivante offre un cas des maladies de cette sorte.

$\left(^{*}\right)$ M. Nogarede, Bourgeois, après avoir été exposé quelque temps à de vives alarmes, et humé l'air d'un atmosphère marécageuse, est saisi du frisson fébrile. Le premier paroxisme est assez fort, cependant il est régulier, et dès qu'il est fini, je donnel"émétique qui opère par le haut et le bas avec succès; le second redoublement est accablant, il débute par une syncope, le froid dure quatre heures, la tête s'embarrasse, dans le chaud le malade est sans forces, il délire, sa langue est humide, très-chargée, le ventre est souple, la chaleur du corps très-modérée. On applique des vésicatoires aux jambes et l'on place, pendant la rémittence, un purgatif qui amena des selles abondantes, bilieuses, épaisses et très-férides, les urines couloient bien. Le troisième redoublement qui survint le troisième jour de la maladic est beaucoup plus grave que ceilui de la veille; le froid mêlé de frisson est long avec de légères défaillances. Lo malade est dans le plus grand accablement, le pouls est misérable; dans le second

(I) Voy. Loind. Mém. fur les fièvres \&x fur la contagion, pag. 260 


\section{De l'ufage du Quinquina}

temps du paroxisme, il y a du délire sourd. Les plaies des vésicatoires sont gangrénées. Ce redou- blement étant fini, je donne deux onces et demi de quinquina: savoir, une once en poudre partagée en quatre doses, délayée dans une décoction faite avecune once et demi de quinquina. Contre l'attente commune, le quatrième redoublementest prévenu. Lecinquième jour eśt destiné au repos; la fièvre continue persiste, les signes de saburre sont les mêmes, mais la tête est libre et tous les symptômes sont très-modérés. Je répète le purgatif le sixièm jour, et le lendemain le malade prend une once de quinquina en poudre. $D_{\text {il }}$ buitième jour à la fin de la fièvre qui a duré 2 I jours, il a fallu purger par intervalles, et le malade est parfaitement guérit.

Cet exemple est sans doute très-démonstratiff; mais ce quii le rend tel à nos yeux, c'est que $M$. V... malade dans le même temps, et dans des crrconstances analogues, meurt victime de la même maladie. Ses Médecins insistèrent trop sur les évacuans, et lorsquilis eurent recours au quinquina, ils le domèrent seulement à la dose de quatre drachmes, et trop tard. Le redoublement qui suivit, et pendant lequel je fus appelé en consultation, se termina par la mori.

Cependaat de pareilles maladies sont peu communes dans nos climats tempérés, ou dia mojins 
dans les fièures rémitientes.

elles y sont moins violentes; aussi un traitement aussi actif leur convient moins généralement. Si limitation peut et doit être permise, c'est dans le cas où la maladie ayant été négligée, le Médecin présume par les signes passés et les indices concomittans que l'exacerbation qui commence. peut être mortelle. En s'élevant alors au-dessus du préjugé, il donnera l'émétique, comme le senil antispasmodique approprié, et placera do suite le quinquina comme l'unique moyen de sauver son malade.

Une pratique moins sujette à restriction $\in \hat{t}$ plus généralement convenable pour diminuer et abréger le spasme fébrile, c'est de donner de temps en temps de petites quantités de boissons chaudes, diapnotiques, telle qu'une infusion de fleurs de pavot rouge et de camomille, et de faire en même temps des fomentations humides vaporeuses (I) sur les extrémités inférienres.

(I) Ces fomentations, précérables aux pediluves $8 x$ aux topiques, confiftant en des vefies à demie pleines d'un liquide chand émollient, s'exécurent avec une aifance fingulière en fe fervant des briques ou des tuiles qu'on fait échanfer le plus qu'on peut, qu'on trempe enfuite dans de l'eau, d'où on les retire prefqu'aufiitôt pendant qu'eiles font chaudes, pour les envelopper dans une flanelle \& les appliquer a la plante des pieds \& le long des jambes. Les briques confervent la chaleur pendane 


\section{T36 \\ De l'usage du Quinquina}

Quand l'intensité et la durée du frisson exigent des secourrs plus actifs, on se sert très-fructueusement d'un mélange d'eau de chardon bénit ex de vin émétique, quon donne par cuillerées assez rapprochées pour exciter d'obscures nausées. On emploie de même dans le pretiier temps du pam roxisme, le camphre, le castoreum, la liqueur d'Hoffmann, l'alkali volatil, l'éther ou le laudanum liquide dans les eaux cépháliques et cordiales. Quelquefois il arriveque, pour s'opposer aux accidens qui se déclarent dans l'espace intermédiaire du premier et du second temps, il faut recourir au bon vin animé par quelques gouttes de lilium de paracelse.

LX. La chaleur, qui forme le second temps du paroxisme, est, pour l'ordinaire, d'autant plus forte, que le froid qui constitue le premier temps, a eu plus d'intensité. La sécheresse de la bouche et de la peau, la petite quantité des urines rouges et ardentes, entr'autres signes, manifestent assez la continuité du spasme, quoique les autres phénomènes en démontrent la forte diminution. C'est dans cette persévérance et ce degré du spasme, que des praticiens trou-

long-temps. On peut les changer fans gênerle malade, \& l'immerfion préalable dans l'eau, leur donne une cerfaine humidité, dont l'exhalaifon eft très-émoliente: (Lind): 
dans les fièyres rémititentes. vent une raison pour placer l'émétique dans le temps même de la chaleur, sous prétexte de produire, avec ce remède, plus immédiatement ou plus prochainement, la sueur qui amène la rémission ou le temps propre à l'usage du fébrifuge. Nous nous répéterions, si nous voulions discuter la validité de cette indication ( $\$$ LIX). Le temps de la chaleur n'est propre, ni à l'administration des vomitifs, ni à celui des fébrifuges. Le danger de mort, où sont les malades dans les cas les plus périlleux, autorise seulement l'usage de ces divers moyens: ainsi' Sandifort se vit contraint, dans une fièvre qu'il eut à traiter aux Barbades, de domner incessamment le quinquina dans un paroxisme fébrile, et Gleghorn l'avoit pratiqué avant lui à Minorque (I).

Les indications directes de la chaleur sont autrement de modérer l'efervescence des liquides par la saignée ( $\$$ LXII.), par les boissons prises fraiches, par les lavemens, par les pédiluves, par un atmosphère bien âcrée. Les acides ont sur-tout une propriété décidée contre la chaleur fébrile; on les mêle aux tisannes, anx juleps; on les incorpore dans la matière des là-

(1) Obfervations on the epidemical difeafes in minorica. 8xc., Lind, loc. cir. p. 242 , 


\section{I38. De l'usage du Quinqnina}

vemens, dans l'eau des pédiluves, et dans celle qu'on peut mettre en évaporation autour du lit du malade. Le nitre jouit pareillement ici d'une grande réputation, soit seul, soit uni à de petites doses de camphre. En un mot, il n'est pas de rafraichissans qu'on ne puisse employer avec succès dans le second temps du paroxisme.

LXI. Dans le troisième, destiné aux évacuations critiques qui terminent l'orage, que peuton faire de mieux que de rester dans une salutaire expectation? Une boisson un peu chaude facilite les excrétions qui se font par la peau; cette boisson détrempe, et elle seconde le cours des selles; elle délaye, et le cours des urines en est favorisé. Disons mieux, c'est la nature qui fe décharge par quelqu'émonctoire; il ne s'agit que de ne la point contrarier.

Mais les indications des moyens qui doivent précéder l'usage du quinquina s'étendent bien au-delà du paroxisme. Elles subsistent durant toute la première stade de la maladie; et la longueur de cette stade est relative ou subordonnée à plusieurs circonstances. Ce temps est réservé pour l'administration des remèdes appelés généraux: remèdes qu'il faut encore placer avec discernement et une sage économie.

LXII. La pléthore est une des indications qu'il 
importe le plus de remplir. La masse des liquides, augmentée et rompant l'équilibre, est un poids si accablant pour la nature, qu'on trouve quelquefois les principaux symptômes de la résolution des forces, chez les sujets dont ces forces ne sont cependant qu'opprimées. La pcail des malades est fraiche, quelquefois suante; on eft menacé de syncopes et même il s'en déclare quelquefois de légères; le pouls est petit, lent, mais l'artère est dure : il falut saigner pour que la fièvre se développe, il faut alléger l'action systaltique des vaisseaux pour relever les forces et développer la maladie. On réitère la saignée et l'on proportionne l'effusion du sang, ì la force, à la dureté du pouls, au tempérament du malade, aux circonstances antérieures (v. $§ L X V I)$ à la fièvre, enfin à la qualité du sang qui a été versé dans les pálettes.

Le plus hat point du paroxisme est sans doute l'époque où la réunion des accidens cxige la saignese. Mais qu'on ne s'y trompe pas. Cette époque est aussi celle où quelquefois le sang, simplement raréfé, ne foumit qu'une indication fausse et indirecte de saigner le malade. Ici, le pouls, quoique grand, n'est pas dur, l'artère, quoique se dilatant avec force, est molle et le tempérament n'annonce rien de pléthorique. C'est pendant la rémission que les indices de plé 


\section{De l'usage du Quinquina}

thore ne sont point équivoques, et tout médecin qui ne tirera pas ses indications, pour saigner une ou plusieurs fois dans le paroxismè, de l'état du pouls pendant la rémission, sera souvent exposé, s'il n'a beaucoup d'expérience, à prendre certains accidens d'une grande raréfaction dusang, pour ceux d'une pléthore mue; mais, comme on le pense bien, ce sera au détriment du malade. Les saignées déplacées font un double mal, celui de retarder la coction par la foiblesse du malade, et celui d'augmenter l'intensité des reprises par un effet de cette même foiblesse; car on s'est souvent convaincu que le paroxisme est d'autant plus violent, que le malade a donné pendant la rémission des signes d'une grande foiblesse.

En tirant à propos le sang, soit pendant le paroxisme, soit durant la rémission, on remplit, par un moyen qu'il est impossible de remplacer, une infinité de vues essentielles. On épargne à la nature le soin pépible de déterminer des hémorragies critico-symptomatiques, on prévient les engorgemens et l'on détruit ceux qui sont formés; les solides érétisés par les premières impressions de la fièvre, sont distendus; un relâchement salutaire succède à une fâcheuse tension, et l'action des vaisseaux ayant acquis une juste liberté, les produits excrémentiviels des 
la fièvre sont infiniment moindres. Ajoutons que les humeurs étant travaillées par une force proportionnce, la coction commence de bonne heure, et le cours du mal est heurausement circonscrit.

LXIII. Secondée par les adoucissans et les tempérans, la saignée n'est, comme on le pense bien, indiquée que dans les cas, assez communs à la vérité, d'excitation des forces. On administre des moyens bien opposés lorsqu'il s'agit de relever les polivoirs vitaux, qui est lindication majeure pendant le cours d'une fièvre rémittente maligne, et pour l'ordinaire dans certains temps de toute fièvre rémittente simple. Les analeptiques, les cordiaux et les irritans remplissent cette indication, et de préférence on les emploie avant le paroxisme, à la fin du froid et pendant la rémission. Avant le paroxisme il faut penser à modérer ce froid glaçant dont les effets sont si formidables. La fin du froid est quelquefois le moment où la vie s'éteindroit, si des secours les plus actifs ne venoient point $\mathrm{z}$ l'aide de la nature; et pendant la rémission, si les forces, qui languissent en raison directe de la violence du dernier redoublement, ne sont point excitées, le danger devient et plus grand et plus imminent de reprise en reprise.

De simples potions composées avec les infu- 


\section{\$42 De l'usage du Quinquina}

sions ou décoctions de scabieuse, de camomille; de chardon bénit, d'angélique, d'impératoire, ou de contrayerva; renforcées par les confections d'hyacinte ou d'alkermes, la thériaque, le musc, les alkalis volatils et tous les spiritueux; adoucies avec les sirops d'cillets, de scordium, d'écorce d'orange, 8xc., forment les moyens connus qui réussissent dans les cas urgens où la nature défaillante et prête à succomber, demande à être ranimée par des excitans héroiques. Mais ces secours subsidiaires ont une action trop bornée, trop passagère, pour remplir le but que présente la débilité de la rémission. Le quinquina comme tonique, la serpentaire de Virginie, les vésicatoires sont trop souvent contrindiqués, ou leurs effets sont insuffisans et peu durables. C'est donc ici le cas de placer les antimoniaux, tels que la poudre de James, ou le tartre émétqque, puisque sur les aperçus de M. Home (i), ces médicamens agissent, selon toute apparence, comme des stimulans qui portent leur action dans toute la machine: aussi le temps le plus propice à leur succès, est lorsque le redoublement de la fièvre est fini, et dans ce cas où le pouls est plus foible que l'état général des forces ne semble l'indiquer, on donne le

(I) Loco citato. 
dans les fièvres rémittentes.

remède adopté, à doses très-refractées, et on 18 combine avec de petites quantités de laudanum sil agissoit par le vomissement ou par les selles, car toute évacuation est alors énervante lorsqu'elle n'est point demandée par la nature de la maladie.

Les fomentations des jambes et les pédiluves peuvent encore être employés dans la vue d'exciter la machine, pourvu que la chaleur de l'eau soit plus considérable que celle du corps. $M$. Home prétend que ces moyens ont un effet stimulant et relèvent le pouls d'une manière trèssensible. Le docteur Armstrong en vante les avantages, sur-tout lorsqu'ils sont suivis des lotions avec du vin chaud ou de l'eau de vie (I); \& M. Parc, qui ne leur refuse pas cette propriété, nous prévient qu'ils agissent d'autant plus comme des stimulans, qu'après avoir fait chauffer l'eau du bain jusqu'au 106e. degré du thermomètre de Fharenheit, on en augmente ensuite la chaleur, s'il est possible, jusqu'à ce que le malade se plaigne de nausée et de vertige (2).

LXIV. De quelque nature que soit la maladie; il n'est que du plus au moins indispensable de remplir, par l'usage des boissons, l'indication

(1) Médioal efjay, \&c.

(2) De Balneo difr. Edinbourg I 773 . 
de délayer les fluides. Dans les premiers jouts de l'attaque fébrile, qui est le temps de la plus grande crudité, le spasme et la phlogose forment le pius souvent les efiets du mal, les excrétoires sont resserrés, les fibres sont crispéés ou souffrent de la tension que les fluides raréfiés procurent. Si les malades refusent de boire les progrès de la fièvre sont rapides, la tête se prend, le délire survient, la langue se sèche et noircit, le pouls acquiert de la dureté, en un mot, l'érétisme et l'inflammation sont portés au comble. C'est ce qu'on observe quelquefois dans les hôpitaux (I) où l'impossibilité des soins proportionnés fait que certains malades passent les dix à douze heures sans boire.

Mais il ne faudroit pas s'y méprendre. Trop de boisson expose de même aux accidens les plus fâcheux. Ce torrent de liguides pèse sur des parties trop érétisées et qui ne peuvent aussi subitement lui livrer passage; il fatigue donc, par son poids, l'estomác qu'il énerve par sa propriété. Sil entre daris les vaisseaux, c'est une abondance de liqueurs crues, incapables de s'assimiler avec le sang, mais propres à relâcher ou tendre les parois des vaisseaux, à engorger

(1) Voy. M. Arnaut de Nobleville, cours de Médo prat. tom. 1I, P. 379. les 
dans les fieyres rémittentes.

145.

les viscères, sur-tout les poumons, et consécutivement à transmettre, au système nerveux á un degré funeste de constriction ou d'atonie. Aussi des praticiens éclairés ont-ils imputé d l'excès des boissons, d'occasionner du mal-aise de rendre le corps lourd, de fufciter des nausées le délire, l'affection soporeuse, des tremblemens dans les membres, des foiblesses avec menaces de syncope, le hoquet, des vents, le météorisme; l'érétisme du genre nerveux et membraneux, le retard de la coction, en un mot les mêmes effets que produisent communément les échauf fans et les âcres (1).

La plus forte ardeur du paroxisme n'est pas même, dans certains cas, trne raison de prodiguer. les boissons délayantes, parce qu'il est à craindre: que cette abondante quantité d'eau, venant à séjourner dans l'estomac pendant l'exacerbation ne serve qu'à détremper l'humeur septique qu s'y trouve stagnante, et à la rendre par-là plus. active, plus pénétrante et plus propre à entrer dans les voies de la circulation, au moyen du véhicule qu'elle trouve. Telle est, suivant $\mathrm{M}_{\text {: }}$ de la Berthonie (2), là dure épreuve qu'on fit

(i) Voy. Langrish, Tifot, le journ. de Méd. t. III, p. I $34, \&$ t. IX, p. 229 , Lorry de morborum mutatic nibus, p. 270, \&xc. , \&c.

(2) Journ. de Méd. t. XVI, pag. $2 \sigma_{2}$. 


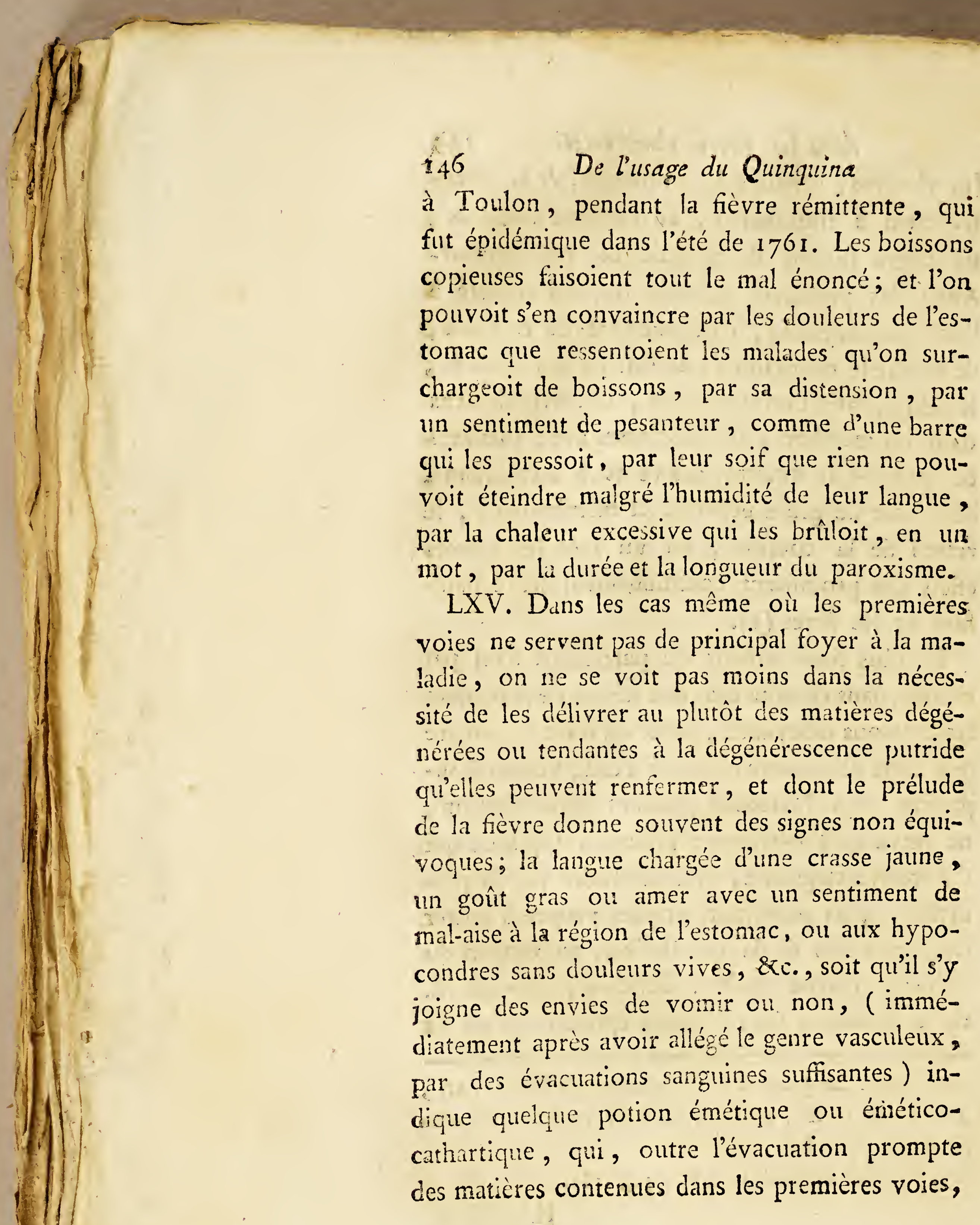


excite encore le dégorgement de celles qui croupissent dans les réservoirs et conduits excréteurs des viscères glanduleux circonvoisins, et notamment du foie.

Il est même des cas où l'on doit y avớr recours avant la saignée, savoir, lorsqu'un pouls petit, foible et déprimé, joint à de fréquentes envies de vomir, et au sentiment d'un poids incommode sur la région de l'estomac, indique que les fonctions vitales souffrent considérablement par la présence des matières dépravées: Outre les effets de l'évacuation, il résulte un autre avantage des émétiques administrés en pareil cas; ils réveillent le ton engourdi du genre nerveux et raniment l'action systaltique languissante dans le système vasculetrx par les secousses gu'ils excitent dans l'estomac et dans les autres parties qui concourent au vomissement. Il est de fait que c'est-là très-souvent le principal fruit que l'on retire des émétiques dans les fièvres malignes, et l'on doit en être persuadé, lorsqu'on voit les malades sensiblement mieux après leur action, quoique les matières évacuées soient réduites à peu de chose.

Les circonstances doivent décider de la préférence qu'il faut donner au tartre émétique, sur l'ipécacuanha et réciproquement. L'auteur des réflexions sur le traitement des fièvres en

K 2 


\section{$\$ 48$ De l'usage du Qninquina}

général, conseille l'usage de l'ipécacuanha, lorsqu'il s'agit de faire vomir, et du tartre stibié, s'il n'est question que d'exciter des nausées. L'ipécacuanha est en effet un remède précieux, s'il est vrai, ainsi que nous l'apprend $M$. de Monchy ( $\mathrm{r}$ ), qu'il soit un excellent secours toutes les fois que le principe vital, affecté directement, perd sa vigueur et son activité. C'est alors qu'on trouve, dans ce médicament, la vertu fébrifuge que lui ont reconnu Gianella (2) et M. Darluc (3) dans le traitement d'une fièvre rémittente très-grave qui fut épidémique en I76I, dans plusieurs cantons de la Provence. Quand on se voit obligé de tempérer l'action du vomitif ou de choisir une émético-cathartique, on ne sauroit mieux faire que de le composer de deux onces de manne et de dix à douze grains d'ipécacuanha; et lorsque le dépot épais, tenace et abondant qu'on trouve sur la langue, fait penser que ce vernis se propage jusques dans l'estomac, et qu'il peut protéger cet organe contre l'acti-

(1) De medico optimo nature \& obfervatore \& imiatore. Cap. V.

(2) Carali Gianella de admirabili ipecacuanhe virtute in curandis febribus, tum autumnalibus, tum lentis, tum aliis fivè continuis, fivè intermitientibus, fedem in primis viis habentibus; apud Haller, difp. morb. T. V. p. 2 I.

(3) Journ. de Méd. t. XVI, p. 36z. 
vité nécessaire du vomitif, il est bon, suivant le conseil de M. Taranget, de faire précéder immédiatement le vomitif par quelques gorgées d'eau de vie.

C'est pendant le calme de la rémission, qu'il faut placer des médicamens dont l'action est tumultueuse, mais très-salutaire, lorsqu'ils ont commencé de faire vomir des matières jaunes et bilieuses, et cru'ils finissent par exciter deux ou trois selles. Les premières voies devenant libres, les forces virales se relèvent : aussi arrivet-i1 pour l'ordinaire que l'exacerbation qui suit, paroit plus forte; mais cette intensité dénote que la circulation est plus libre, et que les vaisseaux agissent plus énergiquement sur la cause matérielle de la fière.

Comme le dégorgement des parties imbibées de sucs putrides ou altérés, se fait successivement ou peit-ì-peu, on se voit quelquefois obligé, après plus ou moins d'intervalle, de répéter le vomitif pour en obtenir de nouveaux succès. Cette pratique est sur-tout indispensable dans les fièvres des lieux marécageux; et c'est faute d'en avoir senti l'importance, que nous croyons qu'on a obtenu quelques mauvais effets du quinquina, et qu'on a vu survenir des maux dont nous avons été les témoins. On seconde l'action des vomitifs, lorsque lindication est $\mathrm{K}_{3}$. 
I50 De l'usage du Quinquina

décidée, en sollicitant avec de doux purgatifs, lévacuation des matières impures et dégénérées. Faisons-en ici l'observation en faveur de ceux qui, faute d'employer des secours proportionnés, font souvent, sans qu'ils s'en doutent, une médecine dangereusement expectante; les purgatifs ne remplacent jamais les émétiques, et ne peuvent point conséquemment leur être substitués. Ajoutons que le début des maladies n'est pas souvent un prétexte d'y recourir de bonne heure. Le temps de crudité offre quelquefois tant d'erétisme ou de phlogose, qu'on risque, avec le moindre évacuant, de dénaturer le mal ou de l'empirer (I); et le temps de létat de la fièvre, dans d'autres circonstances, offre tant de tension ou de sensibilité, que l'emploi des purgatifs, trouble les mouvemens de la nature, et entraine des accidens auxquels il n'est pas facile de remédier. Lancisi et Bianchi (2) sur-tout nous en fournissent des exemples.

LXVI. Si nous examinons actuellement les. modifications du traitement général que les fiè, vres rémittentes exigent à raison des causes qui

(1) Vay. la diff. de M. Reytemeyer, de cautelis circa semediorum procipue evacuantium ufum in morbis fientibus, vel fub initio morborum, 1779.

(2) Hiftoria hepatica conflit, anni $17 \mathrm{I} 8$, p. $748 \mathrm{du}$ rom? 
dans les fièvres rémittentes.

ont préparé et accompagnent ces maladies, nous verrons que l'état des saisons et les intempéries de l'air, en donnant un caractere aux épidémies ou aux maladies sporadiques, règlent de mêne les circonstances de ce traitement. En effet, toutes les fois que le changement de la saison aura été marqué par un passage subit du chaud au froid, de la très-grande sécheresse au temps pluvieux, et réciproquement, non seulement la cause de la maladie sera très-grave, mais le traitement deviendra délicat. Les fièvres printanières, par exemple, différeront suivant la constitution de l'hiver. Si cette saison a été variable, humide, chaude, que la disette ait régné, et que le peuple ait supporté des travaux rudes et soutenus, pour lors les fièvres seront fâcheuses, tendant à la putridité, et demanderont par conséquent qu'on soit très-réservé sur la saignée, sur l'usage des purgatifs actifs et de lémétique, et qu'on prescrive une diète, un régime qui puisse convenir également à l'étar d'épuisement des malades et à ceilli de leurs humeurs. Au contraire, si l'hiver a été sec, rigoureux, si le peuple l'a passé dans une certaine aisance, ces fièvres devenues épidémiques, ont un caractère plus inflammatoire, et ceux qui en sont attaqués sont moins épuisés; alors on ménage moins le sang; les émétiques et les

$\mathrm{K}_{4}$ 


\section{I52 De l'usage du Quinquina}

purgatifs peuvent être plus souvent employés et à plus large dose, la diète austère est plus supportable, le régime antiphlogistique indiqué. Ainsi des fières automnales: si les chaleurs ont été considérables, si l'humidité de l'air, occasionné par la pluie ou les vapeurs des marais, des étangs, des rivières ou lacs, a continué avec la chaleur; si enfin le passage de l'été à I'automne a été marqué par un changement prompt et trop sensible de la température de l'air ou par des intempéries, la fièvre automnale sera d'une nature putride, plus compliquée et plus généralement répandue. Alors le régime antiputride sera spécialement indiqué, l'usage des toniques très-efficace, les saignées deviendront peu utiles et même nuisibles, et il faudra avoir attention de faire observer aux malades, me diète qui corrige la nature putrescibledes humeurs, etc. Dans tous les cas, la nature de l'épidémie indique sur quels moyens il est permis d'insister de prérérence. On a, par exemple, quelquefois observé que le quinquina nuisoit dans quelques épidémies où d'autres fébrifuges beaucoup plus légers réussissoient à merveille (I).

LXVIII, Tels sont les moyens ( $\$$. LVIII. à

(I) Voy. M. Colombier, Médecin militaire, T. I, p. $74^{\circ}$ 
dans les fièvres rémittentes:

LXVI.) généraux qu'on peut opposer aux contrindications ( $\$$. LVIII.) du quinquina. Ce but n'est pas plutôt rempli, que l'emploi du fébrifuge est nécessaire, puisque la fièvre a été réduite à son élément simple, et que sa cause matérielle est de nature à céder à l'action du médicament. Mais; d'expérience incontesiable, ce remède, tout précieux qu'il est, n'opère pas avec un égal succès dans les trois ordres des fièvres rémittentes que nous avons distingués ( $\$$. X. ). Celles du premier ordre caractérisées par le frisson au début des paroxismes, comme étant les plus légitimes, sont aussi les fièvres contre lesquelles le quinquina exerce le plus sa vertu spécifique. Les fièvres du second ordre, dont le caractère n'est jamais mieux prononcé, quelle que soit la lucidité da premier temps du paroxisme, que lorsque l'invasion de la reprise est subite, précédée de pandiculations et de douleurs de tête, et lorsque les malades paroissent alternativement dans un espace de temps limité, tantôt dans un péril imminent et tantôt lors de tout danger actuel, ces fièvres, disons nous; quoique soumises à l'indication du quinquina, résistent cependant plus ou moins de temps à son action puissante; et les fièvres du troisième ordre ne peuvent admettre ce fébrifuge, quaprès que les ressources de l'art ont ramené la fièvre au caractère légitime des fièvres rémittentes, c'estuds 


\section{I54 De l'usage du Quinquina:}

dire, qu'après que le premier temps des exacer: bations est distinct, sensible, ou, suivant les expressions de Torti, qu'après que les indices de reprises est plutôt sur la ligne du froid que sur celle de la chaleur.

On voit déjà les raisons de la différence des succès du quinquina administré dans le traitement des fièvres rémittentes prises en général. Les fièvres du premier ordre se rapprochent intimément de la nature des fièvres intermittentes, comme celles du dernier semblent se confondre dans la nature des fièvres continues. Aussi tous les bons praticiens admetten-tils comme une règle sûre, que l'efficacité du quinquina est en raison directe des rapports que la fièvre se trouve avoir avecles intermittentes et les continues. C'est ce qui a fait dire à M. Voullonne, lorsque cet habile Médecin a tenté de réduire à une loi unique tous les rapports d'utilité que peut avoir le quinquina, avec l'objet direct de sa vertu fébrifuge, qui est la fièvre en général, que, de même que la différence qu'on observe dans la marche d'une fière quelconque étudiée durant l'espace de 48 heures, en comparant l'état de la plus grande force avec l'état de la diminution la plus sensible, donne la loi dont il est question, c'est-à-dire, qu'elle forme le signe le plus universel et le moins équivoque du quinquina comme spécifiquement fébrifuge; ainsi dans 
dans les fièvres rẻmittentes.

les fièvres rémittentes, cette différence peut varier depuis le néant jusqu'à l'infini, et que l'utilité du fébrifuge croît et décroit avec elle dans une pro* portion rigoureuse.

LXVIII. Si lindication du quinquina est tracée par le caractère de la fièvre rémittente, la nécessité d'y recourir plus ou moins promptement, et de le donner à dose plus ou moins considérable, est de même déterminée par la nature des accidens ( $\$$. II.) qui se développent pendant le paroxisme, et par celle de la maladie qui peut être simple, grave ou maligne. Une fièvre caractérisée simple par les symptômes ordinaires d'une exacerbation, n'exige que peu ou point du tout de fébrifuge; les forces de la nature suffisent pour amener une prompte guérison, et l'on est presque toujours maitre de la completter, lorsque les limites du mal semblent ne vouloir pas se circonscrire. Mais dans une fièvre caractérisée grave par un ou plusieurs épiphénomènes par lesquels le danger a coutume de s'exprimer, le quinquina doit être donné de bonne heure et à grande dose. L'indication est d'arrêter la fièvre, mâme contre les considérations les plus pressantes, pour que ses efiets ne viennent pas à dominer, le moindre délai pouvant être préjudiciable et permettre à la fièvre de rentrer dans la classe de ces maladies dégénérées contre lesquelles le 


\section{De l'usage du Quinquina}

quinquina n'est plus indiqué. Quant aux fièvres décidément malignes en vertu de la résolution des forces qui les constitue telles, tout indique qu'il faut avoir recours au quinquina lors même que les redoublemens, dont le caractère rémittent est très-marqué, ne sont pas périodiques.

L'observation l'a trop souvent démontré: toutes les fois que la fièvre est une afiection grave ainsi que les autres élémens qui la constituent maligne, toutes les fois qu'elle présente la première indication, on ne sauroit l'arrêter trop tôt, ou du moins l'attaquer assez directement par le spécifique. Si l'on attendoit des signes de coction, si l'on se flattoit d'une crise, la fièvre auroit fair une victime avant le temps propre à ces événemens; et pour prix de sa confiance dans les res-

- cources de la nature, il ne resteroit au Médecin que le regret d'avoir méconnu les indications urgentes d'un médicament dont on n'avoit que des succès, et des succès prompts à attendre. Le plus grand nombre des fièvres que règnent dans les pays chauds et mal sains, sont dans la classe des fièvres dangereuses, et dont il faut prévenir les rapides et mortels accroissements; aussi voyonsnous que ceux qui les ont traitées avec le plus de fruit, ont employé le quinquina dès la première, la seconde, et, au plus tard, dès la troisième ré, mittence. 
LXIX. Que peut-on attendre, en effet, de ces fièvres corruptives et graves, dont chaque exacerbation tend à opérer une nouvelle fonte d'humeurs, à procurer l'inflammation de quelque viscère, à exciter quelques épiphénomènes également formidables et destructifs? II n'est qu'un temps pour arrêter ou détourner ces funestes produits. Celıi où il sont subordonnés à la fièvre qui les suscite; le laisse-t-on passer, le quinquina qui pouvoit tout, n'a plus le moindre effet; il devient au contraire très-dangereux, parce qu’il irrite les produits du mal, et coopère pour les faire dominer de plus en plus sur les indications de la fièvre. Nous reviendrons quelquefois sur ce précepte essentiel, parce qu'il règle invariablement, et le temps où le quinquina peut réussir, et les circonstances où l'on doit recourir de bonne heure à ce fébrifuge.

LXX. Il semble, au premier coup d'ceil, que des symptômes d'inflammation ne peuvent point être guéris et prévenus par un médicament si propre d'âilleurs à les faire naître. Mais si lon fait attention que cet appareil inflammatoire n'est que le produit de l'action fébrile, on sentira qu'en s'opposant à propos à la fièvre elle-même, on étouffera dans son germe tout ce qui peut en dériver. Sarcone a donné à ce sujet le précepte le plus lumineux et le plus concis. Lorsque lino 


\section{$15^{8}$ De l'usage du Quinquina}

flammation, dit ce sage praticien, est une suite; un produit, non du seul période que lui donne son nom, mais de la cause même qui sévit avec un ordre constant, et qui, par cette raison, est appelée périodique, pour lors l'inflammation en étant réputée comme l'effet, peut être emportée par le fébrifuge. Sur quoi l'on doit remarquer : $1^{\circ}$. que, pour qu'on puisse raisonnablement compter que le quinquina produise de bons effets et morde sur une maladie inflammatoire symptômatique, il ne suffit pas qu'il y ait du périodique; mais il faut au moins que les rémissions soient sensibles et d'une durée convenable. 2\%. Qu'il est expressément requis que l'inflammation n'ait pas jeté de profondes racines, et n'ait pas acquis ce degré d'intensité qui peut la faire remarquer comme une maladie en soi, mais qu'il se fasse journellement en elle comme une espèce de résolution et 'de renouvellement alternatifs de retour et de rémissions convenables de la fièvre (I).

C'est d'après ces considérations, que Sarcone traita en 1764 à Naples, la cruelle épidémie des fières rémittentes, dont les paroxismes tendoient constamment à procurer l'inflammation de quelques viscères; inflammation qui ne pouroit être directement attaquée que par le quin.

(1) Iftoria ragionata de mali ofervati in Napoli, \&ac, part. I, pag. I98, 192. 

quina, lorsqu'on ne lui avoit pas donné le temps de devenir essentielle et continue. Cleghorn nous apprend que, dans les fièvres tierces qui règnent très-souvent à l'île de minorque, il est impossible d'éviter une inflammation d'entrailles, et la mort qui en est une rapide conséquence, si, immédiatement après une saiguée, on ne donne le fébrifuge à haute dose. Nous citerons encore Médicus, qui a vu à Manhein des fièvres rémittentes dans lesquelles il y avoit tant d'inconvéniens, à raison d'une inflammation locale successive, à négliger le quinquina, qu'il en a été induit d donner ce précepte trop vague, savoir, que, dans tous lesicas de fièvre inflammatoire trèsgrave, le quinquina est approprié, pour v qu'au préalable on ait employé la saignée et les antiphlogistiques convenables.

LXXI. Ces préceptes peuvent être appliqués qux fièvres rémittentes, dont les dangereux résultats sont d'étendre les progrès de la putridité et de la fonte des fluides. Grant parle d'une fièvre rémittente maligne qui régna à Bergopzoom et qu'on étoit forcé d'arrêter, pour que le malade ne périt pas d'hydropisie; le sang se dissolvoit d chaque accès (I), Quarin fait mention d'une épidémie putride maligne, dans laquelle la fièvre

(I) Recherch. fur les fièrres, t. I, p. si. 
160

De l'usage du Quinquina

dégénéroit rapidement, et le malade périssois sî. l'on différoit de donner le quinquina après avoir évacué les premières voies, quoique la langue fût encore très-sale et les urines hautes en couleur (1). L'épidémie de Pésaro décrite dans Lancisi (2) offre encore un exemple de ce dernier genre; et Sims (3), qui en vit régner une pareille dans le pays de Tyrone, fait observer que le quinquina adminisiré dans de pareilles circonstances, manque rarement d'ouvrir le ventre et même avec une certaine profusion, double effet aussi salutaire que desiré, et qui doit enhardir pour l'emploi d'un médicament aussi fébriftige que tonique.

LXXII. La dominance de la fièvre sur ses prom 'duits, et des produits trop fâcheux, trop promptement funestes, servent donc de règle invariable pour bien placer le quinquina, soit que ces produits consistent en une affection inflammatoire, soit qu'ils consistent en une affection putride. Nous en donnerons un exemple, en plaçant ici les procédés curatifs de la fièvre rémittente pleuropéripneumonique dont on a vu ailleurs ( $\$$. XiII. ) la marche et les divers symptômes.

(1) Meth. medend. febrium, p. 38.

(2) De noxiis paludum efluviis, , p. 299.

[ (3) Obf. iur les mal. épidém. p. iso.

Quoiquie 
Quoique le poumon fùt l'organe qui souffroir le plus des effets de la maladie, la saignée devoit. être bornée, et ne fournir qu'un moyen subsidiaire pour passer, sans inconvénient, les premiers jours de la maladie. Ceux qui ne virent, dans les accidents péripneumoniques, qu'un prétexte de prodiguer le sang, furent très-malheureux dans le traitement de l'épidémie. Ces accidens augmentoient en proportion du nombre des saignées : cette augmentation devenoit un nouveau motif de réitérer ce genre de secours, et la pleuropéripneumonie devenant essentielle et continue, le sort des malades étoit décidé; il n'étoit presque plus possible de les arracher à la gangrène du poumon, ou à un épanchement qui les faisoit périr. suffoqués. Les malades qui coururent le moins. de danger, furent ceux qu'on ne saigna qu'une, deux, et au plus trois fois dans les quatre premiers. joürs de la fièvre. Communément ils étoient saignés pendant l'ardeur du premier paroxisme; on rẻp étoit la saignéc dans la rémission par rapport. à la gêne de la respiration, pour placer une heure après l'émétiquie en lavage, ou un émético-cathartique composé avec deux onces de manne et quinze à vingt grains d'ipécacuanha; l'état de la poitrine faisoit préférer l'un ou l'autre de ces moyens. Les boissons pectorales, mais acidulées avecl'oximel simple, les lavemens, nous fai- 


\section{*62 De lusage du Quinquina}

soient parvenir à la troisiene exacerbacion, au plus fort de laquelle on saignoit quelquefois pour la troisieme et dernière fois, parce que la douleur latérale, s'il on restoit une, étoit avantageusement combattie, ou par un vésicatoire, ou par des embrocations faites avec le liniment volatil spirituenx (I) de Pringle, ou par tout antre épispastigue. Quelquefois l'émétique étoit donné d'emblée, et la saignée se pratiquoit pendant ou après son opération. On se guidoit, pour cetre préférence, sur la dominance des phénomènes propres à la lésion symptomatique du potimon, ou à la congestion des sucs pervertis croulpissant dans les premières voies. Ces préliminaires étant remplis, nous profitions du relâche de la seconde rémission, pour placer un minoratif qu'on remplaçoit quelquếois par le tartre stibié jeté à la dose d'un ou deux grains dans la boisson des malades, et nous revenions aे ce salutaire moyen, usqu'à ce que les premières voies fussent assez. bien nétoyées, oujusqu'à ce que des symptômes urgens forçassent à ne pas différer l'emploi du fébrifuge.

Dans l'administration du quinquina, les malades étoient divisés en trois classes. Ceirx de la

(I) Prenez d'efprit volatil de fel ammoniac, demionce; d'huile d'amandes douces, une once : mêlez. 
dans les fièvres rémitientes.

première, qui, comme on le sent bien, couroient un danger pressant, passoient incessamment à son usage après la grande reprise du troi sième jour. Les deux premières doses, de deux drachmes chacune, étoient données en substance dans un véhicule pectoral, et les autres doses étoient prises en décoction. Le fébrifuge étoit suspendu pendant les deux tiers de l'exacerbation suivante, repris sur son dédin, et continué pendant la rémission de la même manière observée le jour précédent. LLorsque l'intensité des symptô. mes étoit réfrénée et que le ventre n'avoit pas ssez de liberté, nous donnions le quinquina purgatif, et ce remède achevoit la guérison de la maladie.

Ceux de la seconde classe, moins grièvement affectés, ne prenoient le quinquina qu'après l'état de la maladie. On le leur administroit en décoction qu'on prenoit soin d'adoucir avec une tisanne émulsionnée, même avec du lait đ̆amandes, et l'on mêloit le fébrifuge aux purgatifs les jours destinés aux évacuations nécessaires.

Enfin, ceux de la troisième classe ne prirent la quinquina que sur le déclin de la fièvre, d'abord ajouté aux potions cathartiques, et ensuite combiné avec des apozèmes qu'exigeoien $\hat{\imath}$, soit la fin du mal, soit les commencemens de la convalescence

I. 2 


\subsection{De l'usage du Quinquina}

Chez tous, le quinquina, favorisé par l'action des pectoraux et quelquefois par des mixtures, dans lesquelles entroient l'huile d'amandes douces, l'extrait de têtes de coquelicot ou le sirop diacode, produisoient des effets plus ou moins prompts et heureux. Les souffrances de la poitrine diminuoient; quelques crachats cuits achevoient de débarasser le poumon; et la fin de la maladie ne différoit point du déclin d'une fièvre putride-bilieuse. Des urines fétides et chargées, des selles jaunes et de quelque consistance, constitwoient les deux crises qui jugeoient complettement la maladie.

Lorsque les malades négligés ne présentoient pas encore les symptômes d'une pleuropéripneumonie dominante, il falloit donner le quinquina, comme chez les malades de la première classe, sans avoir égard à la nécessité de saigner ou de purger, qu'on effectuoit ensuite, dès qu'on s'étoit rendu maitre des accidens urgens de la maladie. La saignée qu'on pouvoit toujours placer à sz volonté, devoit être différée jusques là, parce que les sỳmptômes qui paroissoient l'exiger, n'étant que factices, étoient plus directement combattus avec le fébrifuge. Quant aux purgatifs, le quinquina en faisoit solivent l'office, en ce qu'it mettoit des organes énervés en état de se décharger des sucs qui ne les engouoient qu'en vertu 
dans les fièvres rénititentes.

de l'atonie des uns ef de la surabondance des autres.

Mais lơrsque les symptômes pleiropéripneuta moniques étoient dominans, il falloit laisser le quinquina et remplir les indications embarrassantes qui se présentoient, soit par une petite sâignée, soit par les évacuations bien ménágées ai laide du tartre stibié, par les potions huileuses animées avec le kernes et le sirop de limon; on retiroit dé bons efefs de fomentations con tinuées sur les extrémités supérieures, et dës vapeurs de camphre et de vinaigre dirigées dans la poitrine.

LXXIII. L'administration du quinquina dans les fièvres rémittentes remarquables par tout autre épiphénomènè est soumise aux mêmes règles, aux mềmes conditions. Si les saignées et les vézsicatoires paroissent d'une grande utilité contre les accidens soporeux; si l'on cherche dे calmer le violent mál de tête et le délire frénétique par l'effusion du sang ( $(1)$, et par les pédiluves ou par

(I) S'il eft jamais néceffaire de fe tenir en garde contre le défir de combattre, par des remèdes appropriés ? les fymptômes dominans des maladies, c'ent principalement dans les fièvres rémittentes, où la fièvre formant la principale indication, doit auffi être directement combattue. La Médecinc fymptomatique, portée jufqu’à un certain point, eff d'autant plus préjudiciable, que, fans enlever 
les irritans extérieurs, et sur-tout par les vésica toires aux tempes ( $\mathrm{r}$ ); si l'on oppose au vomissement excessife, le laudanum er les topiques annodins; aumétéorisme du bas ventre, le quinquina et le camphre en lavement, etc cen ne sont dà que des auxiliaires plus on moins précienx; l'é piphénomène ne dénaturant pas lindication, puisqu'il dépend du matsvais caractère de la fièvre, c'est toujours par le quinquina quil faut tâcher de le préveniz, cest par co rébrifuge quili fau argêter la marche d'un accident, qui peut, on tuer le tnalade au plus vite, ou dénaturer le mal qui

le fymptône, elle le fait, au contranie, cuclquefois offentiel, \& rend la inaladie plus compliquée, te nalade plus fóble. La faignée snérite fur-tout de n'être placée \& répétée qu’àrès le plus mûr examen.

(1) M. Home (clini calexperiments, hifories and, diffretions, \&c., fect. 2), nous apprend que les véficatoires appliqués aux tempes, font un moyen sûr d'appaifer le mal de tête. I explique leurs effets, ${ }^{\circ}$. par le flimulus applique fur des parties trés-fenfibles, très-près de l'en. droit affete, \& qui ont une communication directe avec Jes nêrfs des youx; $2^{\circ}$. par l'évacuation qu'ils procurent drectement de la partie málade, au moyen des vaiffeaux qui partent du mềme tronc, \& communiquent entr'eux pur le moyen de l'artère orbitaire, \&c. Nous nous fom. tries quelquefois fervi de ce topique avec le plus grand Eucces? 
ne peut plus alors être attaqué par le quinquina. Les livres fournissent plusieurs exemples de ces vérités pratiques.

Il est un produit de la fievre que bien de Médecins croiroient ne devoir pas être combattu par le fébrifuge, et qui cependant est de nature à céder à son action : c'est une forre dégćnération bilieuse des humeurs, qui, faisant bientôt des progrès considérables, occasionne un ictère san's lésion particulière du foie. Mais cette lésion est prochaine, et l'on doit toujours craindre que ce viscère ne s'engorge enfin, ne se pénstre de l'humour qui a tant d’analogie avec lui, et ne finisse même par s'endurcir ou par suppurér. En donnan le quinquina avant cette lésion locale (et sars doute qu'śn peut le donner, puisque la dégénération bilieuse est l'effet du mal), on réussit àla détourner, à la prévenir, a guérir même trèspromptement la maladie. Ainsi se traite la fièvie jưue d'Amérique; dont celle que nous désignong ici est un diminutif plus ou moins sensible. Cette dégénération bilieuse se manifeste après quelques redoublemens par d'énormes évaciations de bile, ou par une légere junisse, qui ne paroit d'abord que dans le fort de l'exacerbation, et par des urines très-saffranées à la fin ou dans l'intervalle des paroxismes. Elle est commune dans les malas. 
Y88 De lusage du Quinquina

'dies dont l'ardeur des redoublemens est trèsforte.

LXXIV. C'est donc faute d'employer le fébrifuge de très-bonne heure dans les fièvres rémittentes, dont les paroxismes amènent des accidens graves et malins, qu'on perd souvent l'occasion de pouvoir le placer avec fruit. Les lésions particulières ont tant de tendance à s'établir, et une fois formées, elle s'opposent si directement aux bons effets du médicament, que, lorsqu'on veut ensuite le placer, il n'agit point, ou n'agit qu'au détriment du malade. Pour règle sûre dans tous ces cas, on peut adopter que le temps de la fièvre le plus propre à l'administration du quinquina, est celui où les accidens du paroxisme cessent de devenir indifférens. Plus la fièvre sera grave, et plutôt certe indication sera décisive. 2 immerman parle d'une fièvre tierce qui tuoit à la seconde reprise, et dans laquelle il falloit placer le quinquina dès la première rémission. La maladie étoit caractérisée par une grande oppression et une forte douleur à la tête (I). Pour l'ordinaire, cependant, la fin des malades n'est pas aussi précipitée, et lon peut dire qu'en général la quatrième ou 1a septième reprise manifeste plus ou moins clairement lindication du fébrifuge.

(I) Volder erfahrung I th. p. 204 , \& Quarin de febrib. p. 24, 
dars les fières rémittentes:

LXXV. Quand on suit avec attention la nature, on est comme frappé de l'ordre constant qu'elle met dans ses opérations. Les anciens qui les avoient observées avec la plus grande saga-citć, nous ont dit que le septième accès dans les intermittentes, mérite autant de considération et a la même valeur que le septième jour des fières continues; et que le même rapport qui se trouve dans les continues entte le quatrieme jour et le septième, se remarque pareillement entre le quatrieme accès et le septième des intermittentes. Mais on sait que les anciens n'ont point assez exactement séparé ce qui convient aux intermittentes et aux rémittentes ( $\delta$ II.), pour que leurs observations ne soient pas applicables aux unes et aux autres. Sil s'élevoit quelque doute à cet égard, nous nous appuyerions sur lautorité des modernes. Cleghorn (I), dans l'épidémie de Miuorque; Lancisi, dans les épidémies de Rome (2), d'Orviette (3), de Pesaro (4); Lautter, dans l'épidémie de Laschendorf (5); M. Dariuc, dans celle de la Provence (6);

(1) Obfervations on the épidémical difeafes in minarce, \&.c., p. I 89 , chap III.

(2) De noxiis paludum efluviis, p. Is 8.

(3) Ibid. p. I89.

(4) Ibid. p. $260,278,308$.

(5) Hiftor. bienn. morbor rural \&c. , p.

(6) Journ, de Míd. t. XVI, p. 350. 
Rosen, dans les fièvres rémittentes pernicieuses d'Upsal (I); et tant d'autres, nous apprennent que le quatrième paroxisme est indicatif du quinquina: précepte essentiel, fondamental, qu'Hippocrate (2) même a donné d'une manière non équivoque.

Par conséquent, si, dans la quatrième reprise, les symptomes saggravent et deviennent dangereux; si lexacerbation se prolonge davantage, et sur-tout si le premer temps s'obscurcit plus ou moins considerablenent, il n'y a pas a balancer, le quinquina est devenu nécessaire pour arrêter cette dégénération. Bien plus, il n'y a gueres que ce moment, nous ne dirons pas pour prévenir quelque symptôme nortel, tels que Tapoplexie et autres affections soporeuses ou paralyciques, \&c., mais pour empêcher que ces symptômes ou autres d'une nature quelconque, ne prennent des accroissemens et une intensité propre à les faire dominer sur la fièvre. Dirat-on quil y a dautres indications à remplir ?

(i) Traité des mal. des enfans, p. 366.

(2) Lib. de affection. num. I 8. Nous rapporterons ici fes termes: Tertiane febris, cum apprehenderit, $f$ quiden, 3'idebitur tibi non purgatus efè quartâ die pharmacum dato: Si vero pharmaco non videbitur opus habere, medicamenta in potu exhibito, quibus febris aut tranfmutetur, ous deficiat. Ce précepte pout-il être plus clair? 
dans les fièvres rémittentes. Iny!

Celle de la fière cst la plus urgente et doit l'emporter. Craindra-t-on que le quinquinan'entraine des suites désagréables. Mais cet inconvénient ne sauroit être comparé au darger inévitable et pressant que le moment présente. Peut-on re douter une rechute lorsquitu est question de traiter une maladie mortalle? Peut-on fure ent trevoir de légères obstructions, très-guérissables d'ailleurs, pour un malade quise trouve en danger de mort? Dans des instants, où le moindre céla peut tirer à conséquence, l'homme instruit doit en profiter, et par une prompte administration du spécifique, il doir arracher une victime au trépas, et donner à lart un tromphe de plus.

Telle a éré la conduite de tant d'observateurs éclairés et judicieux, qui nous ont frayé la rolute, et cherché à nous autoriser de leurs exemples. Leurs succès ont été si constans, si soutenus, si complets, quils 'n'ont pas craint de nous dire que, par la vertu sédative du quinquina, le calme revient plus rapidement que l'orage ne s'est annoncé; que tous les symptômes cessent dès le moment que le fébrifuge a pu se porter en quantité suffisante dans le sang; et que, dans le cas où la fièvre ne s'éteint pas totalement, les exacerbations qui suivent, sont très-peu de choses. Le fébrifuge, pris sur leur déclin en moindre dose, en anéantit la cause sans retour. 


\section{De l'usage du Quinquina}

Prouvons par une observation la réalité de ces promesses.

Un sexagenaire ( 1 ), homme replet et fort en apparence, eut pendant plusieurs jours de légers frissons de temps en temps, des lassitudes - spontanées, du défaut d’appétit, des nausées, des vomissemens quil furent suivis d'une fière qui s'établit par un frisson marqué, par la soif et ensuite par la sueur. Après ce premier accès il y cut beaucoup d'allégement; la fièvre néanmoins continua, avec moins de force à la vérité, et ne se termina pas par un sommeil paisible, et par cette apyrexie qui paroît être le signe distinctif ou caractéristique des fièvres intermittentes légitimes. Le second jour l'accès reparut avec plus de violence et fut moins long: il y eut nausées, vomissemens, grande gêne et douleur dans la respiration, expectoration de crachats mêlés de sang, soif ardente, disparates. Le troisième jour fut moins orageux et assez semblable au premier, si lon en excepte le frisson qui, dès ce jour, disparut pour ne plus se faire sentir dans les accès suivans. Le paroxisme du quatrième jour réveilla tous les

(I) Obfervation communiquée par M. Merlin; Médecin à Lille, \&z dont fon père eft le fujet. Voy. journ. dé Méd. t. XII. p. I 2 g. 
dans les fièvres rémitientes. symptômes du second, mais avec plus de violence; le délire fut décidé, quoique de temps en temps le malade s'en aperçît; le visage et le cou étoient rouges et enflés; les yeux allumés; l'accablement extrême; la respiration laborieuse; le pouls ne correspondoit pas a la véhémence des symptômes: car dans les accès, il n'avoit ni plus de force, ni plus de volume que celui d'un homme en santé, et dans les moindres exacerbations, il étoit très-petit et très-foible : les urines de ce jour et des précédens étoient hautes en couleur, sans sédiment; point de sommeil, mais un assoupissement comateux, avec embarras dans la gorge; le cinquième jour ne fut pas aussi favorable qu'on devoit s'y attendre, d'après la marche de la fièvre en double tierce, et le cas devenoit très-pressant: On avoit placé un purgatif après la troisième exacerbation, et il fut décidé par plusieurs médecins rassemblés, d'employer une forte infusion de quinquina dans le vin blanc, qui, à la dose d'une pinte, fit disparoître entièrement la fièvre; le malade en continua l'usage pendant quelques jours, et la santé fut parfaite. On n'eut besoin que d'un doux purgatif pour achever de nettoyer les premières voies et favoriser l'écoulement des hémorroïdes, qu'une nature active décida chez un homme qui en avoit souvent été attaqué. 


\section{De l'usage du Quinquina}

La guérison de cette maladie, dont le caractère nétioit point équivoque, est sur-tout remarquable par l'effet prompt et notable du quinquina, qui fut tel, qu'on n'eut presque rien à faire dans un sujet en qui la maladie sembloit devoir laisser dessuites à combattre. Qu'on dise, après celia, que dans toutes les fievres rémittentes simples ou bien légitimes, le danger ne dépend pas du dépôt du levain fébrile sur un ou plusieurs organes essentiels à la vie; et qu'en vertı de cette indication, le plus prompt emploi dis quinquina ne soit pas strictement nécessaire!

$\left(^{*}\right)$ Voici un nouvel exemple de l'activité de ce fébrifuge dans un cas beaucoup plus grave encore, et pour ainsi dire désespéré.

M. Gautier, homme de 50 ans, d'une constitution forte, mais dont les humeurs étoient infectées d'une acrimonie dartreuse, fut pris du frisson le septième jour qui suivit son exposition aux influences dangereuses de l'air marécageux. Ce frisson fut suivi par la chaleur que la sueur termina; et cette succession des phénomènes constitutifs d'une fièvre intermittente ent dix-sepi heures de durée. Le lendemain le malade fut bien, et à peine tint-il compte d'une courte et légère exacerbation. Le troisième jour laccès fur semblable au premier, mais, et un peu moins fort, et un peu moins long. Le mat 
dans les fièvres rémittentes.

lade fut émétisé le quatrième de la maladie, et le reste du jour se passa dans l'apyrexie. Le quatrième paroxisme correspondit à ceux du premier et du troisième; il survint le cinquième jour, et fut très-grave; il y cut délire, aucquel succéda un assoupissement qui sembloit îenir du coma; ce redoublement débuta sans frisson, on n'aperçut qu'un simple refroidissement des exirémités; il dura vingt-deux heures, et finit par des moiteurs partielles. Le malade fut très-gai le jour suivant: on profita de ce moment pour placer un purgatif. Il évacua suffisamment. Le sept, un redoublement semblable à celui du cinq, dura trente heures, et sur sa fin il y eut quelques légères syncopes. On donna dans l'intervalle le quinquina, uni à un lavage purgatif. Le huit, jour intercalaire, il survint un paroxisme court, mais orageux; la langue étoit sèche, noirâtre, et les soubresauts des tendons se mirent de la partie. Le redoublement du neuf fut moins fâcheux que celui du sept, auquel il correspondoit; mais l'exacerbation du dix, qui devoit répondre à celle cu huit, fut presque aussi violente que la reprise du sept; dès-lors les intervalles des redoublemens furent courts, le malade restoit affaissé, et le paroxisme étoit divisé par deux périodes; le premier étoit marqué par un délire paisible et soutenu, qui duroit de huit ì dix 


\section{$\$ 76$ De l'usage du Quinquina}

heures; le second étoit caractérisé par un assoù pissement carotique qui duroit autant; ainsi lè malade restoit seize à vingt heures sans connoissance. On appliqua deux vésicatoires aux jambes; le malade faisoit usage de tisanes aiguisées, et de quinquina purgatif; quoique le redoublement des jours pairs fût devenu moins considérable, celui des jours impairs ne perdoit rien de son intensité.

M. Fouquet, célèbre praticien de Montpellier; ayant été appelé, les laxatifs et le quinquina à plus forte dose furent administrés et continués sous la direction du médecin ordinaire. La métastase du levain dartreux, le vice des premières voies, ou la lésion de quelque viscère empêchèrent-ils de placer le quinquina à une dose requise pour s'opposer au retour d'un nouvel accès qui pouvoit être mortel ? Ces circonstances n'eurent pas lieu, ou du moins le péril imminent forma une considération majeure, lorsque je fus rendu auprès du malade. Il étoit dans le cours du vingt-sixième redoublement; c'étoit le fort paroxisme, et il fallut flotter pendant dix-sepe heures entre la crainte de la mort, ou l'espoir que cette exacerbation ne seroit pas la dernière. Le málade prenoit très-peu, et à peine fut-il possible de tirer parti de son état, pour lui faire avaler de temps en temps une cuillerée d'une mixture 
dans les fières rémittentes.

mixture huileuse et fortement camphrée. Après le redoublement, qui parut abrégé de trois heures, le malade reprit ses sens, il se plaignit d'anéantissement et de douleurs aux extrémités; son pouls, qui, dans les forts paroxismes, étoit nature quant à la fréquence, mais petit, mou, foible, prit un peu plus de consistance et d'irrégularité. Je proposai le quinquina à grande dose; pour cet effet, je conseillai de faire une décoction avec une once de bon quinquina, sur cinq verres d'eau, et de délayer dans le premier verre; qu'on donna sur l'heure, trois drachmes de quinquina en poudre; de réitérer de deux en deux heures la décoction fébrifuge, dans le second verre de laquelle on mettroit deux drachmes de quinquina en substance; enfin, de continuer dans les intervalles la mixture camphrée. Ce plan fut exécuté avec un tel succès, qu'il ne survint plis de redoublemens, et que le malade entra dès lors en convalescence.

Elle fut longue et laborieuse, mais sans rechûte: la plaie des vésicatoires eut beaucoup de peine à se fermer, et la foiblesse des jambes fut un symptôme inquiétant. L'appétit se rétablit trèslentement, et ce qui contribua beaucoup à le rappeler et à fortifier la digestion, fut l'usage d'un vin de quinquina, uni à l'absinthe et aux 


\section{De l'usage du Quinquina}

martiaux, ainsi que celui de l'élixir ćoux de vitriol de la pharmacopée de Londres.

Cette observation est frappante sans doute; elle indique le danger de différer le quinquina sur des indications spécieuses, et l'utilité de ce fébrifuge d̀ des époques où il semble que l'expectation a dû le rendre inutile.

LXXVI. On auroit tort cependant, et nous devons le remarquer ici, de s'attendre dans toutes les circonstances à des effets si promptement heureux de l'usage du quinquina. Dans plusieurs cas de fièvres rémittentes, ce fébriftige semble n'agir que par gradation; son énergie spécifique paroît se borner à modérer d'abord la marche de Ia fièvre, ensuite à supprimer un des redoublemens alternatifs quand la maladie marche en double tierce; mais en continuant l'usage du remède, on acheve d'emporter la fievre. Cette opération lente et comme partielle du quinquiná ne détruit point la légitimité de l'indication. Les accidens qu'on veut combattre avec le spécifique, étant réputés dériver directement de la fièvre, ce remède qu'on emploie avant les signes de coction et uniquement pour guérir, avec la fièvre, tous les épiphénomènes qui en découlent, doit aussi être réputé nécessaire en vertu de lindication majeure de la maladie.

M. Soulages, père, s'alita le 2 I septembre 1782 . 
dans les fièzres rémittentes.

Depuis quelque temps il se plaignoit d'une exis tence pénible et de rêver toutes les nuits d'obje très-disgracieux. Au cinquième paroxisme, il survint une attaque de convulsions très-alarmante qui laissa une paralysie de la langue; je fus appelé ce jour là. M. Soulages étoit âgé d'environ 66 ans; sa qualité de Bourgeois l'ćloignant des affaires, et naturellement actif, il prenoit plaisir à diriger quelques travaux sur le bord des étangs oì il passoit souvent les semaines entières. La maladie avoit commencé à l'instar des fièvres catarrhales; mais, dès le début, on avoit remarqué un affaissement particulier, et le malade s'étoit plaint d'une douleur soutenue dans les reins. Les redoublemens quotidiens marchoient en double tierce, et leur intensité se correspondoit à jours alternatifs. M. Soulages avoit déjà été vidé par l'émétique et le surlendemain par un purgatif dont les effets furent soutenus par des mixtures cordiales. Mon premier soin fut de faire appliquer une ventouse à la nuque et donner des frictions rudes aux extrémités inférieures. La raison de la préference que je donnois à ces moyens, trop négligés peut-être, sur les vésicatoires, fut fondée sur le pouls, qui, lorsqu'il est mauvais, contrindique ces sortes de stimulus, de peur que, après l'excitation des forces dues à l'irritation, latonie ne soit infiniment plus grande et plus 


\section{De l'usage du Quinquina}

formidable. On continua les potions cordiales animées par l'alcohol de potasse, ou lilium de paracelse ( qui n'étant qu'un esprit de vin, privé de toute partie métallique, et ảont la portion éthétrée est décomposée et rendue huileuse, peut être administrée moins timidement qu'on ne le fait), et par l'acétite ammonical ou esprit de mindererus, dont les vertus antiseptiques s'approprient très-bien aux fièvres anomales. La tisanne fut une décoction de camomille romaine, adoucie avec le miel, le ventre étant fermé. A ma visite du lendemain matin, la paralysie de la langue n'existoit plus, le redoublement étoit tombé sur les quatre heures du matin, et dans la remission, le malade avoit l'air étonné, du penchant à dormir; son pouls étoit lent et irrégulier sans foiblesse. On appliqua dès lors les vésicatoires, dans la vue de combattre l'état visqueux des fluides, qui, d'après les observations de Lancisi et de Pujati, prédomine dans les fièvres rémittentes des pays simplement marécageux. L'exacerbation qui suivit et qui commença sur les neuf heures du matin, fut seulement remarquable par des disparates et une certaine difficulté à s'exprimer. Le septième paroxisme, qui correspondoit au cinquième et dont le début eut lieu sur les dix heures du matin, amena de nouveau l'attaque de convulsions et la paralysie de la langue 
dans les fièvres rémittentes:

avec beaucoup d'affaissement. Il n'en falloit pas davantage pour déterminer l'usage du quinquina à haute dose. Le malade en prit trois drachmes en substance de deux en deux heures, associé à trois grains de carbonate ammoniacal dans un véhicule composé de vin et d'eat. Ses heureux effets furent marqués d'abord par la disparition de l'affection paralytique et convulsive, ensuite par la moindreintensité du plus grave redoublement. Obligéde varier la forme de l'administration du quinquina, jeus recours à la teinture fébrifuge d'huxham, comme mieux appropriée à la débilité respective et à la putridité dominante dans les suites des fièvres malignes; et ce médicament termina cette maladie d'un aussi mauvais caractère, quoique l'âge du malade et la malignité des accidens ne permissent pas de l'espérer; les selles furent avantageusement sollicitées sur le déclin de la fièvre, dont la durée totale fut de trente-cinq jours. Le malade prit trois onces de quinquina en substance et deux onces sous la forme de teinture. Il ne consomma que quinze grains de carbonate ammoniacal, parce qu'on en discontinua l'usage après la cessation des accidens qui l'avoient réclamé.

Cette observation, qui nous donne lexemple d'une fièvre rémittente maligne, non seulement met au grand jour l'effet graduel du quinquina, 


\section{$182 \quad$ De Lusage du Quinquina}

mais encore slle confrme cette règle du pronostic, que dans les fièvres doubles tierces, dont les redoublenens sont ordinairement inégaux pour la violence et pour la durée, le pronostic doit s'appuyer sur l'observation des redoublemens qui correspondent de deux jours l'un. Il pourroit être très-fautif, si on l'établissoit seulement our la comparaison d'un paroxisme avec celui qui l'a immédiatement précédé.

LXXVII. Dès que les fièvres rémittentes du troisième ordre ne diffèrent que du plus ou du moins des véritables fièvres continues, et que le quinquina n'est point, indiqué dans ces dernières, comme febrifuge, il s'ensuit qu'au lieu de placer dans ce médicament une confiance qui seroit démentie par les effets, il faut adopter le traitement qui convient aux fièvres continues, et, sous ce point de vue, préférer au quinquina, dont on peut cependant se servir quelquefois en qualité de tonique et d'antiseptique, les ćvacuans par haut et par bas, les acides, sur-tout les minéraux, les vésicatoires appliqués de bonne heure, sans quoi l'on perd souvent les avantages qu'on peut en retirer; enfin les cordiaux, les anti-putrides, les diaphorétiques-antiseptiques; remèdes qu'on fait succéder les uns aux autres, et dont on seconde réciproquement les effets, suivant qu'ils sont indiqués par la 
dans les fièvies rémittentes.

nature de la fièvre et par celle des accidens qui l'accompagnent. Le quinquina, comme fébrifuge, et quelquefois même comme antiseptique, putproduire de funestes résultats, parce quilil n'a pas la propriété de changer et d'évacuer les humeurs, ce qui forme en général le point capital de ces maladies; parce qu'en agissant par sa vertu propre et directe, il empêche le développement nécessaire de la fiève pour sa solution la plus avantageuse; parce qu'en portant, par sa faculté tonique, l'érétisme des solides, et le mouvement fermentatif des fluides, à l'excès, il accélère la perte totale du ressort des uns, et précipite la décomposition, la dissolution des autres; parce qu'enfin, en opérant par sa qualité astringente, il supprime, ainsi que l'a très-bien vu Quarin (I), l'excrétion d'une humeur âcre qui, dans quelquesunes de ces maladies, s'est séparée du sahg, et qui aborde successivement dans les premières voies oì le moindre séjour risque d'occasionner l'inflammation des parties dans lesquelles l'humeur s'arrête on se dépose.

C'est pour avoir été administré contre des considérations aussi décisives, que le quinquina a produit, dans quelques circonstances, des effets

(s) Method. medend. febrium, p. 44. 
alarmans et morteis. M. Darluc (I) les a observés dans une fievre rémittente putride, qui fut épidémiq̨ue en Provence, en 1748 ; et M. Vaftapani (2) en fut si frappé pendant le's maladies de Coni et de Turin, dont il nous a décrit l'histoire, qu'il en fut induit à porter sur vefficacité da quinquina un jugement un peu trop défavorable.

LXXVIII. A quelque époque des fièvres rémittentes que se présente l'indication de placer le quinquina, l'expérience nous enseigne que ce fébrifuge ne réussit jamais mieux que lorsqu'il est administré pendant la rémission, et qüil est pris à une distance très-éloignée du prochain paroxisme. La raison de ce phénomène est que I: quinquina n'agit que prophylactiquement contre l'exacerbation future, et que, pour que son effet ne manque point, il lui faut in intervalle sufisant (3). Cette observation limite l'ins-

(I) Journ. de Méd., t. VI, p. 73.

(2) De china-china in Synochis putridis animadversones, \&xe.

(3) Il y a trois opinions concernant le période où il faut adminiftrer ce fébrifuge. Les uns prétendent qu'on doit le faire preridre immédiatement avant le paroxifme; flon les autres, il convient de le donner dès que le redoublement eft fini; il y en a cnfin qui veulent qu'on ly emploie pendant lintervalle des paroxifmes. Cette 

tant de son activité et celui de son administration; elle détruit les assertions mal fondées de ceux qui prescrivent de lonner cette écorce

dernière méthode a été la plus génćralcment fuivie, \& paroit mériter la préférence, parce qu'elle comprend les deux autres; mais fuivant $M$. Home (clinical experimentz, \&c., fect. I) ne font-ce pas peut-ôtre exchufivement les premières on les demières dofes qui opèrent la guérifon? \& dans ce cas, les autres ne font-elles pas, firon nuifibles, du moins inutiles ? Pour décider ce point controverfé, M. Horne a fait io expériences, dont le réfultat cft, Io. que le quinquina of d'un ufage plus efficace \& plus sûr, loríqu'on le donne à la fin de l'accès, ou 40 heures avant le retour du paroxifme, que $f$ on l'adminiftre deux, trois ou quatre heures avant l'accès. Huit des malades, choifis pour les expériences, ont avalé l'ferce du Pérou, immédiatement avant le frifon, fans que la fière air été emportée ; cinq l'on prife à la fin du paroxifne, \& le fuccès a été complet. Sa dofe, dans toutes les expériences, a été d'une demi-once, excepté dans deux cas où elle n'a point paffé deux drachmes.

M. Home obferve, $2^{\circ}$. que fi l'on donne le aninquint peu d'heures avant le paroxifme, la fiève acquicrt plus de force; deux malades ont même eu des vomiffemens; il n'en a pas été ainfi dans le cas où ce rcmède a été pris après la fin de laccès. M. Home remarque, $3^{\circ}$. que comme le quinquina, lorfqu'on le fait prendre à une grande diftance de la fièvre, réuffit mieux, il s'en fuit qu'il faut un temps confidérable pour fon adion." Dans trois malades qui l'ont pris immédiatement avant. 


\section{De l'ufage du Quinquina}

peu avant la reprise, au cominencement dut froid, au plus haut degré de la chaleur. Et quel temps pour placer un remède astringent, que celui où le corps est universellement resserré par le spasme? Quel temps pour placer un remède chaud, que celui où le corps éprouve une ardeur plus ou moins vive ? La raison, l'expérience, indiquent qu'on donne bien plus sagement un remède tout à la fois astringent, tonique et chaud, lorsqu'après le paroxisme les

l'accès, le paroxifme n’a pas été fufpendu, mais le retour du fuivant a été arrêté. On peut faire tous les jours la même obfervation fur les malades qui prennent le quinquina pendant tout le temps de l'intermittence. Il a fupprimé le fecond accès, quoiqu'il n’ait pas arxêté le premier. La quatrième conféquence cure $\mathrm{M}$. Home rire de fes effais, eft que la longueur du temps qu'il faut à l'écorce du Pérou pour exercer fon activité, paroit prouver que l'action de ce remède ne fe borne pas à l'eftomac \& $\&$ au fyltème nerveux, mais qu'il faut qu'il entre dans le fy ftème vafculaire, \& c'eft-là qu'il agit avee le plus d'efficacité. Le fuccès que ce fébrifuge a eu lorsqu'on en a fait ufage, en forme de bains ou de fachet, portent bien à conclure que fon action topique fur l'eftomac, n'eft point néceffaire.

M. Home obferve enfin, d'après ces expériences, que les effets du quinquina donné à la fí de l'accès, font en apparence les mêsnes dans les fièvres quotidiennes \& dans les fièvres tierces, \& qu'il ne faut que is à I6 heures à ect écorce, pour preduire tous fon effet. 
fibres sont trop lâches, lorsque tout le corps est dans l'atonie, lorsque le malade est assez. abattu, assez énervé, pour avoir besoin d'un fortifiant énergique.

LXXIX. Mais comment se conduire dans ces maladies fâcheuses où le type est irrégulier, et où, pour ainsi dire, les reprises se confondent? Les uns (Cleghorn, Werlhof, etc.) veulent, pour placer le quinquina, qu'on épie, avec le plus grand soin, le moment qui sépare les deux reprises: chose qui n'est pas souvent facile. D'autres (Torti, etc.) exigent, que sans attendre la rémission, on donne ce fébrifuge en tout temps. Pour nous, qui ne déférons à l'autorité, qu'autant que l'expérience nous l'a rendue sacrée, nous estimons que le spécifique doit être placé immédiatement après le plus haut degré de l'exacerbation, c'est-c̀-dire, dans le décroissement du paroxisme.

LXXX. La nature des accidens et la marche rapide de la fièvre, doivent décider de la dose à laquelle il faut administrer le médicament. Dans les fièvres très-aiguës des climats chauds, on se voit quelcuefois forcé de prescrire le quinquina sans poids ni mesure, d'en donner autant que l'estomac peut en supporter. Quelques Praticiens même ont cru que dans tous ces cas analogues, on ne sauroit l'ordonner à trop forte 
dose, et le faire entrer par assez de voies. Cleghorn, entr'autres, veut qu'on l'emploie par la bouche, en lavernens, en fomentations. En général cependant, on peut fixer la dose du quinquina, à celle d'une once et demi en substance, et de deux onces en décoction, pour faire prendre dans l'intervalle de deux paroxismes, lorsque le cas est urgent; et comme il est prouvé que les premières doses du quinquina agissent plus spécifiquement que les autres, il convient que la première prise soit très-forte, et que toutes les autres soient diminuées en proportion. Torti faisoit cette première prise de demi-once; nous la faisons ordinairement de trois drachmes, et suivant le précepte de Harvée (I) c'est sur-tout çans les fièvres rémittentes avec diarrhée symptomatique, qu'on se trouve le mieux de donner du quinquina moins souvent, et le prescrire alors en plus fortes doses, sur-tout à la prèmière.

Autant l'on fait du mal en administrant le quinquina dans les fièvres oì ce fébrifuge n'est point indiqué, autant on pêche à donner cette écorce à petites doses dans les cas graves de fièvre rémittente légitime. En effet, de petites doses rendent les exacerbations plus anomales,

(1) Ars fanandi cum expectiat., t. I, p. 30 ze 
dans les fièvres rémittentes.

plus terribles, et suscitent des accidens formidables et mortels. On leur a vu causer des paralysies partielles : effet des crises avortées, d’une fâcheuse métastase ou des aberrations du principe de la vie. Quelquefois même le paroxisme suivant se termine par la mort; trop heureux lorsque ce malheur n'arrive point, et qu'il est permis au Médecin de réparer sa faute.

La veuve Chauvet, femme de $5^{6}$ ans, fut affligée, dans l'automse de 1783 , d'une fièvre rémittente double tierce. Je fus appelé à la septième exacerbation qui avoit débuté la veille à dix heures du soir par un refroidissement très-fort avec une grande diminution de sentiment, de connoissance et de mouvement; ce fâcheux état dura toute la nuit, et s'améliorant progressivement dans la matinée, la rémission fut décidée sur les neuf heures. Alors seulement la malade me montra une langue sale, et demanda pour toute ressource les secours spirituels quilui furent aussitôt accordés. Ce paroxisme étoit un diminutif du sixième, du quatrième et du second, qui avoient toujours commencé sur les quatre heures de l'après midi, et étoient les grandes exacerbations. La première, la troisième et la cinquième, qui avoient toujours débuté sur les dix heures du soir, étoient moins graves et légères lorsqu'on les comparoit aux paroxismes des jours 


\section{De l'usage du Quinquina}

pairs. La malade avoit été purgée deux fois: Frappé du caractère dangereux de cette fière, dont les moindres reprises accuéroient l'intensité des grandes exacerbations, je me hâtai d'employer le quinquina d̀ la dose d'une once en décoction dans une pinte d'eau; et commela langue étoit très-chargée, je mis deux grains et demi de tartre stibié dans la totalité de la décoction précédente, dont la malade devoit boire un verre toutes les deux heures. Je reconnus bientôt que j'avois été trop timide dans l'administration dıı fébrifuge. La grande exacerbation débuta sur les quatre heures de l'après midi par un froid aigu et très-long, la perte de sentiment et de connoissance fut absolue, les lèvres étoient pendantes et livides; la face hippocratique étoit l'emblême de la mort ; les parties chirnues étóient molles. Je m'opposai à la destruction presque totale de la machine par de larges emplâtres vésicatoires, par des frictions rudes sur tout le corps, par des odeurs fortes et volatiles; la malade n'avaloit pas, et les liquides mis dans la bouche ressortoient par les côtés. Tant de symptômes graves n'aboutirent point à la mort. L'indication étoit urgente; auissi fis-je prendre devant moi, demi once de quinquina en substance, dès que ce paroxisme fut tombé, et dans l'intervalle on donna encore pendant trois fois le même 
fëbrifuge à la quantité de deux drachmes par doses, le tout délayé dans une décoction faite avec une once du spécifique. Le neuvième paroxisme fut retardé d'une heure, et eut beaucoup moins d'intensité; dès qu'il fut terminé, on répéta le quinquina de la même manière que le jour précédent, et la dixième exacerbation ne vint pas. La convalescence prit dès lors son commencement, et fut solide. Il est bon néanmoins de faire observer que cette femme rendit pendant longtemps des urines troubles et chargées, et que sa transpiration répandit sensiblement une odeur forte et fétide.

$D_{\text {ans }}$ rette observation, on voit ce qu'on risque de donner le quinquina à trop petite dose. L'Auteur d'une dissertation sur le quinquina, soutenue t Montpellier en 1775 , nous apprend que M. de Barthez a vu une paralysie de la langue et des extrémités inférieures, qui ne put être guérie qu'au bout d'une année du traitement le plus méthodique, et qu'occasionnèrent des doses incomplettes du fébrifuge. Sims a vu des malades en danger de périr par la même cause (I); aussi dans le traitement d'une fièvre rémittente trèsmaligne que cet Auteur observa pendant l'été de I77I, il donna cette écorce à la dose de trois once dans l'espace de vingt heures, ou de cinq

(I) Obfervat. fur les malad. épidém. p. Iss. 


\section{De l'usage du Quinquina}

onces dans moins de trente, sans que l'estomac la rebutât jamais; et M. Colombier (I) ayant à traiter une fièvre très-grave avec des accidens soporeux, administra ce spécifique à la dose de deux onces et demi en quelques heures, savoir, deux onces en décoction, dont la malade buvoit un verre toutes les heures, et demi once en poudre donnée en deux fois. Le quinquina rendu d'abord purgatif, et combiné ensuite avec les martiaux, enlevèrent tous les accidens ultérieurs, et la santé fut parfaite.

Ainsi les Praticiens judicieux savent qu'on ne produit de grands effets qu'avec de grandes doses. Convaincu de cette vérité, M. Clerc (2) vouloit qu'on administrât l'extrait de quinquina, depuis une demi once jusqu'à une once, parce que ce remède ne produit rien à plus petite dose; et pénétré de l'importance de ce précepte qu'il désiroit d'étendre à un plus grand nombre d'objets, cet Observateur avancoit que nous aurions peut-être un plus grand nombre de spécifiques, si nous savions employer les remèdes indiqués, à la dose qui seroit nécessaire. Qu'on recueille ce que les meilleurs écrivains ont laissé sur cet sujet, et on sentira avec quelle justesse

(I) Médec. milit. t. I, p. 3 iо.

(2) Hift. nat. de lhomme malads , t. I, p. 333 .

M. Serrao 
dans les fièvres rémittentes.

M. Serrao disoit qu'il meurt plus de personnes faute d'avoir pris du quinquina, que pour en avoir trop usé; on verra que lorsque ce remède est bien indiqué, on ne sauroit, pour ainsi dire, pécher dans la dose. $D_{e}$ Gorter parle de quel ques malades qui, par dépit ou autrement, avoient pris en une fois la quantité entière de quinquina qu'on leur avoit ordonnée pour plusieurs, et qui, pour prix de leur hardiesse, avoient été, sans le plus léger inconvénient, délivrés de leur fièvre; d'où cet habile praticien conclut que c'est une chose inu-: tile que d'être si timide à déterminer la dose du fébrifuge. Geoffroy a également tonné contre l'insuffisance des doses de ce médicament, au sujet duquel il est bon d'observer que plus les malades en prennent en peu de temps, et moins ils en mettent à en continuer l'usage.

LXXXI. Quoi qu'il en soit, n'oublions pas de remarquer qu'un des effets du quinquina, est d'augmenter, dans quelque cas, les accidens du paroxisme qui suit son exhibition, lorsque ce remède ne l'emporte pas d'emblée. Les praticiens instruits ne s'y méprennent point; ils savent que c'est un indice d'line plus grande liberté dans l'action des vaisseaux sur l'humeur fébrile. Mais combien de jeunes médecins, effrayés par cet orage, abandonnent brusquement l'usage du fébrifuge au moment où quelques doses de 
plus (I) alloient emporter tout le mal! Quils ne s'en laissent donc pas imposer par cette augmentation apparente de la maladie. Qu'ils sachent qu'après avoir employé le quinquina à forte dose, tantôt les deix reprises suivantes se réunissent pour n'en former qu'une tumultieuse, très-longue et propre à effrayer les assistans; mais qu’il survient à la fin une détente générale, tous les couloirs s'ouvrent, et la fièvre est emportée (2); que tantôt le dernier paroxisme,

(i) Dans toutes les maladies où j'ai vu l'ufage du quinquina fuivi de fuccès, dit Sims (obferv. fur les malad. épidem. p. 55, \& p. 156, I58), il paroiffoir d'abord produire des effets défavorables, tous les fymptômes venant à empirer : ainfi, dans les fièvres rémittentes, le paroxifme qui le fuivoit étoit fi violent, qu'il alarmoit ceux qui ñétoient pas familiarifés avec fon opération. Les Médecins ne doivent pas oublier cet effet du quinquina. J'ai vu plufieurs cas, oǹ, pour l'avoir ignore, on difcontinuoit trop tôt ce remède, on fi par bonheur. le malade en avoit pris une quantité fuffifante, on faifoit honneur de la gućrifon qui s'enfuivoit, a quelque chétif remède incapable de l'opérer, tandis qu'on croyoit le quinquina dangereux, dans les cas où il méritoit les plus grauds éloges.

(2) Entre ceux qui ont fait cette remarque, voy. Cleghorn, obfervations on the épidémical difeafes in minorca, \&xc. , p. I9I, ch. III. Sarcone iftoria ragionata de mali offervati in Napoli, t. 1, p. x 80, , \&x. 
dans les fièvres rémittentes:

sans être plus long, est néanmoins très-violent accompagné de délire et cependant suivi de la même crise; enfin que, soit que la maladie soit guérie presque sur le champ, soit qu'elle paroisse résister encore à l'action salutaire du quinquina, cette augmentation des symptômes n'en est pas moins avantageuse, lorsque ce fébrifuge a été placé sur des indications décisives.

LXXXII. S'il y a quelque chose de divin dans la nature des maladies, ainsi que l'a dit Hippocrate, il n'y a pas moins quelque chose de divin dans l'isage et l'administration des remèdes, ainsi que l'a remarqué Richa (I), et ce n'est pas tant du degré d'efficacité des médicamens ou de leur inexplicable manière d'opérer, qu'il faut attendre le plus d'avantages, que de la forme et du temps de leur administration. Nous nous sommes expliqués sur le temps le plus propre à l'emploi du quinquina, et nous aurons lieu d'y revenir encore. Quant à la manière de le donner, quoiqu'elle dépende des circonstances, et qu'on ne puisse conséquemment rien dire de bien précis, nous avancerons cependant que la préparation la plus efficace, et qu'il faut préférer dans tous les cas d'un danger pressant, est le quinquina en poudre, qu'on donne délayée

(1) Conflit. épidem. taurin. dans le $a$ rol. des auvres de Sydenham, p. 382 . 
¿g6 De l'usage du Quinquina

dans une décoction même de quinquina, lors: quil est utile de faire prendre le plus quil est possible de ce fébrifuge, ou réduite en électuaire, au moyen des mucilages qui vallent mieux que les sirops (1), lorsque les malades préfêrent la forme solide. L'extrait de quinquina est ensuite la préparation la plus énergique; on en délaye une quantité à-peu-près égale au quinquina donné en pcudre dans un véhicule convenable: Enfin la décoction de cette écorce, quoiqu'elle soit une préparation moins efficace, et qu'on ait besoin de la donner à une dose plus forte, $n^{\text {'en }}$ est pas moins dolice d'une grande vertu. Des Praticiens éclairés rejettent l'infusion à froid (2),

(I) Voy. Lewis, nouveau defpenfaire, t. I, p. 380.

(2) Les avis font partagés fur l'efficacité de l'infufion à froid. M. Baumí (élém. de pharm. p. 313) affure que le quinquina fournit, dans l'eau froide, toutes fes parties gommeufes, réfineufes \& extractives, \& que la décoetion a cela de défavantageux, qu'elle fait perdre une partie de la réfine. M. Fritze (medechinifche annalem. \&.c.) M. Percival (voy. la note du 9 . CXIV), font de ce fentiment, \& nous favons qu'à Breme on a grande confiance dans un extrait de quinquina, fait par une finple infufion dans l'eau froide, qu'on y appelle anima chince. Cependant Quarin (method. inedend. febr. p. 2 I) s'eft élevé contre toutes ces prétentions. Il a fait répéter fous les yeux, par M. Well, les expériences de $M$. Baumé, \& les réfultars n'ont point été les mêmes; auff 
comme une préparation au moins insufisante. Si la fibre est lâche, le sang dissous, et que l'estomac soit rebuté de tout, on donnera le quinquina en substance; s'il est nécessaire de soutenir quelque excrétion, on choisira l'extrait; et s'il convient de ménager des parties irritables. on usera du fébrifuge en décoction. Les circonstances décideront des secours auxiliaires. L'adj dition du nitre prévient ou remédie à trop dardeur; le camphre augmente la vertu antiseptique du fébrifuge, et soutient la direction des humeurs du centre à la circonférence; le vin exalte sa propriété tonique, et suivant Cleghorn (1), forme le cordial le plus analogue à son effet; la teinture di quinquina renforce le principe fébrifuge et fortifiant; les sels neutres détruisent ce quil peut avoir de mauvais en raison de son astriction, sans compter quils suscitent des évacuations par les urines ou par les selles; le tartre stibié à forte dose (2) en

en a t-il conclu que l'eau froide n'extrait rien moins que les parties folubles du quinquina. Du refte, c'eft une mauvaife méthode de faire bouillir long-temps cetre écorce, lorfqu'on l'adminiftre en décoction, par rapport à la décompolition de la réfine \& la volatilifation du principe fubtil.

(i) Loco citato, p. I94.

(2) De iz ou is grains dans une pinte de décolision 
dirige les effets consécutifs vers la peau; le sucre; en divisant les parties résineuses, rend son opération plus facile et plus prompte, et jamais l'union des acides $(x)$ n'est avantageuse, parce qu'en neutralisant la terre absorbante, ils enlèvent une portion essentielle du médicament; le laudanum l'empêche de se précipiter par les selles, ou de sortir par le vomissement (2); enfin quand on croit devoir préférer le quinquina en décoction ce remède emprunte du vin blanc un surcroît de qualité antiseptique, ot du vin rouge d'une qualité austère une augmentation de vertu tonique et corroborante.

Nous aurions pu parler ici de quelques autres combinaisons que les circonstances peuvent rendre plus précieuses: nous nommerons seulement l'union du quinquina avec la serpentaire de virginie, avec l'écorce d'orange si vantée par

3 de 20 oll 24 grains pour une once de quinquina en poudre. Voy. mém. de la foc. roy.t. III, p. 249, de l'hift. obf. de méd. des hôpit. milit. t. I. p. $187, \&$ journ. de méd. t. XLV, p. 560.

(1) Nous parlons ici de l'indication du quinquina comme fébrifuge \& non comme antifeptique; dans ce dernier cas, l'addition des acides eft pout-être de la plus grande utilité.

(2) Voyez ce qu'a dit, fur cette union du laudanum avec le quinquina, le Docteur Schotte treatife on the Grochus atrabiliofa, \&c. 


\section{dins les fiévres rémittentes.}

Whytt; avec la montarie en poudre recommandée par Aaskou; avec la fleur de camomille suivant le conseil de Baglivi, etc. etc.; et nous passerons sous silence les combinaisons qu'on peut faire de notre fébrifuge avec les martiaux, avec quelques préparations de mercure, etc. etc.

LXXXIII. Nous venons de considérer les fièvres rémittentes dans leur état de plus grande simplicité, c'est-ì-dire, du côté de l'indication dominante que fournit la cause matérielle de la maladie, et pour compléter cette matière, nous nous sommes pareillement expliqués sur totr ce qui concerne le fébrifuge ( $₫$ LXVIl a LXXXII. ) Notre objet est actuellement de discuter les modifications qu'apportent dans l'risge du quinquina, les différens types de ces fièvres, leurs génies, leurs marches, leurs complications, embrassant ainsi dans nos détails successifs tout ce qui concerne ces maladies et l'adrsinistration du spécifique.

LXXXIV. La fière rémittente quotidienne ( $\$$ XVI.) renferme quatre variétés, qui sont la quotidienne et la double quotidienne simple, la quotidienne et la double quotidienne ( $\$ \mathrm{XX}_{\text {, }}$ ) subintrante. Si l'on vouloit une division plus simple, on pourroit adopter celle qui les par$\mathrm{N}_{4}$ 
200

\section{De lusage du Quinquiria}

tage en bénignes et en malignes, et partir de-là pour régler l'adininistration du fébrifuge.

En général dans ces maladies lindication la plus urgente est d'expulser, d'atténuer ces humeurs épaisses qui servent de bouclier au venin fébrile. Si le quinquina étoit alors administré, ne pouvant atteindre le levain de la fièvre, il fixeroit les humeurs grossières et crues qui abondent dans le corps des malades; il s'opposeroit à leur atténuation, il rendroit les crises de ces fièvres plus difficiles, plus tardives, il procureroit ou faciliteroit des congestions dans la plupart des viscères; en un mot, il feroit toujours le plus grand mal, jamais le moindre bien: aussi $L a b b$ veut-il que le quinquina soit souverainement contr'indiqué par la fièvre quotidienne. Ce fébrifuge ne convient donc ici à aucune dose; il s'agit plutôt de donner, le plus promptement 'qu'on le peut, un émétique, par exemple, un mêlange d'ipécacuanha et de tartre stibié, Giu'on place pendant la rémission, le plus piès quil est possible de la fin du paroxisme. L'indication qu'on a pour lors à remplir, étant de pousser, par les principaux couloirs, les sucs glutineux dont les premières voies paroissent être le principal foyer, il s'agit de continuer le tartre stibié qu'on mêle à petite dose dans les tisannes convenables, telles qu'une décoction 


\section{dans los fièvres rémittentes.}

de feuilles de passerage, de jacée, une infusion de fleurs de camomille, etc., ou par les apozèmes faits avec les plantes nitreuses, chicoracées, aiguisées avec quelque préparation d'antimoine ou des sels neutres. Au onzième ols quatorzième jour de la fièvre, on allie le quinquina aux purgatifs; et à mesure que ceuxci deviennent moins nécessaires à cause de la dissipation des humeurs excrémenteuses, on insiste alors sur ce fébrifuge qu'il faut donner en décoction, combiné avec quelque préparation soJuble d'antimoine, et ensuite en substance à laquelle on associe le rob de sureau, le kermes minéral, le soufre doré d'antimoine, les tablettes de Kunkel s'il faut pousser par la peau; avec la rhubarbe, le sel de Glauber, ou le sel polychreste, s'il est utile d'entretenir les selles; enfin, avec le sel d'absinte, la terre foliée de tartre ou l'esprit de mindérerus, si l'on veut augmenter ou soutenir le cours des urines.

Dans les fièvres quotidiennes, graves ou malignes, telies que les subintrantes, ou la cause fébrile est plus âcre, moins embarrassée et plits abondante, ou elle agit sur des liqueurs plus altérées et qui même paroissent avoir quelque tendance à la dissolution. Sous ces rapports, le quinquina est utile et on peut le placer dès le cinquième on sixième jour; on le donne en 
202 De l'usage du Quinquina,

décoction aiguisée avec des sels neutres, avant de l'administrer en substance, si l'augmentation des accidens ou l'opiniâtreté du mal ont rendu cette dernière forme nécessaire; et dans la convalescence on peut retirer de bons effets d'un vin fébrifuge kalibé ( $\mathbf{r}$ ) pour déraciner les derniers effets de la maladie.

LXXXV. La fièvre rémittente tierce comprend la tierce légitime, la tierce illégitime ou fausse, la tierce prolongée qu'on ne peut classer que parmi les rémittentes (2), la double tierce simple, la double tierce subintrante simple, et les différens types de la double tierce doublée. Les unes. et les autres peuvent être bénignes ou d'un mauvais caractère. ( $\mathrm{V}$. les $\$ \mathrm{XVII}, \mathrm{XXI}, \mathrm{XXIV}$.)

Tant que les accidens de la fièvre n'inspirent pas des sijjets fondés de crainte, la tierce n'exige l'usage du quinquina qu'après des signes

(1) Voici la formule d'un vin extemporané, que Quarin a donnée, $8 x$ dont nous avons fouvent éprouvé les fuccès.

Prenez une once $\&$ demi de quinquina groflièrement concaffé, demi once de limaille de fer, decix drachmes de canelle, \& demi once de sucre, mettez dans une bouteille avec une livre $\&$ derai de bon vin. Réfervez $1_{a}$ coulure pour l'ufage qui eft d'en donner deux ou trois fois par jour, deux onces.

(2) Voy. Senac, de recondita febrium, \&ac. p. ss. 
dans les fièzres rémittentes.?

de coction (I) et lorsque le foie ne peut être affecté de la suppression de la maladie. Les indications générales sont, en premier lieu, de combattre, par la saignée, l'érétisme et la phlogose qu'excitent des sucs âcres et bilieux, de détremper long-temps ces mêmes sucs qui menacent d'enflammer le foie. Les purgatifs trop forts sont dangereux; mais il est utile de tenter quelques évacuans qui ne bornent pas leur action aे peser sur l'estomac et se précipiter sans avantage. On retire beaucoup de fruits des feuilles et des follicules de séné, des tamarins, des sels

(1) Le ligne de coction le plus certain , fo trouve dans les urines, \& c'eft là qu'on doit principalement le chercher depuis le ir jufqu'au 2 I. Voyez les préceptes lumineux qu'ont donné, à ce fujet, parmi les modernes, M. Qulefnay (trảité des fièvres, t. I \& II, pafiın.) M. Colombier (médeçine milit. tom. I. p. 2s, 65, 75.) M. Grant (Recherches fur les fièvres, tom. I, pag. $\sigma_{4} \&$ fuiv.) Rega (de urinis ut figno) \& 2. Tous nous enfeignent, que quoique les urines varient en confiftance, en couleur $\&$ mềme en odeur, dans le cours de la maladie, c'eft par elles cependant que l'on s'áflure le mieux que la coction eft prochaine, ou qu'elle eft formée; de forte que leur abondance, ou du moins leur écoulement facile avec un nuage ou $\int u \int p e n f u m$, \& un dépôt muqueux dans les jours critiques, annoncent la correction ou la deftruetion de l'humeur morbifique. Les fignes fournis par les déjections \& l'état des fonetions excrétoires doanent encore beancoup de lumières. 
neutres, auxquels il ne faut pas toujours associer la manne. Le tartre stibié exactement trituré avec quelque sel neutre et de préférence avec le sel marin, réussit , lorsque l'érétisme ( $V$. la note du $\$$. XCIII, p. 238 ) ne s'oppose pas à son administration; on préfere autrement des doses réitérées de crême de tartre unie à la magnésie de sel d'epsom. Si l'érétisme étoit considérable, on se contenteroit d'apozèmes faits avec les plantes chicoracées et nitreusês. Après l'état de la maladie, et les urines donnant des indices de coction, on se permet l'emploi du quinquina dans les potions cathartiques, et l'on passe successivement au fébrifuge "n décoction nitrée et à petite dose, pour accélérer et assurer la convalescence. Si, par l'emploi du spécifique, les utrines deviennent promptement claires; si la tête s'appesantit, et que le pouls ne se développe pas, on auroit tort d'en continuer l'usage; on insisteroit au contraire sur son administration dans les circonstances opposées. Nous préviendrons les jeunes médecins, pour qu'ils ne confondent pas les urines chargées et critiques, avec des urines teintes par la substance colorante du fébrifuge.

Le caractère de la tierce illégitime est trop dépravé, pour ne pas réclamer très-vîte l'emploi du spécifique; mais comme cette fièvre est fort 
dans les fièvres rémittentes:

humorale, il convient de se hâter pour l'usage des évacuans, parce qu'ils peuvent seuls assurer les effets du fébrifuge. C'est dans cette vue qu'on donnera, tant quion le pourra, le quinquina purgatif, et qu'on préférera pour les suites, soit l'extrait du quinguina, soit la décoction animée par le tartre émétique, pour finir par Pusage de la teinture fébrifuge.

Cette méthode rendue plus efficace par une administration plus prompte du quinquina, est la seule qui convienne dans les fièvres tierces malignes, les tierces subintrantes et la tierce prolongée ou hémitritée de Celse. Cette dernière fièvre fut très-commune à Vienne, dans le cours de l'année 1748 ; et Storck (Melchior) qui l'observa, nous apprend que rarement on pouvoit la maîtriser sans le secours de ce précieux spécifique (I).

Comme la double tierce tient pour l'ordinaire le milieu entre toutes les variétés des fièvres tierces rémittentes, son traitement doit être tracé sur la méthode curative qui convient à la

(1) J. M. Storck, Semitertiana, Celfi \& Galeni cortice peruviano curata; in difertatione inaugurali medicâ propofrtâ. Cette differtation eft inférée dans le recueil des thèfes de Vienne par Waßjerberg, t. I, p. 233 , \& dans prelect. ant. de Haen in inflitut. patholog. Bosrhave, to II, dit. in $-4^{\circ}$, pag. 622 , 
variété dont la double tierce suit la marche ou prend le caractère. Nous ajouterons seulement, pour éclaircir le diagnostic de ces maladiés, que lórsque les fiévres tierces ou doublestierces menacent la vie du malade, elles ne sont plus intermittentes, mais rémittentes ( $\mathrm{I}$ ); et qu'il est bien rare qu'il existe des doubles tierces avec des symptômes de putridité et une apyrexie complette (2). Toutes ces fièvres sont d'un catactère très-équivoque; aussi M. Colombier a-t-il dit fort judicieusement, qu'il y a des fièvres qui suivent la marche des intermittentes, quoique le pouls soit continuellement fébrile, en ce que les paroxismes sont marqués en tierce, double tierce, double quarte, etc.; mais quie cos maladies qu'on nomme tierces, doubles tierces, doubles quartes continues, sont presque toujours malignes (3).

LXXXVI. La fièvre rémittente quarte ( $\$$. XVIII ) divisée en simple, double et triple quarte ( $\$$. XXII. XXIII. ) en double et triple quarte doublée ( $\$ . X X V . X X V I$. ), également sous-divisée en bénigne et maligne, présente

(1) Voy. Lautter, hift. bien. morb. rur., p. $147,148$.

(2) Voy. Defcription des épidémies qui ont régné dans la généralité de Paris, 1783 , pag. 62.

(3) Médeciae militaire, tom. II, p. II. 
dans les fièvres rémittentes.

beaucoup plus de contr'indications à l'usage du quinquina, en raison de ces humeurs tenaces et crues qui forme la cause secondaire de la maladie, et qui exigent un long usage de fondans entremêlés de purgatifs et de boissons apéritives. Tel fut du moins le traitement que lon suivit heureusement dans la fièvre rémittente de ce type, qui a été plus commune ici que de coutume après le solstice d'été de 1784 .

Une tisanne apéritive, pour l'ordinaire une forte décoction de racine de chiendent contuse, ou d'orge qu'on animoit avec une certaine quantité de sel polychreste, et qu'on faisoit boire abondamment aux malades, nous conduisoit à l'administration fructueuse de l'émétique, consistant en 15 oll 20 grains d'ipécacuanha et un ou deux grains de tartre stibié. Les vomissemens amenoient des glaires ou des liquides gluans, et lopération étoit terminée par deux ou trois selles de couleur jaune ou verdâtre, de consistance partie liquide, partie poisseuse. La tisanne apéritive étoit répétée le premier jour de la seconde rémission, et le lendemain nous placions l'émétique en lavage. Les malades vomissoient alors une plus grande quantité de bile jaune. M. Tardieu, prêtre, rejeta une véritable humeur attrabilaire, c'est-à-dire, une bile noire, très-glutineuse, et faisant sur les dents 
l'effet d'un acide concentré: ce malade, âgé de 34 ans, étoit naturellement hypocondriaque. Dès la troisième rémission, les malades étoient mis à l'usage des apozèmes faits avec la chicorée, le laitron, la bourrache, la camomille, passés avec forte expression, en ajoutant dans la coulûre, soit le sel polychreste, celui de glauber, le sel ammoniac, ou quelquefois encore l'oximel scillitique. Ces apozèmes donnés trois fois par jour, étoient continués au moins pendant les quatre jours suivans de rémission. On continuoit aussi les tisannes mentionnées, aiguisées avee le tartre stibié, au lieu du sel polychreste; et parvenus au vingtième jour, nous donnions le quinquina à forte dose en substance, trituré avec la magnésie de sel d'epsom et le sel ammoniac ou la rhubarbe, pour prévenir une fièvre quarte intermittente consécutive très-opiniâtre. Lorsque la convalescence étoit décidée, les malades commençoient l'usage du vin médicinal fait avec le quinquina, le fer, le scordium et le vin d'antimoine.

Cette méthode réussit assez généralement. On supprimoit par fois quelques apozèmes, pour placer un' ou deux purgatifs; et lorsque les symptômes étoient graves, ce qui s'observa sur les malades du mois d'octobre, et notamment sur le R. P. Norbert, Capucin, il falloit en 

venir au quinquina dosé, de manière qu'il n'eût qu'une action palliative. En effet, lorsqu'effrayé par les accidens paroxistiques", on administroit largement le fébrifuge, la maladie dégénéroit en double ou triple quarte; l'hypocondre gauche se tendoit, et la fièvre étoit mortelle ou finissoit par une induration de la rate et la cachexie, ou l'hydropisie qui en est si souvent la conséquence. D'un autre côté, lorsqu'arrêté par les malheurs qui stivoient un libéral usage du quinquina on vouloit s'abstenir de cette écorce, la fièvre quarte se changeoit en quotidienne ou continue; avec des épiphénomènes très-fâcheux. Le plus sûr parti étoit alors de se contenter de petites doses du spécifique; elles modéroient la férocité du mal, et permettoient l'emploi des moyens, appropriés à la maladie.

Une pareille observation a été faite par Quarin (I), pendant une épidémie de fièvres tierces rémittentes qui regnèrent à Vienne. Dans les paroxismes de ces fièvres, la bouche étoit amère, la soif urgente, la chaleur considérable, et il y avoit du délire. Les premières voies étant nétoyées, si l'on donnoit le quinquina, cette écorce produisoit une douleur à l'épigastre, de grandes anxiétés, et finissoit par jeter dans la

(i) Metho medend. febr. pag. 24 . 
langueur; mais si l'on négiligeoit l'usage du fébrifuge, la fière dégénéroit bientôt en continue de la plus mauvaise espèce. Pour éviter ces inconvéniens, il falloit débuter par chasser, a l'aide d'un émétique ou d'un purgatif, les impuretés des premières voies, et l'on donnoit ensuite une mixture faite avec le vinaigre, le suc de limon et les sels neutres, à laquelle on ajoutoit deux ou trois drachmes d'extrait de quinquina, immédiatement après le troisième paroxisme. Le quin. quina modéroit la fièvre et l'empêchoit de dégénérer en continue, tandis que les sels neutres entretenoient la liberté du ventre. Dès que la langue n'étoit plus sale, que la couleur des urines étoit moins foncée, et qu'il n'y avoit plus de nausées, on diminuoit la dose des sels, et la fièvre étoit heureusement combattue par le quinquina, dont on administroit d'abord l'extrait et finalement la poudre seule.

LXXXVII. C'est par une suite de la différence qui se trouve dans la cause secondaire et la durée de la rémission, entre les fièvres du type quotidien, tiercenaire et quartenaire, que la dose da quinquina varie dans ces fièvres. Six drachmes ou une once suffisent entre deux paroxismes de la fièvre quotidienne; et la dose entière pour le total des administrations peut être assignée à trois ou quatre onces. On en 
dans les fièvres rémittentes.

donne une once ou dotize drachmes entre deux exacerbations de fièvre tierce, et la dose pour l'entière guérison peut être évaluée à quatre ou cinq onces. Enfin l'intervalle des deux reprises d'une fièvre quarte peut supporter deux onces ou dix-huit drachmes du fébrifuge, et la dose générale peut aller jusqu'a cinq ou six onces, Des observations faites avec beaucoup de soin, ont déterminé que souvent on n'est malheureux ou qu'on n'éprouve aucun succès, que parce qu'on a employé de moindres quantités de cette écorce. Plus la rémission est courte, et plus il faut donner du spécifique en une dose, n'ayant pas le temps de les réitérer. M. Darluc nous apprend que, dans la fièvre rémittente maligne qui désola la Provence en i761, on étoit souvent obligé de conner une once de quinquina en quatre ou cinq heures. M. Darluc parle (I) dı quinquina en substance, et M. Senac propose, quand la rémission est très-courte, de donner la préférence à l'extrait de ce remède (2), dont il croit mal-à-propos qu'un gros a la même efficacité d'une once administrée en poudre. Én effet l'extrait de quinquina doit être donné à presque aussi forte dose qu'en substance.'

(1) Journ. de Médecine, tom. XVI, p. $3 \sigma_{4}$.

(2) De recondita febrium, \&cc. , pag. 408.

$\mathrm{O}_{3}$ 
LXXXVIII. Une observation à placer ici; et qui concerne la classe entière cles fièvres rémittentes, celles sur-tout dont les paroxismes sont doublés, c'est qu'après avoir supprimé le plus fort ou le moindre redoublement, on doit, au lieu de diminuer la dose du fébrifuge, la soutenir ou même l'augmenter pour emporter en entier la maladie. Rien n'annonce mieux ce qu'on peut espérer de ce médicament, que la suppression d'une reprise. Ajoutons encore qu'il faut, après la guérison, donner le plus souvent le quinquina comme prophylactique; et lorsqu'on en a donné de grandes doses, ou qu'on l'a continué long-temps, c'est une pratique trèssage que de conseiller l'usage d'un vin fébrifuge kalibé et antimonié tout ensemble. Nous avons vu. les purgatifís donnés sans indication directe dans la convalescence de certaines fièvres rémittentes, contre lesquelles le quinquina avoit été largement employé, être suivis d'une fièvre intermittente; mais nous croyons cet accident d'autant plus rare dans les fièvres rémittentes, qưil est en général commun dans les fièvres du caractère intermittent.

LXXXIX. Les fièvres hémitritées ( $\$$ XXVII) sont un composé de la fièvre qliotidienne et de la tierce, et leurs causes secondaires, ou du moins leurs complications les plus communes, 
dons les fièvres rémittentes.

consistent dans une diathèse scorbutique du sang et dans la congestion des humeurs viciées et mal digérées dans les premières voies, c'est-ìdire, infiltrées dans le tissu et les organes mésentériques. Cette fièvre, toujours d'un trèsmauvais caractère, et dont la nature tient beaucoup des lypiries et des épiales ( $r$ ), est donc d'un traitement très-difficile; les indications et les contrindications du quinquina y sont également décisives et puissantes. Baglivi, dont lantorité est si grande sur cette matière, se déclare contre l'usage précoce de ce médicament. Il lui reproche de dénaturer la fièvre, de la rendre continue, longue et d'un traitement trèsdifficile, même de la faire dégénérer en hectique. Mead a été de cette opinion. Mais les accidens sont ceux que Baglivi impute à la maladie ellemême, abstraction faite des erreurs commises dass le traitement; et selon toute apparence, ces accidens n'arrivent, lorsque la fièvre n'est pas compliquée, que faute d'avoir omis le quinquina de très-bonne heure, ou de l'avoir administré d'une manière convenable. En effet, linflammation, et, pour mieux s'exprimer, la phlogose des intestins grêles, suivant $S_{\text {pigel, }}$ ou de l'estomac, selon Dodonée, qui est propre

(1) Voy. Senac de recondita febr. p. I 56 .

\section{$\mathrm{O}_{1}$}




\section{De l'usage du Quinquina}

à cette maladie, n'est point la cause du mal ; elle n'en est que le produit; car, dans le cas contraire, comment supposer que la méthode évacuante de Baglivi dans cette fièvre pût réussir une fois seulement; méthode dont Richa a vu linfidélité et le danger, à moins que la congestion r'appartienne qu'au foie ( $r$ ). C'est donc à une dépravation ultérieure des sucs, comme une conséquence naturelle de l'ardeur fébrile, qu'il faut imputer la lésion locale consécutive des intestins, du ventricule ou du mésentère. Or, cette dépravation et son résultat forment tine indication majeure pour avoir recours de bonne heure au quinquina, parce qu'en corrigeant, par la vertu anti-septique de ce remède, la discrasie scorbutique (V. $\$$. CVII) des humeurs, dans le temps que, par la propriété fébrifuge du médicament, on tache de modérer les paroxismes ou d'en supprimer un ou deux des trois qui constituent la période d'une fièvre hémitritée, on remplit une double indication très-urgente et très-caractérisée. Mais eu égard à la cacochylie putride, on soutient l'usage du quinquina par des boissons stibiées, de sorte que le malade doit prendre alternativement une prise de quinquina et un verre de tisanne, faite,

(I) Danss le fecond vọ!, des euv, de Sydenk. p. 47 s. 
dans les fièvres rémittentes.

par exemple, avec l'orge et aiguisée par une suffisante quantiré de tartre émétique. Cette combinaison isolée du quinquina et des doux évacuans, nous paroît préférable, toutes les fois qu'avec une abondance de mauvais sucs, la fièvre présente l'indication principale; elle nous paroît, disons-nous, préférable d̀ la méthode du quinquina purgatif, parce qu'alors on ne donne pas une suffisante quantité de fébrifuge, et qu'en l'administrant, il est trop tôt entrainé par les selles avec les purgatifs auxquels on l'associe.

Tel est, d'après notre propre observation; le moyen de prévenir la fâcheuse tournure des fièvres hémitritées. Une fois reconnues malignes, ces fièvres doivent être combattues par le quinquina, parce que ce fébrifuge est supéricurement indiqué dans les fièves quotidicnnes ( $\$$. LXXXIV) et dans les tierces (\$.LXXXV) d'une nature très-grave et maligne. Aussi ce fébrifuge fut-il employé avec le succès le plus éclatant dans les vraies fièvres hémitritées qui furent épidémiques en plusieurs endroits de l'Autriche. Sur le témoignage de de Haen (I) qui les observa, le quinquina fut administré ciès le cinquième ou le sixième jour, en substance,

(1) Ratio medendi, to V. pag. 173 , t. VI, pag. \$2. $\mathrm{O}_{4}$ 


\section{De l'usage du Quinquina}

aे la dose de six drachmes pour les 24 heures, et la maladie fut jugée, le plus souvent par les sueurs; Au quatorzième ou au dix-sep tième jour, la mort ne fit que deux victimes sur plus de 60 malades. Richa qui vit, en $\mathbf{I}_{72 \mathrm{I}}$, les véritables fièvres mésentériques de Baglivi, ne trouva que le quinquina de souverainement utile, quelque forme que prît la maladie, tant pour en arrêter les progrès, que pour en prévenir les suites fâcheuses. En temporisant, dit ce sage observateur (I), on laissoit à coup-sûr dégénérer la maladie et on facilitoit la dégénération de la maladie en fièvre lente. Enfin, et d'après Werlhof (2), c'est dans la classe des hémitritées qu'il faut ranger ces maladies dans lesqueiles le quinquina eut tant de succès, quoique ceux (3) qui les ont observées, les aient appelées indifféremment hémitritées, pestilentielles, malignes, inflammatoires ou continentes.

On est néanmoins forcé de convenir que le quinquina seroit très-déplacé dans toute fièvre hémitritée assez funeste, assez maligne pour occasionner, dès son invasion dans les en-

(I) Loco citato, p. 478 .

(2) Obf. de febribus, pag. 103 du recueil de fes celle vres in- $4^{\circ}$.

(3) Ajinus, Helretius, Morton, Lancifi, Bianchi, \&ce, 
trailles, une inflammation qui, pour en être lente et incomplette, ne contr'indiqueroit pas moins l'usage du fébrifuge. Cette inflammation se reconnoit, entrautres indices, par la tension et la sensibilité du bas ventre à la pression, par de grandes anxiétés, par une diarrhée séreuse commencée avec la maladie, enfin par les symptômes qui ont coutume de caractériser les fièvres épiales et les lypiries.

XC. Quant aux fièvres continues rémittentes, suivant lidée que nous avons attachée ( $\$$ XXVIII ) à cette dénomination, l'usage du quinquina peut et doit convenir toutes les fois que la fièvre continue n'offie en elle-même rien qui puisse faire proscrire ce fébrifuge. On ne se flatte pas à la vérité de terminer par le même médicament la fièvre rémittente et la continue, mais on espère d'enlever, par le spécifique, les exacerbations de la fièvre rémittente et de décomposer une maladie qu'une telle conjugaison peut rendre très-fâcheuse. C'est ce que nous apprennent les observations de plusieurs praticiens. De Haien (I), voulant traiter une fièvre continue rémittente avec type de double quotidienne, parvint aisément à emporter, au moyen du quinquina, la reprise qui avoit lieu pendant le jour; mais ce fébrifuge ne mordit jamais sur

(I) Ratio medendi , t. VI, p. If. 


\section{I 8 De l'usage Quinquinadu}

le paroxisme nocturne, qui n'étoit sans doute que la recrudescance de la fière continue. Lautter ( $\mathbf{l}$ ), s'étant de même appliqué à guérir avec le quinquina une véritable fière continue rémittente, ne put jamais réussir qu'à dissiper avec ce remède ce que la maladie avoit de rémittent; la fièvre continue poursuivit toujours sa marche ordinaire. Mais ce qu'il est essentiel de faire observer, c'est que, dans ce demi-succès, $l_{a}$ fièvre continue ne fut jamais envénimée par le quinquina; ce qui sufit sans contredit pour nous autoriser à le prescrire : car il n'est pas douteux qu'on ne doive s'attacher, lorsqu'on le peut, à combattre les symptômes dominans des maladies, à plus forte raison lorsque ces symptômes caractérisent une maladie en soi plus ou moins périlleuse. C'est en partant du même principe, que, dès qu'une fièvre en apparence continue se décompose sur la fin en rémittente, on doit ordinairement à ce période se servir du fébrifuge. Telle est la pratique des médecins les plus exercés; et les exemples sont, on ne peut plus communs sur cette matière.

Si l'on veut appliquer le quinquina au traitement des fièvres continues rémittentes avec tout l'avantage qu'on pelit retirer de cette écorce, il fut bien faire attention que ce fébrifinge n'ess

(1) Hiffor. Bien. nwabbor. rural. po $50,51,3570$ 
pas également indiqué et toujours capable de succès dans la fièvre continue rémittente qui est la maladie intercurrente de la fièvre rémittente, et dans la fièvre continue rémittente qui forme lintercurrente de la fièvre continue. Dans ce dernier cas, le quinquina est inutile ou pernicieux; dans le premier, il est salutaire ou indifférent, mais jamais préjudiciable.

XCI. Quelles que soient les indications du quinquina relativement au type ( $\$$. LXXXIV à XC) des fièvres rémittentes, il n'en est pas moins vrai qu'elles sont subordonnées aux indications fournies par le génie de la maladie. Ces dernières indications sont très-différentes entr'elles: on conçoit en effet qu'il doit y avoir une grande diversité entre des maladies d'un génie bilieux $(\$$ XXXII ), putride $(\$ . X X X I)$ ou inflammatoire ( $\$$ XXX).

XCII. Quiconque a bien suivi les effets du quinquina, s'est aperçu que ce remède fait naitre une disposition inflammatoire. De quelie uitilité pourroit-il donc être dans ces maladies, dont le génie tend essentiellement et primitivement à l'inflammation, dans ces cas où il ne faut penser qu’à rafraîchir, qu’à détendre, qu'à combattre des symptômes d'ardeur et d'érétisme? La méthode anti-phlogistique est donc la sevile appropriée, la seule qui puisse convenir. On 
vide les vaisseaux par des saignées suffisantes, et jusqu'à ce que le pouls devienne plus mou (I), on tient le ventre libre avec des lavemens et des purgatifs qui ne puissent point irriter. L'épaississement phlogistique qui reste, sera délayé par une diète rafraîchissante. On relâche les fibres par des vapeurs émolientes et le repos. On adoucit, on calme le genre nerveux par de douces émulsions, avec les sucs légers des fruits mûrs, par une grande tranquillité, et moyennant un air libre, tempéré, et un petit jour. On fait tenir le malade debout, ou du moins assis sur son lit, pendant quelques heures tous les jours, pour précipiter aux parties inférieures le sang qui se porte rapidement à la tête.

(I) Certains praticiens, portés à la faignée aufif aveuglement que quelques Médecins anciens, penfent que, quand le pouls eft grand \& fort, il faut tirer beaucoup de fang! Opinion abfurde. C'eft à la dureté du pouls, à la tenfion de l'artère $\& x$ à la rénitence réciproque du fang \& du vaiffeau, qu'il faut faire une attention particulière. Tant que cet indice est fenfible, il faudra done répandre le fang, pour prévenir les engorgemens \& hâter la coction des humeurs morbifiques; mais il eft dangereux de trop faigner, parce que la fièvre eft néceffaire pour cuire les humeurs \& abréger la maladie. Aufi, la plupart de ceux qui abufent des faignées, fe voyent-ils obligés de recourir de bonne heure aux cordiaux; mais ces moyens ftimulent la nature 8 n’augmentent pas réellemeat la fommẹ dẹ fes forceșo 
On administre enfin tous les médicamens véritablement anti-phlogistiques et qui sont de doux mucilages et les farineux bien délayés, du petit lait de pressure bien clarifié, le sucre, le miel, les fruits mûrs, le nîré, le tout fort délayé; ret l'on ne doit point en employer d'autres que La violence de la maladie ne soit tombée, co que l'on connoît par la chûte des symptômes thumidité des couloirs ou le relâchement des voies excrétoires. Ce n'est que sur de faux perçus et d'après une considération vicieuse; que quelques médecins se permettent l'usage des acides grossiers, des fruits non mûrs, ou de ceux du règne minéral. De semblables moyens précieux dans la fièvre putride d'été, parce qu'il y a dissolution du sang et relâchement des solides, sont très-opposés au génie inflammatoire de la fièvre du printemps, dans laquelle on trouve une contexture ferme du sang tes solides.

La fièvre rémittente au génie inflammatoire, n'est point de celles oì le quinquina puisse être employé immédiatement après les signes de coction, ni pour combattre cette espèce d'affaissement qui suit, pour ainsi dire, la résolution de la période inflammatoire. Cet état demande une diète plus substantielle, et pour lordinaire l'application des vésicatoires sur le 
222 De l'usage du Cuinquina:

déclin du mal. Cette méthode est sur-tout de rigueur, lorsque le cours des excrétions es libre; dans le cas contraire, on se sert du quinquina, comme d'un tonique précieux, et sûr; et la meilleure préparation en est, selon M. Grant (I), la poudre toute simple. On en cesse l'usage aussi-tôt qu'on a rempli ces vues.

Des raisons décisives autorisent ces procédés curatifs. La seconde période des fièvres dont il est ici question, période qu'on pourroit appeler dépuratoire ou purulente, est bien différente de la seconde période des fièvres au génie putride dont nous parlerons ailleurs ( $\mathbb{S}$ XCIII. $\mathrm{n}^{\circ} .2$ ). En effet, pour la guérison des fièvres au génie inflammatoire, la nature convertit la matière phlogistique en matière puriforme; et cette dernière successivement élaborée, doit s'évacuer par degrés, pour qu'il ne se forme aucun dépôt, ou que le sang ne subisse pas une décomposition véritable. La conduite du praticien se borne donc à résoudre, par un régime antiphlogistique et des laxatifs, l'épaississement phlogistique du premier période, et à soutenir, par un régime doucement fortifiant, le travail suppuratoire du second. Il faut même, dans ce dernier cas, laisser le ventre se resserrer, et cependant délayer abondamment, à moins gue

(I) Recherches fur les fièvres, tom. I, p. :326. 
le foie n'ait été particulièrement affecté; car alors, il est bon de continuer quelque temps les purgatifs pour nettoyer ce viscère. Échauffer dans le premier période et rafraîchir dans le second, seroit une pratique également fâcheuse et dégénérative. Mais le quinquina, quoique le moins échauffant et le meilleur restaurant de tous les cordiaux, ne peut néanmoins pas convenir par la raison même qu'il est fébrifuge et astringent. Bien plus, la décomposition de la fièvre en intermittente n'est point encore un prétexte d'y recourir. Sydenham, qui conseille alors ce remède, veut qu'on ne le fasse prendre que pendant l'intervalle de trois accès consécutifs, après lesquels il dit de laisser 14 jours d'expectation pour la dépuration que le quinquina doit aider; aveu tacite, que cet observateur ne s'étoit pas convaincu que ce fébrifuge est foncièrement requis par le génie de la maladie. Le précepte de M. Grant (i) est formel sur ce point; il croit que le spécifique est pour lors la cause d'autres maladies d'une conséquence peut-être plus mauvaise que la maladie originale, et plus difficiles à guérir. M. Retr (2), qui a vérifié à Rochefort, les observations que

(1) Recherches fur les fièvres, t. I, p. 189,3250

(3) Précis d'obfervation fur les épidémies de Roch. fort, pag, II4. 


\section{De l'tisage dul Quinquina}

Sydenham (I) avoit faites à Londres, nous ap: prend que le moyen d'éterniser la plupart de ces fièvres, et souvent de les rendre beaucoup plis dangereuses, c'est de les attaquer, et d'insister sur le fébrifuge. Enfin, M. Tournay, professeur de Nancy, ayant vu dans des cas analogues, l'usage du quinquina être suivi d'une métastasé sur le poumon et d'une vomique purulente mortelle, en conclut que ce remède est trèsdéplacé sur-tout dans certaines fièvres intermittentes qui sont les crises des fièvres aiguës (2).

$S i$ l'on ne distinguoit pas les fièvres rémittentes, dont nous venons de parler, de celles où le caractère véritablement inflammatoire, fait bientôt place à une dissolution putride, on n'auroit que des vues étroites et insuffisantes sur l'emploi du quinquina. En effet, ce fébrifuge est essentiellement indiqué dans les maladies de cette dernière nature, dès que, par des saignées répétées et un régime anti-phlogistique, on a totalement détruit le génie inflammatoire et que l'indication est de s'opposer avec vigueur aux progrès de la dissolution putride. Nous donnerons, pour preuve de ces vérités, l'exemple le la fièvre rémittente qui fut épidémique à Sannat en Bourbonnois au mois de mai $177 \mathrm{I}$,

(3) Euvres de Sydenham, pag. 66 de la trad.

(4) An febribus internittentibus indifcriminatim kinakina? 
dans les fièvies rémitrentes.

êt dont le caractère fut inflanimatoire chez la plupart des malades. Tant que ce caractère fut dominant, ce qui étoit annoncé par la dureté du pouls, par les maux de tête violens, par beaucoup de chaleur et d'ardeur sur la peau, par une coënne forte et épaisse qui couvroit le sang, l'on devoit, sans hésiter, recourir aux saignées plus ou moins rapprochées, selon lidiosyncrasie du sujet et la force de l'inflammation; passer de-là aux anti-phlogistiques, aux javemens, aux fomentations appliquées sur les extrémités inférieures, et, sans trop retarder; au quinquina donné à très-forte dose en subs-: tance, plutôt qu'en lavage et en décoction: S'il arrivoit qu'il se fît une éruption, bien loin d'abandonner ce remède, il convenoit au contraire d'insister sur son usage; le malade en étoit bientôt soulagé, et la guérison étoit plus prompte et plus parfaite ( 1 ). Tant il est vrą̊ que tous les symptômes, quels qu'ils soient qui dérivent du génie de la maladie, ne sauroien être mieux combattus que par les secours indiqués par la maladie elle-même.

L'épidémie de Laschendorf en I75\%, et décrite par Lautter (2), étant de la même nature quie

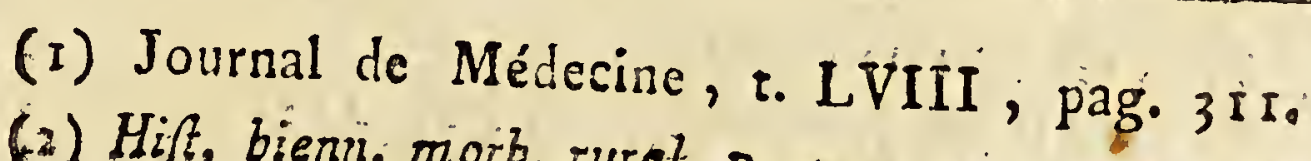

(2) Hif., bientio maib. tural. p. 40, 44,554 , so 


\subsection{De l'usage du Quinquina}

celle de Gannat, fut avantagensement traitée par la même méthode. Richa (1) nous donne unz autre exomple pareil dans l'une des épidémies de Turin; et Senac (2) fait mention d'une épidémie de doubles-tierces rémittentes, qui dégénéroit rapidement en continue, et qu'on ne pouvoit attaquer par les remèdes convenables, sit, au préalable, on n’avoir plasé de cinq à six fortes saiggées. M. Boucher (3) dit exactement ja même chose des fièvres rémittentes doubles tierces, gui régnèrent a Lille en septembre 1764 .

Quoique soumise à l'indication du quinquina, toute fièvre rémittente n'admet plus ce précieux fébrifuge, dès que ses premières impressions établissént, en quelque partie, un noyau d'inflammation plus ou moins forte et permanente. ( V. S. LXX et LXXII ). Cet événement a principalement lieu, lorsque les fièvres rénistentes constituent les intercurrentes des maladies inflam. matoires; tantôt c'est la matrice, tantôt ce soñ les entrailles, l'estomac, le foie, le poumon ou le cerveau qui sont affectés. Il n'importé, toure lésion inflammatoire continue d'un viscère, quoique produit de la fièvre, contr'ndique toujours le quinquiiua, toutes les fois gue ce

(1) Loco citato, pag. 447 .

(2) De recondita febriumi, \&rc. pag. 230.

(3) Journal de Médecine, tom. XXI. pag. 505 . 
dans les fievres rémittentes:

produit sannonce avec la maladie et qu'il né lui est pas subordonné, de manière à suivre ses alternatives de rémission et dé récrudescence. Les inflammations du bas-ventre furent communes a Lille, pendant le mois de janvier 1758 . les nouvelles accouchées et les femmes enceintes y furent sur-tout sujettes; mais elles portoient plutôt l'empreinte des fluxions inflammatoires, que d'inflammations vives : on en vit de compliquées de fievre rémittente, qui, bien loin de demander le quinquina, ne cédèrent qu’à l'emploi alternatif des apozèmes purgatifs et des rémèdes parégoriques, ensuite de plusieurs saignées (I). Parmi les maladies de janvier $176 \mathrm{r}$, la constitution catarrhale étant dominante, on observa des fièvres rémittentes doubles-tierces, dans lesquelles le quinquia ne fut pas favorable d raison de l'engorgement inflammatoire de la poitrine. On fut plus heureux en plaçant quelquefois un émétique après quelques saignées modérées, lors même que la poitrine paroissoir oppressée, et en attendant que la nature soutenue par une diète absorbante et légèrement snti-septique, se ménageât quelque crises qui souventavoient lieu par les urines et par les selles (2); le fond de cette fievre étoit putride

(i) Journal de Méd. t. VIIT, p. 382 .

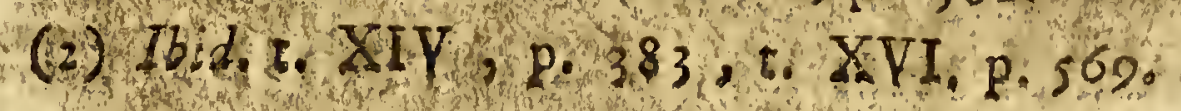

$\mathrm{P}_{2}$ 


\section{De l'usage du Quinquina}

ou tournoit bientôt à la putridité. Nous citerone encore la fièvre rémittente qui fut l'effet d'obstructions inflammatoires dans le foie, marquées par un sentiment de pesanteur ou plutôt de $b a-$ rure à la région épigastrique, s'étendant dans les hypocondres, par des douleurs de ponction, ou des élancemens sourds, à l'hypocondre droit, par quelque élévation ou tumeur de côté, par un teint jaune, etc. Cette fièvre fut observée, lorsque la maladie la plus commune du mois de juin 1760 , étoit une fièvre bilieuse, de la nature des rémittentes, avec des symptômes et une nuance phlogistique; aussi demandoit-elle une méthode anti-phlogistique jusqu'à un certain point, et vit-on que les indications furent mieux remplies par les acides savoneux du règne végétal, l'oximel, les tisannes nitrées, entremêlées de potions absorbantes où entroit la liqueur minérale d'Hoffmann, que par l'emploi du fébrifuge (I). Hasenohrl a produit des exemples d'affection inflammatoire de l'estomac; et d'autres ont présenté des cas de cette même affection dans d'autres parties.

XCIII. Autant les fièvres inflammatoires diffèrent par le génie des fièvres putrides, autant le traitement est dissemblable et opposé dans les indications du quinquina. Dans les rémittentes

(1) Journal de Médecine, t. XIII , p. 287. 
putrides, ce remède est doublement indiqué et par la cause de la maladie et par l'accident ou la putridité. Aussi voyons-nous que c'est dans ces fièvres, où l'on a fait l'usage le plus libéral du quinquina, et où l'on a porté sa dose à un point qu'on ne se permettroit sans doute pas pour toute autre maladie. $S i$ nous considérons quel esí l'état constitutif de la putriditć, nous trouverons que c'est une augmentation de ténuité du sang, jointe à un certain degré d'atonie des forces vitales (1). Or, quel secours est mieux indiqué que celui qui relève puissamment lés forces, qui les somitent, et qui, par cet effet

(I) C'eft à la diverfité d'opinions qu'on a eu fur la fièvre putride, qu'il faut imputer la difcordance qu'on remarque dans la traitement des Auteurs qui ont écrit fur cette inaladie. Les uns ont pris pour fièvre putride, ce qui, dans le fair, n'étoit qu'une fière ftcrcorale; aufi, n'ont-ils vanté que les purgatifs répétés; d'autres l'ont considérée pour ce qu’elle eft réellenent, \& ont profcrit les évacuans trop répétés, parce qu'ils ont vu que ces remèdes angmentoient les accidens, St prow duisoient fur-tout une fonte colliquative très-formidable. Les premiers ont dû profcrire le quinguina; les feconds ont dû l'adopter. Ainfi, les difputes viennent do défác de s'entendre; \& le mauvais emploi du quirguina vient de ce qu'on n'a pas bien faili, qu'on a mal interpréé, \&x en partie mal entendu ce qui conftitue la prirridité, fes divers degrés, fes produits, les complicarions d'mo maladie putride, fes divers états, \&xc. 


\section{De l'usage du Quinquina}

seul, arrête la dégénération ultérieure des liquides que cellii qui, par une action immédiate sur les humeurs, peut établir le mode qui résiste à la putréfaction, contribuer à la dépuration des produits de la fermentation putride, et enrayer les périodes des fermentations spécifiques. vitales du sang et des humeurs dont la rapidité produit et étend la putridité (I) universelle; en un mot, que celui qui opère également avec efficacité et sur les parties solides et sur la masse des fluides du corps animal. Rien ne contr'indique donc le quinquina dans une fièvre véritablement putride, et tout en réclame un libéral emploi. La condition préliminaire pour son administration, est d'avoir évacué les premières voies; et la condition expresse pour son succès est de lordonner à grande dose. Je n'ai jamais. compté, dit M. Sims (2), sur moins de six ou sept onces dans un danger pressant, et données dans environ deux jours; mais quelquefois trois onces sufifisoient; et j'atteste ici n'avoir jamais, vu un cas de fièvre nerveuse, putride ou maIigne, où le malade qui en a pris une quantité requise, ait péri. Jajouterai aussi que je n'ai jamais vu dans ces fièvres l'estomac incommodé

(5) Voy. Baršlzq, nouv. élén. de la fcience de fliomme, t. I, p. Ior \& fuiv.

(2) Obferv: fur les malad épidém. p. 7230 
par la plus forte dose, soit dans le temps de son usage, soit après; et que, lorsque je pouvois engager le malade à prendre les premières doses de quinquina, je le continuois sans interruption, jusqu'à ce qu’il en eût pris une quantić suffisante, me faisant une règle de le donner dans le moindre temps possible, vu que le rebut des malades pour ce remède, naît souvent du trop long-temps qu'on met à le leur faire prendre. Dans des cas analogues, M. Veryst a osé porter la quantité de quinquina bien plus loin, puisqu'il enseigne, dans le traitement des fièvres automnales des pays très-mal sains, d'employer $20,3^{\circ}, 40,50$ et jusqu'à 80 onces de quinquina; se permettant même dans les cas trèsfâcheux, d'en donner 9 à 10 onces dans les trois premiers jours.

Si le quinquina est un remède majeur dans le traitement des fièvres rémittentes putrides, il n'en est pas moins essentiel de solliciter lentement et sans trouble, les évacuations que la nature affecte et qui peuvent expulser les prow duits morbifiques. Souvent cetie demière indication tes long-temps dominante, et pour lors on insiste de préférence sur les secours qui peuvent la remplir. Parmi les moyens propres à soutenir les évacuations alvines, nous distinguons le tartre stibié qu'on administre ì dose 
232

De l'usage du Quinquina

convenable, dans un mélange d'eau et de vin dont les malades doivent faire alors leur boisson ordinaire. Cette eau rougie, suivant l'expression d'un praticien judicicux (I), tient lieu, lorsqu'elle est émétisée, de délayant, de laxatif, d'incisif, de cordial ; elle remplit plusieurs points de la plus grande importance, et ses succès sont, pour l"ordinaire éclatans chez les pauvres gens, dont la constitution est presque toujours épuisée.

La surabondance des maúvais sucs, dont la nature veut constamment se débarrasser par la voie des selles, n'est point, dans les rémittentes putrides, une raison de eraindre l'administration. précoce du quinquina. On combine alors les purgatifs appropriés avec le fébrifuge, et c'est toujours avec un avantage marqué. Nous disons plus, il y a tout lieu de croire qu'on abrégeroit considérablement la durée de ces fièvres, sil'on osoit les atraquer presque d'emblée, avec le quinquina à forte dose, mêlé avec quelque substance purgative, par exemple, la magnésie du sel d'epsom qui parôit avoir la propriété, nous ne disons pas de purger, mais encore de prévenir et de calmer les douleurs que donne quelquefois,

(1) M. Boncerf. Voy. Journal de Médecine, t. LXIII, p. $162 ; 196$ 
dans les fièyres rémittentes:

le quinquina, sur-tout lorsqu'il est pris à grande dose. Cette pratique, qui paroît hardie et qui differe, en effet, très-peu de celle de plusieurs Médecins anglais, a pleinement réussi à un de mes confrères qui pratique dans un pays marécageux. "J'ai appliquéle fébrifuge de M. Lorentz, "nous apprenoit-il par ses lettres, au traitement ») des fièvres putrides, simples ou graves, et jus》) qu'ici je ne puis parler que des bons effets que ग) j'ai obtenus, J'ordonne à ma première visite " que le malade commence l'usage du quinquina " mêlé avec parties égales de magnésie de sel " d'epsom, et jefaisprendre une, deux ou trois " drachmes de ce mélange toutes les heures ou " toutes les deux heures, suivant le cas. J'en " obtiens toujours des évacuations abondantes " par les selles; et les urines sont en général " plus chargées. Jamais il n'en a resulté d'incon"vénient, et le plus souvent l'événement a été " plus flatteur que je ne pouvois l'espérer. Des "maladies qui s'annonçoient très-mal, ont été, "pour ainsi dire, suffoquées; leur guérison a " été l'afraire de quelques jours. Toutes ont paru " avoir un cours plus tranquille es moins orageux " que celles qui n'étoient pas combattues à ma " manière, et leur durée a été moins longue. "Ce qui me fait croire que je ne m'en suis pas " laissé imposer par quelques sticcès accidentels 


\section{De l'usage du Quinquina}

" et fortuits, c'est que j'ai fait mes essais pendant "le règne des fièvres putrides, qui malheureu" sement ont été plus répandues pendant les " étés et les automnes de 1783 et de 1784 . "Ayant eu l'avantage de la comparaison et de " la répétition, jai pu me convaincre que toutes * les fièvres putrides ne résisteroient point au * quinquina purgatif, pourvu qu'or le donne à * forte dose dès les premiers jours de la mala" die; double condition expresse, sans laquelle " le quinquina ne réussit ni aussi pleinement, * ni avec autant de sûreté".

Nous avons dit ( $\$$. XXXI) que, dans plusieurs cas de fièvres putrides, la dissolution di sang qui les constitue telles, étoit précédée par un épaississement muqueux, ou par une densité inflammatoire; et nous avons donné, pour exemple du premier cas, la fièvre muqueuse de Gottingue, et pour exemple du second, la fièvre qui forma la constitution dominante de l'été et de lautomne en $178 \mathbf{r}$, dans la majeure partie de la France. Il nous reste à parler du traitement qui fut employé, pour completter, autant qu'il est en nous, T'histoire des maladies putrides, et montrer ce qu'on peut attendre du quinquina dans les diverses circonstances.

I0. Dans la fiève muqueuse, il existe d’abord un épaississement très-tenace, et qui, dans cette 
maladie, couvre le sang d'une saignée, d'une croûte semblable à celle dulard : cet épaississement, a fort bien dit Cartheuser ( $\mathrm{I}$ ), contracte par sa stagnation continuelle, une corruption putriJagineuse ; les sels mixtes s'alkalisent indifféremment, prennent une nature fort pénétrante, et toute la masse muqueuse se résout en un fluide extrêmement caustique. Les indications de cette fièvre sont donc d'expulser par les vomitifs l'amas de mucosités contenues déjà dans les premières voies, clatténuer, par les sels neutres et les boissons stibiées, l'épaississement muqueux; enfin, de travailler sans relâche à la fonte des sucs tenaces, pour prévenir ou du moins pour adoucir leur passage à la dissolution putride. Parvenus. à cette époque, il est essentiel de continuer les évacuations; mais comme lhumeur morbifique a acquis la plus grande causticité, on ne sauroit employer des minoratifs trop doux. Il convient même souvent d'en émousser l'action par des narcotiques, et d'y joindre les correctifs de la putridité bilieuse, sur-tout les acides minéraux. Telle fut la pratique de Wagler (2), elle fut très-heureuse; mais le quinquina ne fut point oublié, il convenoit trop bien en raison du ca-

(i) De miorbo mucofo, pag. 87

(2) Jbid. pag. $27,30,35,76,155$. 


\section{De l'usage du Quinquina}

ractère rémittent de la maladie, qui dailleurs étoit dégénérée d'une fièvre intermittente. Employé dans le fort de la maladie, pendant la rémission, spécialement lorsqu'il y avoit des sueurs nocturnes ( $\mathrm{I}$ ) ou un dévoutement symptomatique (2), l'extrait de quinquina, auquel on donnoit la préférence, avoit des effets peu communs; it arrêtoit, à l'avantage du malade, les évacuations inutiles et dangereuses; il changeoit la coction, prévenoit lá toưnure gangréneuse, et, en fortifiant doucement le système nerveux et toutes les parties foibles, il facilitoit la crise suppuratoire. Placé sur le déclin de la fièvre, ce même extrait invigoroit les premières voies, il excitoit une douce transpiration, et dissipioit tous les autres produits morbifiques. Sur la fin de la maladie, on faisoit usage avec fruit de la décoction de quinquina, dans laquelle on délayoit quelqu'extrait amer, ou qu'on aiguisoit avec quelque sel neutre, si l'on craignoit ou s'il $y$. avoit de légères obstructions à dissiper ; enfin, dans la convalescence on donnoit le quinquina en substance, uni avec la limaille de fer. Si des symptômes urgens forçcient à l'usage précoce de

(1) Il eft bien rare que, dans les rémittentes putrides, la fueur ne fe déclare pas à la fin de chaque exacerbas tion. Colombier, Médec. milit. t. II , p. II s.

(2) De morbo mucolo, II 4 . 
dans les fièvres rémittentes.

cette écorce, il convenoit, après son action, de revenir aux doux résolutifs, comme étant les remèdes directement appropriés à la dégénération humorale (I).

Toutes les fièvres rémittentes putrides simples des pays marécageux sont une espèce de diminutif de la fièvre muqueuse, dont nous venons de rendre compte. Il faut y combattre un phlegme grossier et crud, suivant les expressions de Lancisi (2); aussi les vésicatoires et le quingquina, qui sont deux spécifiques de ces maladies (3) ne réussissent jamais mieux que lorsque les vésicatoires ont été appliqués dès les premiers jours, pour préparer le succès de l'écorce fébrifuge.

$2^{\circ}$. Quant à la densité inflammatoire qui précède, dans quelques fièvres rémittentes, la dissoIution putride, ce n'est point une contexture ferme dusang et des solides, comme dans les fièvres inflammatoires ( $\$$. XCII. ); c'est un excès d'érétisme auquel se joint l'effet d'une cause trèsâcre, qui commence par épaissir les humeurs avant de les dissoudre: aussi la saignée y est généralement contrindiquée, ou dli moins voit-

(1) Wagler, de morb. mucof.p. 9I, I I 4 , Irog.

(2) De nox. palud. effluv. p. I66, 272.

(3) De nox. palud. effluv. pag. 166. 
${ }_{23} 3$ De l'usage du Quinquina

on qu'elle n'y réussit pas d'une manière sensible? Une dose d'ipécacuanha ou de tartre stibié suffisante pour exciter un vomissement prompt et avec secousses, est, de l'aveu des Médecins, un préliminaire indispensable, puisqu'il s'agit d'évacuer au plutôt une bile surabondante et même dans un état de turgescence. On étend les bienfaits de cette évacuation, en en procurant de nouvelles à l'aice des lavemens multipliés, des laxatifs (I) placés dans les momens de rémittence, ou du tartre stibié, dont on aiguise les boissons lorsqu'on n'en est pas empêché par le degré du spasme (2). Dès que par ces moyens, et

(1) Voy. Radelfel: de evacuantium ufu in febriutri acutarim tam initio quam decurfu.

(2) Les avis des Praticiens ont été partagés fur l'uti: lité du tartre ftibié mêlé à petites dofes dans les boifCons, \& continué pendant plufieurs jours. M. Dazillé (obfervai. fur les maladies des. Négres), s'eft plaint qu'à Paris, comme dans les Colonies, l'on abufe étrangement de cette adminiftration de l'émétique à petites ciofes ; plusieurs Médecins de la faculté de Paris l'ont déclarée dangereufe dans les affemblées des prima menjbs des Is janvier \& 4 février i 78 i (Journal de Misdecine; \&. LVII, p. 274, \& gazette falutaire ann. $1782, n^{\circ}$. XVIII, colon. 3.) M. de Gardaune s'en est de même expliqué dans fon traité des maladies des Créoles en Europe. Cependant des Praticiens du plus grand mérite en on reconnu $\&$ avoué les fuccès. Une pareille diverficé d'opinions fur un point de pratique de la plis 
ceux qui les secondent, tels que les boissons acidulées ou ascécentes, la diette végétale, le nitre camphré, etc, on est parvenu à dissiper l'érétisme, et que la maladie faisant des progrès évidens, ne présente plus que des accidens, d'autant plus inquiétans quils ne sont plus infammatoires, le quinquina devient un remède précieux, et rien ne peut suppléer à ce fébrifuge aussi puissant anti-septique que tonique efficace. Nous lavons toujours employé avec succès, disent les Membres de la société royale, d'après lesquels nous parlons, sur-tout dans deux cas assez ordinaires. Le premier est celui dans les-

grande importance, ne peut provenir que de la prévenrion qu'on a prife pour le tartre ftibié, donné par fracrion, d'après fes effets obfervés dans le càs où ce remède étoit contr'indiqué. Ainf M. Ricquet (méthor de traiter les fiévres putrides \&z vermineuf́es qui règnent depuis plufieurs années dans les environs de Lille), obferva que le tartre ftibié réuffit dans les mêmes maladies à Comines \& à Houplines, mais qu'il ne produifit pas les mêmes effets à Haubourdin \& à Santes. Le tarIre ftibié nuira toujours dans les cas d'érétifme, \& Oorfque la chaleur fera très-ardente. Alors, bien loin de provoquer la dépuration défirée, il augmente les accidens, \& fufpend les évacuations. La chaleur deviont plus âcre, le pouls plus dur \& plus ferré, \& météorifine du ventre plus confidérable. Mais dans les cas contraires, ce reinède fera toujours un bon effet. 


\section{De l'usage du Quinquina}

quels les symptômes inflammatoires n"inspirant plus de frayeur par leur violence, nous voyons l'apathie et l'irrégularité putride prendre la placedes premiers accidens. L'érétisme paroît tombé, mais à sa place les soubresauts dans les tendons, un délire morne et taciturne, une somnolence pénible, la gêne même de la respiration, font craindre une fin prochaine. Souvent à ces accidens se joint une fétidité dans les urines, qui appartient au caractère putride. Le second accident qui nous a forcé la main sur l'usage du fébrifuge, est celui où, après les premières évacuations spontanées, l'estomac reste dans un état de nausée, de dégoût et même de haine pour ce quion lui offre. Les vomissemens de bile porracée ne nous ont point détourné de l'usage de ce remède: alors il nous a paru que l'indication étoit de l'unir à quelque lavage laxatif. En un mot, nous avons vu, dans ces cas effrayans, réussir très-promptement l'usage du quinquina donné à des doses trèshautes, et continué pendant plusieurs jours de suite. Une forte décoction d'une orice dans une livre de liquide ne nous a causé aucun accident. On pourroit dire qu'on voit le quinquina aviver le malade, et donner à la nature de nouvelles forces contre son ennemi. Aussi son union avec les purgatifs est-elle toujours heureuse. Le camphre 
dans les fièvres rémittentes.

camphre (I) nous a paru aussi seconder à merveille sa vertu, sur-tout dan: les cás convulsifi. Enfin, dans les convalescences, lorsqu'il restoit quelques accidens dे détruire, résultats assez constans d'un mauvais régime, on retiroit becucoup de fruit d'un usage long-temps continué du quinquina, combiné avec les mariaux et avec quelcues substances camphrées et volatiles (2) ì doses graduellement diminuées.

Ainsi, dans tous les cas de putridité, le çuinquina a été de la plus grande ressource. Si l'on trouve quelque discordance dans le temps de son emploi et dans les doses, chez les auteurs qui ont traité de l'utilité de ce fébrifuge anti-septique dans les fièvres rémittentes putrides, c'est qu'il y a une infinité de nuances dans la putridi:é, et qu'on n'a pas assez distingué ce qui appartient à la plénitude bilieuse du duodénum et à l'altération putride du sang et des humeurs, ou à un état mixte composé de l'une et de l'autre. Plus il y a pourriture dans les humeurs et flaccidité dans les solides, plus on doit faire fonds sur le quinquina, et plus il faut l'ordonner à

(I) Voy. M. Buechner, de ufucorticis peruviani, cum camphorâ remixti, in febribus ex putredine ortis: Lyfons; an eflay upon the effects of camphire and calomel, Es.: Collin, camphore vires, \&c., \& $\mathrm{x}$.

(2) Voy, Lancisi, de nox. palud. efur., p. 2030 


\subsection{De lufage du Quinquina}

grandes doses; car, comme l'ont très-bien dit Richa (1) et Ramarini (2), le quinquina ne réussit jamais mieux dans les fièvres putrides, que lorsqu'il faut plutôt resserrer que stimuler. C'est alors qu'on peut dire que le quinquina est un des moyens les moins infudeles pour rallier les principes du sang, remonter le ton des solides et favoriser la crise: au lieu que les délayans et les évacuans prodigués dans ces circonstances, dans l'objet de diminuer la matière morbifique, de l'évacuer par tous les couloirs et d'en énerver les foyers, ne préparent que des demi-crises, en troublant les mouvemens de la nature et affoiblissant l'action systaltique des vaisseaux. Ces efrets du quinquina ont été si universellement reconnus, que M. Pellicioni (3) a présenté cette écorce comme le meilleur prophylactique des fièvres aiguës, putrides malignes, dans lesquelles Lettson (4) ne prescrivoit que le grand air, le

(I) Confitut. épidem. taurin. p. 385 , ann. I720, $x>2 z, \delta$. XXXIII \& feq.

(2) De confititition. annorum I690 ̀े I694, in mutineirly civitate in conft. anm. 1690, 6. XLVII, ann. I792, 5. XXXII \& LVI.

(3) Sopra la efficacia è virtu della chinachina, I769.

(4) Médical mémoirs of the général difpenfary in London, \&xc., art. I, \& Banau, moyens propres à combattre les fièrres putricies \& malignes. 
dans les fières rémititentes.

vin, leau et le quinquina à la dose de quatre ou cinq onces par jour. Quand la dissolution putride est considérable, ce qui a lieu lor sque les malades exhalent une forte odeur d'aigre et d'ui1, on peut donner un mélange d’alun et de quinquina. Toutes les boissons, dans les fièvres pu. trides, doivent être donnźes froides.

XCIV. Le traitement des fievres bilicuses, si analogue d'ailleurs à celui des fièvres putricies, du caractere desquelles elles approchent beaticoup, quoiqu'on ne puisse pas les idcntifer, comme l'ont fait Pringle (I) , M. Retr (2) ct autres, a cela de particulier, qu'il faut beaucoup plusinsister sur l'usage des purgatif, que dans toute autre espèce de fièvre, et que la saignée (3)

(1) Obf. fur les maladies des armées, t. I, pag. $300^{\circ}$

(2) Météorologie appliquée à la Médecine , p. 82 .

(3) Ceux qui confultent les auteurs anciens, ont du s'apercevoir que leur autorité eft très-favorable à l'urge de la faignée dans les maladies bilienfes. Mais ce inffrage n'eft d'aucun poids, parce que les maladies que nous appelons inflammatoires, étoient connues chea les anciens fous le nom de bilieufes, à caufe de la croûte phlogiftique \& jaunâtre qui recouvroit le fang; croûte qu'ils appeloient fang bilieux, pituiteux, omon aima. Baillou nomme pleuréfie bilieufe, toutes colles que nous appellerions inflammatoires; \& Hippocrate luimême, dans fon livre des lieux dans l'homme, dome le noin de bile à la pleurćfie. Notre pleuréfic bilieufe eft

$Q_{2}$ 


\section{De l'usage du Eunquina}

y esî en général contr'indiquée, ou du moins n'est nécessaire que par des circonstances étrangères à la maladie. En effet, le foie s'engorge facilement, pour peu que les humeurs morbifiques soient retenues; et la jaunisse, la dyssenterie sont, pendant la fièvre, des produits ordinaires de cette rétention, comme l'engorgement et l'obstruction du foje, l'hydropisie, en sont communément les résultats, après la maladie. Délayer et évacuer constituent done la vraie méthode des fièvres dont le génie est bilieux; les émétiques doux sont bons à réitérer quelquefois, à cause du pouvoir qu'ils ont de dégorger le foie et de secourer tous les viscères. Ensuite moyennant de douces purgations répétées, on épuise le foyer humoral, on abrège la fièvre, on écarte les épiphénomènes qui en dépendent, et on en prévient les suites. Le quinquina n'est

une maladie d'une toute autre efpèce. Aufii, quand nous lifons que Galien prefcrit, dans fes comment. fur l'aphor. 23 , liv. I, qu'il faut faigner jufqu'à la fyncope dans les maladies bilieufes, \& dans les Arabes, qu'on ne fanroir trop redouter de verfer le fang dans ces mêmes fières, il faut convenir que Galien et les Arabes n'ont pas vouli parler de la même maladie, \& que l'un traitoit nos vraies fièvres inflammatoires, tandis que les autres n'avoient en vue que nos vérirables fièvres bilicufes. 
dans les fièvres rémitcutes.'

pas néanmoins à mépriser; il peut soutenit le ton des parties que l'effet des évacuans abat, aider l'action des purgatifs qui n'agiroient autrement qu'avec lenteur, et coopérer airsi pour guérir plutôt et rendre la convalescence plus conite. Cependant il faut ciaindre son cfiet astringent, avoir toujours en vue la nécessité de l'évacuation, et se souvenir du préceptede Baglivi, qui veut quion ne donne jamais le quinquina que le corps n'ait été suffisamment évacué par une méthode sage et dogmatique. Duns les cas simples, le quinquina doit être à-peu-près inuîle: dans les cas graves, il est nécessaire; mais, au. tant qu'il sera possible, cn le placera après quelques signes de coction et uni aux purgatifs: dans les cas plus graves, on ne peut sen passer, sans comprometre les jours du malade. On le donne dans tous les états de la miladie, on purge ensuite à la faveur du répit qu'll procure (I).

Les acides, quoiqu'astringens de leur nature, sont d'une application plus générale, en ce quils émoussent la causticité de la bile, ainji que WGber (2) l'a prouvé. On sait en ontre que cerre

(I) Voy. Bianchi hifioria hepatica, t. I, p. $282, n^{\circ}$. II I. Guidetti, ib. p. G29.

(2) Dans le fecond vol. des préleçons de de Hacn fur les inftituts pathologiques de Boerrhave, t. II. édit. in $-4^{\circ},$, f. 633 . 


\section{De l'usage du Quinquina}

matière est décomposée dans les premières voies par les acides qui, par-là et dans ces circonstances, ont des effets évacuans. On voit donc les avantages que promettent, dans les affections bilieuses, les substances qui réunissent à l'acidité, une vertu minorative; tels sont les tamarins, et sur-tout la crême de tartre que les praticiens judicieux, suivant l'expression de Weber, prescrivent pour l'ordinaire à grande dose.

Cette méthode seroit nuisible et infructueuse dans le traitement de ces fièvres rémittentes bilieuses, oì les humeurs ont acquis un caractère de viscosité plus ou moins considérable. Quels que soient les accidens de ces fièvres, le quinquina ne pelit rien contre eux, s'il n'est uni avec des atténuans énergiques, tels que le sel ammoniac (I) qu'il faut même préférer ou don-

(I) C'eft pour avoir vu réufir compiettement le fel ammoniac dans quelque cas de fièvres rémittentes bịlieufes, qu'on lui a afigné un rang diftingué parmi les antifeptiques. Mais, dit Stoll, on n'a embraffé cette opinion, que parce qu'on a placé la caufe de toures les fièvres automnales dans la diffolution \& la putridité des humeurs. Il en eft paimi ces fièvres qui font canfées par une cacochylie glumenfe, \& le fel ammoniac agit Dlors comme atténuant, \& non comme anti-feptique; ratio med, t. I, p. 79. 
dans les fièvres rémittentes:

ner pendant long-temps avant que d'administrer le fébrifuge. Telle fut la pratique gue suivis Stoll (I) dans les fièvres rémittentes qui régnèrent a Viemne en Autriche, dans le cours de l'année I776. Les malades vomissoient avec beaucoup de peine, et souvent en petite quantité, une humeur jaune, très-gluante, laq̣uelle adhéroit fortement alix parois des tuniqucs intestinales, et engouoit tous les vaisseaux ghirnd elle pouvoit pénétrer dans le genre vasculcux. Pour la chasser, il falloit se servir d'un émétique bien dosé et le réitérer nême par intervalles, lorsque par de bons résolutifs, on avoit rendu les humeurs mobiles. Une mixture faite avec cinq onces d'eau de sureau, une once d'oximel simple, une once de rob de sureau et du sel ammoniac, depuis deux drachmes jusqu'à trois, qu'on faisoit prendre à doses rompues dans les 24 heures, réussissoit dans tous les cas oì la nature vigoureuse se suffisoit pour la coction. Sil étoit bon de laider, soit ia cause de la surabondance des humeurs cu d'une plus grande tenacité, soit parce que les forces avoient été abattues par des saignées déplucées ou d'autres accidens, on avoit recours à la ram cine d'arnica (2) en poudre, remede préférable

(2) Rat. med. t. I, p. 78 , édition de Paris.

(I) Il n'eft point de Médecin qui ignore les expé$\mathrm{Q}_{4}$ 


\section{De l'usage du Quinquina}

au quinquina dont il a quelques propriétés éminentes, dans toutes les fievres de ce genre bilioso-pituiteux ou simplement pituiteux, gastr:ques, mésentéricues, putrides ou malignes, et notamment dans les diarrhées énervantes et opinî̀tres ou dans les fux dyssentériques (I) qui accompggnent ces muladies. Lorsque la fièvre résistoit, presque subjugée par la méthode précédente, on l'emportoit tres-promptement avec le fébrifuge 2 ). Employée plutôt, cette écorce

yiences que Collin a frites, tant avec les fleurs qu'avec la racine de l'arnica; expériences qui prouvent que les fleurs de ce fimple font fébrifuges \& antifeptiques, \& que la racine eft auffin bon anti-feptique, \& fur-tont très-eficace contre la dyssenterie \& la gangrène. Collin guérit. avec les fleurs de l'arnica, p'us sûrement \& fans aucune fuite fàchufe, les fièves intermittentes, qui, dans l'épidénie de 1770 , dégénéroient en fièvres putrides, lorfqu'on les traitoit avec le quinquina. Cepen. dant, quoiqu'il y ait peu de ces fièvres qui réfiftent aux fleurs de larnica, Collin ne nie pas que le quinquina ne leur foit préférable, lorfqu'il faut arrêter ces ficvres menaçantes qui enlèvent les malades au troifième ou quatrième accès. L'arnica réuffit, fur-tout, lorfquon doit atraquer des humeurs âcres \& vifqueufes, \& qu'il faut détruire le coma vigil, la phrénélie, le météorifme, les ergorgemens des vîfcères, \&xc,

(I) Ratio meçendi, to I, pag. 86.

(3) Ibid. pag. 88, 
occasionnoit des douleurs connues sonis le nom de rhumatisme goutteux ( $\mathrm{I}$ ).

Les fièvres ardentes bilieuses sont l'opposé de celles dont nous venons de parler. Aussi la manière de les traiter est-elle différente. Dans ces fièvres, qui sévissent pendant des constitutions excessivement sèches et chaudes, les bains sont d'un grand secours; et M. Polippé Despories s'en servit avec avanage dans l'épidémie rémittente double-tierce qui régna a St. Domingue pendant la constitution de 174.2 , remarquable par sa sécheresse (2). Si ces fèvres prennent la tournure de celles dont il a étéfát nontion ( $\$$. XCII. $\mathrm{n}^{\circ} \cdot 2$ ), comme cela arrive sonvent, on ne sauroit les combattre avec une méthode plus réléchie et mieux ordonnée, que celle qu'on leur a assignée; et lorsqu'elles occasionnent une dégénération bilieuse considérable, ce qui leur est encore aussi commun, on ne pourroit trop tôt les arrêter avec le fébrifuge qui, dans ce cas, est un grand antibilieux, suivant les expressions de Binchi (3), et remédie sipérieurement, selon de Harn (4), alix vices du systeme bilicux.
(1) Ilid. pag. 89.
(2) Hifroire des maladies de Saint-Dominguc.
(3) Fiffor. hepat. t. I , p. 25 I (prafidiun antibiliare.)
(4) Ratio medendi, t. VI. p. 2 s. 


\section{De l'usage du Quinquina}

(*) Madame V..... l'épouse de celui dont il a été parlé dans le $\$$. LIX, s'alita deux jours après la mort de son mari, ainsi que son fils unique, garçon de 20 ans, robuste et bien constitué. Des idées de contagion vinrent alarmer les malades et leurs proches, et nous eumes bealicolip de peine à rassurer leurs esprits inquiets.

Madame V.... fut émétisée avec l'ipécacuanha après le premier paroxisme; elle fut purgée après le second qui se renouvela en tierce. Le quatrième jour de la maladie, qui devoit être un jour libre, il y eut un redoublement analogue à celui de la veille: on l'attribua aux fatigues de la purgation. Le cinquième jour donna une reprise assez forte, et le teint de la malade devint jaune; les urines étoient foncées; la pean du corps ni le blanc des yeux ne prirent point cette couleur. On réitéra le purgatif le sixième jour, et il survint une exacerbation semblable à celle du quatrième jour : les selles furent assez copieuses, aisées; la matière étoit bilieuse et fécide, d'une consistance moyenne. Le redoublement di septième jour, sans être plus violent en apparence que celui du cinquième, fut marqué par de plus grandes auxiétés; il'y eut des vomissemens d'une matière amère, des selles spontanées d'une mauvaise odeur, et la couleur 
jaune du visage devenoit plus intense. La malade demandoit le quinquina. On le lui donna à la dose d'une once en substance divisée en quatre prises égales. Dans le cours da huitième jour, il y eut un paroxisme moins fort que le précédent, quoiqu'accompagné des mêmes symptômes : la langue qui avoit été chargée se dépouilla. On répéta le quinquina de la même manière, et le redoublement du neuvième jour ne laissa pas de venir : les épiphénomènes des reprises précédentes étoient moindres. Le dixième jour on suspendit les remèdes; il n'y eut aucune exacerbation, et la malade se trouvoit mieux. On revint, le onzième jour, au quinquina ì la dose de demi once, et le mieux fut plus sensible; le visage fut entièrement dépouillé ainsi que les urines; tous les symptômes qui indi. quoient l'érétisme des premières voies, étoient calnés. Le quinguina fut continué pendant les deux jours suivans à la dose de deux drachmes, et la convalescence fut décidée.

Cetce observation est une preuve de l'eficacité du quinquina pour arrêter la dégénération bilieuse des humeurs, et les épiphénomènes qui dépendent de la matière bilieuse âcre et abondante. Il n'a pas été question de M. V.... le fils. Sa maladie ne présenta aucune circonstance particulière, et n'a pas mérité d'être décrite. 


\section{De l'usage du Quinqnina}

XCV. Tels sont ( $\$$. XCI. à XCIV.) les préceptes cliniques relatifs aux indications du quinquina considéréss du côté du génie ( $\$$. XXXI à XXXIII ) des fières rémittentes. S'il étoit possible de s'expliquer nettement sur les nuances qui, mettant des différences réelles, quoique insensibles, dans le caractère des maladies, doivent faire varier ladministration du quinquina, dans combien de détails ne faudroitil pas entrer encore? Le point principal pour le meilleur emploi de ce médicament, n'est pas uniquement de saisir quel est le génie dominant pendant le cours d'une fièvre, ou dans ses divers périodes; il consiste encore à ne pas se méprendre sur les modifications que les épidémies annuelles reçoivent de la diversité des saisons, et même d'un changement accidentel dars la température. On a vu, par exemple, que des fièvres bilieuses qui n'admettoient pas le quinquina, pendant une saison sèche, éroient traitées avec beaucoup de succès au moyen de ce fébrifuge, aussi-tôt que des pluies salutaires avoient rafraichi l'atmosphère et répandu une douce humidité ( $\mathrm{I}$ ) : on a vu, dans le cours d'une longue épidémie, que le génie inflammatoire avoit dominé pendant toute une année,

(I) Voy. Journal de méd, t. LV.p. I8I. 
et conséquemment que l'usage du quinquina avoit dî être précédé par les saignées et les anti-phlogistiques, tandis que l'année d'après le génie avoit été putride et malin, contrindiquant les évacuations sanguines, et réclamant le quinquina dès le principe ( $\mathrm{I}$ ) : en un mot, on a vu qu'une fièvre rémittente, qui, sans perdre son caractère primitif, prolongeoit sa durée pendant une ou plusieurs années, prenoit sur la fin de l'hiver une nuance inflammatoire; au printemps une nature catarrheuse; au commencement de l'été un fond putride; en automne un génie bilieux; et enfin, une tournure attrabilaire à l'époque propre à cette dégénération particulière : en conséquence, que l'administration du quinquina devoit être tantôt précoce, tantốt retardée, tantôt indispensable, tantôt dangereuse, et que les secours préliminaires ou concomitans devoient être, dans les divers temps, d'une nature diamétralement opposée. Un médecin instruit, et qui, judicienx observateur, sait lier tous les rapports, n'est point trompé par ces métamorphoses; il réfléchit sur linfl::ence des saisons, sur celle des variations atmosphériques; il écoute la nature, pour savoir quelles sont les crises qui jugent la mala-

(I) Voy. l'épidémie de Lafchendorf dans Lautter, pag. $24-8,4 I$. 
254 De l'usage du Quinquina

die régnante, même quelles sont celles qui terminent les maladies contemporaines : riche de ces connoissances, il place avec discernement les remedes majeurs, et leurs succès sont proportionnés à l'à-propos de leur administration. L'observation des effets des moyens suratifs supplée, dans certains câs, à l’obscurité des indications; car il est des maladies qui semblent exactement de la même nature, et dont l'action des médicamens annorce seule la diversité. Dans l'épidémie qui affligcoit, en 1773 , les habitans d'Escala et de Molère, distans d'en. viron une lieue l'un de l'autre, tous les signes arnonçoient dans les deux endroits une vraie fièvre ptitride des secondes voies. Cependant le camphre et le quinquina produisoient de très-bons effets à Molère, et nuisoient beaucoup à Escala. C'étoit tout le contraire à l'égard de la crême de tartre, des tamarins et du petit lait, les malades d'Escala les supportoient à merveille, et en étoient très-soulagés, et ceux de Molère sentoient leurs maux s'aggraver par leur usage (I).

XCVI. Les constitutions é:ant supposées légitimes, et l'ordre des saisons qui leur est subordonné, étant légitime aussi, les fièvres simples du printemps ont un génie inflammatoire ( $\$$.

(I) Journal de Médecine, t. XLV, pag. I44. 
dans les fièvres rẻmittentes.

XXXVI ), une marche graduellement aiguë, et beaucoup de tendance à une prompte crise. Elles n'admettent donc point l'usage du quinquina : en vain, pour l'ordonner, s'étayeroiton de la dégénération de la fièvre, de ce caractère en apparence corrupteur, comme le dit Torti, qui', lui ayant fait perdre son premier type, peut multiplier et accroitre l'intensité des accidens. Comme les indications de ces accidens ne sont pas subordonnées à celles du levain fébrile, on ne les rempliroit pas avec fruit au moyen du quinquina. On doit lui préférer les secours appropriés ( $\$$ X.CII) au génie de la fièvre qu'il faut directement combattre; et si l'irrégularité putride succède ensuite et prend la place du génie de linflammation, alors, indiqué par le type rémittent, le quinquina sera bien placé, ses succès ne seront pas équivoques.

Ce n'est pas toutes fois que le quinquina ne puisse être employé dans les fièvres rémittentes du printemps, avec succès et sans danger. Nous disons seulement que cette écorce est contr'indiquée par le génie légitime des fièvres de cette saison, et qu'elle ne peut être ordonnée que pour satisfaire à d'autres indications.

XCVII. Il en est tout autrement des fièvres du génie putride ( $\$$. XXXI) qui , d'abord intermittentes, passent très-vite au type rémittent, 


\section{$25^{6} \quad$ De l'usage du Quinquina}

ou de celles qui, rémittentes dans le principe; acquierent dans peu le type continu. Dans les premières, le quinquina doit être placé aux. moindres signes de dégénération ultérieure; dans les secondes, ce fébrifige est indiqué maigré lextrême obscurité de la rémission, et l'on n'a rien à redonter de son usage. Nous disons pius, i] n'y a que le quinquina qui puisse les guérir, ainsi que Muzell (I), Senac (2) et autres l'ont décidé par des exemples. Mais avant d'administrer ce spécifique, il est de la prudence de tenter de procurer une rémission: on y parvient, suivant les circonstances, en appliquant à propos une saignée, l'émétique, un purgatif, des mixtures salines, ou le bain tempéré, comme le veut Sarcone (3).

XCVIII. Les fièvres automnales, d'abord très-aiguës, ont une marche graduellement chronique ( $\$$ XXXVII ); leur génie est foncièrement putride ou bilieux, et leurs indications manifestes scnt de recourir au quinquina, dès que la cause humorale a été long-temps combattue, à moins que des épiphénomènes tffrayans et dangereux n'obligent à administrer de bonne

(1) Medicinitche nud Chirurgifche Wahmehnungen, \&c. , Ire./ collect.

(2) De recondita febrizm, \&c. , pag. 4 I8.

(3) Hiftoria ragionata de mali, \&xc., t. I, p. I99.

heure 
dans les fièvres rémittentes:

heure ce fébrifuge. Ici, comme dans le cas précédent, le quinquina devient indiqué par la dégénération de la fièvre en continue, pourvu que, par un retard inconsidéré, on ne donne pas le temps aux produits morbifiques de dominer sur les indications de la fièvre, et de constituer ainsi des contr'indications pour l'emploi du fébrifuge.

$\mathrm{XCIX}$. Ces considérations nous guidèrene pour l'administration du quinquina dans le traitement de l'épidémie de 1780 , dont nous avons déjà fait mention ( $\$$.XLI); épidémie qui, par linfluence de la constitution, participoit du caractère des fièvres du printemps et de l'automne. Tant que l'anomalie ou l'intensité des symptômes n'inspira pas de justes craintes, nous abandonnâmes la fièvre à elle-même, suivant avec attention les indices d'orgasme, de pléthore ou de raréfaction dı sang, pour placer Ia saignée; les signes de turgescence on de ftagnation des saburres, pour administrer les purgatifs que nous répétâmes, tantôt pendant tout le cours de la fièvre, tantôt seulement dans son commencement et son déclin, respectant son état pour ne pas déranger les mouvemens critiques (I). En se prêtant ainsi aux vues de la na-

(1) V. Richter de cocibonum presfdiis, evancuantiun, $a b u f$ is ever/ss, dans le a volume de fes $O$ pufcula medica . p. 307. 


\section{De l'usage du Quinquina}

ture, nous parvinmes quelquefois à la fin de is maladie, sans avoir vu naitre les occasions de donner le quinquina. Les foyers humoraux étant détruits, le levain fébrile étoit sans force, peutêtre faute d'une matrice propre à son explosion, ou étoit entrainé vers quelque émonctoire par l'effet de cette puissance salutaire qui opère la dépuration et les crises. Mais, dès que le génie féroce de l'épidémie s'exprimoit par la subintrance des exacerbations, lorsque nous nous apercevions d'une dégénération prochaine par la diminution du froid, la rareté et l'obscurité des frissons, et la briéveté de ce premier temps du paroxisme, par l'augmentation de la chaleur, sa grande intensité et l'extension de sa durée, enfin par la privation ou le peu des sueurs ou moiteurs par la rougeur des urines et l'absence du sédiment, par l'inquiétude des malades et par l'obscurité et la briéveté de la rémission: quand les exacerbations présentoient dans leurs premiers périodes, un froid glaçant, des anxiétés insupportables, des défaillances, des toux déchirantes, etc.; dans leurs seconds périodes, des vomissemens soutenus, des fortes angoisses, un météorisme douloureux, un délire avec un pouls disproportionné, un assoupissement, des affections paralytiques, une difficulté d'uriner, etc; dans leurs troisièmes périodes, de la soif, des 
inquiétudes, un affaissement singulier, un pouls déprimé s'il avoit été développé ou non dans l'exacerbation: pour lors le quinquina devin notre unique espoir ; nous le donnâmes à pleines mains et de toutes les manières : on auroit dit que nous en abusions, si le cas eût été moins pressant, si la fièvre eût été moins grave, si les succès eussent été moins multipliés et moins complets.

Les complications furent néanmoins le motif de plusieurs variétés dans l'administration du fébrifuge. Tantôt les circonstances faisoient accélérer l'application de ce secours's, tantôt elles en retardcient l'usage, tantôt enfin, elles n'en permettoient jamais l'emploi. Mais n'oublions pas de le remarquer: ce sont les circonstances opposées à l'application du quinquina qui rendoient ce médicament nuisible; et ce sont des circonstances semblables qui sans doute ont fait plus d'un détracteur de ce remède précieux. Puissions-nous détruire leurs argumens en nous expliquant sur ces complications; mais générali-i sons nos vues et réduisons nos observations enj préceptes.

C. Dans l'opinion de M. Médicris ( 1 ); le quinquina n'agit que par sa vertu tonique, et propre

(I) Gefchichte periodicher kranckeictern, \& $\mathrm{c}_{\text {c }}$

$$
R \text { ) }
$$




\section{De l'usage du Quinquina}

à diminuer la trop grande irritabilité des intestins et du ventricule. Mille exemples annonçent, en effet, que ce fébrifuge possède réellement la vertu de réduire, ̀̀ un état moyen, ce vice fâcheux dit système des forces motrices ( $\$$. XLIII ); et sous ce point de vue, il n'est point de sujets à qui ce remède convienne plus spécialement qu'aux femmes grosses (I), parce que la grossesse décide toujours une augmentation d'irritabilité et de sensibilité, qui, le plus souvent, tombe et se dissipe après elle. Dans cét état, si la fièvre n'est pas des plus simples, on doit craindre $I^{\circ}$. que lirritation permanente de l'uterus, ne rende cet organe le foyer des métastases, le noyai de lirritation; $2^{\circ}$. que la pléthore qui, pour lordinaire, accompagne la grossesse, ne sollicite des pertes utérines qui décident l'avortement; $3^{\circ}$. que la stase des sucs dans les viscères du bas ventre, refoulés par le développement del'uterus, ne fournisse au transport d'humeurs sur la matrice, ou ne favorise une inflammation des entrailles. Mais plus le quinquina devient indiqué par l'urgence du cas, plus il faut se hâter d'en assurer les effets. Chez les femmes grosses, les saignées en sont le moyen le plus assuré,

(1) Voy. Darluc, journal de Méd. t. XVI, p. 35I, jos; Laitter, Hift. bien. morb. rur. p. I8, \&c. 


\section{dans les fièvres rémittentes.}

comme le plus indispensable. Guidetti ( 1 ) a vu le défaut de ce secours préliminaire, produire une vraie perte utérine ct même la fausse couche. Nous avons vu nous-même le quinquina donné à une demoiselle de 14 ans et quelques mois, qui, pubère encore, présentoit tous les indices du prochain établissement des règles, décider un spasme si violent dans l'hypogastre, "qiion crut que l'inflammation ne tarderoit pas à survenir. On saigna la jeune malade trois fois en I 8 heures, on fomenta la région hypogastrique, on réitéra les demi-lavemens émolliens, on donna le camphre avec le nitre; et comme les exacerbations de la fièvre augmentoient en intensité, on revint, le surlendemain des saignées, au quinquina à forte dose, mais uni avec les opiatiques, et notre malade ne tarda pas à entrer en convalescence. Il y a lieu de croire que le quinquina n'eût produit aucun fâcheux accident, si les saignées avoient été pratiquées. L'âge de puberté offre, comme on le sait, autant de ménagement à garder que l'état de grossesse.

Quelque précieuse que soit la combinaison du quinquina et des opiatiques (2) pour assurer

(1) Dans Bianchi hiftor. hepat. t. I, p. 636, f. 20, p. 637, g. 21 .

(2) Plutieurs Auteurs ont vanté l'eficacité dé l'opiun pour la cure des fièvres intermittentes. Hippocrate $8 x$

$\mathrm{R}_{3}$ 
les succès du fébrifuge dans les cas d'un vice d'irritabilité, on se flatteroit en vain d'admi-

Galien recommandent la graine de jufquiame, qui eft une efpèce de narcotique. Les Difciples de Paracelfe parlent expreffément de l'opium. Rondelet propofe la thériaque dans le vin. Rivière of domer l'opium pour prévenir l'accès d'une fièvre rémittente, maligne, foporeufe. Enfin, M. Berryat reffufcita une méthode oubliée. Lind (an elfai on difeafes, \&ac.) prodigue les plus grands éloges à l'opiun donné pendant la chaleur de I'accès, \& outre les vertus fédatives quill lui attribue, il dit que c'ęt le meilleur remède qui puiffe être employé pour préparer les malades à l'ufäge du quinquina, \& gu'il ne réuffit jamais mieux, que lorfqu'on attend, pour le donner, que la chaleur du paroxifme commence. M. Olivier nous apprend (Journal de Méd. t. XI, p. 33 I) qu'il a été fort heureux en plaçant 3 ou 4 heures avant laccès la potion fuivante, faite avec la décolion de deux gras de quinquina, d'une pincée de rofes rouges, y ajoutant un grain de camphre, \& Is à 20 gouttes anodines. M. Duchanoy a publié, en I 780 , un Mémoire fur l'ufage des narcotiques dans les fièves inter. mitcentes; \& quelques Médecius de Paris (Extrait du prima menfis, Journal de Med. t. LVI, p. 84.) ont confirné leurs bons effets. Enfin, M. Schartlich a donné dans fa differtation (de ufu opii in febribus intermittensíbus ), des détrails intéreflans fur cetie inatière. Pour finir a nous parlerons des fuccès que le Docteur Monro eut dans le kraitement des fièvres bilieifes rémittentes de l'hôpital de la Martinique en I 76 I, en domant le quint quina uni à l'opium, à caufe de la grande irritabilité de l'eftomac. Mais l'opium ne faifoit rien à perice dere, 8. faifoit mal avant d'avoir nettoyé le canal inteftinalo 
dars les fièvres rémittertes.

nistrer avec fruit ce spécifique, lorsque le vice d'irritabilité est monté jusqu'à un certain point, et parvenu, pour ainsi dire, jusqu'à l'excès. Un remède tout-à-la-fois fébrifuge, astringent et tonique, opère alors, ou donne le résultat d'un âcre et d'un stimulant. Combien de fois ne s'en est-on pas convaincu, en voulant adapter le quinquina aux cas d'affection nerveuse qui demandent véritablement la méthode aqueuse de M. Pomme? Seroit-ce parce qu'un systènie trop irritable qui ressent vivement une inpression astringente, sans pouvoir en recueillir le fruit, puisque toute action brusque et forte dérange plutôt l'ordre des mouvemens qu'elle ne le rend régulier, doit en être plus violemment ébranlé, et que l'excitation subite qui en provient, doit pareillement amener plutôt le spasme qu'un ordre tranquille et naturel?

Quoi qu'il en soit, nous sommes persuadés qu'il est en général très-difficile de faire prendre le quinquina, comme fébrifuge, à des femmes douées de beaucoup de sensibilité et excessivement irritables. Nous Iui avons vu produire alors des souffrances spasmodiques, cruelles, la suppression de toutes les excrétions, l'augmentation de tous les accidens fébriles. Cependant le quinquina avoit été combiné, soit avec des

$\mathrm{R}_{4}$ 


\section{De l'usage du Quinquina}

narcotiques, soit avec de simples sédatifs, soi enfin avec les meilleurs anti-spasmodiques.

(*) Madame Fournier, apothicaire, tombe malade; sa fièvre est celle de la constitution, et son tempérament est très-irritable et trèssensible; de plus elle est nourrice. Les redoublemens marqués en double tierce, commencent à être orageux au troisième paroxisme des jours impairs. Un émétique et deux purgatifs avoient déjà nettoyé les premières voies. Je conseillai le quinquina à la dose de six gros, mêlé avec un peu de magnésie et quelques grains de sel sédatif, pour quatre doses à prendre dans l'intervalle de deux exacerbations. La première prise du fébrifuge excite des accidens affreux; tels que des envies de vomir très-fatigantes, des crampes très-douloureuses dans l'estomac et dans tous les membres, des menaces de syncopes très-rapprochées. On suspend le quinquina; on administre la tisanne de poulet, acidulée avec la liqueur minérale anodine d'Hoffmann; on place sur l'épigastre et à la plante des pieds, un emplâtre de galbanum avec camphre et opium; on dome un lavement avec linfusion des feuilles darmoise et les fleurs de camomille, Le calme renait ế le paroxisme de ce jour est emporté. Celui du lendemain ne revient pas non pius, et la malade entre en convalescence. 
Pour exciter lappétit très-languissant, on usa d'une teinture aqueuse faite avec le quinquina, la rhubarbe, les fleurs de caillelait et de illeul: ce qui réussit à merveille.

Dans cet exemple, on voit le quinquina produre de facheux effets dans une constitution très-iritable; mais n'y voit-on pas aussi les preuves de la vertu fébrifuge de cette écorce, puisqu'une aussi petite quantité put arrêter les paroxismes d'une fièvre rémittente. Il est bon peut-ôtre de faire observer que le quinquina qui fut employé, est le quinquina rouge, dont nous parlerons bientôt.

CI. Une grande atonie n'est guères plus farorable à l'emploi du quinquina, qu'une excessive irritabilité; et cette proposition pourroit bien n’être regardée que comme un paradoxe, si l'expéíience n'obligeoit d'en décider autrement. Soit qu'avec beaucoup d'atonie ( $\$$ XLIV.); le corps surabonde en humeurs cries, inertes, qu'il est dangereux de fixer; soit que l'économie animale ne puisse passer subitement sans péril, d'un extrêne à lautre; soit que, dans limpossibilité que tout le corps puisse être également fortifié, le ton de quelque partie ne se relève qu'aux dépens de quelques autres; soit enfin, que, faute d'une répétition sympathique de l'excitation que le remede fortifiant procure à 


\section{De l'usage du Quinquina}

lorgane sur lequel il s'applique, l'action du remède, concentrée sur un organe, n'occasionne un spasme partiel, d'autant plus dangereux, que toutes les antres parties sont dans un relâchement morbifique : il n'en est pas moins vrai que le quinquina (I) nuit le plus souvent, lorsque l'atonie est extrême, et qu'il procure alors, sans pouvoir presque l'éviter, des obstructions, la jaunisse, l'hydropisie, etc., comme on peut le croire d'après les facheux effets que l'emploi du quinquina produit assez souvent en Hollande (2), et que nous avons observés sur des pêcheurs, auxquels on avoit mal-d̀-propos et sans ménagement administré le fébrifuge. Dans ces constitutions, suivant lidée de Celse, la fièvre contribue autant à la guérison, que le feu contribue à l'amélioration dos terreins humides. Aussi est-on plus heureux avec l'usage du musc, du castoreum, du camphre (3), de l'arnica, des

(I) Voy. les penfẻes fur le relâchement du corps hul *dain \& fur l'abus du quinquina dans ee cas \& autres (en Anglais, r783.)

(2) Voy. l'hifoire géographique, phyfique, naturel!c \& civile de la Hollande, par M. Lefranq-de-Berkey, Doat. en Méd.

(3) Voy. fur les vertus de ce remède, les Auteurs cités à la päg II 6 , note $a$, \& Gejner, découvertes des temps les plus modernes en Médecine (en allem.) 
frictions sur la peau pendant lintervalle des piroxismes; et quand on a recours au spécifique, il faut adopter ses teintures spiritueuses, et notamment des préparations analogues à la mixture fébrifuge purgative suivante. Prenez trois onces de décoction faite avec une drachme et demie de quinquina concassé, six gros de sel cathartique amer et deux gros de teinture simple de quinquina pour une dose (I). Dans les cas oil on a besoin d'évacuer et d'arrêter promptement la fièvre, ce médicament est un purgatif et un fébrifuge très-actifi ; il est principalement destiné au traitement des fièvres rémittentes et intermittentes des contrées humides.

Mais l'atonie n'étant pas portée à l'extrême; suffisant seulement pour rendre les mouvemens de la nature imparfaits et trop foibles, le quinquina ne sauroit être différé sans danger. Un délai mal entendu favorise la formation des stases ou des engorgemens, toutes les excrétions languissent ou sont suspendues. Le levain fébrile qui ne s'évacue pas, reste dans le sang, pour l'altérer, le décomposer; son évacuation, lorsqu'elle a lieu, est presque l'effet du hasard,

comparez Wagler de morbo mucofo; p. 89, Darlue, Journ. de Mélec. t. XVI, p. $362,8 \times c .$, \&c.

(I) Voy: Lewris, nowveau difpenf. t. III, p. is 
ou dépend des efforts extraordinaires de la nature qui n'a pas toujours de telles ressources; enfin son dépôt sur quelque viscère essentiel, qui est l'événement le plus commun, entraine des obstructions, la cachexie, l'hydropisie et tous les maux qui marchent à leur suite. Les gens mal-aisés sont sur-tout menacés de ces accidens, lesquels donnent lieu à une maladie toujours longue et conséquemment ruineuse. Chez eux, on a à combattre une vraie inanition, produite par le besoin ou par la mallvaise nourriture (I); la nature est souvent peu capable de lutter contre le mal, parce qu'elle est épuisée par un travail pénible et prématuré. Le quinquina renforcé même par l'action du camphre, leur convient donc à tous égards; il leur tient lieu d'analeptique, de fortifiant, de fébrifuge. Nous avons alors vu quelques doses de quinquina en substance ou en décoction dans du bon vin rouge ou blanc ( $\$$ LXXXII), selon le besoin, arrêter presque subitement la fièvre. Nous avons vu son action rétablir le

(1) Nous croyons devoir remarquer ici dans un zemps où plufieurs Médecins font la médecine parmode, qu'il faut bien fe garder de prefrire à ces malacies une diète végétale pour combattre des accidens putrides: on redoubleroir leurs maux, on donneroit des ailes à la putridité. Voy. Quarin de febrib. pag. 42. 
dans les fièvres rémittentes.

ton des solides, ranimer les crises, et changer, comme par enchantement, les évacuations fétides et séreuses, en matière d'une meilleure qualité. Le quinquina ne feroit-il que rétablir ou augmenter la transpiration intestinale, qui doit être bien grande, si l'on fait attention à la grande surface interne du conduit intestinal ( elle est de 15 pieds ou égale à la surface de la peau), qu'il produiroit encore les meilleurs effets.

CII. Le quinquina n'est ni moins nécessaire ni moins essentiel, lorsque, dans une constitution gênéralement forte et saine, il se trouve un organe débile et disposé par-là à de grandes lésions; ce qui se connoît, entr'autres signes, parce que cet organe est si affecté du paroxisme, qu'à raison de ce, le malade est, à chaque reprise, dans un danger de plus en plus imminent. On diroit que, dans ces circonstances, le mal ne se fait sentir que sur l'organe affoibli. Nous avons vu des sujets énervés par des purgatifs répétés, avoir, dans chaque exacerbation, des météorismes affreux avec douleur, que le quinquina dissipoit avant que la fièvre fût totalement guérie. Grant parle de violens maux de tête (I) dans les uns; dans les autres, cesont des en-

(I) Recherches fur les fièvres, t. I, p. 108. Il eft effentiel de conférer ce paffage pour bien placer lo fébrifuge. 


\section{De Lusage du Quirquina}

gorgemens et étranglemens, de cruels vomissemens

(I), douleurs, crampes considérables, gonflemens de l'abdomen (2), qui, n'arrivant, pendant le paroxisme, que par une suite d'une foiblesse particulière aux parties lésées, demandoient que, sans balancer, on administrât le fébrifuge pour arrêter la fièvre et ses fâcheuses conséquences. On peut voir ailleurs ( $\$$. LXIX) ce que nous avons dit sur les dangers de différer l'emploi du quinquina dans les cas où l'on pouvoit craindre que l'affection subordonnée ne devint dominante.

${ }^{*}$.) Mademoiselle Chassanis, fille de 45 ans, me fait appeler pour une cardialgie violente, avec des angoisses et une gêne affez forte dans les mouvemens de la respiration. Elle avoit eu déjà deux attaques pateilles moins violentes, et dans leurs intervalles, la malade s'étoit ressentie du mal-aise, de l'agitation; sa bouche étoit mauvaise, sa tête étoit un peu pesante, et il y avoit par tout le corps un sentiment de lassitude. Jordonnai une mixture anti-spasmodique et calmante. Le lendemain matin, les accidens étoient calmés. L'épigastre étoit encore très-sensible à la pression; et, à la cardialgie près, la malade

(I) Ibid. P. III.

(2) Ibid. p. II 
étoit dans l'état dont j’ai déjà fait mention. La nuit suivante fut bonne; le lendemain matin, je donnai un purgatif qui réussit très-bien. La cardialgie revint la nuit d'après avec la même inteasité; ce qui fut attribué assez légèrement à laction du purgatif de la veille. Les souffrances furent si vives que la malade crut ne pouvoir pas y résister. Les anti-spasmodiques, réunis aux calmans, produisirent encore de bons effets. Cependant le retour périodique de cette car-: dialgie me porta à prendre d'exactes informations sur les deux attaques que je n'avois pas observées; et je me convainquis par l'ordre alternatif et réglé des reprises, et les symptômes qui avoient lieu dans les intervalles, que la malade éprouvoit la fièvre épidémique de la constitution, mais déguisée sous les dehors de la cardialgie. J'appris que la malade avoit autrefois beaucoup souffert de l'estomac, et que ce viscère étoit, chez elle, habituellement foible et irritable. J'ordonnai le quinquina à forte doses. On m’opposa quelques tésistances, et ce remède fut différé. On tint le ventre libre avec des lavemens; on appliqua fur l'épigastre, un écusson antispasmodique et fortifiant (I). Après 24 heures

(I) Prenez une ou deux onces de diabotanum; quinze ou vingt grains de camphre; huit ou douze grains d'opium srad; une ou desur dochmes de feuilles do 


\section{2}

\section{De l'usage du Quinquina}

de calme, la cardialgie reparut avec les accidens qu'elle avoit coutume de produire; et la malade convaincue prit le quinquina, à la dose d'une once, qui emporta sans retour les réprises de cette fièvre rémittente cardialgique. L'état des premières voies exigea deux purgatifs dans la convalescence; ils furent suivis du quinquina. A la fin du second septénaire, il survint des accès de fièvre intermittente qui furent guéris après l'émétique et deux purgatifs, par le fébrifuge et les eaux de Balaruc.

Il faut bien distinguer néanmoins, entre la foiblesse particulière d'un organe qui le rend susceptible de quelques lésions, et cette foiblesse particulière d'un organe qui l'a rendu le foyer de quelque congestion humorale, ou le centre d'un spasme fixe pendant lincubation de la maladie. Dans ce dernier cas, le quinquina ne doit être placé qu'après la résolution de l'etı gorgement ou du spasme; il n'y a qu'un péril prochain, qui puisse autoriser ì procurer un répit, en plaçant cette écorce à bonne heure. C'est ainsi que dans la cacochylie mésentérique qui complique les maladies de certains sujets alonnés à la bonne chère, le quinquina ne macis en poudre, \& fuffifante quantité de baume diz Péroul, pour faire, felon l'art, un écufon de grandeur convenable pour appliquer fur l'épigaftre. 
peut étre employé qu'avec la dernière précalltion et la sage retenue de Baglivi.

CIII. Si l'atonie et l'irritabilité indiquent et contrindiquent également le quinquina dans les fièvres rémittentes compliquées de ce vice, on sent qu'il convient de réfléchir bien mûrement, lorsqu'il faut administrer ce médicament dans l'enfance ou dans la vieillesse. Les enfans sont ordinairement dans le cas du vice d'irritabilité; mais entremêlé de foiblesse; et les personnes âgées sont communément dans celui de l'atonie, mais compliquée de rigidité dans les solides et d'épaississement dans les sucs. Aussi doit-on suivre d'un œil vigilant les effets de la fièvre; dans ces deux termes opposés de la vie humaine: La constitution se déprave aisément dans l'enfance; la vie s'éteint facilement dans l'âge avancé. De-là, les indications urgentes de placer le quinquina, dès qu'on le peut sans danger; contre un mal qui peut occasionner d'aussi cruels ravages.

CIV. Dès qu'on connoît les effets naturels du quinquina et qu'on est instruit des accidens qui menacent les personnes dont les fibres pêchent habituellement par trop de délicatesse ou par trop de rigidité ( $\$$. XLVII ), il est bien facile de s'apercevoir que la fébrifuge est généralement mauvais dans l'une et l'autre circonstance. 


\section{4}

\section{De l'usage du Quinquina}

Des vaisseaux trop délicats se rompent aisément lorsqu'une vive astriction resserre leurs calibres, sans compter que les effets mêrne de la fièvre dans les tempéramens dont tout le système pèche par délicatesse, ne sont pas de nature à être prévenus par le quinquina, puisqu'ils dépendent d'un vice de constitution antérieur à la fièvre. En effet, le quinguina ne réussit jamais bien que lorsque, dans une constitution délicate, les humeurs sont délayées et acrimonieuses, les solides flasques et mous. Mais la roideur des fibres est beaucoup plus opposée encore à l'administration de ce médicament; car, dès qu'il possède une vertu tonique, astringente, qu'il remédie à la dissolution des fluides, en conserve la crase, en rétablit la consistance (I), il ne peut être que contr'indiqué par un tempérament athlétique où des fibres fortes et roides réagissent sur des liquides grossiers et presque glutineux. Il ne peut être aussi que contr'indiqué par le plus haut période de l'âge viril, pendant lequel l'énerơie des vaisseaux pousse avec beaucoup d'activité, un sang riche,

(I) On a long-temps cru dans les écoles, que le quinquina a une action diffolvante fur le fang, \& Stork paroît même n'être pas fort éloigné de cette opinion. Quant à nous, il nous fenble qu'on peut prouver que, fuivant les cas, le quinquina épaifit ou difout les fuides. 
des humeurs presque trop consistantes; il ne peut être enfin que contraire dans tous les cas oì la fibre, trop sèche, trop tendue, se trouve avec des fluides dont la partie séreuse n'est pas en proportion de la partie concrescible, comme cela se rencontre assez généralement chez les sujets que le métier expose à un feu violent et soutenu. Il est à remarquer, par exemple, que les maréchaux, les serruriers, principalement sujets à des fièvres inflammatoires, dont le siège est au ventre, et d'un tempérament trèssec et très-aricle, ne prennent pas le quinquina sans être exposés aux fièvres ardentes, aux fièvres lentes, etc. Ramazzini a déclaré ce fébrifuge très-préjudiciable aux chasseurs (I) et $\mathbf{M}$. Darluc nous dit que le quinquina administré, sans trop de précaution, à deux sujets robustes et nerveux avant la reprise, leur fit perdre toute connoissance. Nous les trouvâmes, ajoute-t-il (2); dans le chand de la fièvre, avec un pouls irrégulier, vif, tremblottant, agités de mouvemens convulsifs dans la partie supérieure de l'abdomen, qui partoient visiblement du diaphragme; ce fâcheux état éluda tout secours (3).

(I) Malad. des artifans, trad. franç. p. 565 .

(2) Journal de Médecine, t. XVI, pag. 37 I, 2.

(3) Au lieu de recourir au quinquina dans les cas qui font'objet du S. CIV, on ne peut employer riea 


\section{${ }_{276}$ De luusage du Quinquina}

CV. Quand l'humeur goutteuse ( $\$$. XLVIII) est mise en mouvement par la cause matérielle de la fièvre, elle présente, pour l'orclinaire, des indications plus urgentes que le levain fébrile et des contr'indications pour l'usage du quinquina, du moins pour son usage précoce. L'objet majeur est de prévenir la fixation de la matière arthritique, et d'en favoriser le dépôt sur les jointures. Or, si la goutte est inflammatoire, on ne peut lui opposer rien de mieux que les moyens nécessités par la fièvre; on a seulement attention de faire au pied les saignées zequises, de multiplier les pédiluves, et d'insister sur les boissons tempérantes. Au contraire, si la goutte est froide, on se relâche sur les secours indiqués par la fièvre, pour user des doux diaphorétiques et des pédiluves âcres. Les purgatifs sont doublement utiles, et il faut d'autant moins en différer l'administration, que les saburres stagnantes, par leur irritation topique, peuvent appeler l'humeur goutteuse. Mais après

de mieux approprié que les bains tiedes; moyen qui cependant eft auffi utile que négligé. Voyez les réflexions intéreflantes qu'on trouve dans le $63^{\mathrm{e}}$. vol. du Journ. de Méd. pag. 183. On y voit que les idées de putridité ne doivent point éloigner de l'emploi des bains, ni même l'époque avancée de la maladie, $8 x$ la foibleffe apparente du malade. 
dans les fievres rémittentes.

leur opération, il est bon d'ordonner un doux stomachique; et la thériaque remplit assez bien cette vue, comme celle de pousser au dehors. On sait que la liberté du ventre dispense souvent de rappeler la goutte aux extrêmités, et si cette indication est urgente, on prescrit ou des pédiluves animés par la moutarde, ou des sinapismes irritans, ou des embrocations avec la teinture de cantharides, ou bien, enfin, les vésicatoires (I). C'est lorsqu'on est rassuré sur la matière arthritique, qu'on pense à remplir l'indication de la fièvre par le quinquina. Pour l'ordinaire, il ne réussit parfaitement que sur la fin de la maladic, où le quinquina devient indiqué par la goutte et par la fièvre. Pcut-être faudroit-il préférer le bois de Quassie (2) à

(I) Voy. M. Stevenfon, a fucce/sful wéthod. of treating the gout bybliftering, \&xc. \& M. de Berger, fur l'efficacité des bains de moutarde dans les douleurs goutteufes \& arthritiques.

(2) Depuis qu'un Suédois (M. Daniël Rolander) a acheté d'un Négre, appelé Quafie, le fecret qu'il avoir pour traiter les fievres les plus funeftes de Surinam, plufieurs Médecins ont parlé avantageufement du bois de quaffie, foit comme analeptique, tonique, fébrifuge, antifeptique, ou antifpamodique. Linné en a fait le fujet d'une differtation, qui eft la $22^{\mathrm{e}}$. du $6 \mathrm{e}$. vol. de fes ansinicés académiques. Spielmann en a parlé fort au long dans les intituts de matière médicale. $M$. 


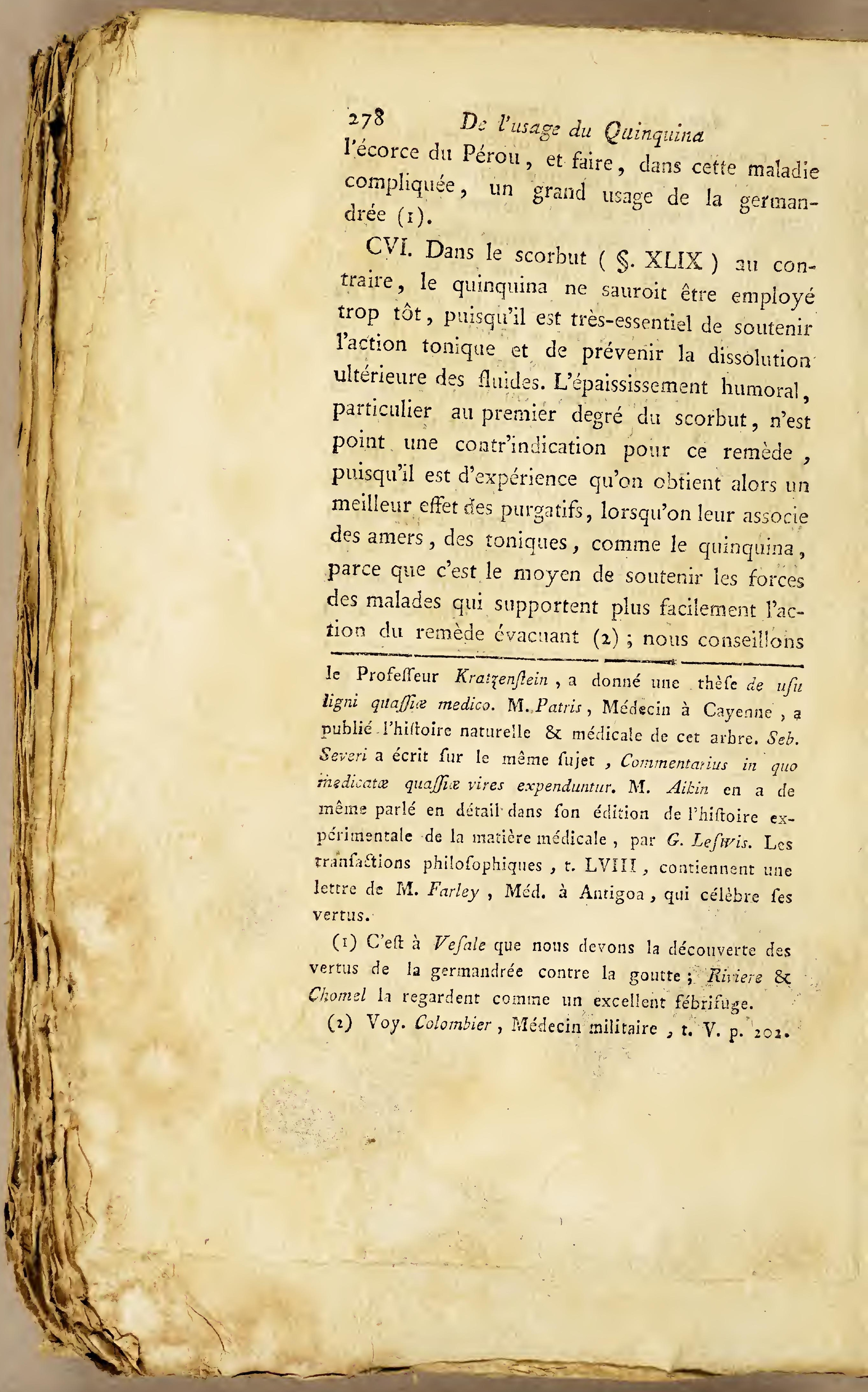




\section{dans les fievres resmittentes.}

en conséquence, de faire entrer le quiriquina dans toutes les purgations qu'on prescrit aux malades; et lorsque le temps est venu d'administrer le fébrifuge, lorsqu'a l'aide des acides enveloppés avec le sucre et des anti-scorbutiques appropriés, on a préparé son sliccès, il convient de le donier en substance combiné avec quelque sel neutre, ou bien de préférer son extrait, en qualité d’apéritif et de léger fondant. Si des accidens pressans forçent à donner cette écorce sous la forme la plus spécifique, on doit, dès que le danger est passé, recourrir à quelques doux résolutifs, parce qu’il fáut torijours craindre de supprimer la dépuration si nécessaire dans le scorbut. Et. c'est dans cetre vue qu'on fait un heureux emploi de l'oximel, uni à l'extrait de gentiane, d'absinthe ou de germandrée. En arrẹtant les excrétions oul en ne les rétablissant pas, on retient dans le corps des sucs très-acrimonieux, et dont l'action dissolvante sur les fluides et irritantes pour les solides, est trìs-considérable. N'est-ce pas faute d'avoir employé quelqques légers apéritifs, après un grand et long usage du quinquina, qu'on a observé quie le scorbut survient quelquefois d̀ la suite de cet usage dans les fièvres internittentes et rémittentes? Quoi qu'il en soit, nous remarquerons ici que, dans tous les cas de 
280 De l'usage du Quinquina

fièvre compliquée avec le scorbut, le tartre stibié est beaucoup moins avantageux que l'ipécacuanha $(\mathbf{I})$, lorsqu'il s'agit d'exciter le vomissement; qu'on doit prodiguer les amers sous la forme d'extrait, parce qu'ils ont éminemment la qualité savoneuse, dans laquelle réside une vertu tonique; enfin, quil faut, de préférence, lorsqu'on le peut, exciter les excrétions qui se font par la peau, parce que Lind s'est convaincu que la sueur étoit très-favorable aux succès du quinquina et au bien-être dés scorbutiques (2).

CVII. La cachexie scorbutique $(\mathbb{S} . \mathrm{L})$ tient de trop près, par les effets, au scorbut, pour que ce que nous venons de dire du quinquina dans ce dernier cas, ne convienne, à beaucoup d'égards, à l'autre. Ce fébrifuge paroît donc indiqué dans les fièvres qui attaquent ceuxqui vivent dans l'opulence. Cependant une opinion, fondée sur l'expérience la plus authentique et la plus répétée, porte que la crise des maladies dans les grandes villes, est la diarrhée. Mais cette crise est-elle quelquefois facile ou possible? Floyer étoit si persuadé du contraire, qu'il propose de procurer une crise artificielle par le

(1) Voy. M. Mejer, de eximio ipecacuannhe nee non aliortum quorumdam emeticorum refracta do $\sqrt{i}$ exhibitorum usu.

(2) Traité du fcorbut, t. I, p. 367. 


\section{dans les fievres rimittenies. 281}

quinquina; et le docteur Fordyce, qui croyoit pouvoir faire la médecine à Londres, comme il l'avoit faite en Ecosse, vit bientôt qu'il pouvoit et devoit faire à Londres avec de très-fortes doses de quinquina, ce qu'il faisoit en Ecosse avec des évacuations et des remèdes anti-phlogistiques. Plusieurs raisons paroissent étayer les avantages de cette pratique: $\mathrm{I}^{\circ}$. l'air des grandes villes est peu propre à soutenir l'énergie du principe vital; aussi le ton des fibres n'est jamais au degré convenable. $2^{\circ}$. Toutes les fièvres, quoique humorales, sont accompagnées dans ces villes d'un fond d'irritation, contre lequel le quinquina est très-approprié, suivant $M$. Ney (I) et qui produit mille symptômes nerveux; vrais épiphénomènes très-redoutables.

$3^{\circ}$. Mais une raison très-décisive est que la vie et le régime des opulens concilient le plus souvent à la masse des liquides une très-grande acrimonie. Ces fluides âcres sont d'une extrême dissolubilité, et il est à redouter que la fièvre prenne une forme corruptrice, que chaque exacerbation produise une fonte d'humeurs, une colliquation qui rend les effets de la maladie fort à crainare. Le quinquina prévient tous ces fâcheux accidens; il rend la fièvre plus courte,

(1) Difert. inaug. medica exhibers obfervationes medico. practicas \& chinurgicas. 
282

De l'usage du Quinquina

les crises plus rapprochées et plus complettes; et sur-tout il prévient ces longues convalescences qui toujours sont si orageuses. Plus on se hâte de l'administrer et plus les succès en sont évidens et assurés. Mais nous nous garderions bien de le proposer autrement que, combiné avec des purgatifs, tant qu'il n'y a rien d'urgent. Cette combinaison (I) est des plus précieuses; it est même impossible de pouvoir la remplacer. Non seulement le quinquina résiste alors à la pourritı. re, à la dégénération ultérieure de la masse humorale, mais encore il diminue et affoiblit les exacerbations, en même temps qu'il donne une nouvelle activité aux purgatifs, et rend l'évacuation plus décisive et plus favorable. Il paroît, d'après les propriétés purgatives et fébrifuges du cuinquina-piton (2), que cette écorce doit mé-

(I) Voy. M. Duhaume, réflexions fur le quinquina \&x fur l'ufage de cette écorce alliée aux purgatifs dans le traitement des fièvres continuës rémittentes, foit fimples, foit accompagnées d'affętions comateufes $8 x$ de paralyfies, à la page 106 de fon traité des remèdes domertiques.

(2) M. Mallet, Docteur-Régent de la faculté de Paris, a donné un Mémoire fur le quinquina de la Martinique, connu fous le nom de quinquina-piton; imprimé à la page 102 de la féance publique de la. Faculté de Médccin de Paris, du 9 décembre I770. Par l'analyfe chimique, par les faits comparés, on voit 


\section{dans les füvres rémittentes.}

riter la preference sur culle du Pérou, toutes les fois que, le danger n'étant pas pressant, il convient de combattre en même temps la fièvre et les sucs grossiers qui engouent les premières voies.

CVIII. L'humeur herpétique ( $\$$. LI ) ne présente pas de plus grande indication que celle de pousser à la peaiu, autant que peut le permettre la nature de la fièvre. La décoction de douce-amère remplit très-bien ce but, sans compter qu'elle a une action spéciale sur la cause particulière (i) des dartres. On la donnera aiguisée, si rien ne s'y oppose, avec quelques gouttes de vin d'antimoine, notamment sur le déclin des exacerbations, parce qualors la nature se prête aux évacuations cutanés. Les sinapismes ou les vésicatoires, selon l'occasion ,

que ces deux efpèces de quinquina font de même nature; mis avec cetre différence que la réfine eft furajoutée à la partie faroneufe du quinquina du Pérou, \& que, dans le quinquina-piton au contraire, s'il y exifte un peu de gomme à nu, les principes d'ailleurs y font dans un état de combinaifon plus exact, \& y forment un corps favoneux plus abondant \& plus parfait. Le quinquina-piton eft fébrifuge, cathartque \& vomitif. Voy. encore Journ. de Phy o octob. I790; p. $24^{\text {I. }}$

(I) Voy. M. Carrere, traité de la douce-amìre, \& lettre de M. Paris à M. Carrere dans la Gazette falutaire, année $1783, \mathrm{n}$. XLVIII, C. 3. 


\section{$284 \quad$ De l'usage du Quinquina}

peuvent être dans le même temps, de beaucoup d'utilité, par la vertu qu'ils ont, en excitant la peau, de réveiller les oscillations nerveuses et leur donner plus d'ordre, en les attirant vers un centre commun et les humeurs à leur suite. Nous croyons encore qu'une mixture faite avec l'extrait de douce-amère, le rob de sureau, le nitre et l'eau de chardon benit, peut être utile. En outre, pour que les autres remèdes coopèrent avec l'indication dont nous venons de parler, on ne saignera pas, autant que faire se pourra, pendant le paroxisme, mais on placera la saignée, jugée nécessaire, pendant la rémission. Les émétiques et les purgatifs ne doivent point être épargnés, et lorsque les évacuations auront été suffisantes, le quinquina sera administré, associé d'abord avec le tartre stibié, à forte dose (I), et donné ensuite en apozème avec la douce-amère, la bourrache, la chicorée.

(1) La dofe qui a paru la plus convenable, eft de douze ou quinze grains dans une pinte de décoction, \& de vingt ou vingt-quatre grains fur une once de quinquina en poudre, incorporé avec du firop, pour en faire un opiat. On fait que, par l'action du quinquina, le tartre ftibié elt décompofé, \& qu'il n'eft plus émétique. Voy. Mém. de la Société royale de Médec. de Paris, t. III. g. 242 de l'hiftoire. 


\section{dans les fièrres rémittentes: $\quad 285$}

Nous ne dirons rien sur la gale, parce qu'elle ne demande pas un traitement particulier. Quand au virus vénérien, il n'exige d'autre modification de la méthode propre à la fièvre, que de faire un grand usage du bois de Quassie, par le secours duquel M. Tissot a guéri des maladies vénériennes.

CIX. Le quinquina cause quelquefois des obstructions (I), c'est un reproche qu'on peut faire à son usage inconsidéré; et la fièvre emporte quelquefois de vieux engorgemens, c'est un éloge qu'il faut donner aux mouvemens accélérés qu'elle occasionne. Le fébrifuge est donc contrindiqué par les obstructions ( $\$$. LII) antérieures à la fièvre rémittente. Cette proposition n'est pas admissible dans sa totalité. Si la fièvre survient à des tempéramens obstrués, et que chaque exacerbation règulière et simple, semble dégager le malade, diminuer ses souffrances, fondre les embarras qu'il porte dans les viscères; pour lors la fièvre est de la plus grande utilité. Aucune indication ne porte donc à la supprimer et l'effet du spécifique administré seroit toujours fâcheux, jamais indifférent. Mais

(2) Voy. M. Buechner, de pracipuis ufus corticis pesuviani contra-indicantibus; \& M. Lentin, de corticis penuyiani cum mercurio nupti ad febres rebelles virtutibus, \&x. 


\section{De l'usage du Quinquina}

si less tempéramens obstrués, ont à souffrir d'une fière dont chaque paroxisme irrégulier et grave semble ajouter aux funestes atteintes des organes; si des obstructions squirreuses deviennent de plus en plus douloureuses et par-là menacent de s'enfiammer et d'empirer; si les accidens cachectiqlies augmentent d'une reprise à l'autre, alors le quinquina devient indiqué, parce que la fièvre offre des indications plus urgentes que les complications qui la dénaturent. Bien plus, on ne peut quelquefois se promettre la résolution des obstructions, des empâtemens, qu'en usant du quinquina, et à hăute dose. Ainsi, M. Maret (I) ayant à traiter un enfant de 9 ¿ Io ans, que plusieurs accès de fièvre tierce ou double-tierce avoient jeté dans une bouffissure universelle, avec le ventre gros, et tamáfaction de la rate, employa le quinquina pour combattre un retour de double-tierce, quoique les urines fussent rares, orangées, avec un sédiment briqueté, quioiquie les engorgemens parussent le contrindiquer d'une manière trèsformelle. M. Maret donna le fébrifuge dans l'apyrexie, à la close de demi-gros de deux en deux heures. Il le continua après la cessation de la fièvre, pendant huit jours, d'abord de

(i) Gazette falutaire A. I782, n०. XXX, col. $7^{\circ}$ 
trois en trois heures, ensuite de quatre en quatre, puis de six en six heures, puis deux fois chaque jour, enfin, une seule fois. La leucophlegmatie disparut, la rate reprit son volume naturel et l'enfant se porta à merveille. De Haen (I) a de même vu réussir très-promptement le quinquina employé sur un sujet qui, à la suite de plusieurs paroxismes de fièvre rémittente et intermittente, étoit devenu ictérique. On sait que Camérarius (2) a vanté le quinquina dans la jaunisse; que Sénac (3) a prétendu qu'on ne pouvoit guérir l'hydropisie qui survient aux fièvres, qu'avec cette écorce; enfin, que Storck (4) a avoué l'usage du quinquina pour enlever les obstructions et certaines fièvres qu'elles compliquent. Nous ajouterons ici que ce n'étoit qu'avec le quinquina, mis en digestion avec la racine de gentiane et le sel ammoniac dans de l'esprit de vin, qu'on délayoit ensuite dans une certaine quantité de bon vin, dont on faisoit prendre aux malades deux fois par jour, qu'on parvenoit à détruire la tension des hypocondres qui persistoit après la guérison,

(I) Ratio medendi, t. VI, pag. 25.

(2) Exercitatio qua corticis ufus à febre ad icterum exsenditur.

(3) De recondita febrium, \&c., pag. 392.

(4) Annus medicus, edit, de M. Aubert, t. I, p. 88. 
par le quinquina, des fièvres épidémiques de Pesaro ( $\mathrm{I}$ ). C'est d'après une suite d'observations pareilles que M. Girard a été convaincu que l'anasarque, l'ascite, la toux, le flux dyssentérique, etc. etc., n'étoient point par eux-mêmes une contr'indication à l'usage du spécifique (2).

Mais, quelqu'utile que soit le quinquina contre les obstructions qui peuvent admettre ce fébrifuge, il paroît, d'après l'expérience de plusieurs praticiens, que la Benoite (3), ce fébrifuge

(1) Voy. Lancifi, de noxiis palud. effluv. p. 206.

(2) Voyez Journal de Médecine militaire, t. VI, p. 3 I \& \& fuiv., \& t. VII, p. 488 \& fuiv.

(3) La racine de la Benoitz ou Caryophyllée, vient de nos jours, d'être mife à côté de l'écorce du Pérou, pour les vertus fébrifuge, anti-feptique \& autres. $M$. Buchhave (obfervationes circà radicis gei urbani fivè caryophyllate vires, traduites en allemand par M. Tode, \& publiées dans une (econde édition), a le premier écrit fur fes propriétés, confirmées par divers Médecins de Copenhague; MM. l'Archiatre Aaskou, le Profeffeur Callifen, les Docteurs Lang, Schoenheyden \& Tode. Plufieurs Praticiens de Friboug , MM. le Profefeur Stararafrig \& Zanner, les Docteurs Klein \& Anderwerth (difl. inaugur- medic. fiftens confitutionem anni 782 , totius $\mathcal{E}$ anni I783, ad folficium ufizum ufquè, par M. Anclerwerth), en ont retiré autant de bien que de l'écorce du Pérou. On a de M. Fiederic Anjou de Mofcow une differtation fur le même médicament (de radice caryophyllate vulga is off. Juègeo urbano Lim. ) dans laquelle; entr'autres indigêne 
dans les fièvres rémittentes. $\quad \mathbf{2 8 9}$ indigène qu'on vent stibstituer an quinquina, liii est préférable dans les fièvres avec obstruction au foie.

CX. C'est à la faveur de la distinction qu'on vient de faire sur les indications du quinquina dans le traitement des fièvres avec obstruction, que nous déterminerions l'emploi de ce fébrifuge dans celui des fières compliquées avec des ulcéres internes, si nous pouvions donner d'autres détails sans nous répéter. Nous ne nous permettrons qu'une réflexion qui est que, dans ces maladies, les vésicatoires sont presqu'indispensables pour suppléer à la suppression du pus qui, pour l'ordinaire, est l'effet de l'érétisme et de l'ardeur fébrile.

faits intéreffans, on trouve que la benoite a guéri une fièvre hémitritée, accompagnée d'accidens très-graves, qui paroiffoient conduire à la confomption; \& que, par l'analyfe chymique, une demi-once de racine de ce végétal a fourni 30 grains d'extrait réfineux' \& 20 grains d'extrait gommeux. On voit, par la théfe de $M$. le Profeffeur Webren (de Nonnullorum febrifugorum virtute \& Speciatim gei urbani radicis eficacia), que la benoite jouit d'une grande vertu contre les fièvres, fur-tout contre celles qui font accompagnées d'obttructions au foie; \& quoique des Médecins Suédois, au rapport de M. Murray (apparatus medicaminum, \&c., t. III, p. 97), aient été moins heurenx avec ce fébrifuge, on ne doit pas moins le coufidérer comme un remède efficace. 
CXI. Quels que soient les accidens que suscite la complication d'une fièvre stercorale, ce n'est jamais avec le quinquina qu'il est permis de les combattre, à moins que ce ne fùt avec le quinquina-Piton. Les évacuans en sont les vrais remèdes; et quoiqu'on ait observé que le quinquina produit un effet cathartique chez les malades dont les premières voies contiennent beaucoup de saburres, on ne doit pas se croire autorisé à administrer ici ce médicament, parce que la diarrhée n'a lieu, par l'effet du quinquina, que lorsque l'engouement des entrailles est déterminé par une véritable atonie : condition qui ne se trouve pas dans les fièvres stercorales, du moins dans leurs principes. Si l'irritation que les saburres excitent dans les premières voies, s'opposent à l'administration des émétiques modérément actifs, on doit recourir aux tisannes émollientes, animées par le tartre stibié, et seconder leurs effets par des lavemens purgatifs. Autrement, l'émétique en lavage, les purgatifs bien dosés, et dans leurs intervalles, le tartre stibié à doses réfractées, sont des secours qu'il faut employer avec d'autant plus de précipitation, que la fièvre stercorale dégénère souvent d'une manière aussi rapide que fâcheuse. Le crachement de sang, le délire, la foiblesse, les stecurs qu'on rencontre souvent dans ces 
dans les fiérres rémititentes.

maladies, ne contr'indiquent point les moyens actifs de les traiter; car c'est alors qu'on voit les symptômes péripneumoniques aggravés par la saignée, et enlevés, comme par enchantement, après l'action de l'émétique: c'est dans ces cas qu'on voit les accidens les plus alarmans, tels que les défaillances, augmentés par les cordiaux qui même alors produisent quelquefois des pétéchies, tandis qu'à l'aide des évacuans, l'angoisse et la foiblesse diminuent, les forces se relèvent les épiphénomènes disparoissent, et la fièvre marche à grands pas vers sa terminaison naturelle. On l'a vérifié dans bien des occasions et notamment dans la fièvre de Lausanne, décrite par Tissot. N'est-ce point après de telles observations, que l'auteur des pensées sur le relâchement du corps humain et sur l'abus du quinquina dans ces cas et autres (I), soutient que les évacuans sont plus utiles dans le relâchement que tout ce qui fortifie.

CXII. La fièvre vermineuse ne réclame pas un autre traitement, lorsqu'elle est de la classe des stercorales ( $\$$. LIV). Le tartre stibié (2) y réussit sur-tout comme évacuant et comme anthelmintique. Mais cela n'empêche pas qu'on

(1) Some thoughts on the relaxation of human bodie, \& $8 \mathrm{C}^{\circ}$

(2) Voy. Vogel, de ufu vomitoriorun ad ejicierados vermes. 
292. De livage du Quinquina

ne fasse usage en même-temps des vermifuges; de leau dans laqueile on a fait bouilir du mercure cru, ou d'une mixture frite avec une partie de suc de limon et deux parties d'huile, etc. lorsqu'il y a beaucoup d'érétisme; de l'helminthocorton et autres vermifuges connus, lorsqu'on n'a pas cet inconvérient à craindre. Si la propagation des vers étoit l'effet de linfluence de la fièvre, le quinquina rempliroit à la fois les deux indications. C'est sans doute par cette raison que Ramazzini (I) ne trouva pas de plus souverain vermifuge dans la fièvre épidémique de la constitution de 1689 ; exemple que nous retrouvons encore dans la plupart des épidémies décrites par Lancisi (2), M. Boucher (3) et autres. Si l'écorcel dure et ligneuse de l'amande de la pêche, possède des propriétés vermifuges analozues à celle des autres parties de cet arbre, ce remède deviendroit d'autant plus précieux que, suivant les observations de M. Burtin (4), il est un fébrifuge aussi sou-

(I) Dans le 2 e. vol. des cuvres de Sydenham p. 7 , E infrà $\emptyset$. XLIX. pag. 25 .

(2) De nox palud. effur. p. 169, $299,351,279$.

(3) Journ. de Méd. t. XIX, p. I80.

(4) Mém. fur les végétaur indigènes, fubftitués aux végétaux exetiques, \&xc; couronné à Bruxelles i 784 , pag. 32. 
a'ans ies fièvres rémittentes.

verain que celui du Pérou. La valériane peut être utile en qualité de vermifuge et d'antispasmodique; car combien n'y a-t-il pas de maladies périodiques, qui n'exigent qu'un antispasmodique approprié (I)! Nous nommerons encore le chardon benit, dont les propriétés anthelmintiques ne sont pas assez connues, et dont les qualités fébrifuges peuvent être appuyées par le témoignage de M. Tode (2). On sait que le camphre est anti-spasmodique, verminge (3), anti-septique, etc.

CXIII. Quant aux indications du quinquina, dans les fièvres compliquées de toute autre manière, elles sont plus ou moins décisives, suivant que les complications admettent ou rejettent l'usage du fébrifuge. Par exemple, la dyssenterie en réclame très-vite l'emploi, comme le prou. vent les observations de Cullen (4), Clarke (5),

(I) Voy. Ruer, de vi corticis peruviani antifpafrnodica; Bouteille, Mém. fur les vertus anci-fpafmodicrnes \& fébrifuges de la valériane, Journal de Médecine, t. XLIX, pag. 80.

(2) Difl: inaug. proftantifimam rationem illuftrandi ma. seriam medicam practicam fifiens.

(3) Voy. Prange, did: de camphor. virtute anthelinintica, 1759.

(4) Voy. Lind., Mém. fur les fièvres, pag. I98.

(s) Obferv. on the difeafes in long voyages to hot coun. tries, \&x. 


\section{De l'usage du Quinquina}

Sims (1), Darluc (2) et autres. M. Paris (3) nous apprend que, dans la peste intermittente, il faut administrer le quinquina de très-bonne heure et libéralement. Enfin, dans les justés réflexions que fait M. Grant (4) sur les maladies atrabilieuses, nous voyons que le quinquina est en général très-funeste dans toutes les fièvres rémittentes ou intermittentes qui en sont compliquées, du moins jusqu'à ce que tout l'épaississement atrabilieux soit dissipé, parce que la fievre est, dans presque tous ces cas, un instru. ment salutaire, et 'qu'il ne convient d'y appliquer le fébrifnge, que lorsque les forces sont très-épuisées, et que l'organe, par où se fait la dépuration, est foible er fatigué par la longueur de la na. ladie.

CXIV. Nous devons donc terminer ici nos discussions sur les avantages et les dangers du quinquina dans le traitement des fières rémittentes. Suivant nous, cette écorce est souverainement indiquée par la cause matérielle de ces fièvres, et ses succès sont d'autant plus grands que le caractère rémittent est sensible dans ces

(I) Obferv. fur les malad. épidem. p. I79,

(2) Journal de Méd, to XVI, p. $36 \%$

(3) Mém, fur la pefte, p. 22 \& 56 .

(4) Recherches fur les fievres, t. II, p. 307. 
maladies, et leur est véritablement affecté. Pour l'administrer avec fruit, sans doute, il faut des indications bien nettes et tranchantes. On les trouve dans l'intensité des accidens paroxistiques, dans la nécessité de soutenir les forces pour la coction, dans le besoin de ranimer la nature épuisée sur la fin du combat. Mais quelques bons effets qu'on puisse produire avec ce remède, ses principes, sa manière d'agir, font quelquefois redouter son administration (1). Cette crainte est fondée dans le début de toutes les fièvres rémittentes, parce qu'il est alors plusieurs indications préliminaires à remplir; elle ne l'est pas moins dans toutes les maladies dont la cause secondaire et pour ainsi dire dominante, n'est pas de nature à céder au quinquina; clle est enfin très-juste dans les fièvres compliquées oit l'affection qui s'y joint, répugne à l'emploi du fébrifuge. Un praticien judicieux cherche à détruire ces contrindications; et lorsque la fievre rénittente est lobjet majeur du traitement, il

(1) Voy. Reichard, de peruviani corticis in plutum febribus generum exhibendi opportunitaie. Kratzeintein de ufu corticis peruviani medico. Longobardi, difcorfo della china china. Bencia, lefficacia della china china. Marini, fpecimen inaugurale medicum de nimio \& improvido corticis peruviani ufu. Vefterveen, de contice peruviano, dif: Ranh, adverjain medica, t, I, \&c., \&x. 
met sa confance dans le quinquina, comme dans le secours qui en est le plus digne. Les circonstances font varier la forme de son administration; devenu nécessaire en substance, lorsqu'il s'agit de vaincre un ennemi puissant, ce remède, qu'il est si souvent utile et indispensable de combiner, n'est employé qu'en extrait, en décoction, en infusion, quand on veut diminuer son activité, son énergie. Telle est encore Ia règle qui dirige pour le doser. On est d'autant plus hcureux, qu'on satisfait à toutes les conditions requises pour bien administrer ce spécifique.

Mais à quoi serviroient tant de sages précautions, si, dans l'emploi du quinquina, on ne mettoit en usage qu'une écorce adultérine, ou qu'une substance à qui le temps ou d'autres accidens (I) ont presque enlevé toute propriété.

(I) Il efr d'expérience au Pérou, que le quinquina blane jouit de quelque vertu fébrifuge lorfqu'il eft récent, \& que le quinquina rouge y a beaucoup plus d'efficacité qu'en Europe; fans doute, parce que le principe actif de ces fubftances eft, ou volatil, ou fufceptible d'une décompofition plus ou moins prompte. Un quinquina furanne eft done une fubftance prefque tout-àfair inerte, \& l'on doịt conclure qu'une écorce qui a déjà fervi, ne doir plus être d'aucun usage. Cependant M. Percival (efjays médical and experimental \&x., $3^{e}$. effay) a prouvé que les principes médicamenteux du 
N'est-ce point assez que le meilleur quinquina réussisse toujours moins bien en Europe qu'il ne le fait au Pérou, sans avoir encore à lutter contre l'infidélité des falsificateurs et contre l'ignorance ou l'insousiance des pharmaciens. $\mathrm{O}$ combien ces écueils trop multipliés de nos jours, nuisent-ils à la réputation du quinquina, à la sensibilité des médecins, au salut des malades! Heureux ceux qui, conduits par un praticien habile, peuvent opposer d leurs maux la véritable écorce rouge (I) du Pérou, et qui, plutôt

quinquina penvent difficilement étre épuifés. Après 30 macérations à froid, \& 25 décoctions dans diférentes eaux, les réfidus, quoique parfaitement infipiles, ont donné, au moyen de la digeftion avec l'efprit de vin rectifié, une teinture amère \& aftringente; de l'autre côté, plufieurs digeftions dans l'ef́prit de vin, n'ont pas empêché que le réfidu, dont l'épprit n'extrairoit plus rien, ne communiquâat un degré manifefte d'aftringence à l'eau froide; car M. Percival prétend que la macération à froid, extrait la même quantité des parties réfineufes du quinquina qu'on en obtient par la coction, \& que cette fubftance communique fa vertu aufi-bien à l'eau froide qu'à l'eau chaude. (Voy. nore 2, p. Ig6.) Ce fait nous a été confirmé par un Apothicaire, chimifte éclairé, qui tire un grand parti, pour la guérifon des fièvres, du marc du quinquina, après la préparation de l'extrait. Ce marc léché \& mis en poudre enlève, felon lui, très:fûrement les fières intermittentes.

(1) On fait que le quinquina ordinaire ou du com- 


\section{De l'usage du Quinquina \\ que d'être inutilement gorgés d'un faux quin- quina toujours dangereux même lorsqu'il n'est}

merce, eft le jaune ou le noueux; mais qu'il en exifte une autre variété qui eft le quinquina rouge, dont lec vertus font fupérieures. Voy. M. SAUNDERs, obferva. tions on the fuperior efficacy of the red peruvian barck; \& RIGBY, an elfay on the ufe of the red perwivan bark in the cure of intermittent, \&c. Le quinquina rouge agit plus fpécifiquement, donné à moindire dofe, \& le principe qui le diftingue des deux autres, paroit confifter dans, une réfine noire, caffante, qu'on rencontre vers fon milieu, \& fuivant l'analyfe de M. Saunders, dans une plus grande quantité de fubrance imucilagineufe, \& de parties réfineufes. Aufii les nationaux le regardentils comme trop actif, \& lui préfèrent-ils le quinquina jaune. Cependant M. Jofeph de Juffeu qui a examiné \& fuivi les effets de cette écorce au Pérou, fe décicle en faveur du rouge; $\&$ M. Salnders prétend que les Efpagnols n'ont d'autre motif, en recommandant aux étrangers le quinquina jaune, que de garder pour eux le rouge, fans concurence. C'eft cé qu'on doit inférer du témoignage du feu Comie de Marichal qui, de retour d'Efpagne, ayant apporté en préfeni à M. Gleditjch, Profeffeur de Botanique à Berlin, une livre de ce quimquina, l'affura que cette écorce valoit mieus que toute autre efpèce, en ajoutant que c'étoit le fenl dont les Efpagnols fifent ufage. Il faut croire que les exportations de cette ếpèce ont été aurrefois affez confidérables, puifque Gleditfch lui-même, qui en avoit reçu en 1733 un échantillon d'écorce rouge du Pérou, enveloppé dans un papier, portant pour étiquète : cortex perusianus certus, approuvé par Tournefort, Baldouin \& 
dans les fièvres rémittentes.

qu'indifférent, prennent avec méthode ces fébrifuges négligés que la providence a répandu dans tous les pays (1), pour suppléer aux exotiques.

Barbie, affure qu'il n'y avoit point d'autre quinquina que le rouge chez les Aporhicaires de Poméranie, il y a environ 60 ans, \& qualors trois ou quatre dofes gúriffoient régulièrement les fièvres. M. Cothenius, prenier Médecin du Roi de Pruffe, dans un rapport des obrervations \& expériences fur l'écorce rouge du pérou, lu à l'affemblée de l'Académie des Sciences de Berlin, le 4 juillet 1783 , a remarqué que le quinquina rouge eft plus pefant que le quinquina ordinaire. Un gobelet du premier en poudre, a pefé cinq gros; \& un gobelet de l'autre n'a pefé que quatre gros, I4 grains. A l'analyfe chymique, huit onces: de quinquina rouge ont fourni 22 grains de fel alkali végétal, $\sigma$ grains de tartre vitriolé, 3 grains de terre martiale, 48 grains de terre calcaire, $\&$ \& grains de félénite. La mème quantité de quinquina ordinaire a clonné 30 grains de fel alkali végétal, 4 grains de tartre vitriolé, 4 grains de terre martiale, 37 grains de terre calcaire, 286 grains de félénite.

Nota. Pour completter l'iée que nous vondrions donner des différentes espèces du quinquina, nous renvoyons au Mémoire suivant. Memoire contenant la description 8. l'analyse de deux espèces de quinquina, naturels à lî̀e de Saint-Domingue, présenté à la Société royale des Sciences \& Arrs du Cap-français, \&rc. Par IV. lo Vavasceur, 8xc. Journal de Physique, tom. XXXVII, pag. $24 \mathrm{I}$.

(1) La lifte des fébrifuges indigènes s'eft beaucoup accrue, ou du moins leur hiftoire a écé rrès-perfection- 
née par les écrits publiés de nos jours. Indépendamment de ceux dont il a été queftion dans le cours de cet ouvrage, on a vanté les fuivans.

Io. L'ÉCORCE D'AULNE: Vogel en a indiqué les vertus fur l'autcrité du: Botanifte français Fabregou, \& $M$. Barailon en a conthaté les vertus. d'après les obfervations réitérées de ce Praticien, il paroît que certe écorce donnée à double dofe du bon quinquina, peut la remplacer fous toutes les formes \& dans tous les cas, oủ jufqu'à préfent, après bien des tentatives fur les médicamens indigènes, on a toujours "fini par s'en tenir au fébrifuge d'Amérique.

20. L'ÉCORCE DE MARONIER D'INDE: Zanichelli en indicua les propriétés, qui furent enfuite confirmées par Pontadera, Turra, Spielmann, Leidenfroft, Bucholz \& M. Sabarot de la Venière, Médecin de Nifmes. Zulatti, Médecin de Padoue, les a conteftées, mais les Auteurs des effais de matière médicale indigène (MM. Cofte \& Willemet), marchant fur les pas de Moehring \& de Peiper, ont démontré le contraire. M. de Lacroix, Médecin à la Ferté-Bernard, a profité d'une épidénie qu'il a obfervée en 1786 , pour conftater la vertu fébrifuge de cette écorce. ( $D_{0 y}$ gaz. de fanté, ann. I787, pag. 85.) Enfin, mon ami M. Cufon, que la nort a moiffonné au printemps de fon âge, au grand regret de ceux qui ont connu fon amour pour les fciences, \& le talent qu'il avoit pour les faire fervir au bonheur de l'humanité ; M. Cufion a préfenté dans un bon Mémoire, des obfervations fur les propriétés fébrifuges de l'écorce du maronier d'inde, \& fur les avantages que peut retirer de fon emploi la Médecine dans le traitement des fièvres intermittentes, 1788 . 
3․ L'B́CORCE DE SAULE BLANC: MM.-Cofle \& Willemet, Burtin, Duplanil, Spielmann \& autres, ont déterminé fes vertus fébrínges que $M$. Tode n'a point obfervées.

$4^{\circ}$. L'Corce DE Chêne: M. Cornette a lu dans la Séance publique de la Société Royale de Médecine, du 3 I août 1790, un Mémoire fur la propriété fébrifuge de cette écorce.

A ces fẻbrifuges indigènes, quelques obfervations ont joint l'écorce de putiet, celle du frêne, celle du prunier épineux, ou prunẹllier (Cofte \& Willemet); l'écorce dure \& ligneufe de l'amande de la pèche (Burtin); le lichen furfuracé, qui eft la moufe amère à feuilles d'abfinthe de Bauhin (Hagen, tentamen hiftorice lichenum, \&c.)

M. Tode a célébré les vertus de l'infufion aqueufe du chardon benit, de même que celles de la décoction de dent de lion faturée de fa racine; Bergins a vu de grands effets d'un mélange de quinquina $\&$ de fenevé, ou un feptième de noix vomique, ainfi que de la poudre de pied de veau, de tartre vitriolé \& de rhubarbe, mêlés enfemble.

Enfin, M. Joseph Mo ca (de Bononienfi, fcientiarum \& artium inftituto atque academiâ commentarii, t. VI.), a propofé l'ufage d'un firop fait avec parties égales de fucs exprimés (ou à leur défaut, des eaux diftillées, ou des infufions faturées) de fcordium, de chardon benit, de camomille \& de petite centaurée.

Le Journal de Médecine militaire, t. 2, p. 540, a expofé, dans le plus grand détail, un remède employé dans les Hôpitaux de Lille \& de Dunkerque, par ordre du Miniftre, pour la guérifon des fièvres intermittentes. 


\section{EXTRAIT des Regifires de la Société Royale de Médecine.}

MEssieurs Andry, de Chamseruet de Fourcroy, Commissaires nommés par la Société Royale de Médecine, pour lui rendre compte d'un Mémoire de M. Baumes, son Associé Regnicole, lequel Mémoire a partagé le prix proposé par cette Compagnie, sur l'usage du Quinquina dans les fièvres rémittentes, et distribué dans la Sérance publique du 30 août $\mathrm{I} 785$, en ont fait le 20 octobre, présent mois, un rapport trèsavantageux.

L'Auteur, disent ces Commissaires, a suivi strictement les termes de la question, et cependant il l'a traitée fort en grand. Rien ne paroît lui être échappé des principaux ouvrages modernes sur les fièvres rémittentes et sur toutes les épidémies auxquelles le Quinquina est applicable. Avec de tels matériaux, M. BAUMES appuye son travail sur un grand fond d'érudition, dont il ne se sert cependant que pour étendre ses vues, généraliser les résultats, et d'un petit nombre de principes clairement posés, tirer des conséquences utiles. Il "n'a pas oublié de noter beancoup de fébrifuges indigènes, 
d'après le témoignage des meilleurs observateurs, etc., etc.

D’après cet exposé, la Société Royale de Médecine a pensé que ce Mémoire, dont M. BAUMES lui a offert la Dédicace qu'elle a acceptée, étoit très-digne de son approbation, ét dêtre imprimé sous son privilége.

Ce que je certifie conforme au jugement de cette Compagnie. A Paris ce 23 octobre 1789. VICQ D'Azyr, Secrétaire perpétuel. 


\section{E R R A T A.}

N. B. T Ous les $₫$ qui sont précédés par une $\left.{ }^{*}\right)$, ont été ajoutés depuis que ce Mémoire a été couronné par la Société Royale.

Page 52, ligne if, sa, lifez la

62 , lign. 8 , genre, life zénie.

73, au commencement du $\$$, qui commence par le mot; disons, ètc., placę l'astérique $\left(^{*}\right)$.

166 , lign. I de la note (I), clini calexperiment, , lisez clinical experimentz.

I80, lign. 7, ammonical, lifer ammoniacal. 238 , lign. I I de la ${ }^{\mathrm{e}}$. note, gardaune, lisez gardanne.

242 , lign. 2 de la note 2 , après civitate; placez un point.

257 , lign. I de la note evancuantium, lisez evacuentium.

A Nismes, chez C. BE L L E, Imprimeur du Roi \& dus Département du Gard, rue des Fourbiffeurs. 1720. 


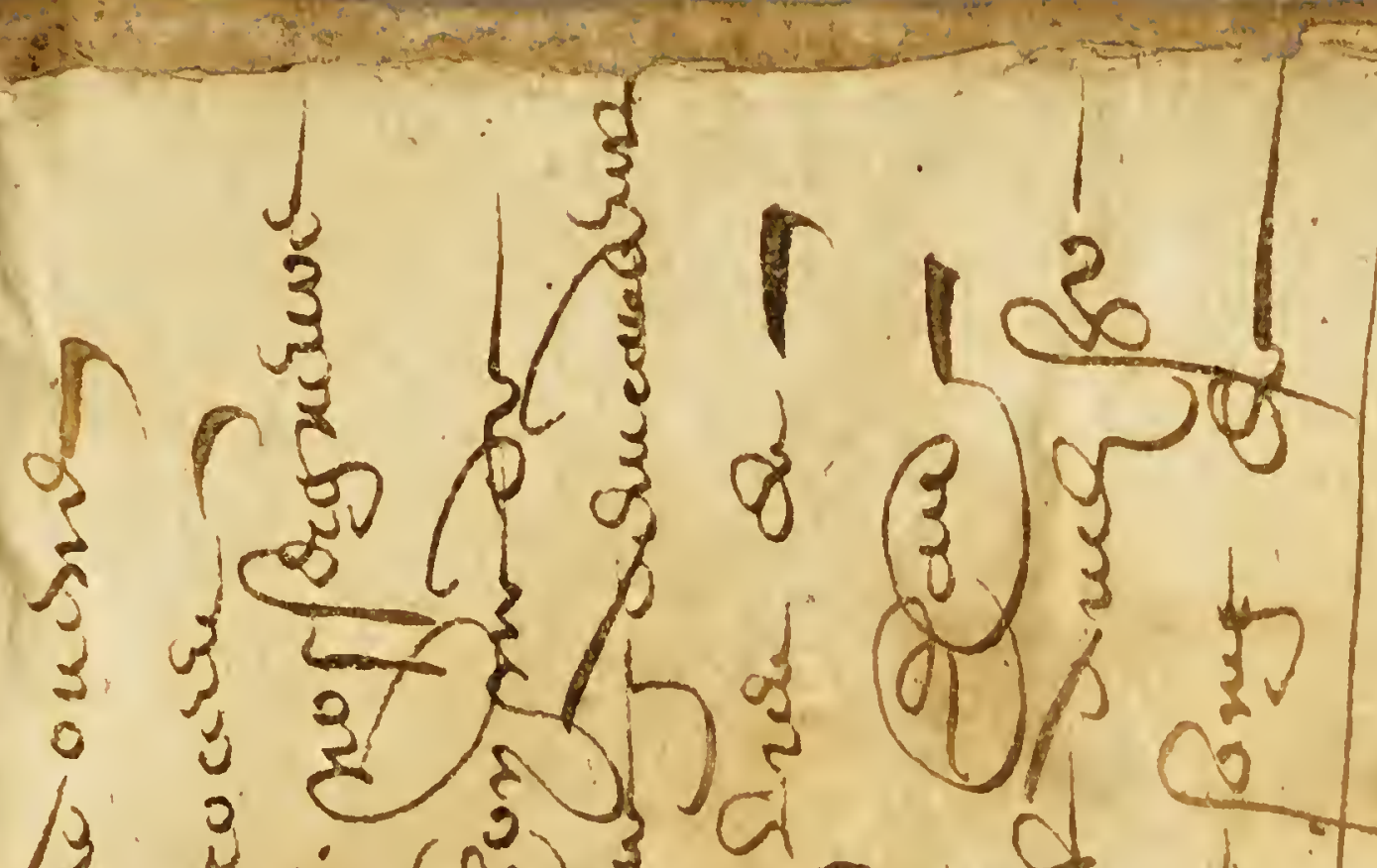

(3) क्षे Q) 3 क 3 क

of? 0 ?

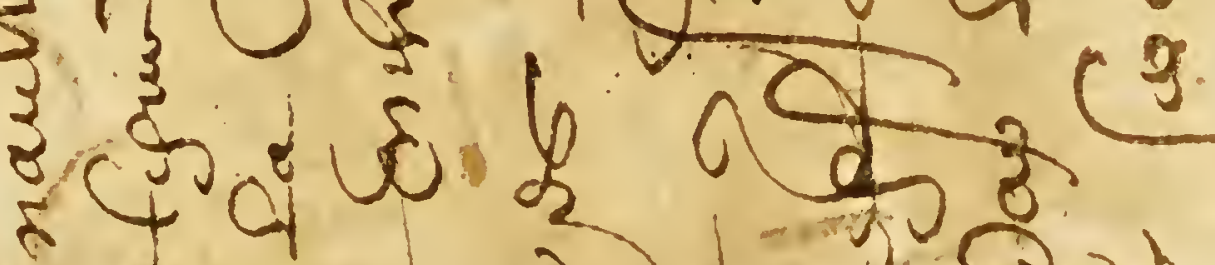

2) ปั

y. 4 a

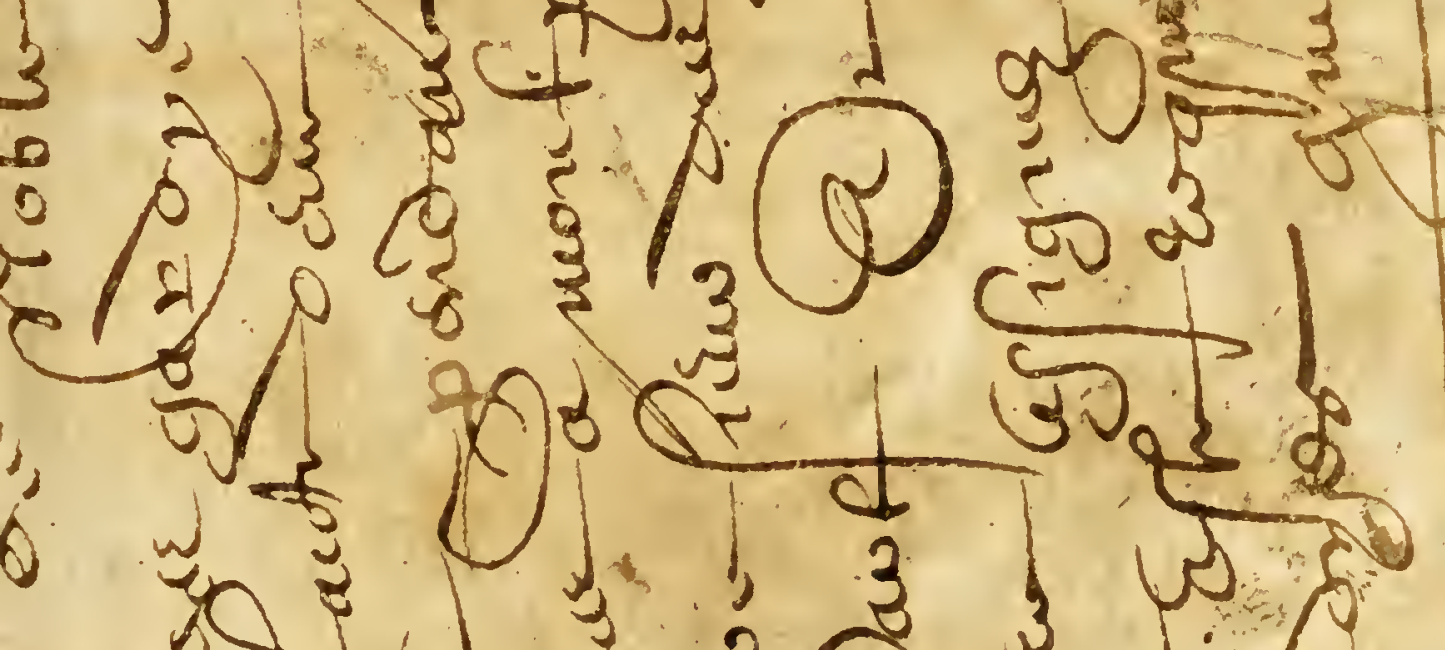

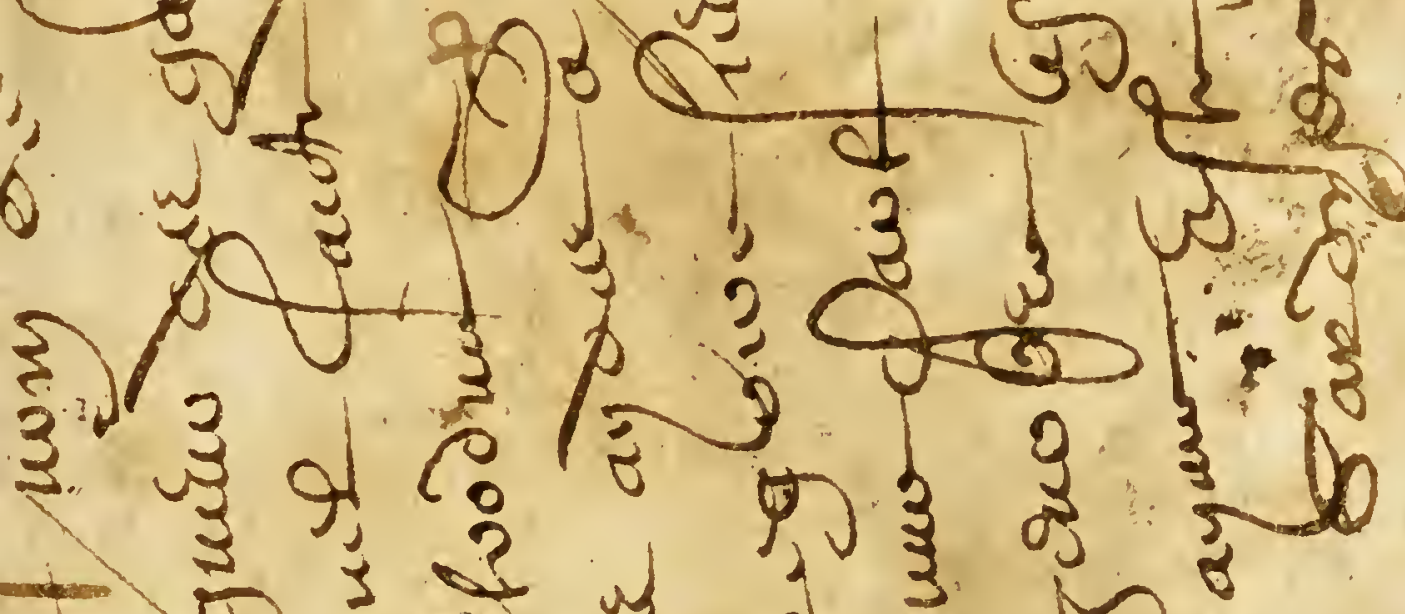

$5 y=0.00$

E. 19

B. $347 d$ 


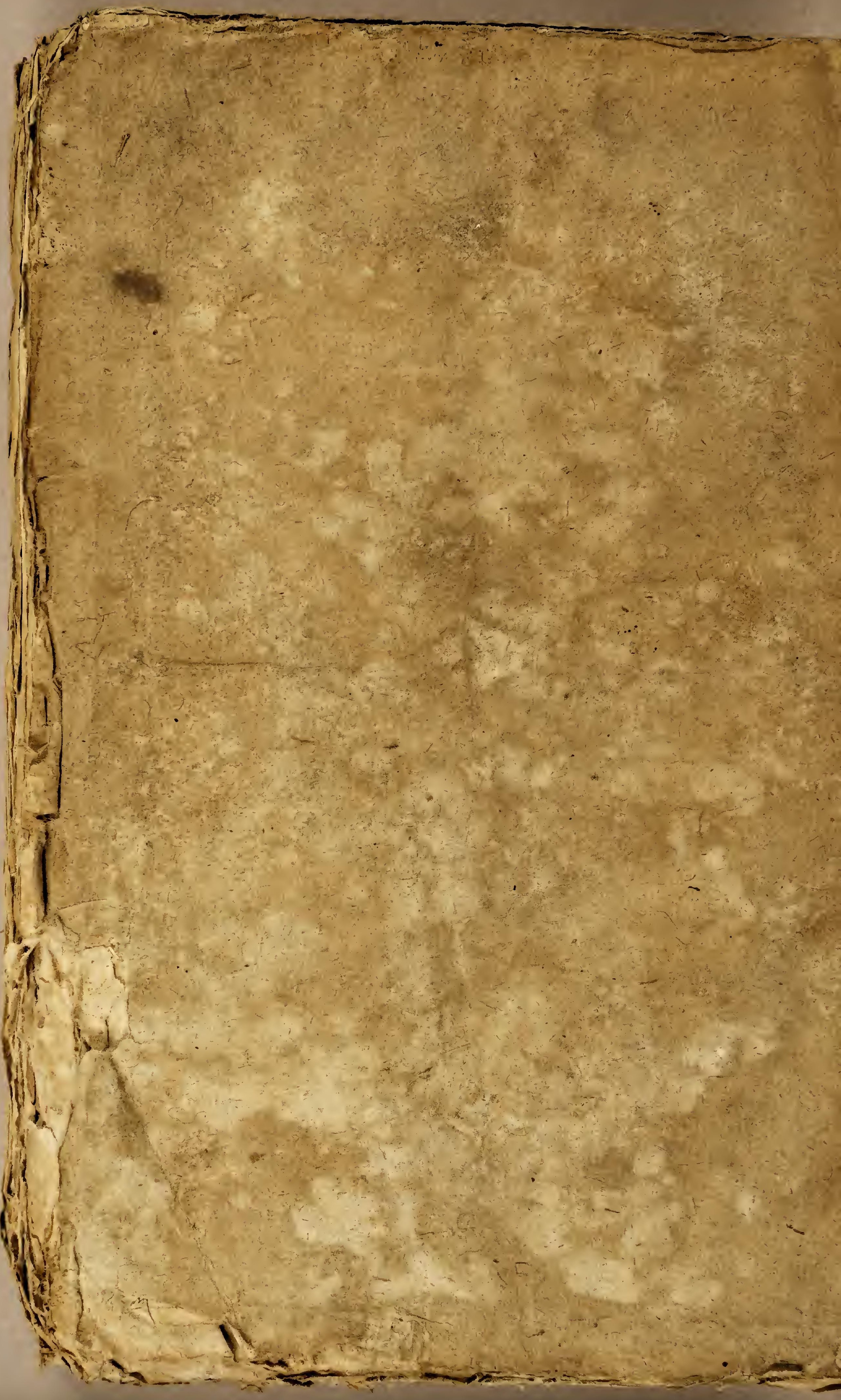

